\title{
Evasion of innate antiviral responses by picornavirus security proteins
}

Linda Jessica Visser 


\section{Evasion of innate antiviral responses by picornavirus} security proteins

\section{Het ontwijken van aangeboren antivirale responsen door picornavirus} security proteins

(met een samenvatting in het Nederlands)

Proefschrift

ter verkrijging van de graad van doctor aan de

Universiteit Utrecht

op gezag van de

rector magnificus, prof.dr. H.R.B.M. Kummeling,

ingevolge het besluit van het college voor promoties

in het openbaar te verdedigen op

woensdag 11 november 2020 des ochtends te 11.00 uur

\section{doo}

Linda Jessica Visser

geboren op 26 mei 1991

$$
\text { te Apeldoorn }
$$




\section{Promotor:}

Prof. dr. F.J.M. van Kuppeveld

\section{Copromotoren:}

Dr. M.A. Langereis

Dr. R.J. de Groot

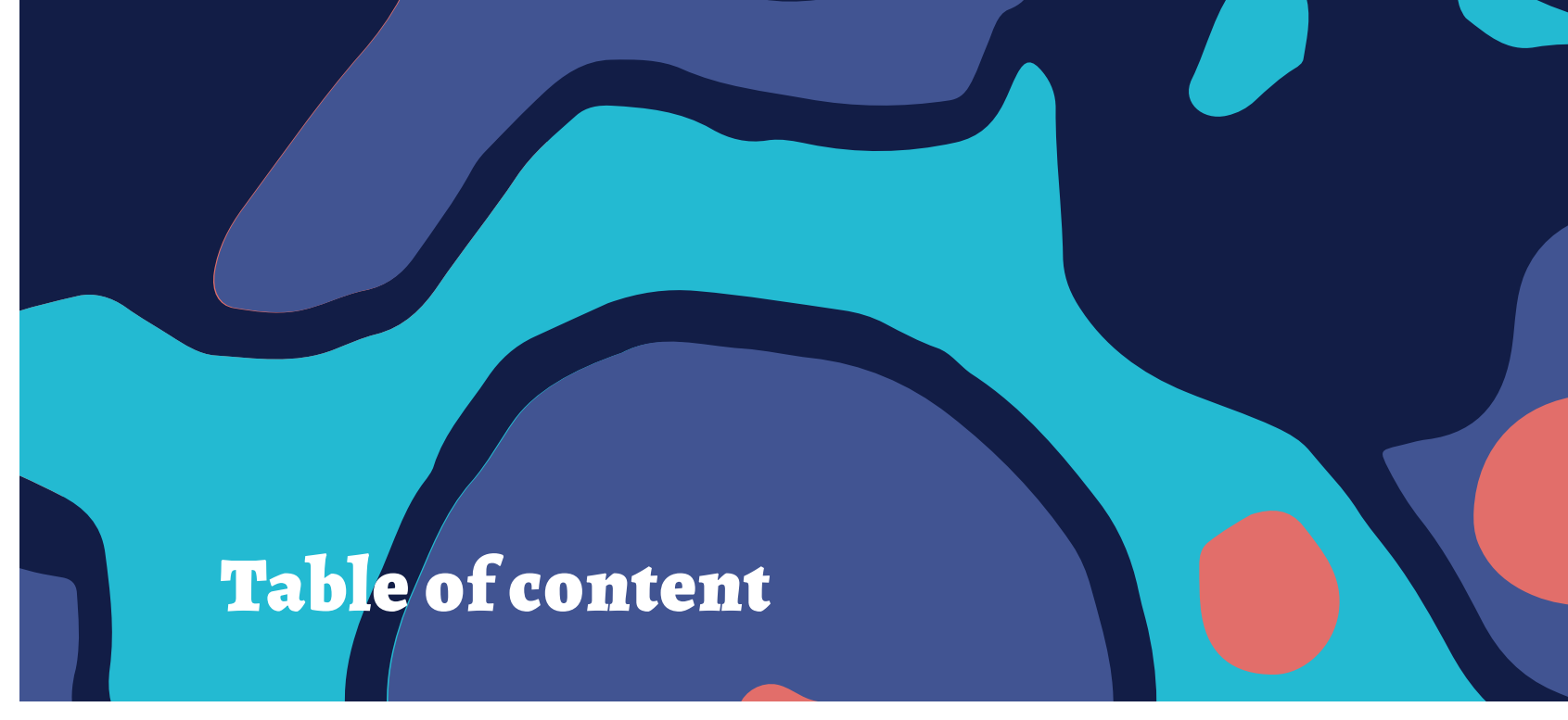

Chapter 1 General Introduction

Chapter 2 FMDV leader protease cleaves $\mathrm{G}_{3} \mathrm{BP1}$ and $\mathrm{G}_{3} \mathrm{BP} 2$ and inhibits stress granule formation

Chapter 3 Irreversible Inactivation of ISG15 by a Viral Leader Protease Enables Alternative Infection Detection Strategies

Chapter 4 Dissecting distinct proteolytic activities of FMDV L $L^{\text {pro }}$ implicates cleavage and degradation of RLR signaling proteins, not its deISGylase/DUB activity, in type I interferon suppression

Chapter 5 Essential role of enterovirus $2 \mathrm{~A}$ protease in counteracting stress granule formation and the induction of type I interferon

Chapter 6 The Aichivirus leader protein inhibits the integrated stress response by preventing $\mathrm{p}$-eIF2-mediated inhibition of eIF2B function

Chapter 7 General Discussion

Chapter 8 Addendum

Dit proefschrift werd (mede) mogelijk gemaakt met financiële steun van de Nederlandse Organisatie voor Wetenschappelijk Onderzoek (NWO) en Infection and Immunity Utrecht. 


\section{Chapter 1}

General Introduction

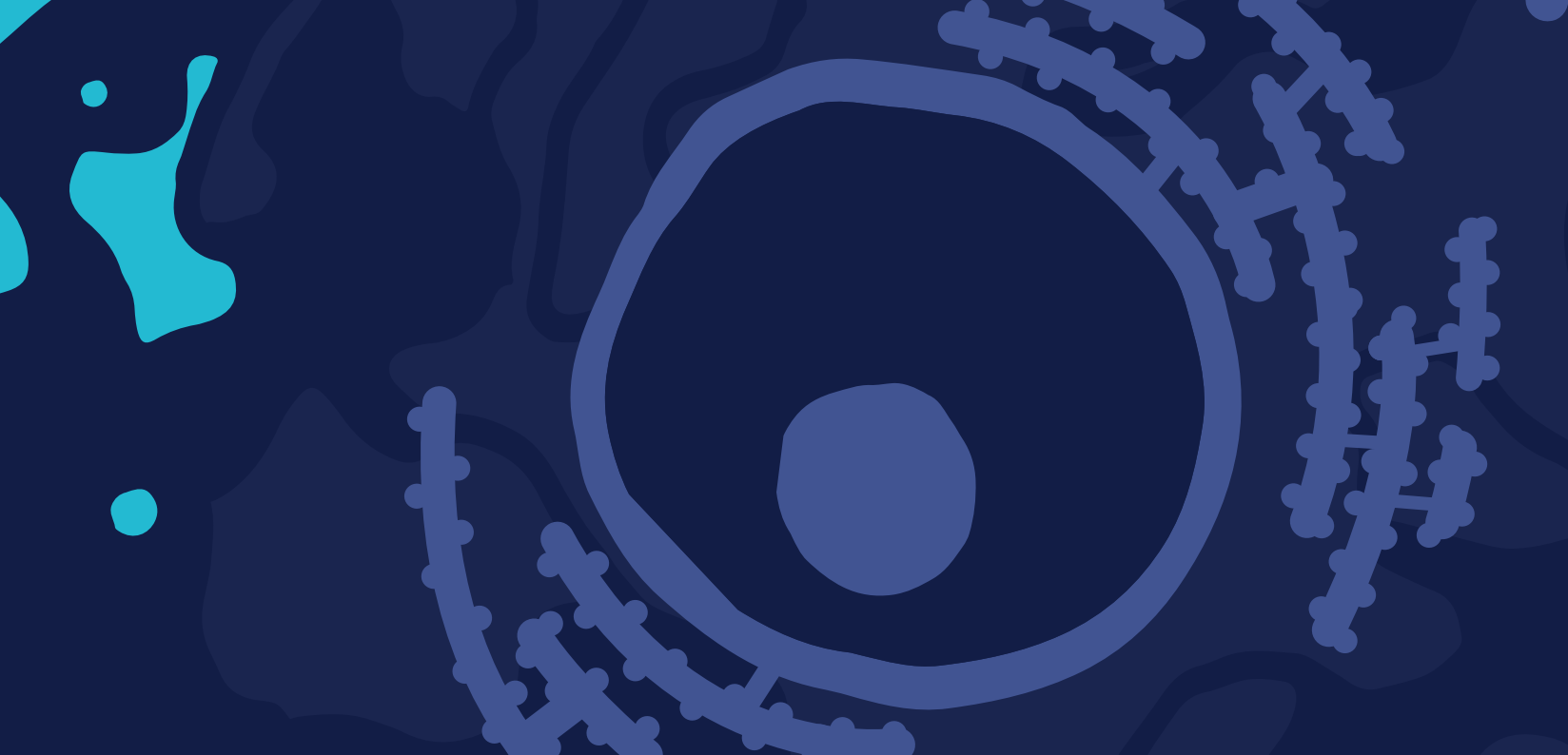


The family Picornaviridae encompasses several notorious human and animal pathogens including poliovirus (genus Enterovirus), hepatitis A virus (genus Hepatovirus) and footand-mouth disease virus (genus Aphthovirus). In 1898, foot-and-mouth disease virus was the first 'infectious agent smaller than a bacteria that could transmit disease amongst animals' that was discovered (1). Shortly after, in 1908, poliovirus was the first identified virus that transmits disease in humans (2). Although these pathogens were called viruses, the Latin term for poison, it was largely unknown what these pathogens were or how they transmitted disease. Since their discovery, these two viruses have been studied extensively and had a vital role in the development of the field of molecular virology. Understanding how these viruses translate and replicate their genome, as well as how they manipulate cellular processes, has also contributed to our current understanding of the molecular processes of the cell.

Replication of picornaviruses, like for all other viruses, can be recognized by their hosts and this triggers the activation of antiviral responses. Viruses must counteract these antiviral responses in order to spread to neighboring tissues and successfully establish infection. In this thesis, we study how selected picornaviruses evade innate antiviral responses. This chapter describes the most important picornavirus genera including their pathogenesis, the general picornavirus life cycle, antiviral responses that are relevant to the subsequent chapters, and the current knowledge of the mechanisms used by picornaviruses to evade these antiviral responses.

\section{Picornavirus classification and pathogenesis}

Picornaviruses are small non-enveloped viruses that carry a positive-strand RNA genome of $\sim 7-8 \mathrm{~kb}$, which categorizes them in Baltimore class IV. The picornavirus family traditionally consisted of 9-12 genera (3) and the viruses belonging to these genera have been studied most intensively. The discovery of novel picornaviruses in recent years has led to a rapid expansion of the family, which currently consists of 47 genera and 110 species (Fig 1)(4). Unfortunately, many of these novel picornaviruses remain poorly characterized and the majority of our understanding of picornavirus biology comes from the viruses belonging to the traditional genera. These genera include the picornaviruses that cause clinical disease, both in humans and in animals, while most newly discovered viruses appear to cause asymptomatic infection.

Most picornaviruses transmit via the fecal-oral route, while some picornaviruses can also transmit via aerosols or direct contact (4). Upon an initial round of replication at the primary site of infection, often within the digestive tract, some picornaviruses can disseminate to other organs. For example, poliovirus initially replicates in the oropharynx and/or the digestive tract, and subsequently has the potential to spreads to the central nervous system (2). Viral replication in the neurons can result in poliomyelitis, which affects $1-2 \%$ of poliovirus-infected individuals (2). Poliomyelitis is an acute flaccid paralysis disease that results in life-long disabilities and occasionally death. Here, we introduce the genera that cause clinical disease and/or that are of importance for the subsequent chapters of the thesis.

\section{Enteroviruses}

The genus Enterovirus is the largest genus amongst the family, encompassing 15 species of which 7 contain human pathogens (4). Viruses amongst the enterovirus A-D species and rhinovirus A-C species can cause disease in humans. Poliovirus, the best-known enterovirus, is a member of the enterovirus $\mathrm{C}$ species. Coxsackieviruses, echoviruses and numbered enteroviruses can induce a number of symptoms including viral meningitis, pericarditis, myocarditis and rare cases of acute flaccid paralysis (5).

Several enteroviruses have gained attention over recent years as potential health threats. One of these is EV-A71, the causative agent of hand-foot-and-mouth disease (HFMD). HFMD is characterized by fever accompanied by blisters on the hands and feet, and in the oral cavity. HFMD often affects young children. Disease progression is usually mild, but severe complications such as encephalitis, meningitis and acute flaccid paralysis can occur. Severe outbreaks of EV-A71 in Asia in recent years were associated with many cases of HFMD, neurological complications and fatalities $(6,7)$. This led to the development of an EV-A71 vaccine, which is currently only commercially available in China (8). A second enterovirus that has recently gained attention is EV-D68. This enterovirus normally replicates in the respiratory tract and induces mild symptoms. In recent years, the virus has been increasingly detected in hospitalized children and recognized to cause symptoms such as severe bronchiolitis or pneumonia (9). Large EV-D68 outbreaks in the USA in 2014, 2016 and 2018 coincided with an increase in cases of acute flaccid myelitis, a form of acute flaccid paralysis. Although EV-D68 was only sporadically detected in the cerebrospinal fluid of AFM patients, the same is true for known neurotropic viruses, including poliovirus and EV-A7I $(9,10)$. A significant increase in antibodies directed to non-polio enteroviruses, predominantly directed to EV-D68 but also EV-A71, could be identified in the CSF of the recent AFM patients (11), demonstrating the potential of these enteroviruses to induce polio-like disease. 


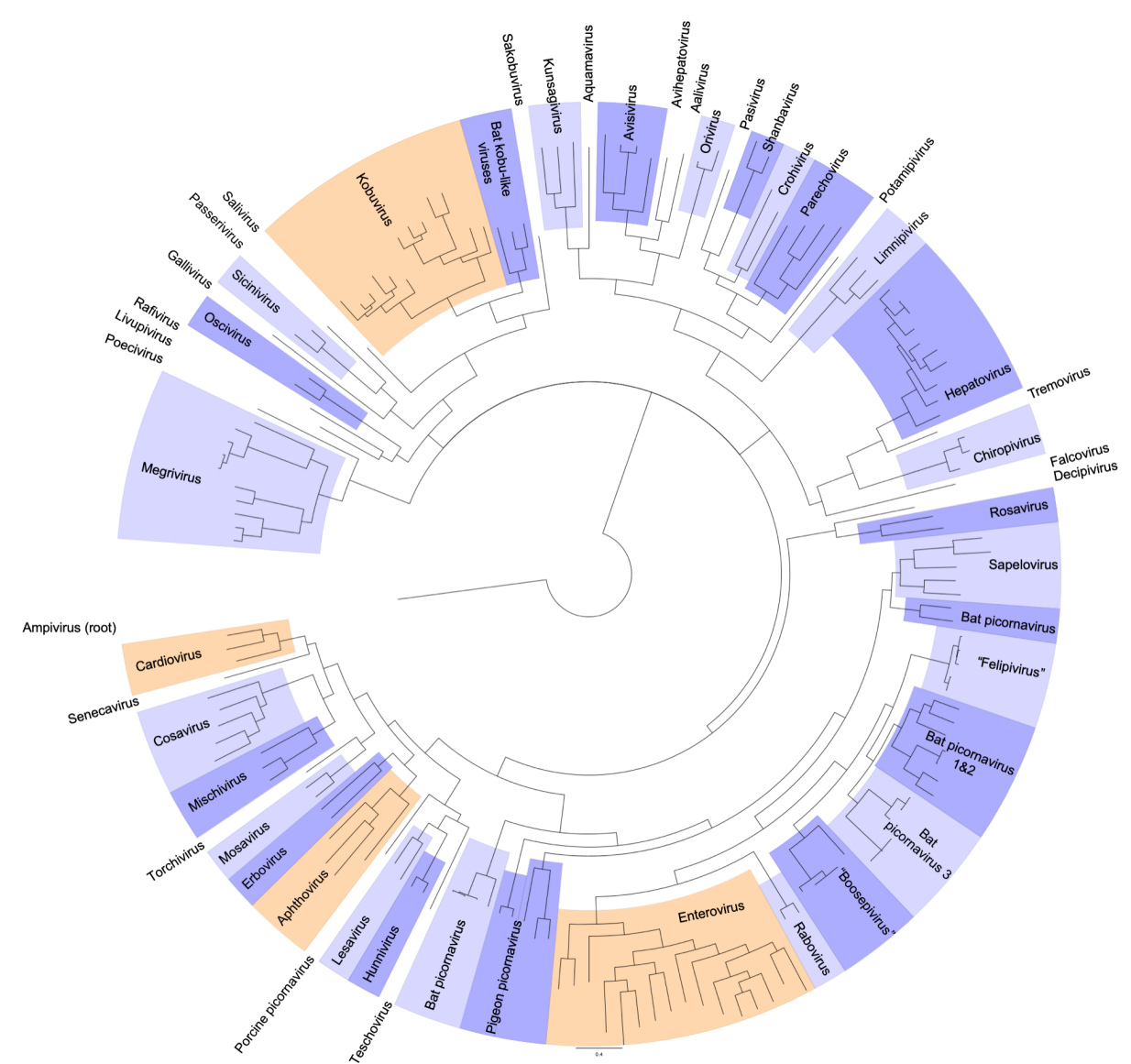

Figure 1: The picornaviridae family. P1 protein phylogenetic tree showing the relationships between the members of the family Picornaviridae as described in the 2017 ICTV report (4). Unfortunately, the freely available data file has not been updated for the 2019 ICTV report and therefore this tree only contains 35 genera. Genera studied in this thesis are highlighted in orange. Data file for the maximum likelihood tree was downloaded from the ICTV website. The original datafile was produced with MrBayes using the aamodel (blosum) and the tree was rooted to the ampivirus outgroup. This visualization was made with FigTree.

The rhinoviruses are classified as three separate species within the genus Enterovirus and replicate in the respiratory tract. Typically, they induce the common cold, the most common human viral disease with a significant socioeconomic burden due to missed working days and visits to healthcare practitioners $(12,13)$. In fact, the total socioeconomic impact of all non-influenza viral respiratory infections in the United States accumulates to $\$ 40$ billion, annually (14). Additionally, rhinovirus infections can have severe complications and contribute to exacerbations of asthma and COPD. Especially rhinovirus A and C infections can cause severe disease in young children, such as bronchiolitis and wheezing, which may require admission of pediatric patients to the intensive care unit $(12,13)$.

\section{Other human picornaviruses}

Other clinically relevant picornaviruses that infect humans can be found amongst the Parechovirus, Kobuvirus, and Hepatovirus genera and these genera will be discussed briefly.

Infection with a human parechovirus usually leads to mild respiratory or gastrointestinal symptoms, similarly to enterovirus infection. However, parechovirus infection can also lead to severe complications such as neonatal sepsis or sudden infant death (15). Particularly human parechovirus 3 has the potential to induce meningoencephalitis, which can be lifethreatening (16).

Aichivirus, the prototypic member of the kobuviruses, was first identified in 1989. People experienced gastrointestinal symptoms after consuming raw oysters, which were contaminated with an unidentified virus. The seroprevalence of aichivirus is high (80-95 $\%$ in adults), indicating that this virus is wide-spread in the human population and may indicate that subclinical infections is common (17).

Another important human pathogen is hepatitis A virus (HAV), a member of the hepatoviruses. HAV causes acute liver disease that is usually self-limiting, but may occasionally lead to complications requiring liver transplantation, and can be fatal. A vaccine for HAV is available, but unfortunately this virus remains endemic is most countries (18).

\section{Aphthoviruses}

The genus Aphthovirus exclusively contains veterinary pathogens and is best known for foot-and-mouth-disease virus (FMDV). FMDV causes blisters amongst the feet, mouth and mammary glands of cloven-hoofed animals (i.a. livestock animals). The virus is highly contagious. Therefore, outbreaks are met with extensive quarantine measures and international livestock trade restrictions. The virus has been eliminated from most developed countries, but unfortunately remains endemic in most of Africa and Asia, regions that economically depend predominantly on agriculture (19). The socioeconomic consequences of FMDV infections can be divided into direct effects (e.g. loss of animals and delay in the sales of animal products) and indirect effects (e.g. costs of vaccination and diagnostic testing). In endemic regions, these costs accumulate to \$ 6.5-21 billion, annually (20). The genus also encompasses the equine rhinitis A and B viruses (ERAV and ERBV), but these infections are mild and much less contagious. 


\section{Cardioviruses}

Cardioviruses primarily circulate amongst rodents, although they can also infect other animals. Cardioviruses can be divided into two species; the cardiovirus A species consists of encephalomyocarditis virus, while the cardiovirus B species encompasses Theiler's murine encephalitis virus, Saffold virus and Vilyuisk human encephalitis virus.

Encephalomyocarditis virus (EMCV) circulates amongst rodents worldwide. This virus can also infect captive animals such as pigs, elephants and non-human primates. In these animals the virus causes encephalitis and myocarditis, and infections have a high fatality rate. EMCV infection in humans has been reported but appears to be asymptomatic or associated with very mild symptoms (21). Another prime example of a rodent cardiovirus is Theiler's murine encephalitis virus (TMEV). TMEV infection normally presents as mild gastroenteritis, but infections of the central nervous system also occur. Experimental TMEV infection leads to chronic demyelination of the nerves and causes hind limb paralysis. The lesions are reminiscent of those found in multiple sclerosis patients, and TMEV infection is frequently used as a model system to study neuron demyelination (22).

Saffold virus (SAFV) is a human-tropic cardiovirus that was first discovered in 2007. The virus is most frequently associated with respiratory and gastrointestinal symptoms, but complications in the endocrine, cardiovascular and neurological systems as a result of SAFV infection have also been observed (23). It had been suggested that another cardiovirus also affects humans. A small isolated population of indigenous people around the Vilyui river in Russia has historically suffered from encephalitis, and this has been attributed to infection with Vilyuisk human encephalitis virus (VHEV) (24). However, the cerebrospinal fluid (CSF) of patients was injected in mice, before isolating the virus. As a result, it is unclear whether VHEV originates from man or mice. The species-specificity of one of the viral proteins $\left(L^{*}\right)$ unambiguously demonstrated that VHEV has a murine origin, suggesting that VHEV is a contaminant murine virus rather than a human cardiovirus (25).

\section{Picornavirus genome organization and life cycle}

Viruses are obligate intracellular pathogens that rely on the host metabolism to replicate. The picornavirus life cycle, from virus entry to release of new progeny, is depicted in figure 2 and described below. This section aims to describe the life cycle of all picornaviruses; however, the research on this topic has focused on only a few genera. Where dogmas are genus-specific, this will be acknowledged.
Infection starts with binding to the viral receptor to induce receptor mediated endocytosis (26). Picornaviruses use a variety of cell-surface protein as receptors, the exact receptor varies both between and within genera. Enteroviruses often use a member of the Ig-like superfamily or an integrin to enter the cell (27). Meanwhile, FMDV can bind to at least four different integrins, but can also use heparan sulfate as a receptor (28). In the endosome, the virus is uncoated to release its genome from the virion into the cytoplasm. This occurs via a virus-induced pore in the endosomal membrane.

Once in the cytoplasm, the viral genome is directly translated. Contrary to host mRNAs, the viral genome lacks a $5^{\prime}$ cap structure, which is normally used to initiate ribosome assembly. Instead, ribosomes are recruited to the viral genome via an RNA structure in the 5' UTR of the genome, known as the Internal Ribosomal Entry Site (IRES) $(29,30)$. IRES-mediated translation initiation requires some, but not all, translation initiation factors that direct normal cap-dependent translation. Which translation initiation factors are required depends on the exact IRES. Picornavirus IRESs have been sorted into 5 classes and these are distributed across the different genera (reviewed in (31)).

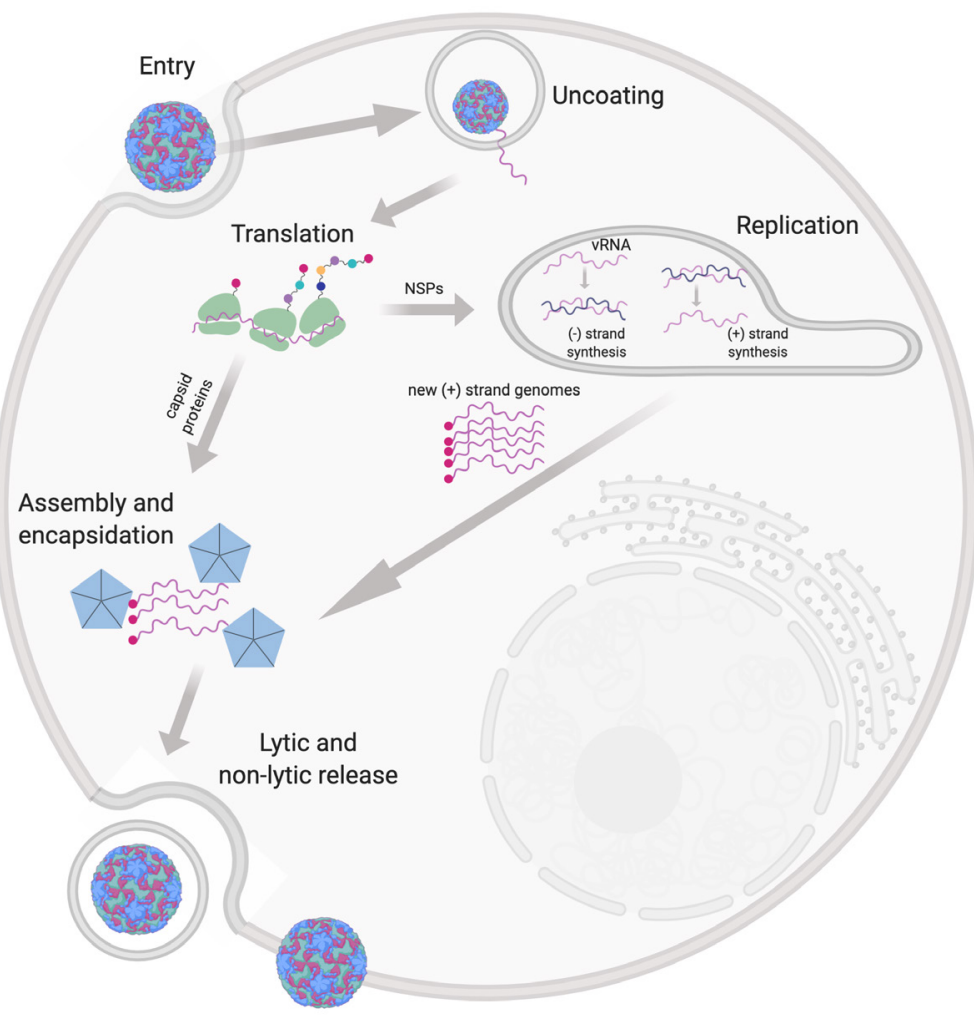

Figure 2: The picornavirus life cycle. This figure was made with BioRender 
The viral RNA is directly translated as one polyprotein that encompasses the capsid proteins ( $\mathrm{VP}_{4}, \mathrm{VP} 2, \mathrm{VP}_{3}, \mathrm{VP} 1$; collectively also known as $\mathrm{P} 1$ ) and the nonstructural proteins $(2 \mathrm{~A}-\mathrm{C}$ and $3 \mathrm{~A}-\mathrm{D})$, which are needed to replicate the viral genome. In some genera, an additional protein, the leader $(\mathrm{L})$, is encoded at the start of the polyprotein. The polyprotein is subsequently processed to 11 or 12 viral proteins and several stable intermediates (26). The majority of the polyprotein cleavages are attributed to the main viral protease, $3 \mathrm{C}^{\text {pro }}$. Some genera encode additional proteases, which contribute to the cleavage of the viral polyprotein. Notable examples are the $\mathrm{L}^{\text {pro }}$ of aphthoviruses and the $2 \mathrm{~A}^{\text {pro }}$ of enteroviruses. Aphthovirus $\mathrm{L}^{\text {pro }}$ is encoded as the first protein of the polyprotein and cleaves at its own $\mathrm{C}$-terminus to release itself from the polyprotein. Enterovirus $2 \mathrm{~A}^{\text {pro }}$ cleaves at its $\mathrm{N}$-terminus to release the nonstructural proteins from the capsid encoding region. The viral proteases also cleave several host factors to remodel the cellular landscape to benefit viral replication and/or evade the immune system. The latter will be discussed in more detail in the section "Evasion of innate antiviral responses by picornaviruses".

The first step in the replication of the viral genome is the generation of an RNA strand of negative polarity ((-)-strand synthesis) by the viral RNA-dependent RNA polymerase $\left(3 \mathrm{D}^{\mathrm{pol}}\right)$, resulting in a dsRNA replication intermediate. The negative strand subsequently serves as a template for synthesis of multiple new genomes of positive polarity $((+)$-strand synthesis). These new viral genomes can either enter another round of translation and replication, or can be encapsidated to form new virions (26). Viral genome replication takes place at virus-induced membranous structures, termed replication organelles (ROs) (26, 32). ROs are newly formed organelles that are generated using host membrane components derived from several organelles, which varies between genera (32). Most knowledge of ROs is based on enterovirus replication, although some studies on cardioviruses are available. While we aim to describe picornavirus ROs, a certain level of bias towards enterovirus and cardiovirus replication is unavoidable. Cardiovirus ROs are likely generated with host membrane components derived from the ER $(33,34)$, while enteroviruses utilize membrane components from the ER, ERGIC and the Golgi apparatus (35-39). The structure of ROs is dynamic and changes as infection progresses. Early in infection predominantly single membrane vesicles and tubules are formed, and these transform into double membrane vesicles (DMVs) as infection progresses $(34,37,40,41)$. A role for the autophagy machinery in the formation of ROs and/or the changes in RO structure has been suggested $(35,36,39$, 42). ROs are believed to support replication by scaffolding the molecules involved in RNA replication (43). It has also been suggested that the membranous structures shield the viral genome and its replication intermediate, dsRNA, from recognition by the innate immune system (44), although this was not observed in another study (45).
The newly formed capsid proteins, together with nascent viral RNA, assemble into full virions. In the case of enteroviruses, virions are assembled with VPo, the precursor of VP2 and VP4. Assembly of the virion and the interaction between VPo and the viral genome induces the processing of VPo into VP2 and VP4, to mature the virion (26). Textbooks describe picornaviruses as obligate lytic viruses, meaning that they break out of the dying cell at the end of the replication cycle. However, evidence is accumulating that new virions are released in a non-lytic manner at earlier timepoints, via fusion of virus-containing DMVs or autophagosomes with the plasma membrane $(42,46,47)$.

\section{Innate antiviral responses}

Viral infections are recognized by the host's immune system. Broadly, the immune system consists of two branches; the innate and the adaptive immune system. The well-known T-cells, B-cells, and antibodies all belong to the adaptive immune system and are essential for the eventual clearing of infections. However, raising an adaptive immune response may take several days and requires the body to first identify the pathogen. This is the role of the innate immune system. The innate immune system is trained to discriminate 'self' from 'non-self', and acts as a first line of defense. In order to identify 'non-self' molecules, the innate immune system uses an extensive repertoire of pattern recognition receptors (PRRs) to identify pathogen associated molecular patterns (PAMPs) (48). Well-known examples of PAMPs are bacterial macromolecules such as lipopolysaccharide or flagellin. The innate immune system consists of some dedicated sentinel cells (i.a. macrophages, dendritic cells and NK cells). These cells predominantly depend on Toll-like receptors (TLRs) to detect pathogens (48). However, a fundamental aspect of the innate immune system is the ability of all nucleated cells to produce type I interferons, a fundamental pro-inflammatory cytokine that is at the base of raising an innate immune response.

\section{Type I interferon}

All nucleated cells can detect intracellular PAMPs via RIG-I-like receptors (RLRs). These sensors subsequently initiate a signaling cascade to induce the production of type I interferons. The RLRs are a family of DExH/D box helicases that consists of RIG-I, MDA5 and LGP2. Both RIG-I and MDA5 have caspase activation and recruitment domains (CARD domains) that are essential for initiating the signaling cascade. LGP2 lacks a CARD domain and thereby cannot signal to downstream molecules, instead LGP2 regulates both RIG-I and MDA5 (48). MDA5 and RIG-I recognize infection by different groups of viruses. Some viruses are detected by one sensor (e.g. influenza A is recognized by RIG-I (49)), while others are detected by both sensors (e.g. measles virus)(50, 51). To explain these differences, many studies have focused on the molecular characteristics of RIG-I and MDA5 ligands. RIG-I 
recognizes RNA molecules that contain short stretches of dsRNA (at least $20 \mathrm{bp}$ ) and a 5' triphosphate group, while MDA5 recognizes long dsRNA molecules (> 1kb) $(48,52)$. The replication of the picornavirus genome generates long dsRNA replication intermediates, which have been shown to activate MDA5 (53). Nevertheless, it was recently suggested that RIG-I may also be involved in the recognition of enterovirus infection in primary cells (54).

Upon ligand recognition, the CARD domain of MDA5/RIG-I interacts with the CARD domain of mitochondrial antiviral signaling protein (MAVS, also known as IPS-1, VISA or Cardif). MAVS then activates several different TNF receptor-associated factors (TRAF proteins) to activate both the TBKI/IKK- $\varepsilon$ signaling pathway and the IKK pathway. TRAF2/3 homo- and heterodimers activate TBK1, a kinase that phosphorylates the transcription factors interferon regulatory factor 3 (IRF3) and IRF7. Phosphorylation of IRF3 and IRF7 allows these transcription factors to homodimerize and translocate to the nucleus. Simultaneously, TRAF3/6 homo- and heterodimers activate the IKK- $\alpha / \beta / \gamma$ complex, which

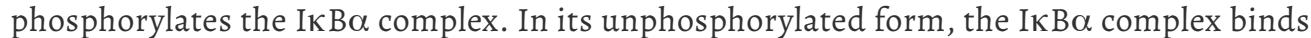
to the transcription factor NF- $\mathrm{KB}$ to inhibit its translocation to the nucleus. In the nucleus IRF3, IRF7 and NF- $\kappa B$ induce the transcription of IFN- $\alpha / \beta$ and other proinflammatory cytokines (Figure 3).

The RLR signaling cascade needs to be tightly regulated to prevent tissue damage resulting from an excessive inflammatory response. Therefore, the pathway relies heavily on post translational modifications (PTMs) of signaling molecules. Addition or removal of PTMs is a much quicker biochemical reaction than the relatively slow process of protein synthesis or degradation. Besides phosphorylation of signaling molecules (described above), the pathway is regulated by ubiquitination (reviewed in $(55,56)$ and depicted in Figure 3 ). Ubiquitination is the addition of ubiquitin (an $8 \mathrm{kDa}$ protein) to surface-exposed lysines via an iso-peptide bond. Ubiquitin itself can also be ubiquitinated, resulting in the formation of ubiquitin chains. These chains have varying $3 \mathrm{D}$ structures, depending on the lysine that is used to form the chain. Of importance for the RLR signaling cascade are K-48 and K-63 linked ubiquitin chains, which signal for protein degradation (K-48) or positively influence signal transduction $(\mathrm{K}-63)(55,56)$. The $\mathrm{K}-63$ linked ubiquitination of RIG-I is wellcharacterized. Upon RNA binding and RIG-I activation, the N-terminal CARD domain of RIG-I is first dephosphorylated, before being ubiquitinated $(57,58)$. The presence of this ubiquitin chain stabilizes its interaction with MAVS (57). Intriguingly, MDA5 appears to be regulated via phosphorylation and dephosphorylation, rather than ubiquitination (58). MAVS itself is also regulated by K-63 ubiquitination, which promotes the recruitment of IKK- $\varepsilon$ and the activation of IRF3 (59). Downstream of MAVS, ubiquitination plays an important role in both signaling arms; the signaling cascade leading to NF- $\kappa \mathrm{B}$ activation and the one leading to activation of IRF3 and IRF7. Upon interaction with upstream signaling molecules, TRAF proteins auto-ubiquitinate and this allows their respective downstream signaling complexes to bind (60-64). TBK1 is also ubiquitinated, which is essential for its enzymatic activity and thus for the activation of IRF3 and IRF7 $(65,66)$. Meanwhile, K-48 ubiquitination and subsequent proteasomal degradation of RIG-I, MDA5, MAVS, TRAF3, TRAF6, TBK1 and the transcription factors IRF3, IRF7 and NF- $\mathrm{KB}$, downregulates the inflammatory response $(67-74)$.

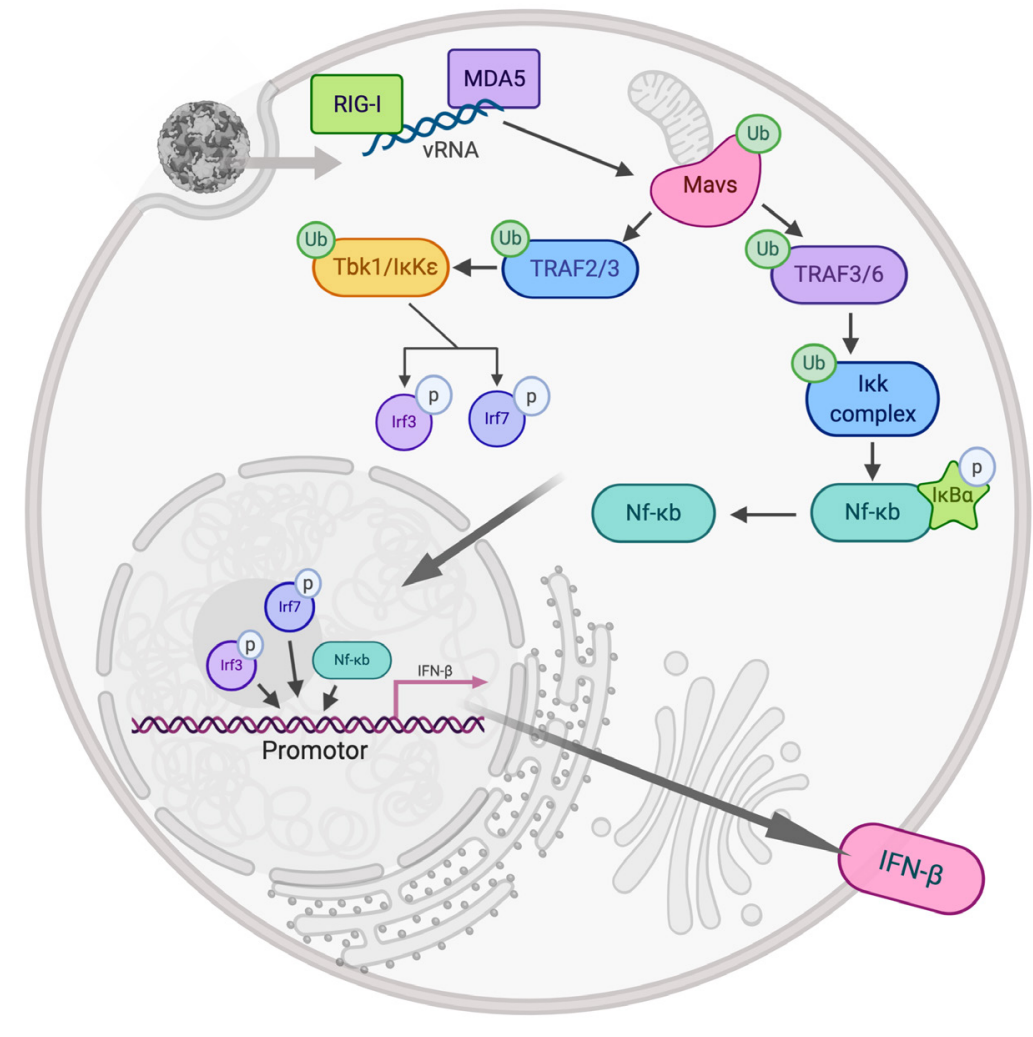

Figure 3: RLR signaling induces expression of type I interferons. This figure was made with BioRender

IFN- $\alpha / \beta$ is a cytokine that upon its secretion binds the interferon receptor (IFNAR), both on the infected cell and on uninfected neighboring cells. Upon IFN- $\alpha / \beta$ binding, Janus kinase (JAK) phosphorylates IFNAR to recruit the signal transducer and activator of transcription (STAT) proteins. The STAT proteins themselves are also phosphorylated by JAK, which allows them to dimerize. STAT dimers subsequently translocate to the nucleus to initiate expression of hundreds of interferon stimulated genes (ISGs), which have antiviral functions. Expression of the ISGs promotes an antiviral state and thereby protects uninfected cells from viral infection. There is a wide range of ISGs with many 
different activities. This group includes the signaling proteins mentioned above (i.a. PRRs, IRFs, JAK and STATs), pro-apoptotic proteins, but also proteins with an antiviral effector function (75)

Among the first discovered ISGs were the oligoadenylate synthetases (OAS) and RNAse L. OAS synthesize 2'-5' oligoadenylates upon recognition of dsRNA molecules. These oligoadenylates act as second messengers to activate the endonuclease RNAse L, before being degraded by cellular 2'-phosphodiesterases, which can break 2'-5' bonds, and phosphatases, which remove the 5' phosphate group. Upon its activation, RNAse L degrades cytosolic RNA molecules of both viral and cellular origin (75-78). Degradation of viral RNA is directly antiviral, while the degradation of cellular RNA molecules damages the host. The degradation of cellular RNA molecules promotes apoptosis, an effective host strategy when homeostasis cannot be restored and the cell succumbs to the infection.

In recent years, ISG15 has gained much attention of researchers. This ubiquitin-like molecule can be covalently linked to lysine residues in target proteins, similar to ubiquitin. The first studies into ISG15 showed it was largely protective against viral infection in mice (79-82). However, people with an ISG15-deficiency are not particularly sensitive to viral infection (83). Of note, several signal transduction proteins in the IFN signaling cascades (i.a. RIG-I, IRF3 and STATI) can be ISGylated (84-87). In addition to the ISGylation of target proteins, many other functions of ISG15 have been proposed, both intracellular and extracellular (88). However, at this time there is no comprehensive understanding of ISG15's different functions and its significance to antiviral host responses.

Another key ISG is Protein kinase R (PKR). PKR is the dsRNA binding protein that initiates the cellular stress response upon viral infection, which will be discussed in more detail below.

\section{The cellular stress response}

Cells have four sensor proteins (PKR, PERK, GCN2 and HRI) that recognize different types of cellular stress and that activate the cellular stress response. Activation of this response halts translation and gives the cell the opportunity to restore homeostasis. PKR recognizes viral dsRNA molecules (89), while PERK recognizes ER stress $(90,91)$ and GCN2 recognizes malnutrition $(92,93)$. HRI was originally described to be activated under low iron or heme conditions (94), but can also be activated by toxic heavy metal ions, and heat shock $(95,96)$.
Upon binding of viral dsRNA, PKR auto-phosphorylates leading to its dimerization and activation. Activated PKR subsequently phosphorylates the $\alpha$-subunit of translation initiation factor 2 (eIF2 $\alpha$ ) to halt translation (89) (depicted in figure 4). In an unstressed cell, eIF2 is bound to GTP and an initiator tRNA. GTP-loaded eIF2, together with translation initiation factors $1,3,5$ and the small ribosomal subunit, is known as the $43 \mathrm{~S}$ pre-initiation complex. Simultaneously, translation initiation factors $4 \mathrm{~A}, 4 \mathrm{E}$ and $4 \mathrm{G}$ (together: eIF4F) bind the cap structure of the mRNA. The $43 \mathrm{~S}$ pre-initiation complex can recognize and bind the eIF4F complex to initiate ribosome assembly, and scans the mRNA until it reaches a functional start codon. The energy required for translation initiation is generated by the hydrolysis of the GTP that is bound to eIF2. Subsequently, eIF2-GDP is released and the ribosome continues translating the mRNA. In order to start another round of translation, the eIF2-GDP complex needs to be reloaded with GTP and an initiator tRNA. To this end, eIF2-GDP binds to its guanidine exchange factor (GEF), the eIF2B complex. eIF2B ensures the release of the GDP molecule and this allows a new GTP molecule to take its place (97). The GEF is the rate limiting factor in this reaction. The high affinity interaction between phosphorylated eIF2 and eIF2B interferes with eIF2B's GEF function (98). Thereby, the binding of p-eIF2 to eIF2B prevents the GDP-to-GTP exchange of eIF2 molecules and effectively halts translation initiation (reviewed in (99)).

Halted translation results in an accumulation of untranslated mRNA molecules in the cell. RNA that is devoid of ribosomes is recognized by a group of RNA binding proteins (i.a. G3BP1, G3BP2, TIA-1 and TIAR). Accumulation of these mRNA-RNA binding protein complexes results in the formation of aggregate structures, known as stress granules (SGs) (Figure 4). Besides mRNAs and the RNA binding proteins, SGs also contain several translation initiation factors. SG formation depends both on protein-protein interaction (between the RNA binding proteins) and RNA-RNA interactions. SGs themselves were also suggested to be antiviral. Cells devoid of PKR, and thus unable to form SGs upon viral infection, produce less IFN- $\alpha / \beta$ compared to wild-type cells upon infection $(100,101)$. Additionally, numerous signaling molecules (i.e. RIG-I, MDA5 and PKR) locate to SGs (100, $102,103)$ and their localization to SGs was suggested to aid their signaling capacities (102, 104). The exact antiviral role of SGs remains to be established, to date, the most important argument for their antiviral role is the fact that many viruses actively prevent SG formation (105-111). 


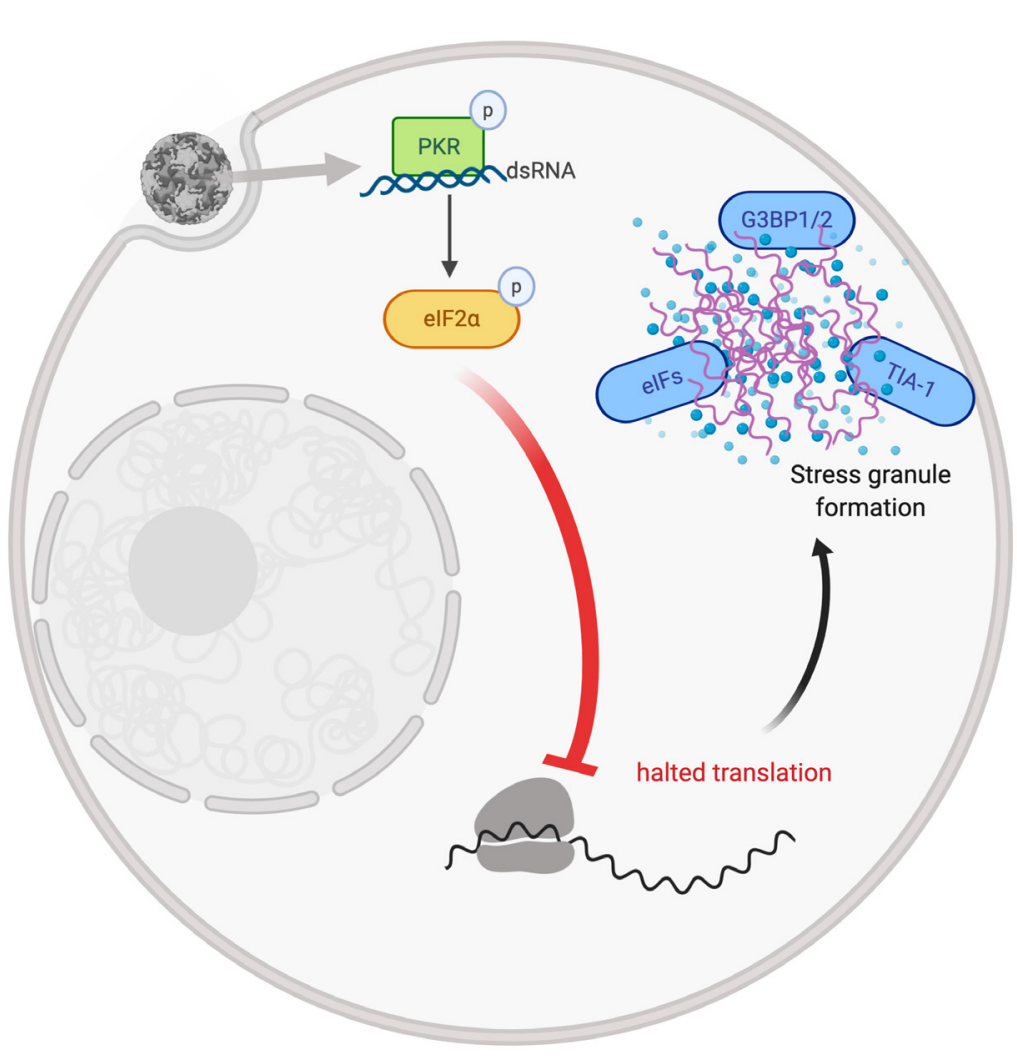

Figure 4: Activation of the cellular stress response upon viral infection. This figure was made with BioRender.

\section{Evasion of innate antiviral responses by picornaviruses}

In order to establish an infection, viruses must overcome the antiviral effects of the defense mechanisms of the host. Picornavirus $2 \mathrm{~A}$ and Leader proteins evade innate antiviral responses, and manipulate host gene expression and host translation. Because of these functions, $2 \mathrm{~A}$ and $\mathrm{L}$ are also referred to as 'security proteins' (112). The security proteins are the most diverse picornaviral proteins, inter-genera conservation is extremely low. Still, this diverse group of proteins has evolved to evade innate antiviral responses and manipulate general host metabolism. Additionally, the $3 \mathrm{C}$ protease may contribute to the evasion of host defenses, by cleavage of several host factors. Understanding the underlying molecular mechanisms not only adds to our understanding of picornavirus biology, but also illuminates unknown details of innate antiviral pathways.

\section{Inhibition of host translation}

Perhaps best studied is the effect of the different security proteins on translation of capped host mRNAs. Interfering with host translation can reduce the expression of antiviral gene products (e.g. IFN- $\alpha / \beta$ and ISGs), and thus be beneficial for the virus. Enteroviruses and FMDV are known to rapidly shut down cap dependent host translation, resulting in the predominant synthesis of viral proteins (113-115). $2 \mathrm{~A}^{\text {pro }}$ of enteroviruses and $\mathrm{L}^{\text {pro }}$ of aphthoviruses (i.a. FMDV) cleave translation initiation factor $4 \mathrm{G}$ (eIF4G) (116-118), which prevents the recruitment of pre-initiation complexes to the 5 ' cap-structure that is present on host mRNAs. The viral genome does not have a cap and thus its translation is not inhibited by eIF4G cleavage. Instead translation initiation factors are recruited to the IRES structure, present in the viral genome. The cleavage of eIF4G was shown to positively influence IRES-mediated translation (119-121). $2 \mathrm{~A}^{\text {pro }}$ of enteroviruses also targets poly(A)binding protein (PABP), which is involved in circularization of the translating mRNA, thereby enhancing translation of capped host mRNAs $(122,123)$.

Security proteins that lack proteolytic activity can also influence host translation, the prime examples are the security proteins of cardioviruses. Infection with wildtype EMCV negatively affects host translation (124). Cardiovirus 2A localizes to the nucleolus (125), interacts with ribosomes/ribosomal RNA $(126,127)$, and directs translation from capdependent translation to IRES-dependent translation (127). These collective observations suggest that $2 \mathrm{~A}$ is responsible for mediating shut-off of host translation. Cardiovirus L was also suggested to inhibit translation (128), but no mechanism has been elucidated. Cardiovirus $2 \mathrm{~A}$ and $\mathrm{L}$ interact with each other and this interaction is important for the localization and function of both $2 \mathrm{~A}$ and $\mathrm{L}$ (129). This may explain why L, indirectly, affects cap-dependent translation.

\section{Inhibition of nucleocytoplasmic trafficking and transcription}

Cardioviruses disrupt nucleocytoplasmic trafficking via their L protein (130-133). L interacts with RAN-GTPase, a protein that regulates nuclear transport by directing the activity of nuclear cargo transporters (e.g. importins) (131). Upon cardiovirus infection, specific nuclear pore proteins (nucleoporins, or NUPs) are hyper phosphorylated (133, 134). The resulting negative charge on these proteins induces the disruption of the nuclear pore complex, thereby interfering with nucleocytoplasmic trafficking and simultaneously facilitating passive diffusion. Via its interaction with RAN, L most likely localizes to the nuclear pores, where it directs a kinase to phosphorylate the NUPs. Enteroviruses also disrupt nucleocytoplasmic trafficking, and this has been attributed to $2 \mathrm{~A}^{\text {pro }} \cdot 2 \mathrm{~A}^{\text {pro }}$ of multiple enteroviruses cleaves NUPs (135-140), resulting in the collapse of the nuclear pore complex and inhibition of active nuclear transport. 
In addition to interfering with nuclear transport, picornaviruses affect host transcription. Poliovirus $3 \mathrm{C}^{\text {pro }}$ and its precursor $3 \mathrm{CD}$ can inhibit RNA polymerase I, II and III (141-143) by cleaving many different transcription factors, including well-known transcription factors such as TATA-binding protein $(142,144,145)$ and p53 (146) (reviewed in (147)). The effects of picornavirus security proteins on transcription have not been studied in great detail, but the nucleocytoplasmic trafficking disorder induced by enterovirus $2 \mathrm{~A}^{\text {pro }}$ may be required for $3 \mathrm{C}^{\text {pro }}$ to enter the nucleus, thereby allowing $3 \mathrm{C}^{\text {pro }}$ to inhibit transcription. In addition, it is noteworthy that FMDV L ${ }^{\text {pro }}$, which does not induce NCTD, accumulates in the nucleus $(148,149)$ and a number of nuclear proteins (i.e. Gemins and ADNP) have been identified as substrates and/or interaction partners of FMDV L $\mathrm{pro}^{\text {pro }}(150,151)$.

\section{Manipulation of RLR signaling and the downstream JAK-STAT pathway}

Picornaviruses also interfere with both the induction of IFN- $\alpha / \beta$ (RLR signaling) and its downstream signaling (JAK-STAT pathway). Multiple security proteins, as well as $3 \mathrm{C}^{\text {pro }}$ of several genera, have been suggested to downregulate these signaling cascades to evade the antiviral effects of IFN- $\alpha / \beta$.

During enterovirus infection, IFN- $\alpha / \beta$ gene transcription is actively suppressed. Several enteroviruses interrupt the RLR signaling cascade upstream of IRF3 phosphorylation and dimerization (152-155), although the exact underlying molecular mechanism remain to be elucidated. Multiple RLR signaling proteins are cleaved by the two enterovirus proteases, $2 \mathrm{~A}^{\text {pro }}$ and $3 \mathrm{C}^{\text {pro }}$. Upon overexpression of $3 \mathrm{C}^{\text {pro }}$, cleavage of RIG-I, MAVS, IRF7 and IRF9 was observed $(152,155-160)$, while $2 \mathrm{~A}^{\text {pro }}$ was shown to cleave MDA5 and MAVS (152, 153). However, it remains to be determined whether the cleavage events observed upon ectopic overexpression of the viral protease also occur during infection and/or contribute to the suppression of IFN- $\alpha / \beta$ gene expression. The $2 \mathrm{~A}^{\text {pro }}$ and $3 \mathrm{C}^{\text {pro }}$ of enteroviruses were also suggested to inhibits the downstream JAK-STAT signaling pathway. $2 \mathrm{~A}^{\text {pro }}$ of EV-A71 was shown to reduce the expression of the IFNAR and interfere with STATI and STAT2 phosphorylation $(161,162)$, thereby perturbing STAT activation and subsequent translocation to the nucleus. Meanwhile, $3 \mathrm{C}^{\text {pro }}$ of EV-A71 was shown to directly interfere with the translocation of STAT to the nucleus, without inhibition of STAT phosphorylation (163). Further insight into these mechanisms, their effects on ISG expression, and whether the effects are conserved amongst other enteroviruses, is needed.

FMDV also strongly suppresses the production of IFN- $\alpha / \beta$, both at the mRNA and the protein level. FMDV L $L^{\text {pro }}$ is considered the dominant FMDV protein evading the induction of IFN- $\alpha / \beta$, because a virus lacking $L^{\text {pro }}$ (leaderless virus) fails to reduce IFN- $\alpha / \beta$ levels (164). Wildtype FMDV can almost completely block the production of IFN- $\alpha / \beta$ proteins, but the reduction in IFN- $\alpha / \beta$ mRNA is only about $\sim 10$-fold. As described in detail in the section "Effects on host translation", $L^{\text {pro }}$ cleaves translation initiation factor eIF4G and this likely affects IFN- $\alpha / \beta$ protein levels. The modest reduction in IFN- $\alpha / \beta$ mRNA levels has also been attributed to the actions of $\mathrm{L}^{\text {pro }}$. $\mathrm{L}^{\text {pro }}$ degrades the NF- $\mathrm{KB}$ subunit $\mathrm{p} 65$ (148, 149). Furthermore, $L^{\text {pro }}$ was shown to decrease protein levels of IRF3 and IRF7 (165). $L^{\text {pro }}$ also possesses deubiquitinase (DUB) activity and this was suggested to suppress RLR signaling (166). The relative contribution of each of these abilities of $\mathrm{L}^{\text {pro }}$ on the suppression of RLR signaling remains to be determined. Intriguingly, the $3 \mathrm{C}^{\text {pro }}$ of FMDV was shown to cleave NEMO (also known as IKK $\gamma$ ) (167), and this could further dampen the induction of IFN- $\alpha / \beta$ mRNA. FMDV $3 \mathrm{C}^{\text {pro }}$ also prevents the translocation of activated STAT1 and STAT2 to the nucleus, thereby interfering with JAK-STAT signaling and the expression of ISGs (168).

The above may suggest that (viral) proteases are essential in circumventing RLR signaling. However, the cardioviruses depend on their leader protein, which lacks proteolytic activity, to suppress RLR signaling. Removal or mutation of the $\mathrm{L}$ coding region from the genomes of EMCV or Theiler's virus resulted in a tremendous increase in IFN- $\beta$ mRNA levels compared to infection with the respective wt viruses (169-172). Infection with wt EMCV, but not a EMCV mutant in which L's zinc-binding domain is disrupted, was shown to prevent IRF3 phosphorylation and dimerization (152, 170), but an exact molecular mechanism remains to be identified. TMEV L also affects IRF3 activation and function to suppress IFN gene expression, although subtle differences between different strains of the virus have been reported (173). TMEV also counteracts the antiviral effects of RNAse L. This virus is the only cardiovirus that expresses a $13^{\text {th }}$ viral protein, named $L^{*}$, which directly interacts with RNAse $\mathrm{L}$ to inhibit its activity (174). $\mathrm{L}^{*}$ is encoded in a second ORF that overlaps with $\mathrm{L}$ and the capsid-encoding region $(\mathrm{P} 1)$.

\section{Evasion of the cellular stress response}

PKR activates the stress response upon detection of viral RNA, resulting in the phosphorylation of eIF2 and a halt in protein translation. The halted translation subsequently results in the formation of stress granules (SGs). Viruses can evade this antiviral response in two ways; either they interfere with the signaling cascade - thereby circumventing the negative effects on translation as well as SG formation - or they exclusively interfere with SG assembly.

Enterovirus $2 \mathrm{~A}^{\text {pro }}$ cleaves translation initiation factor eIF4G. The inhibitory effect of eIF4G cleavage on host translation should theoretically trigger SG formation. Yet, poliovirus infection does not trigger massive SG formation (175), suggesting the virus counteracts this antiviral response. While IRES-translation requires eIF2 in vitro or during the early stages of infection, it becomes independent of eIF2 during the later stages of infection, 
via a process that requires $2 \mathrm{~A}^{\text {pro }}(176)$. Effectively, this ability makes the virus resistant to the negative effects of eIF2 $\alpha$ phosphorylation and non-surprisingly poliovirus infection does not inhibit the phosphorylation of PKR and eIF2 $\alpha$ (177). Despite the phosphorylation of eIF2 $\alpha$, there are hardly any SGs observed in poliovirus infected cells (175), suggesting that the virus suppress SG formation. Several enterovirus $3 \mathrm{C}^{\text {pro's }}$ were shown to cleave the RNA-binding protein $\mathrm{G}_{3} \mathrm{BPI}$, and this was suggested to negatively affect SG formation (175, 178, 179).

Cardiovirus infection does not trigger SG formation, but SGs are formed when cells were infected with cardioviruses carrying mutations in leader (103, 180). Furthermore, both EMCV and TMEV leader inhibit the phosphorylation of PKR (181, 182), suggesting that cardioviruses targets the PKR signaling pathway to prevent stress response activation.

FMDV $L^{\text {pro }}$, like enterovirus $2 \mathrm{~A}^{\text {pro }}$, stimulates eIF2-independent IRES-driven translation at later timepoints of infection (121), suggesting that FMDV infection is resistant to the antiviral effects of eIF2 $\alpha$ phosphorylation. While it was shown that infection with FMDV affects SG formation (183), no mechanistic insights have been provided. Exactly how FMDV suppresses the stress response remains to be elucidated.

\section{Aim and outline of this thesis}

This thesis aims to increase our understanding of how different picornaviruses evade the induction of type I interferons and the antiviral effects of the stress response. Chapters 2-4 focus on FMDV L pro and its ability to suppress the antiviral responses. Working with FMDV requires extensive biosafety measures. To circumvent this, we inserted FMDV $\mathrm{L}^{\text {pro }}$ in a recombinant encephalomyocarditis virus (EMCV) to create a chimeric virus, EMCV-L pro. We utilized similar chimeric EMCVs to study the evasive functions of enterovirus $2 \mathrm{~A}^{\text {pro }}$ and $3 \mathrm{C}^{\text {pro }}$ in chapter 5 , and the aichivirus L protein (genus Kobuvirus) in chapter 6.

In chapter 2 we explored whether and how FMDV evades the stress response. We demonstrate that this virus suppresses SG formation via the activity of $\mathrm{L}^{\text {pro. }}$. In chapter 3 we uncovered that FMDV L ${ }^{\text {pro }}$ has a 1000-fold higher affinity for ISG15 than for ubiquitin. Based on overexpression experiments, $\mathrm{L}^{\text {pros }} \mathrm{s}$ deubiquitinase (DUB) activity was suggested to play a role in the suppression of RLR signaling (166). However, our data suggest that $\mathrm{L}^{\text {pro }}$ has only very modest DUB activity. This raised the question whether $\mathrm{L}^{\text {pro's }}$ deISGylase activity is involved in suppression of RLR signaling. We address this question in chapter 4 , where we separated the proteolytic activity of $\mathrm{L}^{\text {pro }}$ towards RLR signaling proteins from its deISGylase/DUB activity via the introduction of specific mutations in $\mathrm{L}^{\text {pro }}$. This allowed us to investigate which proteolytic activity is needed to reduce the induction of IFN- $\alpha / \beta$ mRNA.

Chapter 5 provides a detailed comparative analysis of the ability of $2 \mathrm{~A}^{\text {pro }}$ and $3 \mathrm{C}^{\text {pro }}$ of human enteroviruses (i.e. those belonging to species A-D) to suppress both the stress response and the induction of IFN- $\alpha / \beta$. We demonstrate that $2 \mathrm{~A}^{\text {pro }}$, not $3 \mathrm{C}^{\text {pro }}$, is responsible for the suppression of both innate antiviral responses.

In chapter 6 we investigate whether and how kobuviruses suppress the stress response. We identify a novel interaction between aichivirus leader and translation initiation factor $2 \mathrm{~B}$ (eIF2B), and describe how this interaction allows $\mathrm{L}$ to circumvent the antiviral effects of the cellular stress response.

Chapter 7 provides a brief summary of the findings, a general discussion, and directions for future research. 


\section{References}

Rott R, Siddell S. 1998. One hundred years of animal virology. J Gen Virol 79:2871-2874.

2. Racaniello VR. 2006. One hundred years of poliovirus pathogenesis. Virology 344:9-16.

3. Knowles NJ, Hovi T, Hyypiä T, King AMQ Lindberg AM, Pallansch MA, Palmenberg AC, Simmonds P, Skern T, Stanway G, Yamashita T, Zell R. 2012. Picornaviridae, p. 855-880. In Ninth Report of the International Committee on Taxonomy of Viruses. Elsevier.

4. Zell R, Delwart E, Gorbalenya AE, Hovi T, King AMQ, Knowles NJ, Lindberg AM, Pallansch MA, Palmenberg AC, Reuter G, Simmonds P, Skern T, Stanway G, Yamashita T, Consortium IR. 2017. ICTV Virus Taxonomy Profile: Picornaviridae. J Gen Virol 98:2421-2422.

5. Tapparel C, Siegrist F, Petty TJ, Kaiser L. 2013. Picornavirus and enterovirus diversity with associated human diseases. Infect Genet Evol 14:282-293.

6. Xing W, Liao Q, Viboud C, Zhang J, Sun J, Wu JT, Chang Z, Liu F, Fang VJ, Zheng Y, Cowling BJ, Varma JK, Farrar JJ, Leung GM, Yu H. 2014. Hand, foot, and mouth disease in China, 2008-12: an epidemiological study. Lancet Infect Dis 14:308-318.

7. Huang J, Liao Q, Ooi MH, Cowling BJ, Chang Z, Wu P, Liu F, Li Y, Luo L, Yu S, Yu H, Wei S. 2018. Epidemiology of Recurrent Hand, Foot and Mouth Disease, China, 2008-2015. Emerg Infect Dis 24.

8. Chong P, Liu C-C, Chow Y-H, Chou A-H, Klein M. 2015. Review of Enterovirus 71 Vaccines. Clin Infect Dis 60:797-803.

9. Holm-Hansen CC, Midgley SE, Fischer TK. 2016. Global emergence of enterovirus D68: a systematic review. Lancet Infect Dis 16:e64-e75.

10. Stelzer-Braid S, Rawlinson W. 2018. Outbreaks of acute flaccid myelitis in the US. BMJ 363:k5246.

11. Schubert RD, Hawes IA, Ramachandran PS, Ramesh A, Crawford ED, Pak JE, Wu W, Cheung CK, O’Donovan BD, Tato CM, Lyden A, Tan M, Sit R, Sowa GA, Sample HA, Zorn KC, Banerji D, Khan LM, Bove R, Hauser SL, Gelfand AA, Johnson-Kerner BL, Nash K, Krishnamoorthy KS, Chitnis T, Ding JZ, McMillan HJ, Chiu CY, Briggs B, Glaser CA, Yen C, Chu V, Wadford DA, Dominguez SR, Ng TFF, Marine RL, Lopez AS, Nix WA, Soldatos A, Gorman MP, Benson L, Messacar K, Konopka-Anstadt JL, Oberste MS, DeRisi JL, Wilson MR. 2019. Pan-viral serology implicates enteroviruses in acute flaccid myelitis. Nat Med 25:1748-1752.

12. Gern JE. 2010. The ABCs of rhinoviruses, wheezing, and asthma. J Virol 84:7418-26.

13. To KKW, Yip CCY, Yuen K-Y. 2017. Rhinovirus - From bench to bedside. J Formos Med Assoc 116:496-504.

14. Fendrick AM, Monto AS, Nightengale B, Sarnes M. 2003. The Economic Burden of Non-Influenza-Related Viral Respiratory Tract Infection in the United States. Arch Intern Med 163:487.

15. Wildenbeest JG, Harvala H, Pajkrt D, Wolthers KC. 2010. The need for treatment against human parechoviruses: how, why and when? Expert Rev Anti Infect Ther 8:1417-1429.

16. Aizawa $Y$, Izumita R, Saitoh A. 2017. Human parechovirus type 3 infection: An emerging infection in neonates and young infants. J Infect Chemother 23:419-426.

17. Reuter G, Boros Á, Pankovics P. 2011. Kobuviruses - a comprehensive review. Rev Med Virol 21:32-41.

18. Lemon SM, Ott JJ, Van Damme P, Shouval D. 2017. Type A viral hepatitis: A summary and update on the molecular virology, epidemiology, pathogenesis and prevention. J Hepatol.

19. Jamal SM, Belsham GJ. 2013. Foot-and-mouth disease: past, present and future. Vet Res 44:116.

20. Knight-Jones TJD, Rushton J. 2013. The economic impacts of foot and mouth disease - what are they, how big are they and where do they occur? Prev Vet Med 112:161-73.

21. Carocci M, Bakkali-Kassimi L. 2012. The encephalomyocarditis virus. Virulence 3:351-367.

22. Gerhauser I, Hansmann F, Ciurkiewicz M, Löscher W, Beineke A. 2019. Facets of Theiler's Murine Encephalomyelitis Virus-Induced Diseases: An Update. Int J Mol Sci 20:448.

23. Tan SZK, Tan MZY, Prabakaran M. 2017. Saffold virus, an emerging human cardiovirus. Rev Med Virol 27:e1908.
24. Lipton HL. 2008. Human Vilyuisk encephalitis. Rev Med Virol 18:347-352.

25. Drappier M, Opperdoes FR, Michiels T. 2017. Nonstructural Protein L* Species Specificity Supports a Mouse Origin for Vilyuisk Human Encephalitis Virus. J Virol 91.

26. Baggen J, Thibaut HJ, Strating JRPM, Van Kuppeveld FJM. 2018. The life cycle of non-polio enteroviruses and how to target it. Nat Rev Microbiol.

27. Wells AI, Coyne CB. 2019. Enteroviruses: A Gut-Wrenching Game of Entry, Detection, and Evasion. Viruses 11:460.

28. Wang G, Wang Y, Shang Y, Zhang Z, Liu X. 2015. How foot-and-mouth disease virus receptor mediates footand-mouth disease virus infection. Virol J 12:9.

29. Pelletier J, Sonenberg N. 1988. Internal initiation of translation of eukaryotic mRNA directed by a sequence derived from poliovirus RNA. Nature.

30. Jang SK, Kräusslich HG, Nicklin MJ, Duke GM, Palmenberg AC, Wimmer E. 1988. A segment of the nontranslated region of encephalomyocarditis virus RNA directs internal entry of ribosomes during in vitro translation. J Virol.

31. Lozano G, Martínez-Salas E. 2015. Structural insights into viral IRES-dependent translation mechanisms. Curr Opin Virol 12:113-120.

32. van der Schaar HM, Dorobantu CM, Albulescu L, Strating JRPM, van Kuppeveld FJM. 2016. Fat(al) attraction Picornaviruses Usurp Lipid Transfer at Membrane Contact Sites to Create Replication Organelles. Trend Microbiol 24:535-546.

33. Dorobantu CM, Albulescu L, Harak C, Feng $Q$, van Kampen M, Strating JRPM, Gorbalenya AE, Lohmann V, van der Schaar HM, van Kuppeveld FJM. 2015. Modulation of the Host Lipid Landscape to Promote RNA Virus Replication: The Picornavirus Encephalomyocarditis Virus Converges on the Pathway Used by Hepatitis C Virus. PLOS Pathog 11:e1005185.

34. Melia CE, van der Schaar HM, de Jong AWM, Lyoo HR, Snijder EJ, Koster AJ, van Kuppeveld FJM, Bárcena M. 2018. The Origin, Dynamic Morphology, and PI4P-Independent Formation of Encephalomyocarditis Viru Replication Organelles. MBio 9 .

35. Schlegel A, Giddings TH, Ladinsky MS, Kirkegaard K. 1996. Cellular origin and ultrastructure of membranes induced during poliovirus infection. J Virol 70:6576-88.

36. Hsu N-Y, Ilnytska O, Belov G, Santiana M, Chen Y-H, Takvorian PM, Pau C, van der Schaar H, Kaushik-Basu N, Balla T, Cameron CE, Ehrenfeld E, van Kuppeveld FJM, Altan-Bonnet N. 2010. Viral Reorganization of the Secretory Pathway Generates Distinct Organelles for RNA Replication. Cell 141:799-811.

37. Limpens RWAL, van der Schaar HM, Kumar D, Koster AJ, Snijder EJ, van Kuppeveld FJM, Bárcena M. 2011. The transformation of enterovirus replication structures: a three-dimensional study of single- and doublemembrane compartments. MBio 2

38. Melia CE, Peddie CJ, de Jong AWM, Snijder EJ, Collinson LM, Koster AJ, van der Schaar HM, van Kuppeveld FJM, Bárcena M. 2019. Origins of Enterovirus Replication Organelles Established by Whole-Cell Electron Microscopy. MBio 10.

39. Suhy DA, Giddings TH, Kirkegaard K. 2000. Remodeling the Endoplasmic Reticulum by Poliovirus Infection and by Individual Viral Proteins: an Autophagy-Like Origin for Virus-Induced Vesicles. J Virol 74:8953-8965.

40. Belov GA, Nair V, Hansen BT, Hoyt FH, Fischer ER, Ehrenfeld E. 2012. Complex dynamic development of poliovirus membranous replication complexes. J Virol 86:302-12.

41. Oh HS, Banerjee S, Aponte-Diaz D, Sharma SD, Aligo J, Lodeiro MF, Ning G, Sharma R, Arnold JJ, Cameron CE. 2018. Multiple poliovirus-induced organelles suggested by comparison of spatiotemporal dynamics of membranous structures and phosphoinositides. PLoS Pathog 14:1007036.

42. Jackson WT, Giddings TH, Taylor MP, Mulinyawe S, Rabinovitch M, Kopito RR, Kirkegaard K. 2005. Subversion of Cellular Autophagosomal Machinery by RNA Viruses. PLoS Biol 3:e156.

43. Paul D, Bartenschlager R. 2013. Architecture and biogenesis of plus-strand RNA virus replication factories. World J Virol 2:32-48. 
44. Viktorova EG, Nchoutmboube JA, Ford-Siltz LA, Iverson E, Belov GA. 2018. Phospholipid synthesis fueled by lipid droplets drives the structural development of poliovirus replication organelles. PLOS Pathog 14:e1007280.

45. Melia CE, van der Schaar HM, Lyoo H, Limpens RWAL, Feng $Q$, Wahedi M, Overheul GJ, van Rij RP, Snijder EJ, Koster AJ, Bárcena M, van Kuppeveld FJM. 2017. Escaping Host Factor PI4KB Inhibition: Enterovirus Genomic RNA Replication in the Absence of Replication Organelles. Cell Rep 21:587-599.

46. Sun D, Wen X, Wang M, Mao S, Cheng A, Yang X, Jia R, Chen S, Yang $Q$, Wu Y, Zhu D, Liu M, Zhao X, Zhang S, Wang Y, Xu Z, Chen Z, Zhu L, Luo Q, Liu Y, Yu Y, Zhang L, Chen X. 2019. Apoptosis and Autophagy in Picornavirus Infection. Front Microbiol. Frontiers Media S.A.

47. Bird SW, Maynard ND, Covert MW, Kirkegaard K. 2014. Nonlytic viral spread enhanced by autophagy components. Proc Natl Acad Sci U S A 111:13081-6.

48. Tatematsu M, Funami K, Seya T, Matsumoto M. 2018. Extracellular RNA Sensing by Pattern Recognition Receptors. J Innate Immun. S. Karger AG.

49. Weber-Gerlach M, Weber F. 2016. Standing on three legs: antiviral activities of RIG-I against influenza viruses. Curr Opin Immunol 42:71-75.

50. Runge S, Sparrer KMJ, Lässig C, Hembach K, Baum A, García-Sastre A, Söding J, Conzelmann K-K, Hopfner K-P. 2014. In Vivo Ligands of MDA5 and RIG-I in Measles Virus-Infected Cells. PLoS Pathog 10:e1004081.

51. Ikegame S, Takeda M, Ohno S, Nakatsu Y, Nakanishi Y, Yanagi Y. 2010. Both RIG-I and MDA5 RNA helicases contribute to the induction of alpha/beta interferon in measles virus-infected human cells. J Virol 84:372-9.

52. Kato H, Takeuchi O, Mikamo-Satoh E, Hirai R, Kawai T, Matsushita K, Hiiragi A, Dermody TS, Fuiita T, Akira S. 2008. Length-dependent recognition of double-stranded ribonucleic acids by retinoic acid-inducible gene-I and melanoma differentiation-associated gene 5.J Exp Med.

53. Feng $\mathrm{Q}$ Hato SV V., Langereis MAA, Zoll J, Virgen-Slane R, Peisley A, Hur S, Semler BLL, van Rij RP, van Kuppeveld FJM. 2012. MDA5 Detects the Double-Stranded RNA Replicative Form in Picornavirus-Infected Cells. Cell Rep 2:1187-1196.

54. Francisco E, Suthar M, Gale M, Rosenfeld AB, Racaniello VR. 2019. Cell-type specificity and functional redundancy of RIG-I-like receptors in innate immune sensing of Coxsackievirus B3 and encephalomyocarditis virus. Virology 528:7-18.

55. Davis ME, Gack MU. 2015. Ubiquitination in the antiviral immune response. Virology 479-480:52-65.

56. Heaton SM, Borg NA, Dixit VM. 2016. Ubiquitin in the activation and attenuation of innate antiviral immunity. J Exp Med 213:1-13.

57. Gack MU, Shin YC, Joo C-H, Urano T, Liang C, Sun L, Takeuchi O, Akira S, Chen Z, Inoue S, Jung JU. 2007. TRIM25 RING-finger E3 ubiquitin ligase is essential for RIG-I-mediated antiviral activity. Nature 446:916-920.

58. Wies E, Wang MK, Maharaj NP, Chen K, Zhou S, Finberg RW, Gack MU. 2013. Dephosphorylation of the RNA Sensors RIG-I and MDA5 by the Phosphatase PPI Is Essential for Innate Immune Signaling. Immunity 38:437-449.

59. Paz S, Vilasco M, Arguello M, Sun Q, Lacoste J, Nguyen TL-A, Zhao T, Shestakova EA, Zaari S, Bibeau-Poirier A, Servant MJ, Lin R, Meurs EF, Hiscott J. 2009. Ubiquitin-regulated recruitment of IkappaB kinase epsilon to the MAVS interferen signaling adapter. Mol Cell Biol 29:3401

60. Xia Z-P, Sun L, Chen X, Pineda G, Jiang X, Adhikari A, Zeng W, Chen ZJ. 2009. Direct activation of protein kinases by unanchored polyubiquitin chains. Nature 461:114-119.

61. Deng L, Wang C, Spencer E, Yang L, Braun A, You J, Slaughter C, Pickart C, Chen ZJ. 2000. Activation of the IxB Kinase Complex by TRAF6 Requires a Dimeric Ubiquitin-Conjugating Enzyme Complex and a Unique Polyubiquitin Chain. Cell 103:351-361.

62. Paz S, Vilasco M, Werden SJ, Arguello M, Joseph-Pillai D, Zhao T, Nguyen TL-A, Sun Q, Meurs EF, Lin R, Hiscott J. 2011. A functional C-terminal TRAF3-binding site in MAVS participates in positive and negative regulation of the IFN antiviral response. Cell Res 21:895-910.

63. Zeng W, Xu M, Liu S, Sun L, Chen ZJ. 2009. Key Role of Ubc5 and Lysine-63 Polyubiquitination in Viral Activation of IRF3. Mol Cell 36:315-325.
64. Lamothe B, Besse A, Campos AD, Webster WK, Wu H, Darnay BG. 2007. Site-specific Lys-63-linked tumo necrosis factor receptor-associated factor 6 auto-ubiquitination is a critical determinant of I kappa B kinase activation. J Biol Chem 282:4102-12.

65. Tu D, Zhu Z, Zhou AY, Yun C, Lee K-E, Toms AV, Li Y, Dunn GP, Chan E, Thai T, Yang S, Ficarro SB, Marto JA, Jeon H, Hahn WC, Barbie DA, Eck MJ. 2013. Structure and Ubiquitination-Dependent Activation of TAN Binding Kinase 1. Cell Rep 3:747-758

66. Li S, Wang L, Berman M, Kong Y-Y, Dorf ME. 2011. Mapping a Dynamic Innate Immunity Protein Interaction Network Regulating Type I Interferon Production. Immunity 35:426-440.

67. Narayan K, Waggoner L, Pham ST, Hendricks GL, Waggoner SN, Conlon J, Wang JP, Fitzgerald KA, Kang J. 2014. TRIM13 is a negative regulator of MDA5-mediated type I interferon production. J Virol 88:10748-57.

68. Arimoto K, Takahashi H, Hishiki T, Konishi H, Fujita T, Shimotohno K. 2007. Negative regulation of the RIG-I signaling by the ubiquitin ligase RNF125. Proc Natl Acad Sci U S A 104:7500-5.

69. Lin M, Zhao Z, Yang Z, Meng Q, Tan P, Xie W, Qin Y, Wang R-F, Cui J. 2016. USP38 Inhibits Type I Interferon Signaling by Editing TBK1 Ubiquitination through NLRP4 Signalosome. Mol Cell 64:267-281.

70. Nakhaei P, Mesplede T, Solis M, Sun Q, Zhao T, Yang L, Chuang T-H, Ware CF, Lin R, Hiscott J. 2009. The E Ubiquitin Ligase Triad3A Negatively Regulates the RIG-I/MAVS Signaling Pathway by Targeting TRAF3 for Degradation. PLoS Pathog 5:e1000650.

71. Zhao W, Wang L, Zhang M, Yuan C, Gao C. 2012. E3 Ubiquitin Ligase Tripartite Motif 38 Negatively Regulate TLR-Mediated Immune Responses by Proteasomal Degradation of TNF Receptor-Associated Factor 6 in Macrophages. J Immunol 188:2567-2574

72. Young JA, Sermwittayawong D, Kim H-J, Nandu S, An N, Erdjument-Bromage H, Tempst P, Coscoy L, Winoto A. 2011. Fas-associated Death Domain (FADD) and the E3 Ubiquitin-Protein Ligase TRIM21 Interact to Negatively Regulate Virus-induced Interferon Production. J Biol Chem 286:6521-6531.

73. Higgs R, Gabhann JN, Larbi N Ben, Breen EP, Fitzgerald KA, Jefferies CA. 2008. The E3 Ubiquitin Ligase Ro52 Negatively Regulates IFN- $\beta$ Production Post-Pathogen Recognition by Polyubiquitin-Mediated Degradation fIRF3. I Immunol 181:1780-1786.

74. Maine GN, Mao X, Komarck CM, Burstein E. 2007. COMMDi promotes the ubiquitination of NF- $x \mathrm{~B}$ subunits through a cullin-containing ubiquitin ligase. EMBO J 26:436-447.

75. Schneider WM, Chevillotte MD, Rice CM. 2014. Interferon-Stimulated Genes: A Complex Web of Hos Defenses. Annu Rev Immunol.

76. Bisbal C, Silverman RH. 2007. Diverse functions of RNase L and implications in pathology. Biochimie 89:789-798.

77. Drappier M, Michiels T. 2015. Inhibition of the OAS/RNase L pathway by viruses. Curr Opin Virol 15:19-26.

78. Ezelle H, Malathi K, Hassel B. 2016. The Roles of RNase-L in Antimicrobial Immunity and the CytoskeletonAssociated Innate Response. Int $J$ Mol Sci 17:74.

79. Lai C, Struckhoff JJ, Schneider J, Martinez-Sobrido L, Wolff T, García-Sastre A, Zhang D-E, Lenschow DJ. 2009. Mice Lacking the ISG15 E1 Enzyme UbEiL Demonstrate Increased Susceptibility to both MouseAdapted and Non-Mouse-Adapted Influenza B Virus Infection. J Virol 83:1147-1151.

80. Ketscher L, Hannß R, Morales DJ, Basters A, Guerra S, Goldmann T, Hausmann A, Prinz M, Naumann R, Pekosz A, Utermöhlen O, Lenschow DJ, Knobeloch K-P. 2015. Selective inactivation of USP18 isopeptidas activity in vivo enhances ISG15 conjugation and viral resistance. Proc Natl Acad Sci 112:1577-1582.

81. Rahnefeld A, Klingel K, Schuermann A, Diny NL, Althof N, Lindner A, Bleienheuft P, Savvatis K, Respondek D, Opitz E, Ketscher L, Sauter M, Seifert U, Tschöpe C, Poller W, Knobeloch K-P, Voigt A. 2014. Ubiquitin-like protein ISG15 (interferon-stimulated gene of $15 \mathrm{kDa}$ ) in host defense against heart failure in a mouse model of virus-induced cardiomyopathy. Circulation 130:1589-600.

82. Lenschow DJ, Lai C, Frias-Staheli N, Giannakopoulos N V., Lutz A, Wolff T, Osiak A, Levine B, Schmidt RE, García-Sastre A, Leib DA, Pekosz A, Knobeloch KP, Horak I, Virgin IV HW. 2007. IFN-stimulated gene 15 functions as a critical antiviral molecule against influenza, herpes, and Sindbis viruses. Proc Natl Acad Sci U S A 104:371-6. 
83. Bogunovic D, Byun M, Durfee LA, Abhyankar A, Sanal O, Mansouri D, Salem S, Radovanovic I, Grant A V., Adimi P, Mansouri N, Okada S, Bryant VL, Kong XF, Kreins A, Velez MM, Boisson B, Khalilzadeh S, Ozcelik U, Darazam IA, Schoggins JW, Rice CM, Al-Muhsen S, Behr M, Vogt G, Puel A, Bustamante J, Gros P, Huibregtse Darazam IA, Schoggins JW, Rice CM, Al-Muhsen S, Behr M, Vogt G, Puel A, Bustamante J, Gros P, Huibregtse
JM, Abel L, Boisson-Dupuis S, Casanova JL. 2012. Mycobacterial disease and impaired IFN- $\gamma$ immunity in humans with inherited ISG15 deficiency. Science (80-) 337:1684-8.

84. Shi HX, Yang K, Liu X, Liu XY, Wei B, Shan YF, Zhu LH, Wang C. 2010. Positive Regulation of Interferon Regulatory Factor 3 Activation by Hercs via ISG15 Modification. Mol Cell Biol 30:2424-36.

85. Ganesan M, Poluektova LY, Tuma DJ, Kharbanda KK, Osna NA. 2016. Acetaldehyde Disrupts Interferon Alpha Signaling in Hepatitis C Virus-Infected Liver Cells by Up-Regulating USP18. Alcohol Clin Exp Res 40:2329-2338.

86. Kim M-J, Hwang S-Y, Imaizumi T, Yoo J-Y. 2008. Negative Feedback Regulation of RIG-I-Mediated Antiviral Signaling by Interferon-Induced ISG15 Conjugation. J Virol 82:1474-83.

87. Du Y, Duan T, Feng Y, Liu Q Lin M, Cui J, Wang R. 2018. LRRC25 inhibits type I IFN signaling by targeting ISG15-associated RIG-I for autophagic degradation. EMBO J 37:351-366.

88. Dzimianski J V., Scholte FEM, Bergeron É, Pegan SD. 2019. ISG15: it’s Complicated. J Mol Biol Sooz22836:30136-6.

89. Lemaire PA, Anderson E, Lary J, Cole JL. 2008. Mechanism of PKR Activation by dsRNA. J Mol Biol.

90. Bertolotti A, Zhang Y, Hendershot LM, Harding HP, Ron D. 2000. Dynamic interaction of BiP and ER stress transducers in the unfolded-protein response. Nat Cell Biol.

91. Walter P, Ron D. 2011. The unfolded protein response: From stress pathway to homeostatic regulation. Science (80-).

92. Dong J, Qiu H, Garcia-Barrio M, Anderson J, Hinnebusch AG. 2000. Uncharged tRNA activates GCN2 by displacing the protein kinase moiety from a bipartite tRNA-binding domain. Mol Cell 6:269-79.

93. Castilho BA, Shanmugam R, Silva RC, Ramesh R, Himme BM, Sattlegger E. 2014. Keeping the eIF2 alpha kinase Gcnz in check. Biochim Biophys Acta - Mol Cell Res.

94. Han AP, Yu C, Lu L, Fujiwara Y, Browne C, Chin G, Fleming M, Leboulch P, Orkin SH, Chen JJ. 2001. Hemeregulated eIF $2 \alpha$ kinase (HRI) is required for translational regulation and survival of erythroid precursors in iron deficiency. EMBO J.

95. Matts RL, Schatz JR, Hurst R, Kagen R. 1991. Toxic heavy metal ions activate the heme-regulated eukaryotic initiation factor- $2 \alpha$ kinase by inhibiting the capacity of hemin-supplemented reticulocyte lysates to reduce disulfide bonds. J Biol Chem 266:12695-702.

96. Chen JJ, London IM. 1995. Regulation of protein synthesis by heme-regulated eIF-2 $\alpha$ kinase. Trends Biochem Sci 20:105-8.

97. Panniers R, Henshaw EC. 1983. A GDP/GTP exchange factor essential for eukaryotic initiation factor 2 cycling in Ehrlich ascites tumor cells and its regulation by eukaryotic initiation factor 2 phosphorylation. I Biol Chem.

98. Rowlands a G, Panniers R, Henshaw EC. 1988. The catalytic mechanism of guanine nucleotide exchange factor action and competitive inhibition by phosphorylated eukaryotic initiation factor 2. J Biol Chem.

99. Pakos-Zebrucka K, Koryga I, Mnich K, Ljujic M, Samali A, Gorman AM. 2016. The integrated stress response. EMBO Rep 17:1374-1395.

100. Onomoto K, Jogi M, Yoo JS, Narita R, Morimoto S, Takemura A, Sambhara S, Kawaguchi A, Osari S, Nagata $\mathrm{K}$, Matsumiya T, Namiki H, Yoneyama M, Fujita T. 2012. Critical role of an antiviral stress granule containing RIG-I and PKR in viral detection and innate immunity. PLoS One 7:e43031.

101. Ng CS, Jogi M, Yoo J-S, Onomoto K, Koike S, Iwasaki T, Yoneyama M, Kato H, Fujita T. 2013. Encephalomyocarditis virus disrupts stress granules, the critical platform for triggering antiviral innate immune responses. J Virol 87:9511-22.

102. Reineke LC, Lloyd RE. 2015. The stress granule protein $\mathrm{G}_{3} \mathrm{BP} 1$ recruits protein kinase $\mathrm{R}$ to promote multiple innate immune antiviral responses. J Virol 89:2575-89.
103. Langereis MA, Feng $\mathrm{Q}$ van Kuppeveld FJ. 2013. MDAs localizes to stress granules, but this localization is not required for the induction of type I interferon. J Virol 87:6314-25.

104. Reineke LC, Kedersha N, Langereis MA, van Kuppeveld FJMM, Lloyd RE. 2015. Stress granules regulate double-stranded RNA-dependent protein kinase activation through a complex containing G3BP1 an Caprini. MBio 6:e02486-14.

105. Humoud MN, Doyle N, Royall E, Willcocks MM, Sorgeloos F, van Kuppeveld F, Roberts LO, Goodfellow IG, Langereis MA, Locker N. 2016. Feline Calicivirus Infection Disrupts Assembly of Cytoplasmic Stress Granules and Induces $\mathrm{G}_{3} \mathrm{BP}$ 1 Cleavage. J Virol 90:6489-501.

106. Garaigorta U, Heim MH, Boyd B, Wieland S, Chisari F V. 2012. Hepatitis C virus (HCV) induces formation of stress granules whose proteins regulate HCV RNA replication and virus assembly and egress. J Virol 86:11043-56.

107. Xia J, Chen X, Xu F, Wang Y, Shi Y, Li Y, He J, Zhang P. 2015. Dengue virus infection induces formation of G3BP1 granules in human lung epithelial cells. Arch Virol 160:2991-2999.

108. Bidet K, Dadlani D, Garcia-Blanco MA. 2014. G3BP1, G3BP2 and CAPRIN1 Are Required for Translation of Interferon Stimulated mRNAs and Are Targeted by a Dengue Virus Non-coding RNA. PLoS Pathog 10:e1004242.

109. Panas MD, Varjak M, Lulla A, Eng KE, Merits A, Karlsson Hedestam GB, McInerney GM. 2012. Sequestration of $\mathrm{G}_{3} \mathrm{BP}$ coupled with efficient translation inhibits stress granules in Semliki Forest virus infection. Mol Biol Cell 23:4701-12.

110. Scholte FEM, Tas A, Albulescu IC, Žusinaite E, Merits A, Snijder EJ, van Hemert MJ. 2015. Stress granule components $\mathrm{G}_{3} \mathrm{BP}$ and $\mathrm{G}_{3} \mathrm{BP} 2$ play a proviral role early in Chikungunya virus replication. J Virol 89:4457-69.

111. Fros JJ, Domeradzka NE, Baggen J, Geertsema C, Flipse J, Vlak JM, Pijlman GP. 2012. Chikungunya virus nsP blocks stress granule assembly by recruitment of $\mathrm{G}_{3} \mathrm{BP}$ into cytoplasmic foci. J Virol 86:10873-9.

112. Agol VI, Gmyl AP. 2010. Viral security proteins: counteracting host defences. Nat Rev Microbiol 8:867-78.

113. Villa-Komaroff L, Guttman N, Baltimore D, Lodishi HF. 1975. Complete translation of poliovirus RNA in a eukaryotic cell-free system. Proc Natl Acad Sci U S A 72:4157.

114. Bernstein HD, Sonenberg N, Baltimore D. 1985. Poliovirus mutant that does not selectively inhibit host cell protein synthesis. Mol Cell Biol 5:2913-23.

115. Grubman MJ, Zellner M, Wagner J. 1987. Antigenic comparison of the polypeptides of foot-and-mouth disease virus serotypes and other picornaviruses. Virology 158:133-140.

116. Haghighat A, Svitkin Y, Novoa I, Kuechler E, Skern T, Sonenberg N. 1996. The eIF4G-eIF4E complex is the target for direct cleavage by the rhinovirus $2 \mathrm{~A}$ proteinase. J Virol.

117. Devaney MA, Vakharia VN, Lloyd RE, Ehrenfeld E, Grubman MJ. 1988. Leader protein of foot-and-mouth disease virus is required for cleavage of the p220 component of the cap-binding protein complex. J Viro 62:4407-9.

118. Ventoso I, MacMillan SE, Hershey JW b., Carrasco L. 1998. Poliovirus 2A proteinase cleaves directly the eIF$4 \mathrm{G}$ subunit of eIF-4F complex. FEBS Lett 435:79-83.

119. Redondo N, Sanz MA, Welnowska E, Carrasco L. 2011. Translation without eiF2 promoted by poliovirus $2 A$ protease. PLoS One 6

120. Sanz MA, Welnowska E, Redondo N, Carrasco L. 2010. Translation Driven by Picornavirus IRES Is Hampered from Sindbis Virus Replicons: Rescue by Poliovirus 2A Protease. J Mol Biol 402:101-117.

121. Moral-López P, Alvarez E Redondo N Skern T Carrasco L. 2014. L protease from foot and mouth disease virus confers eIF2-independent translation for mRNAs bearing picornavirus IRES. FEBS Lett 588:4053-4059.

122. Kerekatte V, Keiper BD, Badorff C, Cai A, Knowlton KU, Rhoads RE. 1999. Cleavage of Poly(A)-binding protein by coxsackievirus $2 \mathrm{~A}$ protease in vitro and in vivo: another mechanism for host protein synthesis shutoff? J Virol 73:709-17.

123. Joachims M, Van Breugel PC, Lloyd RE. 1999. Cleavage of poly(A)-binding protein by enterovirus proteases concurrent with inhibition of translation in vitro. J Virol 73:718-27. 
124. Jen G, Detjen BM, Thach RE. 1980. Shutoff of HeLa cell protein synthesis by encephalomyocarditis virus and poliovirus: a comparative study. J Virol 35:150-6.

25. Aminev AG, Amineva SP, Palmenberg AC. 2003. Encephalomyocarditis viral protein $2 \mathrm{~A}$ localizes to nucleoli and inhibits cap-dependent mRNA translation. Virus Res.

126. Medvedkina OA, Scarlat IV, Kalinina NO, Agol VI. 1974. Virus-specific proteins associated with ribosomes of Krebs-II cells infected with encephalomyocarditis virus. FEBS Lett 39:4-8.

127. Groppo R, Palmenberg AC. 2007. Cardiovirus 2A protein associates with $40 \mathrm{~S}$ but not $80 \mathrm{~S}$ ribosome subunits during infection. J Virol 81:13067-74.

128. Zoll J, Galama JM, van Kuppeveld FJ, Melchers WJ. 1996. Mengovirus leader is involved in the inhibition of host cell protein synthesis. J Virol 70:4948-52.

129. Petty R V, Basta HA, Bacot-Davis VR, Brown BA, Palmenberg AC. 2014. Binding interactions between the encephalomyocarditis virus leader and protein 2A. J Virol 88:13503-9.

130. Ricour C, Delhaye S, Hato S V., Olenyik TD, Michel B, van Kuppeveld FJM, Gustin KE, Michiels T. 2009. Inhibition of mRNA export and dimerization of interferon regulatory factor 3 by Theiler's virus leader protein. J Gen Virol 90:177-186.

131. Porter FW, Bochkov YA, Albee AJ, Wiese C, Palmenberg AC. 2006. A picornavirus protein interacts with RanGTPase and disrupts nucleocytoplasmic transport. Proc Natl Acad Sci U S A 103:12417-22.

132. Lidsky P V, Hato S, Bardina M V, Aminev AG, Palmenberg AC, Sheval E V, Polyakov VY, van Kuppeveld FJM, Agol VI. 2006. Nucleocytoplasmic traffic disorder induced by cardioviruses. J Virol 80:2705-17.

133. Porter FW, Palmenberg AC. 2009. Leader-induced phosphorylation of nucleoporins correlates with nuclear trafficking inhibition by cardioviruses. J Virol 83:1941-51.

134. Bardina M V, Lidsky P V, Sheval E V, Fominykh K V, van Kuppeveld FJM, Polyakov VY, Agol VI. 2009. Mengovirus-induced rearrangement of the nuclear pore complex: hijacking cellular phosphorylation machinery. J Virol 83:3150-61.

135. Belov GA, Lidsky P V, Mikitas O V, Egger D, Lukyanov KA, Bienz K, Agol VI. 2004. Bidirectional increase in permeability of nuclear envelope upon poliovirus infection and accompanying alterations of nuclear pores. J Virol 78:10166-77.

136. Park N, Skern T, Gustin KE. 2010. Specific cleavage of the nuclear pore complex protein Nup62 by a viral protease. J Biol Chem 285:28796-805.

137. Park N, Katikaneni P, Skern T, Gustin KE. 2008. Differential targeting of nuclear pore complex proteins in poliovirus-infected cells. J Virol 82:1647-55.

138. Watters K, Palmenberg AC. 2011. Differential processing of nuclear pore complex proteins by rhinovirus $2 \mathrm{~A}$ proteases from different species and serotypes. J Virol 85:10874-83.

139. Gustin KE, Sarnow P. 2001. Effects of poliovirus infection on nucleo-cytoplasmic trafficking and nuclear pore complex composition. EMBO J 20:240-9.

140. Gustin KE, Sarnow P. 2002. Inhibition of Nuclear Import and Alteration of Nuclear Pore Complex Composition by Rhinovirus. J Virol.

141. Rubinstein SJ, Hammerle T, Wimmer E, Dasgupta A. 1992. Infection of HeLa cells with poliovirus results in modification of a complex that binds to the rRNA promoter. J Virol.

142. Yalamanchili P, Dasgupta A, Harris K, Wimmer E. 1996. Inhibition of basal transcription by poliovirus: A virus-encoded protease ( $3 \mathrm{Cpro}$ ) inhibits formation of TBP-TATA box complex in vitro. J Virol.

143. Kliewer S, Dasgupta A. 1988. An RNA polymerase II transcription factor inactivated in poliovirus-infected cells copurifies with transcription factor TFIID. Mol Cell Biol.

144. Kundu P, Raychaudhuri S, Tsai W, Dasgupta A. 2005. Shutoff of RNA Polymerase II Transcription by Poliovirus Involves $3 \mathrm{C}$ Protease-Mediated Cleavage of the TATA-Binding Protein at an Alternative Site: Incomplete Shutoff of Transcription Interferes with Efficient Viral Replication. J Virol.
145. Baneriee R, Weidman MK, Navarro S, Comai L, Dasgupta A. 2005. Modifications of both selectivity factor and upstream binding factor contribute to poliovirus-mediated inhibition of RNA polymerase I transcription. Gen Virol.

146. Weidman MK, Yalamanchili P, Ng B, Tsai W, Dasgupta A. 2001. Poliovirus 3 C protease-mediated degradation of transcriptional activator p53 requires a cellular activity. Virology.

147. Flather D, Semler BL. 2015. Picornaviruses and nuclear functions: Targeting a cellular compartment distinct from the replication site of a positive-strand RNA virus. Front Microbiol.

148. de Los Santos T, Diaz-San Segundo F, Grubman MJ. 2007. Degradation of nuclear factor kappa B during foot-and-mouth disease virus infection. J Virol 81:12803-12815.

149. de los Santos T, Diaz-San Segundo F, Zhu J, Koster M, Dias CCA, Grubman MJ. 2008. A Conserved Domain in the Leader Proteinase of Foot-and-Mouth Disease Virus Is Required for Proper Subcellular Localization and Function. J Virol 83:1800-10.

150. Piñeiro D, Ramajo J, Bradrick SS, Martínez-Salas E. 2012. Gemin5 proteolysis reveals a novel motif to identify protease targets. Nucleic Acids Res 40:4942-4953.

151. Medina GN, Knudsen GM, Greninger AL, Kloc A, Díaz-San Segundo F, Rieder E, Grubman MI, DeRisi JL, de los Santos T. 2017. Interaction between FMDV Lpro and transcription factor ADNP is required for optimal viral replication. Virology 505:12-22.

152. Feng Q, Langereis MA, Lork M, Nguyen M, Hato S V, Lanke K, Emdad L, Bhoopathi P, Fisher PB, Lloyd RE, van Kuppeveld FJM. 2014. Enterovirus 2Apro targets MDA5 and MAVS in infected cells. J Virol 88:3369-78.

153. Wang B, Xi X, Lei X, Zhang X, Cui S, Wang J, Jin Q, Zhao Z. 2013. Enterovirus 71 Protease 2Apro Target MAVS to Inhibit Anti-Viral Type I Interferon Responses. PLoS Pathog 9:e1003231.

154. Drahos J, Racaniello VR. 2009. Cleavage of IPS-1 in cells infected with human rhinovirus. J Virol 83:11581-7.

155. Mukherjee A, Morosky SA, Delorme-Axford E, Dybdahl-Sissoko N, Oberste MS, Wang T, Coyne CB. 2011. The coxsackievirus B 3 Cpro protease cleaves MAVS and TRIF to attenuate host type I interferon and apoptotic signaling. PLoS Pathog 7:e1001311.

156. Pang L, Yuan X, Shao C, Li M, Wang Y, Wang H, Xie G, Xie Z, Yuan Y, Zhou D, Sun X, Zhang Q, Xin Y, Li D, Duan Z. 2017. The suppression of innate immune response by human rhinovirus C. Biochem Biophys Res Commun 490:22-28.

157. Lei $\mathrm{X}$, Xiao $\mathrm{X}$, Xue $\mathrm{Q}$ Jin $\mathrm{Q}$, He B, Wang J. 2013. Cleavage of interferon regulatory factor 7 by enterovirus 71 $3 \mathrm{C}$ suppresses cellular responses. J Virol 87:1690-8.

158. Xiang Z, Liu L, Lei X, Zhou Z, He B, Wang J. 2016. 3C Protease of Enterovirus D68 Inhibits Cellular Defense Mediated by Interferon Regulatory Factor 7. J Virol 90:1613-21.

159. Hung HC, Wang HC, Shih SR, Teng IF, Tseng CP, Hsu JTA. 2011. Synergistic inhibition of enterovirus 7 replication by interferon and rupintrivir. J Infect Dis.

160. Barral PM, Sarkar D, Fisher PB, Racaniello VR. 2009. RIG-I is cleaved during picornavirus infection. Virology 391:171-176.

161. Zhang W, Zhang L, Wu Z, Tien P. 2014. Differential interferon pathway gene expression patterns in Rhabdomyosarcoma cells during Enterovirus 71 or Coxsackievirus A16 infection. Biochem Biophys Res Commun 447:550-55

162. Lu J, Yi L, Zhao J, Yu J, Chen Y, Lin MC, Kung H-F, He M-L. 2012. Enterovirus 71 disrupts interferon signaling by reducing the level of interferon receptor 1.J Virol 86:3767-76.

163. Wang C, Sun M, Yuan X, Ji L, Jin Y, Cardona CJ, Xing Z. 2017. Enterovirus 71 suppresses interferon responses by blocking Janus kinase (JAK)/signal transducer and activator of transcription (STAT) signaling through inducing karyopherin- $\alpha 1$ degradation. J Biol Chem 292:10262-10274.

164. de Los Santos T, de Avila Botton S, Weiblen R, Grubman MJ. 2006. The leader proteinase of foot-and-mout disease virus inhibits the induction of beta interferon $\mathrm{mRNA}$ and blocks the host innate immune response. Virol 80:1906-14. 
165. Wang D, Fang L, Luo R, Ye R, Fang Y, Xie L, Chen H, Xiao S. 2010. Foot-and-mouth disease virus leader proteinase inhibits dsRNA-induced type I interferon transcription by decreasing interferon regulatory proteinase inhibits dsRNA-induced type I interferon transcription
factor $3 / 7$ in protein levels. Biochem Biophys Res Commun 399:72-78.

166. Wang D, Fang L, Li P, Sun L, Fan J, Zhang Q, Luo R, Liu X, Li K, Chen H, Chen Z, Xiao S. 2011. The Leader Proteinase of Foot-and-Mouth Disease Virus Negatively Regulates the Type I Interferon Pathway by Acting as a Viral Deubiquitinase. J Virol 85:3758-3766.

167. Wang D, Fang L, Li K, Zhong H, Fan J, Ouyang C, Zhang H, Duan E, Luo R, Zhang Z, Liu X, Chen H, Xiao S. 2012. Foot-and-mouth disease virus $3 \mathrm{C}$ protease cleaves NEMO to impair innate immune signaling. J Virol 86:9311-9322.

168. Du Y, Bi J, Liu J, Liu X, Wu X, Jiang P, Yoo D, Zhang Y, Wu J, Wan R, Zhao X, Guo L, Sun W, Cong X, Chen L, Wang J. 2014. 3Cpro of foot-and-mouth disease virus antagonizes the interferon signaling pathway by blocking STAT1/STAT2 nuclear translocation. J Virol 88:4908-20.

169. Hato S V., Sorgeloos F, Ricour C, Zoll J, Melchers WJG, Michiels T, van Kuppeveld FJM. 2010. Differential IFN- $\alpha / \beta$ production suppressing capacities of the leader proteins of mengovirus and foot-and-mouth disease virus. Cell Microbiol 12:310-317.

170. Hato S V, Ricour C, Schulte BM, Lanke KHW, de Bruijn M, Zoll J, Melchers WJG, Michiels T, van Kuppeveld FJM. 2007. The mengovirus leader protein blocks interferon-alpha/beta gene transcription and inhibits activation of interferon regulatory factor 3. Cell Microbiol 9:2921-2930.

171. Paul S, Michiels T. 2006. Cardiovirus leader proteins are functionally interchangeable and have evolved to adapt to virus replication fitness. J Gen Virol 87:1237-1246.

172. van Pesch V, van Eyll O, Michiels T, Lipton HL, Rundell MK. 2001. The leader protein of Theiler's virus inhibits immediate-early alpha/beta interferon production. J Virol 75:7811-7.

173. Stavrou S, Feng Z, Lemon SM, Roos RP. 2010. Different Strains of Theiler's Murine Encephalomyelitis Virus Antagonize Different Sites in the Type I Interferon Pathway. J Virol 75:7811-7817.

174. Sorgeloos F, Jha BK, Silverman RH, Michiels T. 2013. Evasion of Antiviral Innate Immunity by Theiler's Virus L* Protein through Direct Inhibition of RNase L. PLoS Pathog 9:e1003474.

175. White JP, Cardenas AM, Marissen WE, Lloyd RE. 2007. Inhibition of Cytoplasmic mRNA Stress Granule Formation by a Viral Proteinase. Cell Host Microbe 2:295-305.

176. Redondo N, Sanz MA, Welnowska E, Carrasco L. 2011. Translation without eIF2 Promoted by Poliovirus 2A Protease. PLoS One 6:e25699.

177. Black TL, Safer B, Hovanessian A, Katze MG. 1989. The cellular 68,000-Mr protein kinase is highly autophosphorylated and activated yet significantly degraded during poliovirus infection: implications for translational regulation. J Virol 63:2244-51.

178. Fung G, Ng CS, Zhang J, Shi J, Wong J, Piesik P, Han L, Chu F, Jagdeo J, Jan E, Fujita T, Luo H. 2013. Production of a dominant-negative fragment due to $\mathrm{G}_{3} \mathrm{BPI}$ cleavage contributes to the disruption of mitochondriaassociated protective stress granules during CVB3 infection. PLoS One 8:e79546.

179. Zhang Y, Yao L, Xu X, Han H, Li P, Zou D, Li X, Zheng L, Cheng L, Shen Y, Wang X, Wu X, Xu J, Song B, Xu S, Zhang H, Cao H. 2018. Enterovirus 71 inhibits cytoplasmic stress granule formation during the late stage of infection. Virus Res 255:55-67.

180. Borghese F, Michiels T. 2011. The Leader Protein of Cardioviruses Inhibits Stress Granule Assembly. J Virol 85:9614-9622.

181. Rabouw HH, Langereis MA, Knaap RCM, Dalebout TJ, Canton J, Sola I, Enjuanes L, Bredenbeek PJ, Kikkert M, de Groot RJ, van Kuppeveld FJM. 2016. Middle East Respiratory Coronavirus Accessory Protein 4a Inhibits PKR-Mediated Antiviral Stress Responses. PLoS Pathog 12:e1005982.

182. Borghese F, Sorgeloos F, Cesaro T, Michiels T. 2019. The Leader Protein of Theiler's Virus Prevents the Activation of PKR. J Virol 93.

183. Rai DK, Lawrence P, Kloc A, Schafer E, Rieder E. 2015. Analysis of the interaction between host factor Sam68 and viral elements during foot-and-mouth disease virus infections. Virol J 12:224. 


\section{Chapter 2}

FMDV leader protease cleaves G3BP1 and G3BP2 and inhibits stress granule formation

Linda J. Visser ', Gisselle N. Medina 2, Huib H. Rabouw ', Raoul J. de Groot ', Martijn A. Langereis ${ }^{1}$, Teresa de los Santos ${ }^{2}$, Frank J.M. van Kuppeveld ${ }^{1, *}$

${ }^{1}$ Virology Division, Department of Infectious Diseases and Immunology, Faculty of Veterinary Medicine, Utrecht University, The Netherlands

${ }^{2}$ United States Department of Agriculture, Agricultural Research Service, Foreign Animal Disease Research Unit, Plum Island Animal Disease Center, Orient, NY, USA

"Corresponding author. 


\section{Abstract}

Like other viruses, the picornavirus foot-and-mouth disease virus (FMDV, genus Aphthovirus), one of the most notorious pathogens in global livestock industry, needs to navigate antiviral host responses to establish an infection. There is substantial insight into how FMDV suppresses the type I IFN response, but it is largely unknown whether and how FMDV modulates the integrated stress response. Here, we show that the stress response is suppressed during FMDV infection. Using a chimeric recombinant encephalomyocarditis virus (EMCV), in which we functionally replaced the endogenous stress response antagonist by FMDV $\mathrm{L}^{\text {pro }}$ or $3 \mathrm{C}^{\text {pro }}$, we demonstrate an essential role for $\mathrm{L}^{\text {pro }}$ in suppressing stress granule (SG) formation. Consistently, infection with a recombinant FMDV lacking $\mathrm{L}^{\text {pro }}$ resulted in SG formation. Additionally, we show that $\mathrm{L}^{\text {pro }}$ cleaves the known SG scaffold proteins G3BP1 and $\mathrm{G}_{3} \mathrm{BP} 2$, but not TIA-1. We demonstrate that the closely related equine rhinitis $\mathrm{A}$ virus (ERAV) L $\mathrm{L}^{\text {pro }}$ also cleaves $\mathrm{G}_{3} \mathrm{BP} 1$ and $\mathrm{G}_{3} \mathrm{BP} 2$ and also suppresses $\mathrm{SG}$ formation, indicating that these abilities are conserved amongst aphthoviruses. Neither FMDV or ERAV pro $^{\text {pro }}$ interfered with phosphorylation of PKR or eIF2 $\alpha$, indicating that $L^{\text {pro }}$ does not affect SG formation by inhibiting the PKR-triggered signaling cascade. Taken together, our data suggest that aphthoviruses actively target scaffolding proteins $\mathrm{G}_{3} \mathrm{BP}_{1}$ and $\mathrm{G}_{3} \mathrm{BP}_{2}$ and antagonize SG formation to modulate the integrated stress response.

\section{Importance}

The picornavirus foot and mouth disease virus (FMDV) is a notorious animal pathogen that puts a major economic burden on global life stock industry. Outbreaks have significant consequences for animal health and product safety. Like many other viruses, FMDV must manipulate antiviral host responses to establish infection. Upon infection, viral dsRNA is detected which results in activation of the PKR-mediated stress response, leading to a stop in cellular and viral translation and the formation of stress granules (SG), which are thought to have antiviral properties. Here we show that FMDV can suppress SG formation via its leader protease $\left(\mathrm{L}^{\mathrm{pro}}\right)$. Simultaneously we observed that $\mathrm{L}^{\text {pro }}$ can cleave the SG scaffolding proteins $\mathrm{G}_{3} \mathrm{BP} 1$ and $\mathrm{G}_{3} \mathrm{BP} 2$. Understanding the molecular mechanisms of FMDV's antiviral host response evasion strategies may help to develop countermeasures to control FMDV infections in the future.

\section{Introduction}

Innate antiviral responses represent the first line of defense against viral infections. Cellular antiviral signaling is initiated upon recognition of pathogen-associated molecular patterns (PAMPs) by the host. Cytosolic viral RNA can be detected by the RIG-I like receptors (RLRs). Upon recognition of viral RNA, RLRs activate signal transduction pathways resulting in the production and secretion of type I interferons (IFN- $\alpha / \beta$ ) and pro-inflammatory cytokines. Secreted IFN- $\alpha / \beta$ triggers the transcription of interferon-stimulated genes (ISGs), thereby generating an antiviral state in both infected and surrounding cells that limits virus spread (1).

Besides the IFN- $\alpha / \beta$ pathway, the cellular integrated stress response (ISR) has been increasingly recognized as an antiviral pathway (2). Cells can halt translation upon different kind of cellular stress, including viral infection. Double-stranded (ds) RNAdependent protein kinase (PKR), an ISG, detects cytosolic viral double-stranded RNA (dsRNA) and activates the stress response in virus-infected cells (3). Detection of dsRNA by PKR results in structural rearrangements in PKR leading to auto-phosphorylation. Next, activated PKR phosphorylates the alpha-subunit of eIF2. Phosphorylation of eIF2 $\alpha$ halts translation, thereby interfering with synthesis of viral proteins and virus propagation (3). Upon halted translation, specific nucleating factors bind specific stalled mRNA-protein complexes (mRNPs) to form cytoplasmic aggregates known as stress granules (SGs). These nucleating factors include Ras GTPase-activating protein-binding proteins 1 and 2 (G3BP1 and G3BP2), T cell-restricted intracellular antigen 1 (TIA-1) and the closely related TIAR, and multiple other factors (e.g. Caprini and Sam68). SGs are highly dynamic cytoplasmic aggregates. Scaffold proteins continuously cycle between the cytosol and SGs, facilitating mRNA triage, in order to direct translation towards resolving the cellular stress $(2,4)$.

SGs have been proposed to act as a platform for antiviral signaling $(2,4-7)$. The local enrichment of nucleating factors and mRNPs most likely constitutes a warning signal in the cell, recruiting numerous signaling molecules to the SGs (4). These include several signaling molecules involved in the innate antiviral response, such as RIG-I (8), MDA5 (9), TRAF2 (10) and PKR (5). Despite these observations, the importance and role of SGs as an antiviral signaling platform are still poorly understood. The most important argument that supports an antiviral role of SGs is the fact that many viruses have developed antagonistic mechanisms that interfere with SG assembly. These mechanisms include inhibition of PKR, cleavage of SG scaffold proteins by viral proteases, and sequestration of SG scaffold proteins by viral proteins $(2,11-17)$. 
Picornaviruses, a large family of small, non-enveloped viruses that contain a single-stranded 7-8 kb RNA genome of positive polarity, actively antagonize both RLR signaling and the stress response. Viruses belonging to the genus Enterovirus, best known for poliovirus, actively suppress RLR signaling through their viral proteases, $2 \mathrm{~A}^{\text {pro }}$ and $3 \mathrm{C}^{\text {pro }}$, which cleave multiple proteins in the RLR signaling pathway (18-22). $2 \mathrm{~A}^{\text {pro }}$ and $3 \mathrm{C}^{\text {pro }}$ also cleave signaling molecules of the IFN- $\alpha / \beta$ signaling pathway to prevent the expression of ISGs (23). Enteroviruses also actively antagonizes the cellular stress response, as SGs rapidly dissolve as infection progresses. This observation has been linked to the cleavage of the SG scaffold protein G3BPI by the viral $3 \mathrm{C}^{\text {pro }}(24)$, although other viral proteins have also been suggested to affect $S G$ formation (25). Similarly, caliciviruses encode NS6, a 3C-like protease, which also cleaves G3BP1 to prevent SG assembly (11). Members of the genus Cardiovirus (e.g. encephalomyocarditis virus (EMCV) and Theiler's virus) encode the leader protein, one antagonist that suppresses both RLR signaling and the stress response pathway $(9,18,26,27)$. The mechanism of action of the cardiovirus leader protein, which lacks proteolytic activity, is largely unknown. Besides the leader protein, the $3 \mathrm{C}^{\text {pro }}$ of cardioviruses has been implicated in suppressing $\mathrm{SG}$ formation by cleaving G3BP1 (28), although this was not observed in another study (26).

Another well-known picornavirus is foot-and-mouth disease virus (FMDV), which is one of the most notorious pathogens in global livestock industry. FMDV infections are well known for their devastating effects on animal health and product safety and can have a huge economic impact. FMDV is a member of the genus Aphthovirus, which also contains bovine rhinitis $A$ and $B$ viruses, and equine rhinitis A virus (ERAV). Like cardioviruses, aphthoviruses encode a leader protein. FMDV leader is a papain-like protease ( $\mathrm{L}^{\mathrm{pro}}$ ) that is best known for cleaving translation initiation factor eIF4G, thereby shutting off host mRNA translation (29). Efficient spread of FMDV infection in vivo relies on $\mathrm{L}^{\text {pro }}(30)$. Besides causing translational shutoff of host proteins, $L^{\text {pro }}$ also suppresses the induction of IFN- $\alpha / \beta$ (31). For this, several mechanisms have been proposed. $L^{\text {pro }}$ causes the degradation of NFKB subunit p65/RelA (32), decreases the levels of interferon regulatory factor 3 (IRF3) and IRF7 (33), and it has been proposed to possess deubiquitinase (DUB) and deISGylase activities $(34,35)$. Having DUB activity is a common strategy for viruses to manipulate IFN signaling pathways (36). The $3 \mathrm{C}^{\text {pro }}$ of aphthoviruses has also been implicated in suppressing IFN- $\alpha / \beta$ production by cleaving $\mathrm{NFKB}$ essential modulator (NEMO), an adaptor protein that is essential for activating NFKB and IRF signaling pathways (37) and interfering with the JAK/STAT signaling pathways by manipulating the STAT1/STAT2 nuclear translocation (38).

Little is known about whether and how aphthoviruses suppress the cellular stress response. Here, we demonstrate that aphthoviruses can suppress the cellular stress response through the action of the viral protease $\mathrm{L}^{\text {pro }}$, and we identify SG scaffold proteins $\mathrm{G}_{3} \mathrm{BP} 1$ and $\mathrm{G}_{3} \mathrm{BP} 2$ as new substrates of $\mathrm{L}^{\text {pro }}$.

\section{Results}

The stress response pathway is suppressed during FMDV infection. To investigate whether FMDV antagonizes the stress response pathway, we infected LFPK cells that stably express the integrin $\alpha v \beta 6$ (LFPK $\alpha v \beta 6$ cells), the entry receptor of FMDV (39), with FMDV A12 and performed immune fluorescence staining to visualize SG formation (Fig IA). As a positive control, cells were treated with sodium arsenite, a known activator of the stress response pathway (Fig. 1B). SGs were visualized by anti-G3BP1 staining, while viral replication was monitored by staining for the capsid protein VP1. In LFPK $\alpha v \beta 6$ cells FMDV replication is relatively fast, virus production and release can be observed as early as $5 \mathrm{hrs}$ post infection (hpi) (40). Consistently, we observed a clear accumulation of VP1 both at 4 and 6 hpi, indicative of efficient viral replication. Notwithstanding this, only a few G3BP1 foci were detected in the infected cells. In contrast, arsenite treatment of the cells led to extensive SG formation (Fig. 1B). These data show that FMDV infection does not induce SG formation. To investigate whether the virus actively suppresses SG formation, we infected LFPK $\alpha v \beta 6$ cells with FMDV A12 and added sodium arsenite during the last 30 min of infection (Fig. 1C). No SGs were observed in infected cells, whereas many SGs were observed in uninfected neighboring cells. Similar observations were made using IBRS-2 cells (Fig. ID), providing further support that FMDV actively antagonizes SG formation.

FMDV and ERAV L pro $^{\text {ro }}$ suppress SG formation when introduced into a recombinant EMCV containing an inactive stress antagonist. To determine whether the viral proteases are involved in suppressing SG formation, we generated recombinant EMCVs harboring FMDV L pro or $3 C^{\text {pro }}$ at the start of the ORF of EMCV-L $\mathrm{L}^{\mathrm{Zn}}$ (EMCV-FL and EMCV-F3C) (Fig 2A). EMCV- $\mathrm{L}^{\mathrm{Zn}}$ is a mutant $\mathrm{EMCV}$ (strain mengovirus) in which the antagonistic functions of the leader (L) protein are abolished by inactivating mutations in the zinc finger domain of $\mathrm{L}$. This virus no longer suppresses the IFN- $\alpha / \beta$ or stress response $(9,18,41,42)$, and therefore provides a tool to study the antagonistic function of an heterologous viral protein (18, 43). The inserted proteases were flanked at their $\mathrm{N}$-terminus by the first 6 amino acids of EMCV L to ensure efficient translation $(44,45)$, and an EMCV $3 C^{\text {pro }}$ cleavage site at their $\mathrm{C}$-terminus for efficient viral polyprotein processing. To determine whether the function of FMDV proteases is conserved in other aphthoviruses, we also constructed chimeric viruses encoding $\mathrm{L}^{\text {pro }}$ and $3 \mathrm{C}^{\text {pro }}$ of ERAV (EMCV-EL and EMCV-E3C). After recovering these chimeric EMCVs, we characterized viral replication kinetics by RT-qPCR analysis of EMCV viral RNA (vRNA) levels at various time points (Fig. 2B). As described previously (18, 41), mutation of the zinc-finger domain of EMCV L slightly reduced vRNA levels. Heterologous expression of either FMDV or ERAV L similar as those of wild-type EMCV, whereas expression of $3 C^{\text {pro }}$ (EMCV-F3C and EMCVE3C) did not affect vRNA levels. 

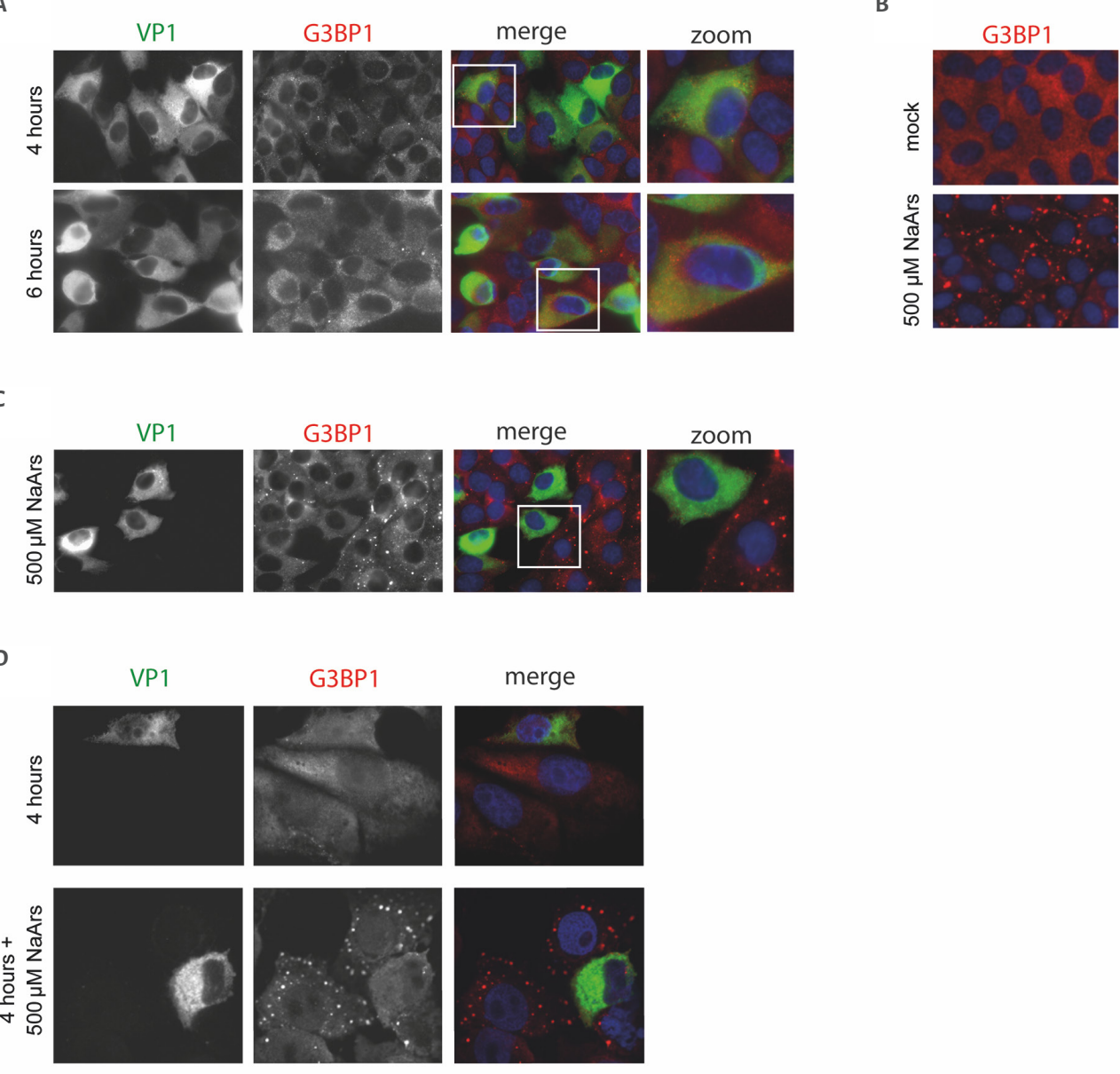

Figure 1: FDMV suppresses SG formation. (A) LFPK $\alpha v \beta 6$ cells were infected with FMDV-A12, fixed at 4 and 6 hpi and immunofluorescence staining was performed for the viral capsid protein $\mathrm{VP} 1$ (green) and the $\mathrm{SC}$ marker $\mathrm{C}_{3} \mathrm{BP}_{1}$ (red). (B) LFPK $\alpha v \beta 6$ cells were treated with $500 \mathrm{uM}$ sodium arsenite for $30 \mathrm{~min}$ to induce SC formation (as previously described $(24,26)$ ) which were subsequently visualized by immunofluorescence staining for the $S_{C}$ marker $\mathrm{C}_{3} \mathrm{BP}_{1}$ (red). (C) LFPK $\alpha v \beta 6$ cells were infected with FMDV-A12, treated with $500 \mu \mathrm{M}$ sodium arsenite for the last $30 \mathrm{~min}$ to induce SC formation, and fixed at $4 \mathrm{hpi}$. Immunofluorescence staining was performed for the viral capsid protein VP1 (green) and the SC marker $\mathrm{C}_{3} \mathrm{BP}_{1}$ (red). (D) Similar as (C) but using IBRS-2 cells.

Subsequently, we infected HeLa cells with these recombinant EMCVs and visualized SGs at 6 hpi using immune fluorescence microscopy. The SGs were visualized using anti-G3BP1, anti-G3BP2 and anti-TIA-1 staining. Consistent with previous results (9), EMCV infection suppressed SG formation, while EMCV- $\mathrm{L}^{\mathrm{Zn}}$ has lost this ability (Fig $2 \mathrm{C}$, top two rows). Compared to EMCV-L $\mathrm{L}^{\mathrm{Zn}}$, infection with EMCV-FL or EMCV-EL resulted in noticeably fewer and smaller SGs. In contrast, we observed no effect of $3 \mathrm{C}^{\text {pro }}$ on SG formation (Fig. $2 \mathrm{C}$ ). Similar observations were made at 8 hpi (data not shown). Quantification of SG size and number of
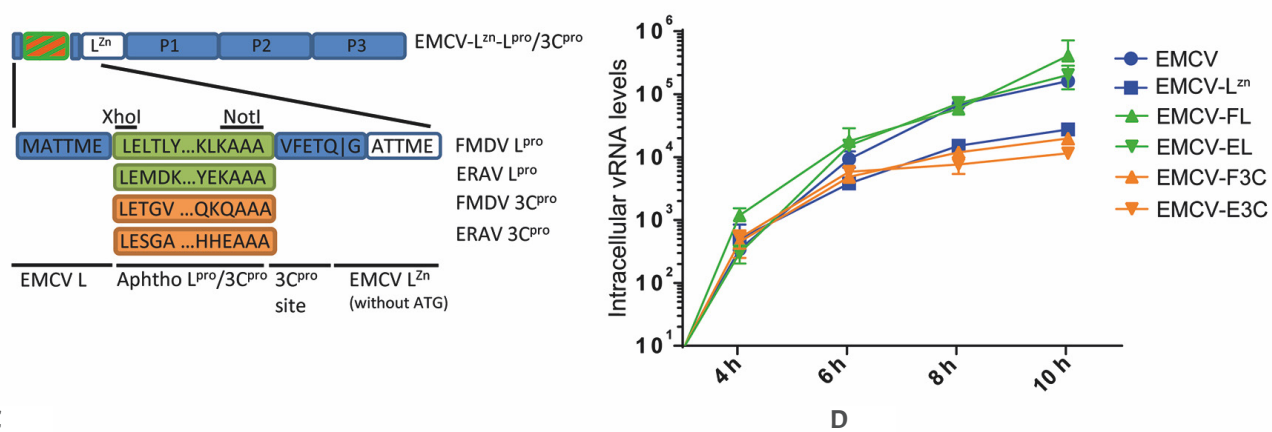

c
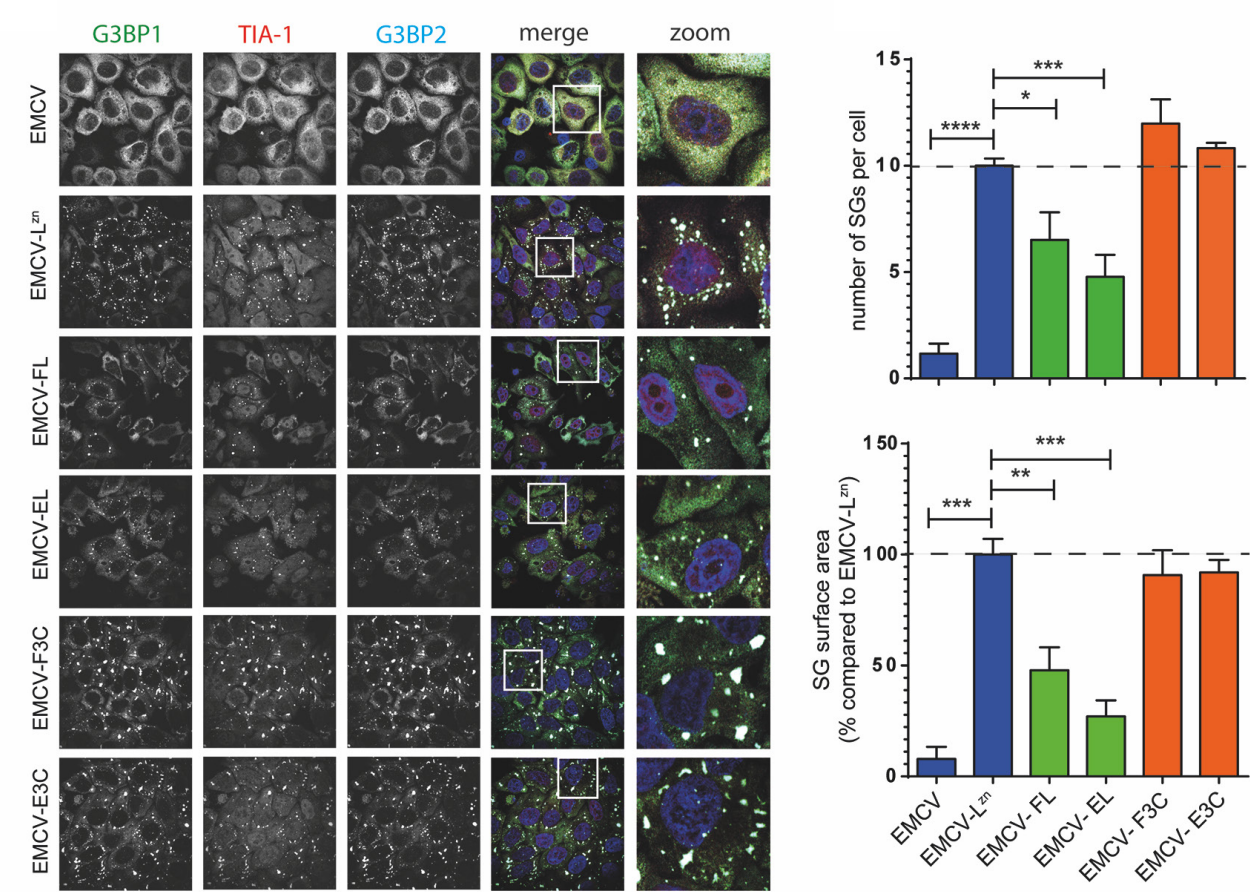

Figure 2: Aphthovirus Lpro suppresses SG formation. (A) Schematic representation of viral genome of chimeric EMC viruses that were constructed for this study. The endogenous stress response antagonist (Leader) has been inactivated by pos that were constructed for this study. The endogenous stress response antagonist (Leader) has been inactivated by point mutations in its $\mathrm{Zn}$-finger domain ( $19 \mathrm{~A} / \mathrm{C} 22 \mathrm{~A})$, and subsequently the genes encoding $\mathrm{L}^{\text {pro }}$ and $3^{\mathrm{C} \text { ro }}$ were introduced at the 5 ' end of the EMCV open reading frame. (B) HeLa R19 cells were infected at MOI 10 with the indicated primers. (C) Hela R19 cells were infected at MOl 10 with the indicated chimeric EMCVs and fixed at 6 hpi Subsequent

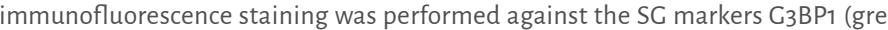
Quantification of (C) in which the number of SGs and SG size was analyzed for at least 50 cells per condition. One-way ANOVA with Bonferroni post-hoc test was used to determine statistical significance $(" p<0.05, " * * 0<0.01$, , **** $p<0.001$ ***** $p<0.0001$ ). 
SGs showed a consistent decrease in both parameters upon the heterologous expression of $L^{\text {pro }}$ (EMCV-FL and EMCV-EL), but not upon the expression of $3 C^{\text {pro }}$ (EMCV-F3C and EMCVE3C) (Fig 2D). Intriguingly, the viruses that have the ability to suppress SG formation (EMCVFL and EMCV-EL) also replicated to levels similar as wt EMCV, while the viruses that failed to do so (EMCV-F3C and EMCV-E3C) replicated to levels similar as EMCV- $\mathrm{L}^{\mathrm{Zn}}$. This suggests that the ability of $\mathrm{L}^{\text {pro }}$ to suppress $\mathrm{SG}$ formation, likely combined with its ability to suppress the type I IFN response, is beneficial to the replication of EMCV-FL and EMCV-EL.

To determine if the inhibitory effect of $\mathrm{L}^{\text {pro }}$ on $\mathrm{SG}$ formation is dependent on $\mathrm{L}^{\text {pro's }} \mathrm{S}$ catalytic activity, we generated an EMCV encoding a catalytically inactive FMDV $L^{\text {pro }}$ (EMCV-FL C51A) and investigated the ability of this virus to suppress SG formation (Fig 3). HeLa cells were infected with EMCV-L ${ }^{\mathrm{Zn}}, \mathrm{EMCV}-\mathrm{FL}$ or EMCV-FL C51A and SGs were visualized by immune fluorescence staining against $\mathrm{G}_{3} \mathrm{BP} 1$, eIF3 and G3BP2. Infection with EMCV-FL C51A resulted in SG formation (Fig 3A) and the SGs were of usual size (Fig. 3B). Collectively, these data show that $\mathrm{L}^{\text {pro }}$, via its catalytic activity, suppresses $S G$ formation.

A
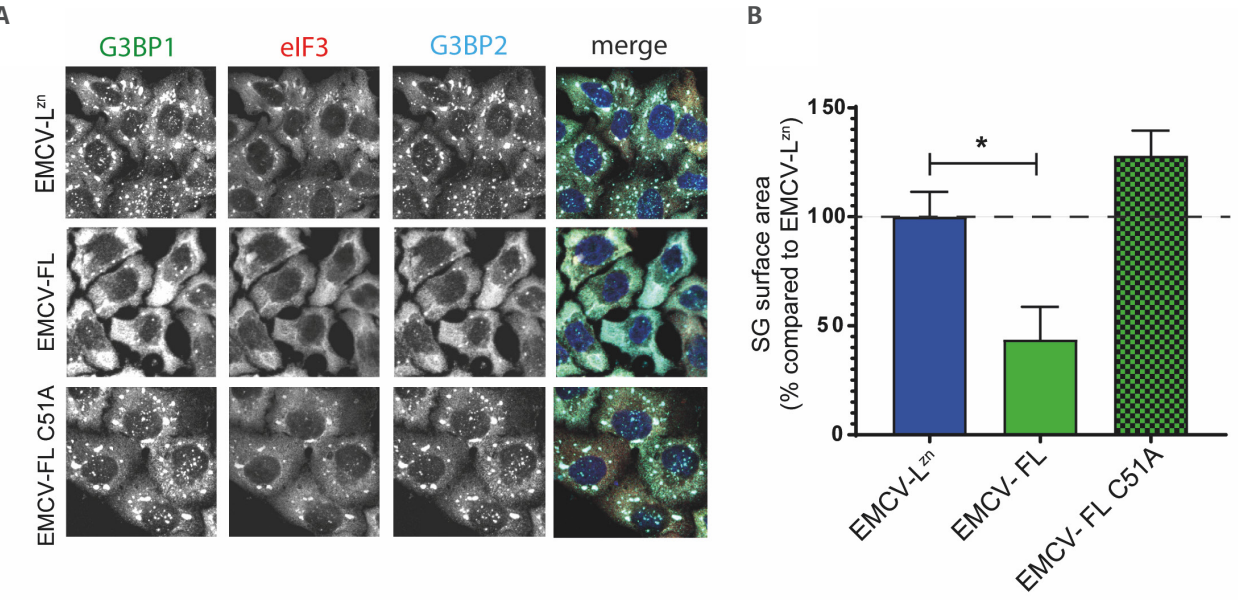

Figure 3: Effect of $L^{\text {pro }}$ on SG formation is dependent on the catalytic activity of $L^{\text {pro }}$. (A) HeLa R19 cells were infected at MOI 10 with chimeric EMCV expressing L Lro (EMCV-FL) or a catalytically inactive L Lro (EMCV-FL C51A) and fixed at 6 hpi. SC formation was visualized by immunofluorescence staining for the $\mathrm{SC}_{\text {markers }} \mathrm{C}_{3} \mathrm{BP}_{1}$ (green), elF3 (red) and $\mathrm{C}_{3} \mathrm{BP}_{2}$ (cyan). (B) Quantification of (A) in which the SC size was analyzed for at least 50 cells per condition. One-way ANOVA with Bonferroni post-hoc test was used to determine statistical significance (" $p<0.05)$

$\mathbf{L}^{\text {pro }}$ prevents SG formation in FMDV-infected cells. To investigate whether $\mathrm{L}^{\text {pro }}$ also suppresses SG formation in aphthovirus-infected cells, we utilized a recombinant FMDV strain in which the leader-coding region was deleted (leaderless virus, LLV)(46). We infected LFPK $\alpha$ v $\beta 6$ cells with wild-type FMDV or LLV and visualized SG formation at 4 hpi. Virus replication was monitored via staining for the capsid protein VP1, while SGs were visualized by anti-G3BP1 staining (Fig 4A). Similar amounts of VP1 were observed in cells infected with wild-type FMDV or LLV, indicating efficient viral replication irrespective of the presence or absence of $\mathrm{L}^{\mathrm{pro}}$, consistent with previous results (46). Infection with wild-type FMDV did not induce SG formation (Fig 4A and Fig 1). In contrast, infection with FMDV LLV triggered SG formation (Fig. $4 \mathrm{~A})$ and, upon quantification, we observed a consistent difference in the number of SG positive cells and the number of SGs per cell between wild-type FMDV-infected cells or LLV-infected cells (Fig 4B). These data demonstrate that FMDV LLV cannot block SG formation. Although we cannot formally exclude the involvement of some RNA element in the deleted region, our data suggests that $\mathrm{L}^{\text {pro }}$ is needed to suppress SG formation during FMDV infection.

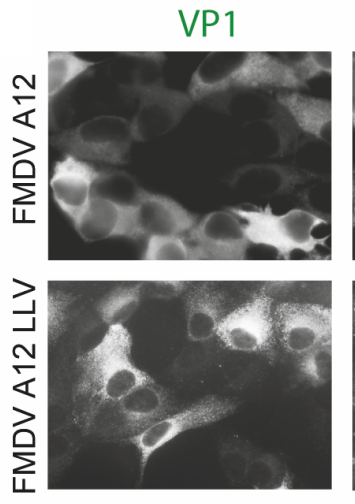

G3BP1
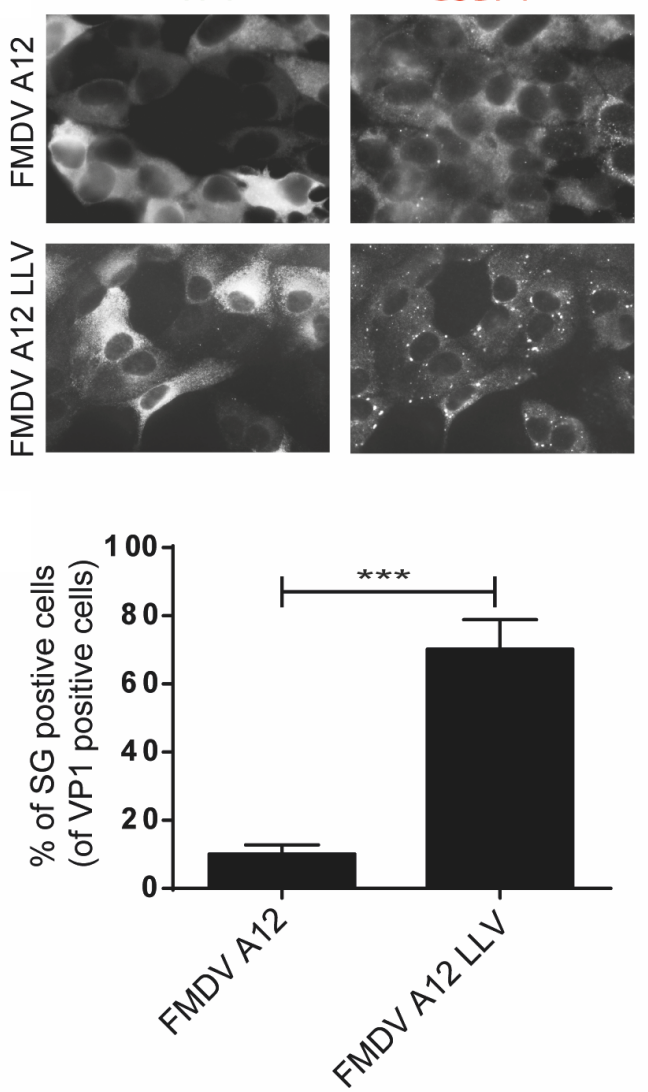

merge
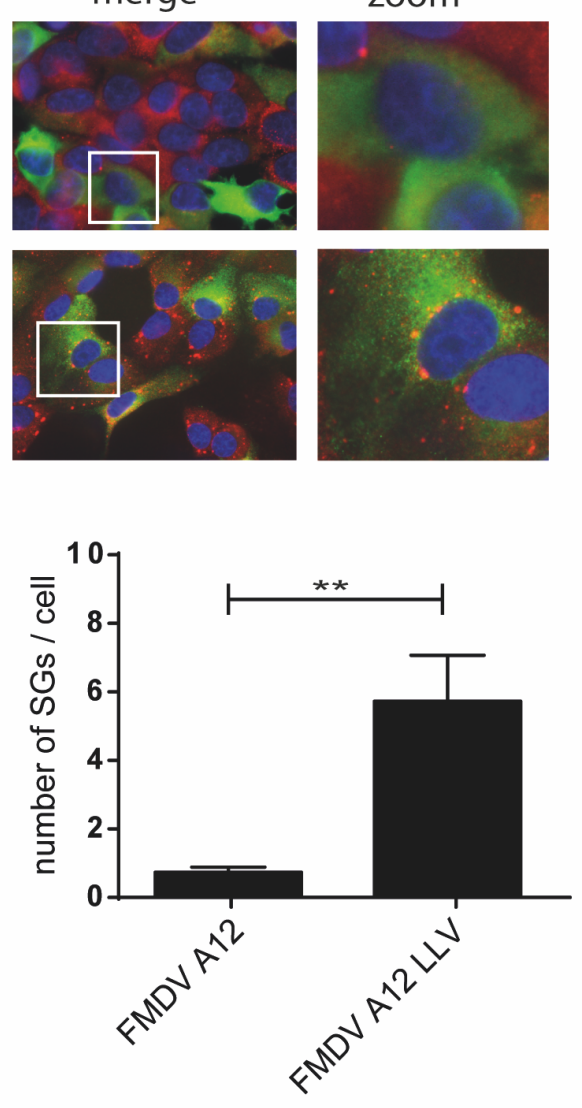

Figure 4: Leaderless FMDV is unable to suppress SG formation. (A) LFPK avB6 cells were infected with FMDV-A12 or leaderless FMDV-A12 (LLV), fixed at 4 hpi and immunofluorescence staining was performed for the viral capsid protein VP1 (green) and the $\mathrm{SC}_{\text {marker }} \mathrm{C}_{3} \mathrm{BP}_{1}$ (red). (B) Quantification of $(\mathrm{A})$ in which the percentage of SC-positive infected cells and the number of SCs per cell were determined in at least 50 cells. One-tailed $t$-test was used to determine statistical significance $(* * 0<0.01, * * * * 0<0.001)$. 
$\mathbf{L}^{\text {pro }}$ does not affect PKR signaling. We investigated whether aphthovirus proteases have an effect upstream of SG assembly, at the level of stress response signaling. To this end, we assessed the phosphorylation of PKR and eIF2 $\alpha$, using flow cytometry. Infection of HeLa cells with EMCV- $\mathrm{L}^{\mathrm{Zn}}$ is known to activate PKR (43), which results in the phosphorylation of eIF2 $\alpha$ and a subsequent halt in translation. Indeed, we observed increased levels of phosphorylated PKR and phosphorylated eIF2 $\alpha$ in EMCV-L $\mathrm{L}^{\mathrm{Zn}}$ infected cells (Fig 5A). The

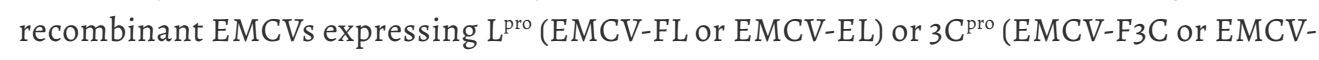
E3C) also failed to suppress PKR or eIF2 $\alpha$ phosphorylation (Fig.5B), indicating that neither $L^{\text {pro }}$ nor $3 C^{\text {pro }}$ affect PKR signaling, at least in HeLa cells infected with chimeric EMCV viruses.

A
p-PKR
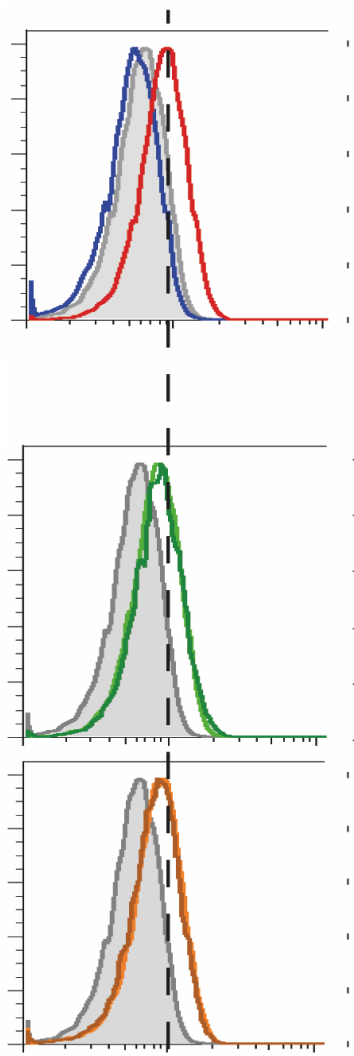

p-elF2a

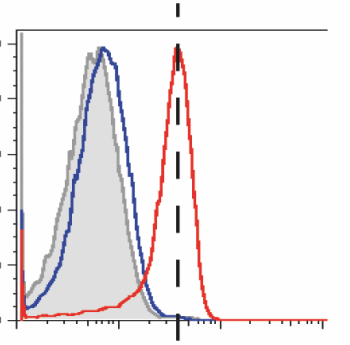

I
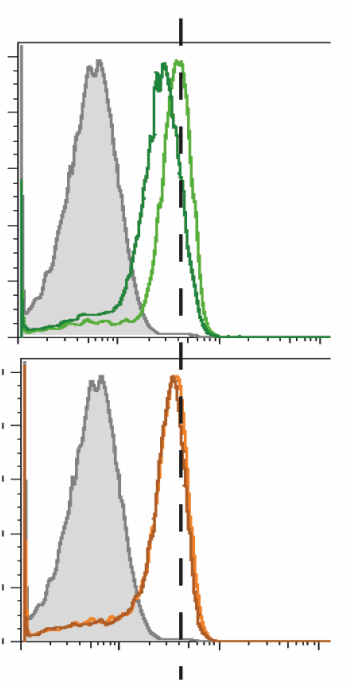

$\square$ EMCV-Lzn

EMCV

non-infected

\section{$\square$ EMCV-E3C \\ EMCV-F3C}

non-infected
Figure 5: L pro does not inhibit PKR signaling. HeLa R19 cells were infected at MOI 10 with EMCV or EMCV-Liz (A) or the indicated chimeric EMCVs (B) and fixed at 6 hpi Subsequent flowcytometry staining was performed for dsRNA and p-PKR or dsRNA and p-elF2 $\alpha$. Craphs represent the level of p-PKR or p-elF2 $\alpha$ in dsRNA positive (infected) cells. Dashed line indicates level of p-PKR or p-elF2 $\alpha$ in EMCV-L ${ }^{2 n}$ infected cells.
SG scaffold proteins are cleaved during aphthovirus infection. Many viruses target SG scaffold proteins to antagonize SG formation. Therefore, we investigated whether SG scaffold proteins are cleaved during FMDV infection. LFPK $\alpha v \beta 6$ cells were infected with wild type FMDV-A12 or A12-LLV and the lysates were subjected to western blot analysis using $\mathrm{G}_{3} \mathrm{BP}_{1}$ antibody. Infection with wild-type FMDV, but not LLV, resulted in the cleavage of $\mathrm{G}_{3} \mathrm{BP} 1$ (Fig $6 \mathrm{~A}$ ). The predicted molecular weight of $\mathrm{G}_{3} \mathrm{BP} 1$ is $52 \mathrm{kDa}$, but we consistently observe the protein migrating at $\sim 70 \mathrm{kDa}$. Upon FMDV infection, we detected two cleavage products that migrate at an apparent molecular weight of $\sim 65 \mathrm{kDa}$ and $\sim 43 \mathrm{kDa}$ (but which thus likely are smaller), but this was not observed in cells infected with FMDV LLV. A $\sim 65$ $\mathrm{kDa}$ cleavage product was first detected at $4 \mathrm{hpi}$ and both cleavage products are clearly visible at 6 hpi. Collectively, our data suggest that $\mathrm{G}_{3} \mathrm{BPI}$ is cleaved at multiple positions during FMDV infection. We also tested the lysates with antibodies against other human SG scaffold proteins, such as TIA-1 and eIF3, but unfortunately these antibodies did not show reactivity with swine proteins.

To investigate whether the ability to cleave SG scaffold proteins is conserved among aphthoviruses, we next studied the integrity of $\mathrm{G}_{3} \mathrm{BP} 1$, as well as G3BP2 and TIA-1, in ERAVinfected cells. To this end, HeLa cells were infected with ERAV or coxsackievirus B3 (CVB3, genus enterovirus), a control virus that is known to cleave $\mathrm{G}_{3} \mathrm{BP} 1$, but not $\mathrm{G}_{3} \mathrm{BP} 2$ or TIA-1 $(24,25,47)$ (Fig 6B). Upon infection with ERAV, cleavages of $\mathrm{G}_{3} B \mathrm{BP}_{1}$ and $\mathrm{G}_{3} \mathrm{BP} 2$, but not TIA1, were observed (Fig. 6B). For $\mathrm{G}_{3} \mathrm{BP} 1$, we observed a large cleavage product that migrated at $\sim 65 \mathrm{kDa}$ and a doublet that ran at $\sim 40 \mathrm{kDa}$. This $\sim 40 \mathrm{kDa}$ doublet was not observed in FMDV-infected cells. Meanwhile we barely detected a $\sim 43 \mathrm{kDa}$ cleavage product in ERAVinfected cells, which we did observe in FMDV-infected cells. Thus, FMDV infection (Fig. 6A) and ERAV infection (Fig. 6B) induced similar - but not identical - cleavages of G3BP1. $\mathrm{G}_{3} \mathrm{BP} 2$, like $\mathrm{G}_{3} \mathrm{BP} 1$, consistently migrated at $\sim 70 \mathrm{kDa}$, but has a predicted molecular weight of only $54 \mathrm{kDa}$. Upon ERAV infection, we observed one G3BP2 cleavage product, running at $\sim 65 \mathrm{kDa}$ (although in reality this product may be smaller).

Next, we investigated whether aphthovirus proteases directly cleave $\mathrm{G}_{3} \mathrm{BP}_{1}$ and $\mathrm{G}_{3} \mathrm{BP} 2$, or indirectly by triggering apoptosis and activation of cellular caspases (Fig 6C). We infected HeLa cells with ERAV and treated them with pan-caspase inhibitor Q-VD-PH (Q-VD) to block caspase activity. While cleavage of PARP, a known caspase substrate, was inhibited by Q-VD (as demonstrated by the reduced amount of PARP cleavage product), ERAV-induced G3BP1 and G3BP2 cleavages were unaffected. Induction of apoptosis, by treatment with actinomycin $\mathrm{D}$ (Act. D), also induced cleavage of $\mathrm{G}_{3} \mathrm{BP} 1$ and $\mathrm{G}_{3} \mathrm{BP} 2$. Importantly, these caspase-mediated $\mathrm{G}_{3} \mathrm{BP} 1$ and $\mathrm{G}_{3} \mathrm{BP} 2$ cleavage products had a different molecular weight than those observed during ERAV infection and were inhibited by addition of Q-VD. Taken together, these results suggest that cleavage of $\mathrm{G}_{3} \mathrm{BP}_{1}$ and $\mathrm{G}_{3} \mathrm{BP}_{2}$ in aphthovirus-infected 
cells is the result of a direct intervention of a viral protease, rather than activation of cellular caspases.

A
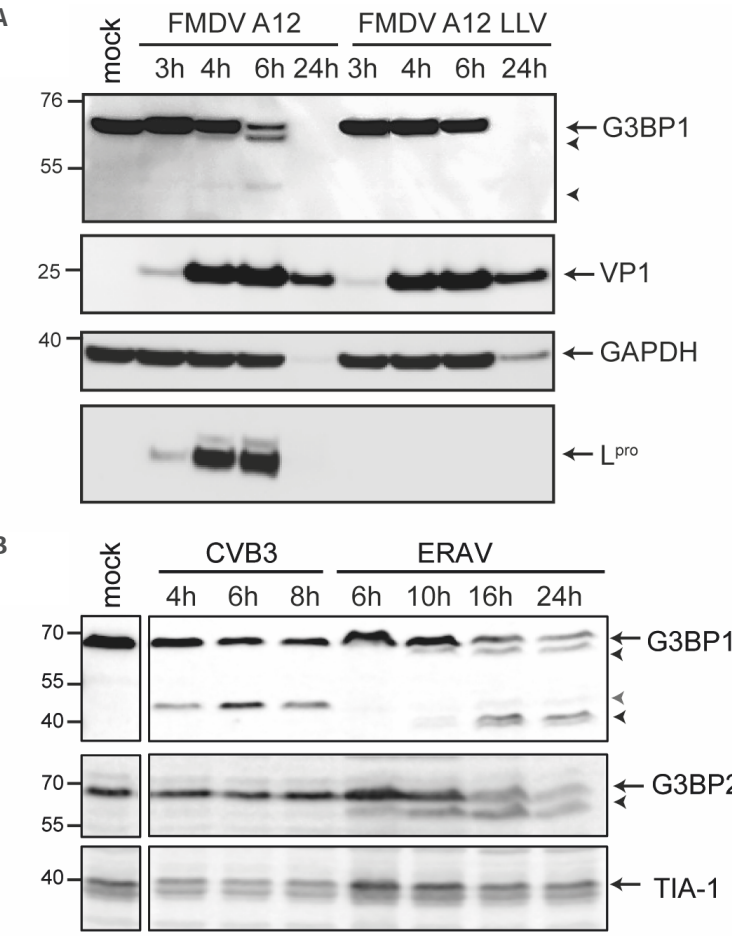

c mock ERAV Act. D

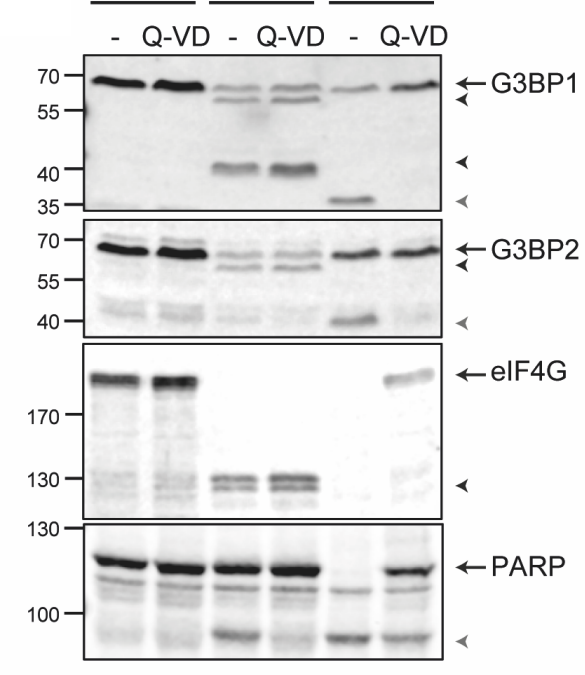

Figure 6: SC scaffold proteins are cleaved during aphthovirus infection. (A) LFPK av $\beta 6$ cells were infected with FMDV-A12 or leaderless FMDV-A12 (LLV) and cells were lysed at indicated times pi. Western blot analysis was performed for $\mathrm{CBP}_{1}, \mathrm{CAPDH}$ and the was perfor viral proteins VP1 and L $\mathrm{L}^{\text {pro. }}$. (B) HeLa R19 cells cells were lysed at indicated times p.i.. Western blot analysis was performed for SC proteins $\mathrm{C}_{3} \mathrm{BP}_{1}, \mathrm{C}_{3} \mathrm{BP} 2$ and TIA-1. (C) HeLa R19 cells were infected with ERAV at $\mathrm{MOI} 10$ or treated with 5 $\mathrm{ug} / \mathrm{ml}$ Actinomycin D. Subsequently cells were incubated for 16 hrs in thequenty cells were incubated for 10 his of $10 \mu \mathrm{MQVD}$. Cell lysates were subjected to western blot analysis for $\mathrm{C}_{3} \mathrm{BP}_{1}$ and $\mathrm{C}_{3} \mathrm{BP}_{2}$, translation initiation factor $4 \mathrm{C}(\mathrm{elF} 4 \mathrm{C})$ and PARP. Arrows indicate full length proteins, black arrowheads indicate aphthovirusinduced cleavage products, grey arrowheads indicate cleavage products induced upon CVB3 infection (B) or cleavage products induced upon the induction of apoptosis (C). were infected at MOI 10 with CVB3 or ERAV and
$\mathbf{L}^{\text {pro }}$ cleaves $\mathrm{G}_{3} \mathbf{B P} \mathbf{1}$ and $\mathbf{G}_{3} \mathbf{B P 2}$. It has been suggested that EMCV infection triggers $\mathrm{G}_{3} \mathrm{BP} \mathbf{1}$ cleavage via $3 C^{\text {pro }}(28)$. This would prevent us from investigating whether aphthovirus $L^{\text {pro }}$ cleaves $\mathrm{G}_{3} \mathrm{BP} 1$ and $\mathrm{G}_{3} \mathrm{BP} 2$ by infecting cells with EMCV-FL and EMCV-EL. However, the cleavage of G3BP1 by EMCV is debated and could not be reproduced in another study (26). As such, we first infected HeLa cells with EMCV, EMCV- $\mathrm{L}^{\mathrm{Zn}}$ or CVB3 - as a positive control - and determined the integrity of $\mathrm{G}_{3} \mathrm{BP}_{1}$ and $\mathrm{G}_{3} \mathrm{BP} 2$ via western blot analysis (Fig. $7 \mathrm{~A}$ ). We did not detect cleavage of G3BP1 upon EMCV infection, using two different antibodies that recognize different epitopes within G3BP1. Our observations are consistent with reports that EMCV suppresses SG formation via its leader protein $(9,26)$.

To investigate which aphthovirus protease cleaves SG scaffold proteins, we infected HeLa cells with the EMCVs expressing $\mathrm{L}^{\text {pro }}$ and $3 \mathrm{C}^{\text {pro }}$ (EMCV-FL, EMCV-EL, EMCV-F3C and EMCV $\mathrm{E}_{3} \mathrm{C}$ ) and performed western blot analysis for $\mathrm{G}_{3} \mathrm{BP} 1$ and $\mathrm{G}_{3} \mathrm{BP} 2$. To compare the migration patterns of the cleavage products to those observed during aphthovirus infection, a lysate of ERAV-infected cells was run on the same gel (Fig. 7B). In agreement with the observation that leaderless FMDV failed to cleave G3BPI (Fig. 6A), we observed that infection with EMCV expressing $\mathrm{L}^{\text {pro }}$ (EMCV-FL and EMCV-EL) resulted in the cleavage of $\mathrm{G}_{3} \mathrm{BP}_{1}$ and $\mathrm{G}_{3} \mathrm{BP} 2$ (Fig. $8 \mathrm{~A})$. A G3BP1 cleavage product migrating at $\sim 65 \mathrm{kDa}$ was observed during infection with EMCV-FL or EMCV-EL. A similar sized cleavage product prominently appeared during FMDV and ERAV infection. A $\sim 33 \mathrm{kDa}$ G3BPI cleavage product, similar to the product that accumulated in cells infected with FMDV, also accumulated in cells infected with EMCVFL. Such a $\sim 43 \mathrm{kDa}$ product was barely detected in ERAV-infected cells and did not appear in cells infected with EMCV-EL. Instead, a doublet cleavage product migrating at $\sim 40 \mathrm{kDa}$ was observed in ERAV-infected cells. We observed a similar doublet in cells infected with EMCV-F3C and EMCV-E3C, suggesting that $3 \mathrm{C}^{\text {pro }}$ can also cleave $\mathrm{G}_{3} \mathrm{BP}$. Intriguingly, such $\mathrm{a} \sim 40 \mathrm{kDa}$ doublet was not observed in FMDV-infected cells. $3 \mathrm{C}^{\text {pro }}$ can also cleave $\mathrm{G}_{3} \mathrm{BP}$ albeit inefficiently and this had no effect on the integrity of SGs (Fig. 2). In ERAV-infected cells, we detected a G3BP2 cleavage product running at $\sim 65 \mathrm{kDa}$ (Fig. 6B and Fig. 7B). We observed a similar G3BP2 cleavage product, running at $\sim 65 \mathrm{kDa}$, upon infections with EMCV-FL and EMCV-EL (Fig 7B), suggesting that $L^{\text {pro }}$ cleaves G3BP2. Notably, infection with EMCV-FL resulted in a number of other bands that showed reactivity with the G3BP2 antibody, ranging from $\sim 55 \mathrm{kDa}$ to $\sim 45 \mathrm{kDa}$. In particular, infection with EMCV-FL and EMCV-EL induced a product of $\sim 45 \mathrm{kDa}$ (indicated with *), that was not observed during ERAV infection. We did not observe cleavage of G3BP2 upon infection with either EMCV$\mathrm{F}_{3} \mathrm{C}$ or $\mathrm{EMCV}-\mathrm{E}_{3} \mathrm{C}$. Together, these data suggest that $\mathrm{L}^{\text {pro }}$ has a major impact on the integrity of $\mathrm{G}_{3} \mathrm{BP}_{1}$ and $\mathrm{G}_{3} \mathrm{BP}_{2}$ in infected cells. 
To gain more insight into the timing of $\mathrm{G}_{3} \mathrm{BP} 1$ and $\mathrm{G}_{3} \mathrm{BP} 2$ cleavage by $\mathrm{L}^{\text {pro }}$, we next infected HeLa cells with EMCV-FL for different durations and performed western blot analysis of G3BP1 and G3BP2 (Fig 7C). The $\sim 65 \mathrm{kDa}$ G3BP1 cleavage product can be observed at 6 and 8 hpi. Meanwhile, we observed the $\sim 43 \mathrm{kDa}$ fragment at $8 \mathrm{hpi}$, suggesting that the first cleavage event allows the second cleavage event to occur. This sequential cleavage was also seen in FMDV-infected cells (Fig. 6A). A G3BP2 cleavage product, running at $\sim 65 \mathrm{kDa}$, was first observed at 6 hpi but was more clearly visible at 8 hpi (Fig $7 \mathrm{C}$ ). As anticipated, infection with an EMCV encoding the catalytically inactive FMDV L $^{\text {pro }}$ (EMCV-FL C51A) failed to cleave $\mathrm{G}_{3} \mathrm{BP} 1$ and $\mathrm{G}_{3} \mathrm{BP} 2$ (Fig $7 \mathrm{C}$ ). Collectively, our observations strongly suggest that $\mathrm{L}^{\text {pro }}$ is responsible for cleaving SG scaffold proteins in aphthovirus-infected cells.

To determine the location of the $\mathrm{L}^{\text {pro }}$ and $3 \mathrm{C}^{\text {pro }}$ cleavage sites in $\mathrm{G}_{3} \mathrm{BP}$, we co-expressed $\mathrm{N}$-terminally Flag-tagged $\mathrm{G}_{3} \mathrm{BP} 1$ with EGFP-tagged $\mathrm{L}^{\text {pro }}$ or $3 \mathrm{C}^{\text {pro }}$ (Fig $7 \mathrm{D}$ ). Under these conditions, we observed cleavage patterns of G3BP1 that resembled those observed in FMDV- and ERAV-infected cells and upon infection with the recombinant EMCVs. As the Flag-tag is located at the $\mathrm{N}$-terminus of $\mathrm{G}_{3} \mathrm{BP} 1$, it can be concluded that the $\mathrm{L}^{\text {pro }}$ cleavage site yielding a $\sim 65 \mathrm{kDa}$ product is located in the $\mathrm{C}$-terminal part of $\mathrm{G} 3 \mathrm{BP} 1$. A second FMDV $\mathrm{L}^{\text {pro }}$ cleavage site, yielding a $\sim 43 \mathrm{kDa}$ fragment - and also the $3 \mathrm{C}^{\text {pro }}$ cleavage sites, yielding a $\sim 40$ $\mathrm{kDa}$ doublet - are located close to the middle of the protein, but slightly more towards the C-terminus. We also co-expressed Flag-tagged G3BP2 with EGFP-tagged L L $^{\text {pro }}$ (Fig 7E). We observed G3BP2 cleavages that were highly similar to those observed upon infection with ERAV, EMCV-FL and EMCV-EL. This suggests that the major L $^{\text {pro }}$ cleavage site in $\mathrm{G}_{3} \mathrm{BP} 2$ is located in the C-terminal part of the protein. Notably, we did not observe the extra $\sim 45 \mathrm{kDa}$ cleavage product that was apparent upon infection with EMCV-FL or EMCV-EL, supporting the idea that this product is an artefact. We observed multiple cleavage products migrating around $\sim 55 \mathrm{kDa}$ upon overexpression of FMDV $\mathrm{L}^{\text {pro }}$, similar as in cells infected with EMCVFL. These products were not observed upon infection with EMCV-EL (Fig. 7B) and also not upon overexpression of ERAV $L^{\text {pro }}$. The approximate $L^{\text {pro }}$ and $3 \mathrm{C}^{\text {pro }}$ cleavage sites in $\mathrm{G}_{3} \mathrm{BP} 1$ and $\mathrm{G}_{3} \mathrm{BP}_{2}$ - based on the apparent molecular weights of the cleavage products are schematically represented in Fig. 7F. Collectively, these results indicate that the major cleavage products of $\mathrm{G}_{3} \mathrm{BP} 1$ and $\mathrm{G} 3 \mathrm{BP} 2$, migrating at $\sim 65 \mathrm{kDa}$, are generated upon cleavage of the $\mathrm{C}$-terminus of these proteins. The additional G3BP1 and $\mathrm{G}_{3} \mathrm{BP} 2$ cleavage products produced by FMDV L $\mathrm{L}^{\text {pro }}$ and migrating at $\sim 43 \mathrm{kDa}$ and around $\sim 55 \mathrm{kDa}$, respectively - are generated upon cleavage in the $\mathrm{C}$-terminal half of the protein, close to the middle of the protein. The cleavage sequences of some substrates of $\mathrm{L}^{\text {pro }}$ are known, but we were not successful in identifying the cleavage sites in $\mathrm{G}_{3} \mathrm{BP} 1$ and $\mathrm{G}_{3} \mathrm{BP} 2$. Nevertheless, the cleavage sites of aphthovirus $\mathrm{L}^{\text {pro }}$ in $\mathrm{G}_{3} \mathrm{BP}$ i clearly differ from that of enterovirus $3 \mathrm{C}^{\text {pro }}$ (Fig $\left.6 \mathrm{~B}\right)$.

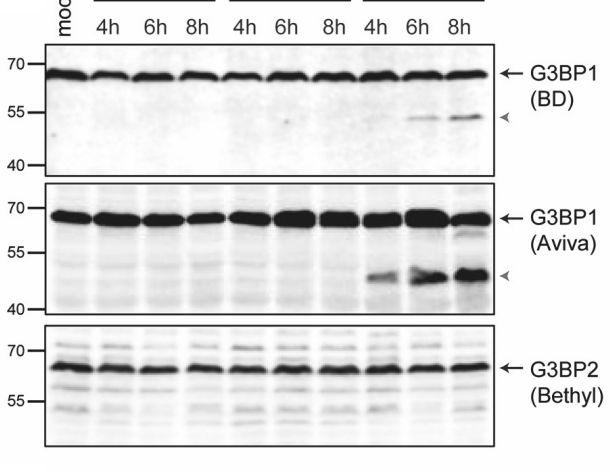

B
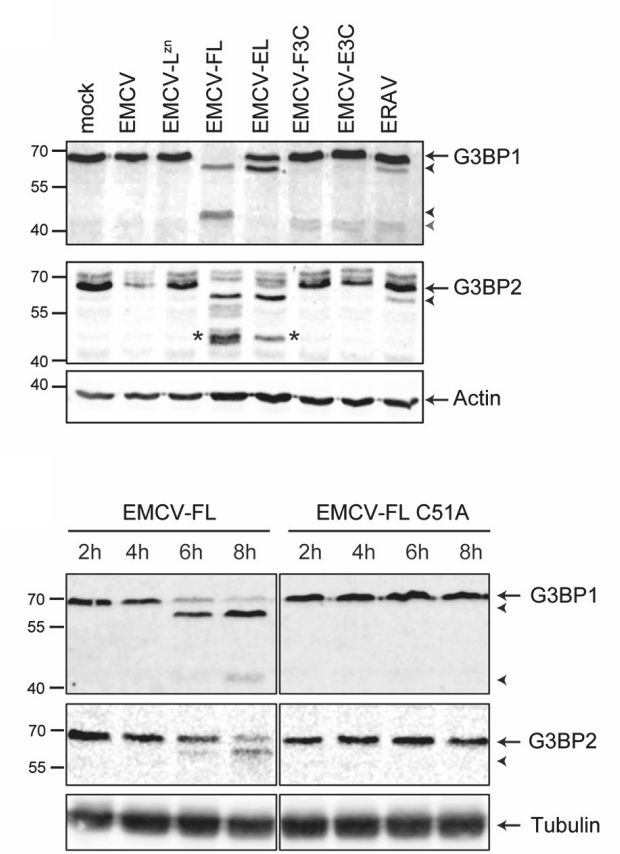

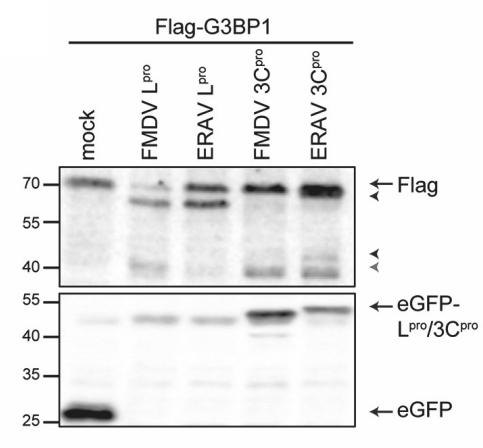

E
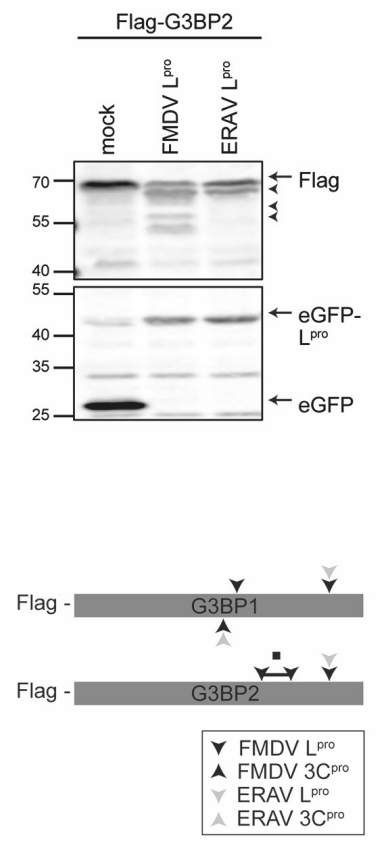

Figure 7: Aphthovirus L $\mathrm{L}^{\text {pro }}$ and $3 \mathrm{C}^{\text {pro }}$ cleave SG proteins. (A) HeLa R19 cells were infected at MOI 10 with EMCV, EMCV-L or $\mathrm{CVB}_{3}$ and cells were lysed at indicated times p.i. Western blot analysis was performed with two different antibodies against $\mathrm{C}_{3} \mathrm{BP}_{1}$ (recognizing differented (E) or ERAV and cells were lysed at $8 \mathrm{hrs}$. (ECMVs) or $10 \mathrm{hpi} \mathrm{(ERAV).} \mathrm{Subsequent} \mathrm{western} \mathrm{blot} \mathrm{analysis}$ was performed for $\mathrm{C}_{3} \mathrm{BP}_{1}$ and $\mathrm{C}_{3} \mathrm{BP}_{2}$. " indicates additional (artificial) $\mathrm{C}_{3} \mathrm{BP} 2$ cleavage product (C) HeLa R19 cells were infected at MOI 10 with EMCV-FL or EMCV-FL C51A and cells were lysed at the indicated timepoints. Subsequent western blot analysis was performed for $\mathrm{C}_{3} \mathrm{BP}_{1}$ and $\mathrm{C}_{3} \mathrm{BP} 2$. ( D\&E) Hek293T cells were co-transfected with a Flag-tagged $\mathrm{C}_{3} \mathrm{BP} 1$ or $\mathrm{C}_{3} \mathrm{BP}_{2}$ and a ECFP-tagged viral protease as indicated. $16 \mathrm{hrs}$. post transfection, cells were lysed and subjected to western blot analysis for FLAG and ECFP. Arrows indicate full length proteins, black arrowheads indicate L Lro-induced cleavage products, grey arrowheads indicate $3 \mathrm{C}^{\mathrm{Pro}-}$-induced cleavage products. (F) Schematic representation of approximate cleavage sites, based on apparent molecular weight of the cleavage products, in $\mathrm{C}_{3} \mathrm{BP}_{1}$ and $\mathrm{C}_{3} \mathrm{BP} 2$ for the different aphthovirus proteases. A region in $\mathrm{C}_{3} \mathrm{BP}_{2}$ containing multiple FMDV $L^{\text {pro }}$ cleavage sites is indicated by . 


\section{Discussion}

Over recent years, SGs are increasingly seen as important antiviral signaling platforms (2, 4-7). Several molecules involved in RLR signaling and stress response signaling have been found to localize in SGs $(5,8-10)$. Many viruses interfere with stress responses and the formation of SGs. Several picornaviruses belonging to the Enterovirus and Cardiovirus genus have been shown to actively suppress the stress response pathway $(9,24-26)$, but limited knowledge was available for members of the Aphthovirus genus. Here, we provide evidence that aphthoviruses suppress SG formation through $\mathrm{L}^{\mathrm{pro}}$. This papain-like protease is well known for its ability to cleave translation initiation factor eIF4G and thereby shuts off host translation (29). We showed that SG formation is suppressed in cells infected with wildtype FMDV but not in a leaderless mutant FMDV. Heterologous expression of $\mathrm{L}^{\text {pro }}$ of FMDV or ERAV in a mutant EMCV lacking its own leader protein, also suppressed SG formation. $L^{\text {pro }}$ did not affect PKR activation and eIF2 $\alpha$ phosphorylation. Instead, western blot analysis showed that SG scaffold proteins $\mathrm{G}_{3} \mathrm{BP} 1$ and $\mathrm{G}_{3} \mathrm{BP} 2$ were cleaved in aphthovirus-infected cells. Similar cleavages were observed in cells infected with mutant EMCV expressing either $\mathrm{L}^{\text {pro }}$ and upon co-expression of Flag-tagged $\mathrm{G}_{3} \mathrm{BP} 1 / 2$ with $\mathrm{L}^{\text {pro }}$. Collectively, our data demonstrate an essential role of $\mathrm{L}^{\text {pro }}$ in suppressing $\mathrm{SG}$ formation in aphthovirus-infected

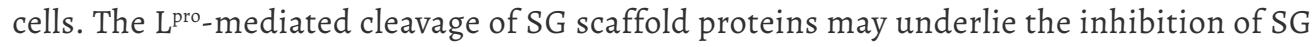
formation, although it cannot be excluded that a yet unidentified cleavage of other cellular protein(s) involved in stress signaling or SG formation also contributes to this inhibition. Besides dsRNA-dependent and PKR-mediated SG formation, also SG formation induced by sodium arsenite, which induces stress via a ROS-dependent mechanism via a different kinase (HRI), was inhibited. This lends support to the idea that $\mathrm{L}^{\text {pro }}$ has an inhibitory effect on formation and/or stability of SGs by cleaving essential SG components.

Cleavage of SG components likely also underlies the inhibition of SG formation by enteroviruses. Enterovirus infection results in formation of small SGs, which disappear as infection progresses. This observation has been attributed to cleavage of $\mathrm{G}_{3} \mathrm{BP} 1$ by enterovirus $3 \mathrm{C}^{\mathrm{pro}}(24)$. However, recently evidence was presented that enterovirus $2 \mathrm{~A}^{\text {pro }}$ rather than $3 \mathrm{C}^{\text {pro }}$ is responsible for suppression of $\mathrm{SG}$ formation in enterovirus-infected cells (48). Expression of $2 \mathrm{~A}^{\text {pro }}$, but not $3 \mathrm{C}^{\text {pro }}$, of several enteroviruses was shown to inhibit SG formation induced by sodium arsenite or heat shock. Furthermore, infection of a recombinant EV-A71, in which $2 \mathrm{~A}^{\text {pro }}$ activity was inactivated, led to the formation of typical SGs. The mechanism by which $2 \mathrm{~A}^{\text {pro }}$ suppresses SG formation has not been elucidated, but these findings challenge the importance of cleavage of $\mathrm{G}_{3} \mathrm{BP} 1$ as an antagonizing mechanism to inhibit SG formation. Enterovirus $3 C^{\text {pro }}$ does not cleave G3BP2 (25). Possibly the simultaneous cleavage of both $\mathrm{G}_{3} \mathrm{BP} 1$ and $\mathrm{G}_{3} \mathrm{BP} 2$ by $\mathrm{L}^{\text {pro }}$ is required to suppress $\mathrm{SG}$ formation. In line with this, it has been shown that $S G$ formation is blocked in cells in which $\mathrm{G}_{3} \mathrm{BP}_{1}$ and $\mathrm{G}_{3} \mathrm{BP}_{2}$ are depleted, but not in cells lacking only one of the G3BPs (49). Alternatively, the G3BP1 cleavage product generated by $\mathrm{L}^{\text {pro, }}$, which differs from that of enterovirus $3 \mathrm{C}^{\text {pro }}$, may have a dominant negative effect on $\mathrm{SG}$ formation. The exact mechanisms used by aphthovirus $\mathrm{L}^{\text {pro }}$ and enterovirus $2 \mathrm{~A}^{\text {pro }}$, both of which are considered as "security proteins" (50), to suppress SG formation remains to be elucidated.

We found a strongly reduced amount of G3BP1 containing SGs in cells infected with wildtype FMDV. This observation is in agreement with previous observations that some small SGs that contain TIA-1 and Sam68, but lack G3BP1, are formed in FMDV-infected cells $(51,52)$. These small SGs resemble the small "atypical" SGs observed in cells expressing enterovirus $2 \mathrm{~A}^{\mathrm{pro}}(25,48)$. These atypical SGs consist of cellular mRNAs of which translation is halted due to the cleavage of eIF4G by $2 \mathrm{~A}^{\text {pro }}(25,48)$. There is conflicting literature about the composition of these atypical SGs. Some studies have shown that these atypical SGs contain specific SG markers, i.e. TIA-1, TIAR and Sam68, but lack other SG markers, G3BPs and eIFs $(48,53)$, whereas others have shown that G3BP1-containing SGs are formed during enterovirus infection $(24,54)$ or upon overexpression of enterovirus $2 \mathrm{~A}^{\text {pro }}(25)$. Upon infection with recombinant EMCV expressing either FMDV L ${ }^{\text {pro }}$ or ERAV L ${ }^{\text {pro }}$, we observed small SGs containing TIA-1, G3BPs and eIF3. Although it is unclear what the exact composition is of atypical SGs, it seems plausible that enterovirus $2 \mathrm{~A}^{\text {pro }}$ and aphthovirus $\mathrm{L}^{\text {pro }}$ can induce the formation of atypical SGs via their shared ability to inhibit host cell translation.

SG formation is induced upon the activation of eIF2 $\alpha$ kinases such as PKR and PERK. While enterovirus infection induces PKR activation, leading to an increase in both p-PKR and p-eIF2 $\alpha$ levels $(55,56)$, FMDV infection was shown to block activation of this pathway (57),

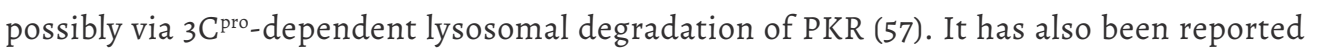
that the titers of FMDV LLV can be rescued in PKR knock-out cells (58), hinting to a possible role for $\mathrm{L}^{\text {pro }}$ in suppressing PKR signaling. We failed to observe any inhibitory effect on PKR signaling in cells infected with recombinant EMCV expressing either $\mathrm{L}^{\text {pro }}$ or $3 \mathrm{C}^{\text {pro }}$. This may be a limit of our experimental setup to express these proteases outside the context of aphthovirus infection; e.g. it may suggest that antagonizing PKR signaling requires the action of multiple aphthovirus proteins. Nevertheless, we observed a clear inhibitory effect of $L^{\text {pro }}$ on SG formation in cells infected with the chimeric EMCV-Lpro, independent of any effect on PKR signaling. Obviously, this does not rule out the possibility that effects of $\mathrm{L}^{\text {pro }}$ and/or $3 \mathrm{C}^{\text {pro }}$ on PKR signaling also contribute to the inhibition of $\mathrm{SG}$ formation in aphthovirus-infected cells, where this inhibition seems to be more pronounced than in cells infected with EMCV-L $\mathrm{L}^{\text {pro }}$. Multiple complementary SG inhibitory mechanisms may coexist in aphthovirus-infected cells, one of which in mediated by FMDV L ${ }^{\text {pro }}$. 
Recently, G3BPI was reported to bind to the IRES of FMDV and function as a translation inhibitor, and it was shown that $F M D V{ }_{3} \mathrm{C}^{\text {pro }}$ induced the cleavage of $\mathrm{G}_{3} \mathrm{BP}_{1}$ (59). Interestingly both the $\mathrm{N}$-terminal and $\mathrm{C}$-terminal $\mathrm{G} 3 \mathrm{BP} 1$ cleavage product maintained the negative effect on translation (59). In our study, we observed that FMDV and ERAV $3 C^{\text {pro }}$ can cleave G3BP1 to some extent when expressed through recombinant EMCV. While this cleavage did not affect SG formation, it might thus affect viral translation.

In this study, we used a recombinant EMCV lacking its stress antagonist, the leader protein, to express heterologous proteinases of FMDV and ERAV. It has been suggested by others

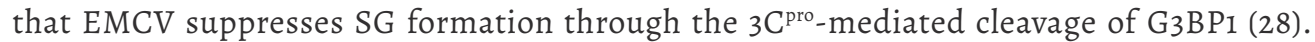
As reported previously for both EMCV and $\operatorname{TMEV}(9,26)$, we show that leader-inactivated EMCV (EMCV-L $\mathrm{L}^{\mathrm{Zn}}$ ) failed to suppress SG formation, whereas no SGs are observed in cells infected with wildtype EMCV. The leader of EMCV lacks proteolytic activity and no cleavage of either $\mathrm{G}_{3} \mathrm{BP}_{1}$ or $\mathrm{G}_{3} \mathrm{BP}_{2}$ was observed in EMCV infected cells. Thus, our data challenge the suggestion that $\mathrm{EMCV} 3 \mathrm{C}^{\text {pro }}$ suppresses $\mathrm{SG}$ formation by cleaving $\mathrm{G}_{3} \mathrm{BP}$.

In conclusion, in this study we present evidence that aphthoviruses actively suppress SG formation through the proteolytic activity of $\mathrm{L}^{\text {pro }}$, and we identified two SG scaffold proteins, $\mathrm{G}_{3} \mathrm{BP} 1$ and $\mathrm{G}_{3} \mathrm{BP} 2$, as new targets of $\mathrm{L}^{\mathrm{pro}}$. These results add aphthoviruses to a growing list of viruses that prevent $\mathrm{SG}$ assembly to antagonize the stress response. These viruses include several flaviviruses (e.g. Hepatitis C virus, Dengue virus and West Nile virus), which use viral subgenomic RNA or nonstructural proteins to sequester SG proteins such as G3BP1, TIA-1 and TIAR to prevent SG formation $(13,14)$, as well as alphaviruses such as Semliki-forest virus (SFV), which prevents SG formation by sequestration of G3BP1 and $\mathrm{G} 3 \mathrm{BP} 2$ by its nsP3 protein (15-17). Although these examples all hint to an important antiviral role of SGs, their exact role as a platform for antiviral signaling is yet poorly understood and more studies are warranted to potentially develop new tools to fight virus infection.

\section{Materials and Methods}

Cells and viruses. HeLa R19, BHK21 and Hek293T cells were maintained in Dulbecco's Modified Eagle's Medium (DMEM) supplemented with 10\% FCS (V/V). LFPK $\alpha$ v $\beta 6$ cells (40) were obtained from the Foreign Animal Disease Diagnostic Laboratory (FADDL) at the PIADC. These cells were maintained in minimal essential medium (MEM) supplemented with $10 \%$ FCS (V/V) and 1\% antibiotics and non-essential amino acids. BHK21 cells used for FMDV propagation were maintained in MEM supplemented with 10\% FCS (V/V), 10\% tryptose phosphate broth, $1 \%$ antibiotics and non-essential amino acids. FMDV A12-WT (wild type) was generated from the full-length serotype A12 infectious clone, PRMC35
(60) and A12-LLV2 (leaderless virus) was derived from the infectious clone lacking the Lb coding region, PRM-LLV2 (46). Viruses were propagated in BHK-21 and concentrated by polyethylene glycol precipitation, titrated on BHK-21 cells, and stored at $-70^{\circ} \mathrm{C}$. ERAV (NM-11/67 strain) (gift from D. Rowlands and T. Tuthill (University of Leeds, Leeds, United Kingdom) (61)) was obtained after passage on HeLa R19 cells and subsequently concentrated by ultracentrifugation through a $30 \%$ sucrose cushion at $140,000 \mathrm{xg}$ for 16 hours in a SW $32 \mathrm{Ti}$ rotor and stored at $-80^{\circ} \mathrm{C}$. Recombinant EMCVs were generated by cloning the genes of interest into the XhoI/NotI restriction sites from the PM16.1-VFETQG-Zn infectious clone that was described previously (43), the Strep2 tag was omitted in the viruses used in this study. Viruses were recovered by transfection of run-off RNA transcripts into BHK-21 cells. Upon total CPE, viruses were concentrated by ultracentrifugation (as described for ERAV) and stored at $-80^{\circ} \mathrm{C}$.

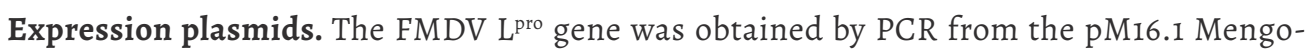
FL infectious clone (45) using the following oligonucleotides 5'-CTCGAGCTGACACTGTACA ACGGTGAG-3' and '5'-GCGGCCGCTTTGAGCTTGCGTTGAACCTTG-3', the FMDV $3 \mathrm{C}^{\text {pro }}$ gene was obtained by PCR using the following oligonucleotides 5'-AAAAAC TCGAGAGTGGTGCCCCACCG-3' and 5'-AAAAGCGGCCGCCTCGTGGTGTGGTTCGGG-3' and the pBind-3C-VP16 FMDV plasmid (62) as template. The ERAV $\mathrm{L}^{\text {pro }}$ and $3 \mathrm{C}^{\text {pro }}$ gene were obtained by PCR from ERAV viral RNA (NM-11/67 strain) using the following oligonucleotides 5'-CTCGAGATGGACAAATTCTTGCAAAAGAAAAC-3' and 5'-GCGGCCGCCTTCTCATACCTCTGATGTAAC-3' for $\mathrm{L}^{\text {pro }}$, for $3 \mathrm{C}^{\text {pro }}$ the following oligonucleotides were used 5'AAAACTCGAGACTGGTGTGCCAGCAACTG-3' and 5'AAAAAGCGGCCGCCTGTTTCTGAGGGAGAGTTCGC- 3 '. The oligonucleotides encode flanking XhoI and NotI restriction sites that were used to ligate the PCR products into the desired plasmids. The $3 \mathrm{C}^{\text {pro }}$ genes were ligated into the pcDNA-GFP vector described previously (11). The $\mathrm{L}^{\text {pro }}$ genes were ligated into the pIRES-EGFP-MCS plasmid. This is an pIRES2-EGFP based vector, in which the multiple cloning site has been relocated after the EGFP, allowing expression of an $\mathrm{N}$-terminal EGFP fusion protein under expression of an EMCV IRES. The plasmids encoding Flag-G3BP1 and Flag-G3BP2 have been described elsewhere (11).

Antibodies. The following antibodies were used for IFA staining procedures: FMDV VP1 (mouse monoclonal Ab 6HC4), $\alpha$ dsRNA (J2, English\&Scientific consulting), $\alpha \mathrm{G}_{3} \mathrm{BP}_{1}$ (clone 23/G3BP, BD biosciences), $\alpha$ G3BP1 (ARP37713, Aviva systems biology), $\alpha G_{3} B P 2$ (A302-040A, Bethyl laboratories), $\alpha e I F 3$ (N-20, SantaCruz) and $\alpha$ TIA-1 (C-20, SantaCruz). Alexa Fluor 488, Alexa Fluor 594 and Alexa Fluor 647 conjugated secondary antibodies (Molecular Probes, Invitrogen) were used for detection. For flowcytometry staining we used the adsRNA (J2, English\&Scientific consulting), $\alpha$ p-PKR (E120, Abcam), $\alpha$ p-eIF2 $\alpha$ (E90, Abcam) antibodies 
and Alexa488 or Alexa647 conjugated (Molecular Probes, Invitrogen) secondary antibodies. For western blot we used the antibodies described above against $\mathrm{G}_{3} \mathrm{BP}_{1}, \mathrm{G}_{3} \mathrm{BP} 2$ and TIA1 and the additional antibodies: $\alpha$ PARP (\#11835238001; Roche Diagnostics), $\alpha$ Flag (M2, Sigma), aGFP (OSE00003G, Invitrogen), atubulin (DMiA, Sigma). Respective IRdye680 or IRdye800 conjugated secondary antibodies (LiCOR) were used for detection.

Immune Fluorescence Analysis (IFA). LF-PK $\alpha v \beta 6$ cells were grown on $12 \mathrm{~mm}$ glass coverslips and infected with FMDV WT or LLV (MOI 10) for $1 \mathrm{~h}$ at $37^{\circ} \mathrm{C}$. After adsorption, cells were rinsed with acidic buffer $(150 \mathrm{mM} \mathrm{NaCl}$ in $20 \mathrm{mM}$ morpholineethane-sulfonic acid (MES) $\mathrm{pH}$ 6.0), before adding MEM and proceeding with the incubation at $37^{\circ} \mathrm{C}$ for the indicated durations. The cells were fixed in $4 \%$ paraformaldehyde, permeabilized with $0.5 \%$ Triton $\mathrm{X}-100^{\circledast}$ (Sigma) in PBS, blocked with blocking buffer (PBS, $2 \%$ bovine serum albumin $[\mathrm{BSA}], 5 \%$ normal goat serum, $10 \mathrm{mM}$ glycine) and then incubated overnight at $4^{\circ} \mathrm{C}$ with primary antibodies against FMDV VP1 (mouse monoclonal $\mathrm{Ab} 6 \mathrm{HC}_{4}$ ) and $\mathrm{G}_{3} \mathrm{BP} 2$ (A302-040A-Bethyl Laboratories, Inc, Montgomery, TX). Alexa Fluor 488 and Alexa Fluor 594 (Molecular Probes, Invitrogen) conjugated secondary Abs were used for detection of green and red signal respectively. Nuclei were visualized by DAPI staining included in ProLong Gold Antifade mounting media (Invitrogen). Cells were examined in a fluorescence microscope and the images were taken with Nikon DS-Qi1 digital camera and NIS-Elements Advance Research v3.00 software (Nikon Instruments, Inc, Melville, NY). Alternatively, HeLa R19 cells were grown on $12 \mathrm{~mm}$ glass coverslips and the next day infected with the indicated viruses (MOI 10). At the indicated time points cells were washed in PBS before being fixed in $4 \%$ paraformaldehyde for $30 \mathrm{~min}$. Residual paraformaldehyde was washed away using PBS+10 mM glycine. Cells were permeabilized in PBS+0.1\% Triton-X100 and subsequently incubated in blocking buffer (PBS+0.1\% Tween-20+3\% BSA) for 2 hours. All subsequent steps are performed in blocking buffer. Samples are incubated with primary antibody for 1 hour and incubated with secondary antibodies and DAPI for $30 \mathrm{~min}$. After antibody incubations, samples were washed three times with PBS+0.1\% Tween-20 and once in milliQ before being mounted on microscopy slides using FluorSafe (Calbiochem). Cells were examined by confocal microscopy (Leica SPE-II) and Leica Application Suite Advanced Fluorescence software (LAS-AF).

Quantification of SGs. We analyzed the number of SGs and their surface area in $\sim 100$ cells per condition, the combined total of 10-20 images, using ImageJ software. For each image, background signal was removed by creating a blurred duplicate and subtracting it from the original image. Subsequently, remaining diffuse (cytoplasmic) SG marker signal was removed via weak blurring, adjustment of the contrast settings and applying a black-andwhite threshold. In the resulting image, the number and average surface area of the SGs (shown in black on a white background) were quantified. The used macro is available upon request. Subsequent statistical analyses were performed using GraphPad Prism software. Error bars represent standard deviations, p-values were calculated using one-way ANOVA analysis with Bonferroni post-hoc test (infections with recombinant EMCVs) or a onetailed t-test (FMDV infections).

Flow cytometry analysis. HeLa R19 cells were seeded in 12 -wells plates (200,000 cells/well) and the next day infected with the indicated viruses (MOI 10). Six hpi cells were trypsinized and resuspended in FACS buffer (PBS + $1 \%$ BSA). Cells were fixed for 30 minutes in $2 \%$ paraformaldehyde in FACS buffer and subsequently fixed in ice-cold methanol for 10 minutes. All subsequent steps are performed in FACS buffer. Cells were stained with primary antibodies for 1 hour. Subsequently cells were washed three times and incubated in secondary antibodies, in the dark, for $30 \mathrm{~min}$. Cells were washed three times and kept in $1 \%$ paraformaldehyde until analysis on FACS Canto II (BD biosciences) using BD FACS Diva software. Data analysis was performed using FlowJo software.

Western Blot analysis. HeLa R19 cells were seeded in $10 \mathrm{~cm}$ dishes and the next day infected (MOI 10) with the indicated viruses. At the indicated time points cells were released using trypsin, washed once in PBS and lysed in $100 \mu$ l lysisbuffer (100 mM Tris pH 8.0, 1 mM EDTA, $50 \mathrm{mM} \mathrm{NaCl}, 1 \% \mathrm{NP} 40$, protease inhibitor mix (Roche). Post nuclear lysate was obtained by centrifugation at $15000 \mathrm{xg}$ at $4^{\circ} \mathrm{C}$ for $15 \mathrm{~min}$. The amount of total protein in the lysates was determined using BCA assay (ThermoFisher). $100 \mu \mathrm{g}$ protein from the cleared cell lysates were resolved using reducing sodiumdodecyl sulfate-polyacrylamide gel electrophoresis (SDS-PAGE) and transferred to $0.2 \mu \mathrm{m}$ nitrocellulose membranes by wet electrophoretic transfer. Membranes were incubated ih in blocking buffer (PBS $+0.1 \%$ Tween $20+2 \%$ BSA) and successively incubated overnight with primary antibodies diluted in blocking buffer and then for $30 \mathrm{~min}$ with respective secondary antibodies diluted in blocking buffer. Between and after the incubations, the membranes were washed three times with PBS+0.1\% Tween-20. Finally, membranes were washed once with PBS and scanned using an Odyssey Imager (Li-COR). For the analysis of protein of FMDV-infected cells, $40 \mathrm{ng}$ of protein was resolved by SDS-PAGE, transferred by western blot and secondary antibodies conjugated with horseradish peroxidase (Pierce) were used for detection of proteins. Following incubation with appropriate primary and secondary antibodies, protein bands were visualized using SuperSigna ${ }^{\circledR}$ West Dura Extended Duration Substrate (ThermoScientific, Rockford, IL, USA) according to the manufacturer's directions. 


\section{Acknowledgments}

We are grateful to Martijn van Hemert for the kind gift of the pCMV-Flag-G3BP1 and pCMV-Flag-G3BP2 expression plasmids. The work was supported by a Vici grant (NWO918.12.628) from the Netherlands Organization for Scientific Research. Martijn A. Langereis is supported by a Veni grant (NWO-863.13.008) and Linda J. Visser is supported by the NWO-graduate program Infection and Immunity (NWO-022.004.018), both from the Netherlands Organization for Scientific Research. Gisselle N. Medina and Teresa de los Santos were supported by CRIS project $\mathrm{N}^{\circ}$ 8064-320000-061-00D from the U.S. Department of Agriculture, Agricultural Research Service.

\section{References}

1. Wu J, Chen ZJ. 2014. Innate Immune Sensing and Signaling of Cytosolic Nucleic Acids. Annu Rev Immunol 32:461-488.

2. McCormick C, Khaperskyy DA. 2017. Translation inhibition and stress granules in the antiviral immune response. Nat Publ Gr 17:647-660.

3. Pakos-Zebrucka K, Koryga I, Mnich K, Ljujic M, Samali A, Gorman AM. 2016. The integrated stress response. EMBO Rep 17:1374-1395.

4. Kedersha N, Ivanov P, Anderson P. 2013. Stress granules and cell signaling: More than just a passing phase? Trends Biochem Sci 38:494-506.

5. Reineke LC, Lloyd RE. 2015. The stress granule protein $\mathrm{G}_{3} \mathrm{BP}$ 1 recruits protein kinase $\mathrm{R}$ to promote multiple innate immune antiviral responses. J Virol 89:2575-89.

6. Reineke LC, Kedersha N, Langereis MA, van Kuppeveld FJMM, Lloyd RE. 2015. Stress granules regulate double-stranded RNA-dependent protein kinase activation through a complex containing $\mathrm{G}_{3} \mathrm{BP} 1$ and Caprini. MBio 6:02486-14.

7. Reineke LC, Dougherty JD, Pierre P, Lloyd RE. 2012. Large G3BP-induced granules trigger eIF2 phosphorylation. Mol Biol Cell 23:3499-3510.

8. Onomoto K, Jogi M, Yoo JS, Narita R, Morimoto S, Takemura A, Sambhara S, Kawaguchi A, Osari S, Nagata K, Matsumiya T, Namiki H, Yoneyama M, Fujita T. 2012. Critical role of an antiviral stress granule containing RIG-I and PKR in viral detection and innate immunity. PLoS One 7:e43031.

9. Langereis MA, Feng $Q$ van Kuppeveld FJ. 2013. MDA5 localizes to stress granules, but this localization is not required for the induction of type I interferon. J Virol 87:6314-25.

10. Kim WJ, Back SH, Kim V, Ryu I, Jang SK. 2005. Sequestration of TRAF2 into stress granules interrupts tumor necrosis factor signaling under stress conditions. Mol Cell Biol 25:2450-62.

11. Humoud MN, Doyle N, Royall E, Willcocks MM, Sorgeloos F, van Kuppeveld F, Roberts LO, Goodfellow IG, Langereis MA, Locker N. 2016. Feline Calicivirus Infection Disrupts Assembly of Cytoplasmic Stress Granules and Induces $\mathrm{G}_{3} \mathrm{BP} 1$ Cleavage. J Virol 90:6489-501.

12. Garaigorta U, Heim MH, Boyd B, Wieland S, Chisari F V. 2012. Hepatitis C virus (HCV) induces formation of stress granules whose proteins regulate HCV RNA replication and virus assembly and egress. J Virol 86:11043-56.

13. Xia J, Chen X, Xu F, Wang Y, Shi Y, Li Y, He J, Zhang P. 2015. Dengue virus infection induces formation of G3BP1 granules in human lung epithelial cells. Arch Virol 160:2991-2999.

14. Bidet K, Dadlani D, Garcia-Blanco MA. 2014. G3BP1, G3BP2 and CAPRIN1 Are Required for Translation of Interferon Stimulated mRNAs and Are Targeted by a Dengue Virus Non-coding RNA. PLoS Pathog 10:e1004242.

15. Panas MD, Varjak M, Lulla A, Eng KE, Merits A, Karlsson Hedestam GB, McInerney GM. 2012. Sequestration of $\mathrm{G}_{3} \mathrm{BP}$ coupled with efficient translation inhibits stress granules in Semliki Forest virus infection. Mol Biol Cell 23:4701-12.

16. Scholte FEM, Tas A, Albulescu IC, Žusinaite E, Merits A, Snijder EJ, van Hemert MJ. 2015. Stress granule components $\mathrm{G}_{3} \mathrm{BP1}$ and $\mathrm{G}_{3} \mathrm{BP} 2$ play a proviral role early in Chikungunya virus replication. J Virol 89:4457-69.

17. Fros JJ, Domeradzka NE, Baggen J, Geertsema C, Flipse J, Vlak JM, Pijlman GP. 2012. Chikungunya virus nsP3 blocks stress granule assembly by recruitment of $\mathrm{G} 3 \mathrm{BP}$ into cytoplasmic foci. J Virol 86:10873-9.

18. Feng $\mathrm{Q}$, Langereis MA, Lork M, Nguyen M, Hato S V, Lanke K, Emdad L, Bhoopathi P, Fisher PB, Lloyd RE, van Kuppeveld FJM. 2014. Enterovirus 2Apro targets MDA5 and MAVS in infected cells. J Virol 88:3369-78.

19. Rui Y, Su J, Wang H, Chang J, Wang S, Zheng W, Cai Y, Wei W, Gordy JT, Markham R, Kong W, Zhang W, Yu X-F. 2017. Disruption of MDA5 mediated innate immune responses by the 3C proteins of Coxsackievirus A16, Coxsackievirus A6, and Enterovirus D68. J Virol 91:e00546-17. 
20. Barral PM, Sarkar D, Fisher PB, Racaniello VR. 2009. RIG-I is cleaved during picornavirus infection. Virology 391:171-176.

21. Wang B, Xi X, Lei X, Zhang X, Cui S, Wang J, Jin Q Zhao Z. 2013. Enterovirus 71 Protease 2Apro Targets MAVS to Inhibit Anti-Viral Type I Interferon Responses. PLoS Pathog 9:e1003231.

22. Mukherjee A, Morosky SA, Delorme-Axford E, Dybdahl-Sissoko N, Oberste MS, Wang T, Coyne CB. 2011. The coxsackievirus B 3 Cpro protease cleaves MAVS and TRIF to attenuate host type I interferon and apoptotic signaling. PLoS Pathog 7:e1001311.

23. Wang C, Sun M, Yuan X, Ji L, Jin Y, Cardona CJ, Xing Z. 2017. Enterovirus 71 suppresses interferon responses by blocking Janus kinase (JAK)/signal transducer and activator of transcription (STAT) signaling through inducing karyopherin- $\alpha 1$ degradation. J Biol Chem 292:10262-10274.

24. White JP, Cardenas AM, Marissen WE, Lloyd RE. 2007. Inhibition of Cytoplasmic mRNA Stress Granule Formation by a Viral Proteinase. Cell Host Microbe 2:295-305.

25. Dougherty JD, Tsai WC, Lloyd RE. 2015. Multiple poliovirus proteins repress cytoplasmic RNA granules. Viruses 7:6127-6140.

26. Borghese F, Michiels T. 2011. The Leader Protein of Cardioviruses Inhibits Stress Granule Assembly. J Virol 85:9614-9622.

27. Feng $Q$ L Langereis MA, van Kuppeveld FJM. 2014. Induction and suppression of innate antiviral responses by picornaviruses. Cytokine Growth Factor Rev 25:577-585.

28. Ng CS, Jogi M, Yoo J-S, Onomoto K, Koike S, Iwasaki T, Yoneyama M, Kato H, Fujita T. 2013. Encephalomyocarditis virus disrupts stress granules, the critical platform for triggering antiviral innate immune responses. J Virol 87:9511-22.

29. Guarné A, Tormo J, Kirchweger R, Pfistermueller D, Fita I, Skern T. 1998. Structure of the foot-and-mouth disease virus leader protease: $\mathrm{A}$ papain-like fold adapted for self-processing and eIF4G recognition. EMBO J 17:7469-7479.

30. Brown CC, Piccone ME, Mason PW, McKenna TS, Grubman MJ. 1996. Pathogenesis of wild-type and leaderless foot-and-mouth disease virus in cattle. J Virol 70:5638-41.

31. de Los Santos T, de Avila Botton S, Weiblen R, Grubman MJ. 2006. The leader proteinase of foot-and-mouth disease virus inhibits the induction of beta interferon $\mathrm{mRNA}$ and blocks the host innate immune response. J Virol 80:1906-14.

32. de Los Santos T, Diaz-San Segundo F, Grubman MJ. 2007. Degradation of nuclear factor kappa B during foot-and-mouth disease virus infection. J Virol 81:12803-12815.

33. Wang D, Fang L, Luo R, Ye R, Fang Y, Xie L, Chen H, Xiao S. 2010. Foot-and-mouth disease virus leader proteinase inhibits dsRNA-induced type I interferon transcription by decreasing interferon regulatory factor 3/7 in protein levels. Biochem Biophys Res Commun 399:72-78.

34. Wang D, Fang L, Li P, Sun L, Fan J, Zhang Q, Luo R, Liu X, Li K, Chen H, Chen Z, Xiao S. 2011. The Leader Proteinase of Foot-and-Mouth Disease Virus Negatively Regulates the Type I Interferon Pathway by Acting as a Viral Deubiquitinase. J Virol 85:3758-3766.

35. Swatek KN, Aumayr M, Pruneda JN, Visser LJ, Berryman S, Kueck AF, Geurink PP, Ovaa H, van Kuppeveld FJM, Tuthill TJ, Skern T, Komander D. 2018. Irreversible inactivation of ISG15 by a viral leader protease enables alternative infection detection strategies. Proc Natl Acad Sci U S A 115:2371-2376.

36. Bailey-Elkin BA, Knaap RCM, Kikkert M, Mark BL. 2017. Structure and Function of Viral Deubiquitinating Enzymes. J Mol Biol 429:3441-3470.

37. Wang D, Fang L, Li K, Zhong H, Fan J, Ouyang C, Zhang H, Duan E, Luo R, Zhang Z, Liu X, Chen H, Xiao S. 2012. Foot-and-mouth disease virus $3 \mathrm{C}$ protease cleaves NEMO to impair innate immune signaling. J Virol 86:9311-9322.

38. Du Y, Bi J, Liu J, Liu X, Wu X, Jiang P, Yoo D, Zhang Y, Wu J, Wan R, Zhao X, Guo L, Sun W, Cong X, Chen L, Wang J. 2014. 3Cpro of foot-and-mouth disease virus antagonizes the interferon signaling pathway by blocking STATi/STAT2 nuclear translocation. J Virol 88:4908-20.
39. Monaghan P, Gold S, Simpson J, Zhang Z, Weinreb PH, Violette SM, Alexandersen S, Jackson T. 2005. The av $\beta 6$ integrin receptor for Foot-and-mouth disease virus is expressed constitutively on the epithelial cells targeted in cattle. J Gen Virol 86:2769-2780.

40. LaRocco M, Krug PW, Kramer E, Ahmed Z, Pacheco JM, Duque H, Baxt B, Rodriguez LL. 2013. A continuous bovine kidney cell line constitutively expressing bovine $\alpha V \beta 6$ integrin has increased susceptibility to footand-mouth disease virus. J Clin Microbiol 51:1714-1720.

41. Hato S V, Ricour C, Schulte BM, Lanke KHW, de Bruijn M, Zoll J, Melchers WJG, Michiels T, van Kuppeveld FJM. 2007. The mengovirus leader protein blocks interferon-alpha/beta gene transcription and inhibits activation of interferon regulatory factor 3. Cell Microbiol 9:2921-2930.

42. Feng Q Hato SVV., Langereis MA, Zoll J, Virgen-Slane R, Peisley A, Hur S, Semler BLL, van Rij RP, van Kuppeveld FJM. 2012. MDA5 Detects the Double-Stranded RNA Replicative Form in Picornavirus-Infected Cells. Cell Rep 2:1187-1196.

43. Rabouw HH, Langereis MA, Knaap RCM, Dalebout TJ, Canton J, Sola I, Enjuanes L, Bredenbeek PJ, Kikkert M, de Groot RJ, van Kuppeveld FJM. 2016. Middle East Respiratory Coronavirus Accessory Protein 4a Inhibits PKR-Mediated Antiviral Stress Responses. PLoS Pathog 12:e1005982.

44. Kaminski A, Belsham GJ, Jackson RJ. 1994. Translation of encephalomyocarditis virus RNA: parameters influencing the selection of the internal initiation site. EMBO J 13:1673-81.

45. Hato SV., Sorgeloos F, Ricour C, Zoll J, Melchers WJG, Michiels T, van Kuppeveld FJM. 2010. Differential IFN$\alpha / \beta$ production suppressing capacities of the leader proteins of mengovirus and foot-and-mouth disease virus. Cell Microbiol 12:310-317.

46. Piccone ME, Rieder E, Mason PW, Grubman MJ. 1995. The foot-and-mouth disease virus leader proteinase gene is not required for viral replication. J Virol 69:5376-5382.

47. Fung G, Ng CS, Zhang J, Shi J, Wong J, Piesik P, Han L, Chu F, Jagdeo J, Jan E, Fujita T, Luo H. 2013. Production of a dominant-negative fragment due to $\mathrm{G} 3 \mathrm{BP}$ cleavage contributes to the disruption of mitochondriaassociated protective stress granules during CVB3 infection. PLoS One 8:e79546.

48. Yang X, Hu Z, Fan S, Zhang Q Zhong Y, Guo D, Qin Y, Chen M. 2018. Picornavirus $2 A$ protease regulates stress granule formation to facilitate viral translation. PLOS Pathog 14:e1006901.

49. Kedersha N, Panas MD, Achorn CA, Lyons S, Tisdale S, Hickman T, Thomas M, Lieberman J, McInerney GM, Ivanov P, Anderson P. 2016. G3BP-Caprini-USP10 complexes mediate stress granule condensation and associate with 40S subunits. J Cell Biol 212:845-60.

50. Agol VI, Gmyl AP. 2010. Viral security proteins: counteracting host defences. Nat Rev Microbiol 8:867-78.

51. Rai DK, Lawrence P, Kloc A, Schafer E, Rieder E. 2015. Analysis of the interaction between host factor Sam68 and viral elements during foot-and-mouth disease virus infections. Virol J 12:224.

52. Lawrence P, Schafer EA, Rieder E. 2012. The nuclear protein Sam68 is cleaved by the FMDV 3 C protease redistributing Sam68 to the cytoplasm during FMDV infection of host cells. Virology 425:40-52.

53. Zhu Y, Wang B, Huang H, Zhao Z. 2016. Enterovirus 71 induces anti-viral stress granule-like structures in RD cells. Biochem Biophys Res Commun 476:212-217.

54. Zhang H, Chen N, Li P, Pan Z, Ding Y, Zou D, Li L, Xiao L, Shen B, Liu S, Cao H, Cui Y. 2016. The nuclear protein Sam 68 is recruited to the cytoplasmic stress granules during enterovirus 71 infection. Microb Pathog 96:58-66.

55. Black TL, Safer B, Hovanessian A, Katze MG. 1989. The cellular 68,000-Mr protein kinase is highly autophosphorylated and activated yet significantly degraded during poliovirus infection: implications for translational regulation. J Virol 63:2244-51.

56. Chang Y-H, Lau KS, Kuo R-L, Horng J-T. 2017. dsRNA Binding Domain of PKR Is Proteolytically Released by Enterovirus A71 to Facilitate Viral Replication. Front Cell Infect Microbiol 7:284.

57. Li C, Zhu Z, Du X, Cao W, Yang F, Zhang X, Feng H, Li D, Zhang K, Liu X, Zheng H. 2017. Foot-and-mouth disease virus induces lysosomal degradation of host protein kinase PKR by $3 \mathrm{C}$ proteinase to facilitate virus replication. Virology 509:222-231. 
Chapter 2 - Aphthovirus L ${ }^{\text {pro }}$ Inhibits Stress Granule Formation

58. Chinsangaram J, Koster M, Grubman MJ. 2001. Inhibition of L-deleted foot-and-mouth disease virus replication by alpha/beta interferon involves double-stranded RNA-dependent protein kinase. J Virol 75:5498-503.
replication by alpha/beta interferon involves double-stranded RNA-dependent protein kinase. J Virol
.

Galan A, Lozano G, Piñeiro D, Martinez-Salas E. 2017. G3BP1 interacts directly with the FMDV IRES and negatively regulates translation. FEBS J 284:3202-3217.

60. Rieder E, Bunch T, Brown F, Peter Mason AndW. 1993. Genetically Engineered Foot-and-Mouth Disease Viruses with Poly(C) Tracts of Two Nucleotides Are Virulent in Mice. J Virol 67:5139-5145.

61. Van Der Schaar HM, Leyssen P, Thibaut HJ, De Palma A, Van Der Linden L, Lanke KHW, Lacroix C, Verbeken E, Conrath K, MacLeod AM, Mitchell DR, Palmer NJ, Van De Poel H, Andrews M, Neyts J, Van Kuppeveld
FJM. 2013. A Novel, Broad-Spectrum Inhibitor of Enterovirus Replication That Targets Host Cell Factor Phosphatidylinositol 4-Kinase III-beta. Antimicrob Agents Chemother 57:4971-4981.

62. Van Der Linden L, Ulferts R, Nabuurs SB, Kusov Y, Liu H, George S, Lacroix C, Goris N, Lefebvre D, Lanke KHW, De Clercq K, Hilgenfeld R, Neyts J, Van Kuppeveld FJM. 2014. Application of a cell-based protease assay for testing inhibitors of picornavirus 3 C proteases. Antiviral Res 103:17-24. 


\section{Chapter 3}

Irreversible Inactivation of ISG15 by a Viral Leader Protease Enables Alternative Infection Detection Strategies

Kirby N. Swatek ${ }^{1}$, Martina Aumayr 2,", Jonathan N. Pruneda ${ }^{1, "}$, Linda J. Visser ${ }^{3, "}$, Stephen Berryman ${ }^{4}$, Anja F. Kueck ${ }^{1}$, Paul P. Geurink ${ }^{5}$, Huib Ovaa ${ }^{5}$, Frank J. M. van Kuppeveld ${ }^{3}$, Tobias J. Tuthill ${ }^{4}$, Tim Skern ${ }^{2}$, David Komander ${ }^{1, *}$

${ }^{1}$ Protein and Nucleic Acid Chemistry Division, Medical Research Council Laboratory of Molecular Biology, CB2 OQH Cambridge, United Kingdom

${ }^{2}$ Departmentof Medical Biochemistry, Max F. Perutz Laboratories, Vienna Biocenter, Medical University of Vienna, A-1030 Vienna, Austria

${ }^{3}$ Virology Division, Department of Infectious Diseases and Immunology, Faculty of Veterinary Medicine, Utrecht University, The Netherlands

${ }^{4}$ The Pirbright Institute, GU24 ONFPirbright, Surrey, United Kingdom

${ }^{5}$ Department of Chemical Immunology, Leiden University Medical Centre, 2333 ZC Leiden, The Netherlands

"These authors contributed equally.

* Corresponding author. 


\section{Abstract}

In response to viral infection, cells mount a potent inflammatory response that relies on ISG15 and ubiquitin posttranslational modifications. Many viruses use deubiquitinases and deISGylases that reverse these modifications and antagonize host signaling processes. We here reveal that the leader protease, $\mathrm{Lb}^{\text {pro }}$, from foot-and-mouth disease virus (FMDV) targets ISG15 and to a lesser extent, ubiquitin in an unprecedented manner. Unlike canonical deISGylases that hydrolyze the isopeptide linkage after the C-terminal GlyGly motif, Lb ${ }^{\text {pro }}$ cleaves the peptide bond preceding the GlyGly motif. Consequently, the GlyGly dipeptide remains attached to the substrate Lys, and cleaved ISG15 is rendered incompetent for reconjugation. A crystal structure of Lb ${ }^{\text {pro }}$ bound to an engineered ISG15 suicide probe revealed the molecular basis for ISG15 proteolysis. Importantly, anti-GlyGly antibodies, developed for ubiquitin proteomics, are able to detect $\mathrm{Lb}^{\text {pro }}$ cleavage products during viral infection. This opens avenues for infection detection of FMDV based on an immutable, host-derived epitope.

\section{Significance}

An understanding of the mechanisms by which viruses evade host immunity is essential to the development of antiviral drugs and viral detection strategies. Ubiquitin and ubiquitinlike modifications are crucial in cellular innate immune and infection responses and are often suppressed by viral proteins. We here identify a previously unknown mechanism of viral evasion. A viral protease, $\mathrm{Lb}^{\text {pro }}$, removes ubiquitin and the ubiquitin-like protein ISGI5 incompletely from proteins. While this strategy efficiently and irreversibly shuts down these modification systems, it enables repurposing of tools and technologies developed for ubiquitin research in virus detection. Specifically, we show that foot-and-mouth disease virus infection can be detected using an anti-GlyGly antibody developed for ubiquitin mass spectrometry research.

\section{Introduction}

Picornaviruses are nonenveloped, positive-strand RNA viruses that comprise small genomes but represent significant threats to human and animal health (1). For example, foot-and-mouth disease virus (FMDV) is a global, highly infectious animal pathogen that causes annual economic losses in livestock farming estimated between $\$ 6.5$ and $\$ 21$ billion US dollars (2). Infected cattle develop fever and vesicular lesions in their mouths and hooves; consequently, FMDV outbreaks lead to culling of entire herds in FMDV-free countries to prevent the spread of the virus (3).

Among the 14 mature proteins encoded by FMDV are two peptidase activities required for self-processing and viral proliferation $(4,5)$. The leader protease $\mathrm{Lb}^{\text {pro }}$ is a papainlike cysteine protease that has several roles $(4,6)$. First, $\mathrm{Lb}^{\text {pro }}$ cleaves its own $\mathrm{C}$ terminus, releasing itself from the nascent polyprotein. Second, $\mathrm{Lb}^{\text {pro }}$ also cleaves the two isoforms of eukaryotic initiation factor $4 \mathrm{G}$ (eIF4G), the inactivation of which diverts ribosomes to preferentially translate viral RNA via their internal ribosome entry site $(4,6)$. Third, $\mathrm{Lb}^{\mathrm{pro}}$ was reported to subvert innate immune signaling pathways by acting as a deubiquitinase $(\mathrm{DUB})(7)$.

Ubiquitination is a key signaling mechanism used by cells to detect and respond to viral infection (8). Various ubiquitin signals play important roles during the activation of transcriptional programs, such as the NF- $\mathrm{KB}$ and IFN response $(8,9)$. With the initiation of IFN signaling, the products of roughly 300 IFN stimulated genes (ISGs) mount an antiviral response (10). Among the ISGs are the ubiquitin-like modifier ISG15 and its assembly machinery consisting of UBE1L, UBE2L6/UbcH8, and the HECT E3 ligase HERC5 (11). ISG15 comprises two ubiquitin fold domains in tandem [an N-terminal ubiquitin-like domain (NTD) and a C-terminal ubiquitin-like domain (CTD)], and like ubiquitin, it is attached to Lys residues on target proteins via its C-terminal Gly-Gly (Gly156-Gly157) motif. Cotranslational attachment of ISG15 to viral capsid proteins inhibits virus assembly (12), and additional intra- and extracellular roles of ISG15 are also emerging (13, 14). Together, ubiquitin and ubiquitin-like modifications regulate most antiviral signaling cascades.

Viruses are well-known to counter these defenses and manipulate the regulatory machineries or the modifications themselves $(8,9,15)$. For instance, Crimean Congo hemorrhagic fever virus (CCHFV), severe acute respiratory syndrome coronavirus, and Middle East respiratory syndrome coronavirus all encode proteases with dual deISGylase and DUB activity (16). 
We here show that FMDV Lb ${ }^{\text {pro }}$ targets ISG15 with strong preference over ubiquitin and NEDD8 and characterize this specificity biochemically and structurally. We uncover a previously undescribed mechanism by which viruses interfere with the ubiquitin and ubiquitin-like systems. Unlike canonical deISGylases, $\mathrm{Lb}^{\text {pro }}$ does not target the isopeptide bond formed during attachment but selectively cleaves a peptide bond in the $\mathrm{C}$ terminus of ISG15, which results in incomplete removal of the modifier. A crystal structure of $\mathrm{Lb}^{\text {pro }}$ covalently bound to a specifically designed ISG15 suicide probe reveals the molecular basis of $\mathrm{Lb}^{\mathrm{pro}}$ specificity and substrate promiscuity. Importantly, cleavage by $\mathrm{Lb}^{\text {pro }}$ exposes a GlyGly epitope on substrates of the modifier, and we show that this epitope can be detected in cells during FMDV infection with relative ease. Such mechanism- and activity-based detection strategy opens avenues for distinguishing infected from vaccinated animals and may help limit the economic impact of FMDV.

\section{Results}

$\mathbf{L b}^{\text {pro }}$ Substrate Specificity and Cleavage. We used ubiquitin and ubiquitin-like model substrates (17) to characterize Lb $b^{\text {pro }}$ DUB activity in vitro (Fig. S1A) and surprisingly found that while the enzyme lacked robust DUB activity, it targeted ISG15 with high activity and specificity (Fig. 1A). Ubiquitin cleavage required 1,000-fold higher enzyme concentration (Fig. S1B). Kinetic analysis revealed deISGylase activity comparable with previously studied viral $(18,19)$ and human enzymes $(20)$ (Fig. IB and Fig. SiC). However, a number of standard measurements for deISGylation activity were negative. ISG15-derived suicide probes that covalently modify ISG15-reactive Cys proteases $(21,22)$ were unable to modify Lb ${ }^{\text {pro }}$ (Fig. IC and Fig. S1D). Hydrolysis of ISG15 7-amido-4-methylcoumarin (AMC) by deISGylases, such as the viral ovarian tumor (vOTU) domain of CCHFV, leads to fluorescence, yet $\mathrm{Lb}^{\text {pro }}$ did not generate a fluorescent signal in this assay (Fig. S1 E and F). In contrast, slightly extended precursor forms of full-length proISG15 (amino acids 1-165; featuring an additional eight residues at the C terminus) or proISG15CTD (amino acids 79-165) were rapidly hydrolyzed by $\mathrm{Lb}^{\text {pro }}$ (Fig. 1D). Strikingly, mass spectrometry (MS) analysis of $\mathrm{Lb}^{\text {pro-treated mature }}$ ISG15CTD (amino acids 79-157) revealed a truncated ISG15 molecule; Lb ${ }^{\text {pro }}$ had cleaved ISG15 between Arg155 and the C-terminal Gly156-Gly157 sequence (Fig. I E and F).

This surprising result was corroborated in additional experiments. We compared the cleavage rates of pro-, mature-, and substrate-bound forms of ISG15 by intact MS analysis, revealing similar activity of Lb $\mathrm{b}^{\text {pro }}$ against pro-ISG15 and mature ISG15 and slightly higher activity toward ISG15 linked to 5-tetramethylrhodamine (TAMRA)-labeled Lys-Gly dipeptide via an isopeptide bond (Fig. $\mathrm{S} 1 \mathrm{G}$ ). This showed that $\mathrm{Lb}^{\text {pro }}$ can process all forms of ISG15 present in cells.
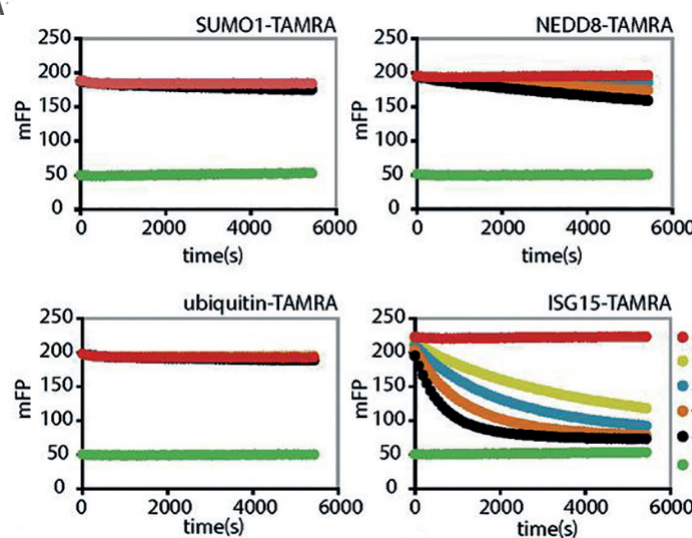

time(s)

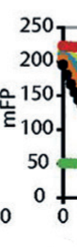

ISG15-TAM

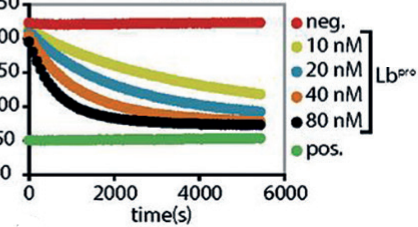

$20.00 \mathrm{nM}$ $.80 \mathrm{~nm}$

4000
time(s)

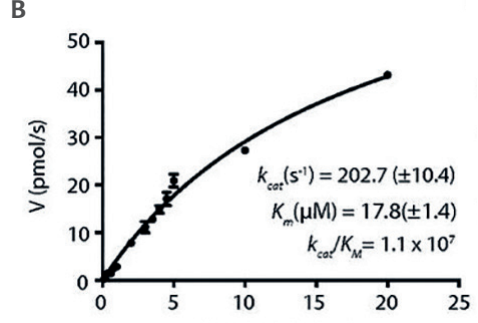

ISG15-TAMRA ( $\mu$ M)

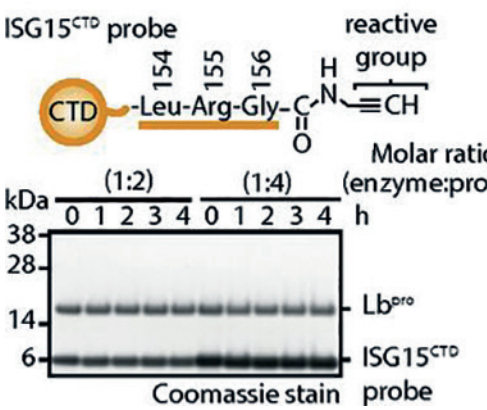

D
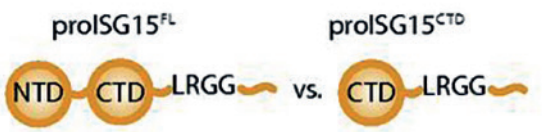

年

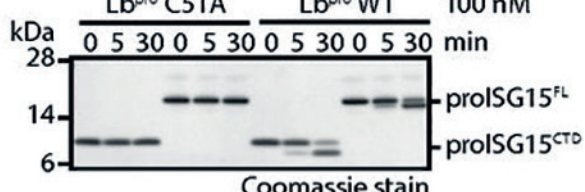

Coomassie stain

$E$
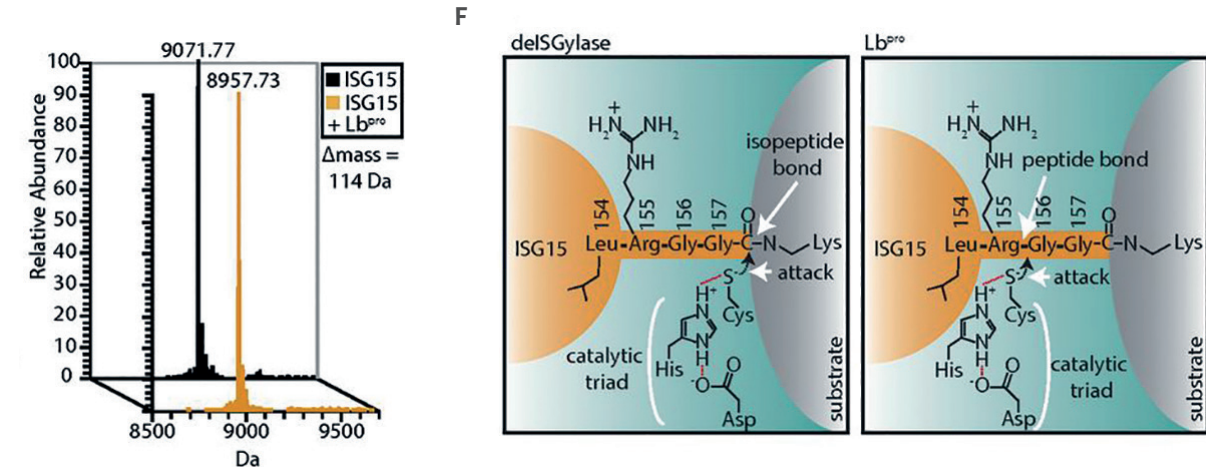

Figure 1: $L b^{\text {pro }}$ substrate specificity and cleavage. (A) Specificity analysis of $L b^{\text {pro }}$ against ubiquitin and ubiquitin-like (SUMO1, NEDD8, ISG15) TAMRA substrates (Fig. S1). (B) Michaelis-Menten kinetics of Lb pro as measured by ISG15TAMRA cleavage. Error bars represent SD from the mean. (C) Suicide ISG15 probe assays were performed with L $\mathrm{b}^{\text {pro }}$ and WT ISG15CTD probe. (D) Comparison of full-length prolSG15 (amino acids 1-165) and proISG15CTD (amino acids 79-165) cleavage by Lb ${ }^{\text {pro }}$. (E) Electrospray ionization MS of untreated and Lb pro-treated mature ISG15CTD (amino acids 79-157). The difference in mass corresponds to loss of the Gly-Cly peptide (114 Da). (F) Schematic of ISG15 cleavage by a canonical deISCylase (Left) and Lb bro (Right). delSCylases cleave isopeptide bonds, while $L b^{\text {pro }}$ cleaves the peptide bond C-terminal to Arg155, releasing a ClyCly dipeptide. All assays were performed in triplicate. NTD, N-terminal ubiquitin-like domain. 
The cleavage site of $\mathrm{Lb}^{\text {pro }}$ contrasts all known deISGylases, which cleave ISG15 from substrates, such that both proteins are recycled to the same state as they were before modification $(18,19)$. In hindsight, these findings explain the inability of $\mathrm{Lb}^{\text {pro }}$ to react with the ISG15 suicide probe (in which the reactive warhead would extend beyond the active site) or generate a signal in the ISG15-AMC assay. AMC only fluoresces in the unmodified form, whereas $\mathrm{Lb}^{\text {pro }}$ cleavage produces a nonfluorescent GlyGly-modified AMC reagent (Fig. S2A).

At higher enzyme concentrations, ubiquitin and NEDD8 were also cleaved at an identical site between Arg74 and the C-terminal GlyGly motif, whereas SUMO1 was not cleaved (Fig. S2 B-D). Hence, Lb ${ }^{\text {pro }}$ removes ISG15 (as well as ubiquitin and NEDD8) in a manner that leaves a GlyGly remnant attached to a substrate Lys residue and renders the ubiquitin/ ubiquitin-like protein incompetent toward additional rounds of conjugation.

Structure of Lb $\mathbf{L}^{\text {pro }}$ Bound to ISG15. To decipher the mechanism of this unprecedented activity, we designed an ISG15 suicide probe specific for Lb ${ }^{\text {pro }}$. As mentioned, the WT ISG15 suicide probe modified several viral proteases but was too long to modify Lb ${ }^{\text {pro }}$ (Fig. S2E). Removing the GlyGly motif from ISG15 ${ }^{\mathrm{CTD}}$ and replacing Arg155 with a Gly-like C-terminal propargyl warhead (Fig. 2A) generated an ISG $15^{{ }^{C T D}}-\Delta C$ probe that efficiently modified $\mathrm{Lb}^{\text {pro }}$ (Fig. $2 \mathrm{~B}$ and Fig. S2E). This enabled purification, crystallization, and structure determination of a covalent $\mathrm{Lb}^{\text {pro }} \sim \mathrm{ISG} 15^{\mathrm{CTD}}-\Delta \mathrm{C}$ complex at 1.5-Å resolution (Fig. $2 \mathrm{C}$ and D, Fig. S3, and Table S1). As anticipated, the ISG $15^{\mathrm{CTD}}-\Delta \mathrm{C}$ probe had covalently modified the catalytic Cys 51 of $\mathrm{Lb}^{\mathrm{pro}}$ (Fig. S3). Individually, the $\mathrm{Lb}^{\text {pro }}$ protease and ISG15 domains were similar to previously determined structures $(18,23)$ (C $\alpha$ rmsds of 0.609 and $0.274 \AA$, respectively).

The structure revealed how proteolytic cleavage of ISG15 is achieved (Fig. 2E and Fig. S3). Key interactions are formed via the ISG15 C terminus and also, via a hydrophobic surface centered on ISG15 Trp123 (Fig. S3). The positively charged residues of the ISG15 C terminus, Arg153 and Arg155 (the latter mutated to Gly in the ISG15 ${ }^{\mathrm{CTD}}-\Delta \mathrm{C}$ probe), are cradled by an acidic groove that consists of Asp49, Glu96, and Glu147 on Lb ${ }^{\text {pro }}$ (Fig. S3G). Furthermore, ISG15 Leu154 occupies a hydrophobic pocket commonly found in papain-like enzymes. Mutation of either Leu154 or Arg155 to Ala in proISG15 strongly reduced cleavage by Lb $b^{\text {pro }}$ (Fig. $\mathrm{S}_{3} \mathrm{H}$ ). However, these C-terminal mutations do not prevent ISG15 binding, since the ISG $15^{\text {CTD }}-\Delta$ C suicide probe that lacks Arg155 was able to modify Lb ${ }^{\text {pro }}$ (Fig. 2 B and C). The importance of the $\mathrm{C}$ terminus explains, at least in part, the observed weak cross-reactivity with ubiquitin and NEDD8, which feature identical or similar C-terminal sequences, respectively, and also, the inability of $\mathrm{Lb}^{\mathrm{pro}}$ to target other modifiers, such as SUMO1, which feature more divergent C-terminal sequences (24) (Fig. S3F). The observed interactions closely mimic those seen in the previously determined structure of $\mathrm{Lb}^{\text {pro }}$ carrying out selfprocessing of its own C terminus (23) (Fig. 2E and Fig. S3F).

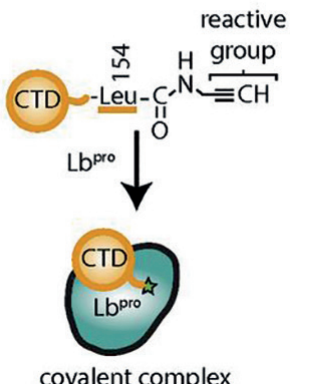

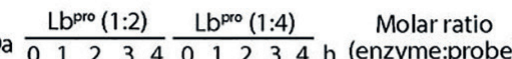

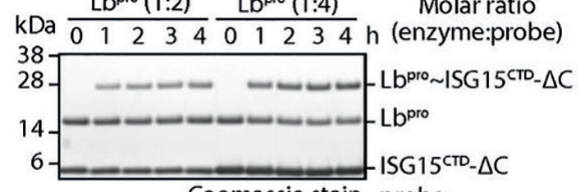

Coomassie stain probe

covalent complex

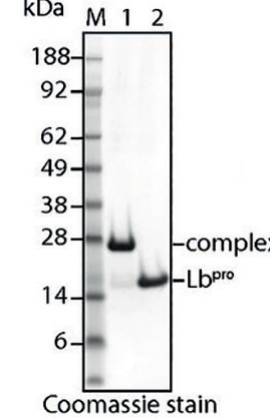

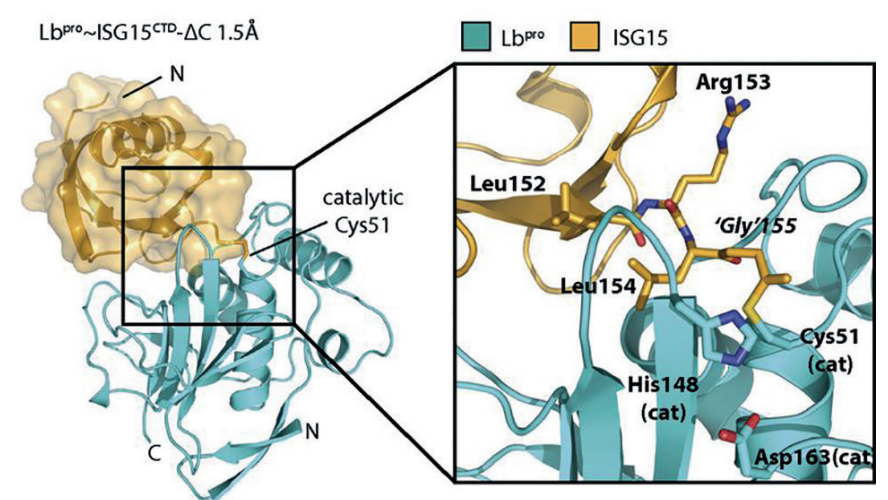

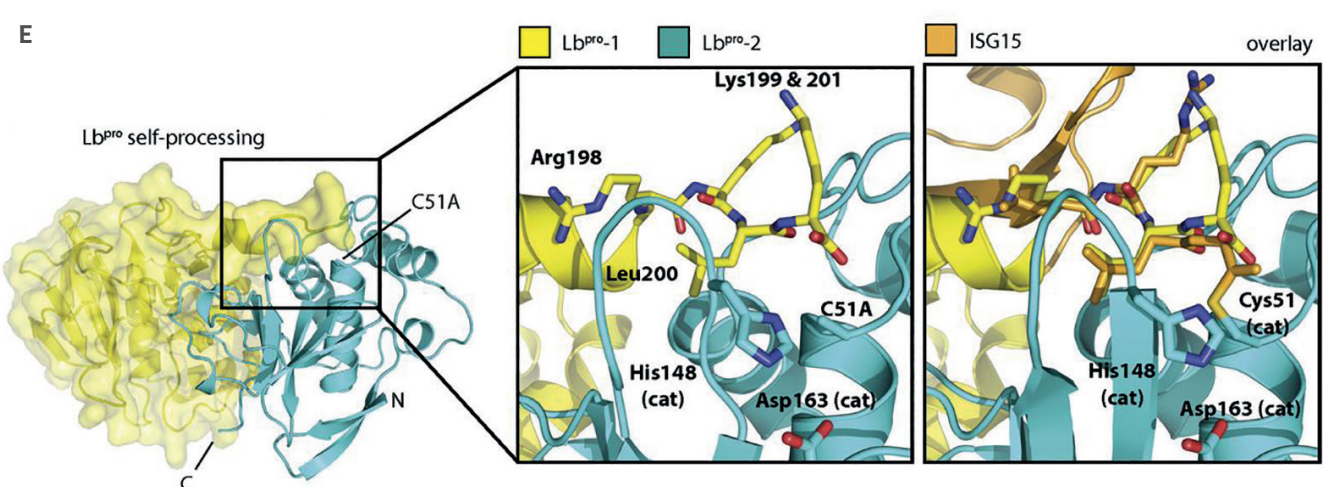

Figure 2: Structure of Lbro bound to ISG15. (A) Schematic of an engineered Lbprospecific ISG15 suicide probe (B) The ISG15 $15^{\mathrm{CT}}-\Delta \mathrm{C}$ suicide probe forms a covalent complex with $L b^{\text {pro }}$ (indicated by $\sim$ ). (C) SDS-PACE of the purified

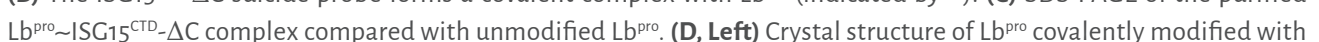

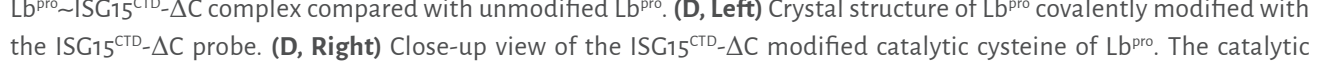
the ISG15 $15^{\mathrm{CT} D}-\Delta \mathrm{C}$ probe. (D, Right) Close-up view of the ISG15 $15^{\mathrm{CT} D}-\Delta \mathrm{C}$ modified catalytic cysteine of $\mathrm{Lb}^{\text {pro }}$. The catalytic
triad (Cys51, His148, Asp163) of Lb $\mathrm{p}^{\text {pro }}$ and C-terminal residues of ISG15 (amino acids 152-154; Arg155 was replaced by the Cly-like warhead) are highlighted. (E, Left) Structure of Lbpro (Protein Data Bank ID code 1qol) during self-processing in trans. The $L b^{\text {pro }}$ substrate positioned for trans self-processing (yellow; $\left.L b^{\text {pro }}-1\right)$ and the catalytic $L b^{\text {pro }}\left(c y a n ; L b^{\text {pro }}-2\right)$ are shown. (E, Center) $L b^{\text {pro }} C$-terminal residues of the substrate (L $\left.L b^{\text {pro }}-1\right)$ are shown (Arg198, Lys199, Leu200, Lys201), and residues of the catalytic triad are shown as in $D$ for $L b^{\text {pro }}-2$. (E, Right) The $L b^{\text {pro }} \sim I S C 15^{c T D}-\Delta C$ and $L b^{\text {pro }}$ self-processing structures were overlaid. Cel-based assays in B were performed in triplicate. 
Analysis of ISG15 Specificity. Another interaction site between ISG15 and Lbpro centers on the conserved Trp123 of ISG15 (Fig. 3A and Fig. S4A), the main hydrophobic ISG15 surface used in other deISGylases (Fig. S4B) $(18,19,22,25)$. Lb pro $^{\text {pro }}$ utilizes a corresponding hydrophobic ISG15 binding surface formed by Leu92, Pro99 in the $\alpha_{3} / \alpha_{4}$ loop, and residues, including Leu1o2 on the $\alpha_{4}$ helix to shape the overall structure of the binding site (Fig. 3A

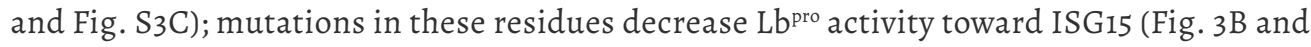
Fig. S4C). Importantly, this loop is not involved in interactions used during self-processing (Fig. 2E), and consequently and consistently, mutants in the ISG15 binding site are neither affected in their ability to self-process nor affected in their ability to cleave eIF4G (Fig. S5). Hence, substrate and ISG15 binding sites are spatially separated.

In ISG15, mutation of Trp123 to Ala leads to a significant reduction of cleavage by Lb ${ }^{\text {pro }}$ (Fig. 3C). Trpi23 is also a major difference between ISG15 and ubiquitin, where the structurally equivalent residue is Arg42 (Fig. 3D). Mutation of ubiquitin Arg42 to Trp in the context of an Met1-linked ubiquitin chain significantly reduced cleavage of this chain type by the Met1 linkage-specific DUB OTULIN (26) (Fig. S4D) but enhanced ubiquitin chain cleavage by Lb $b^{\text {pro }}$ (Fig. 3E and Fig. S4E). This reveals one component of how $\mathrm{Lb}^{\text {pro }}$ distinguishes modifiers.

Substrate Cleavage in Cells. Given the high level of $\mathrm{Lb}^{\mathrm{pro}}$ activity against ISG15 in vitro (Fig. 1 $A$ and $B$ ), we were curious whether this activity and noncanonical cleavage were detectable in a biological context. Transfection of FLAG-tagged ISG15 and the ISG15 assembly machinery (UBE1L, UBE2L6, and HERC5) led to robust ISGylation in HeLa cells. Treatment of these cell lysates with $\mathrm{Lb}^{\text {pro }}$ collapsed the ISGylated proteins, while free ISG15 remained seemingly unchanged (Fig. 4A and Fig. S6C). Serendipitously, we observed that ISG15 cross-reacted with a polyclonal antiubiquitin antibody (Fig. S6 A and B). Treatment with $\mathrm{Lb}^{\text {pro }}$ specifically removed this cross-reaction, indicating that a fraction of the polyclonal antibody detects an epitope spanning the identical C terminus of both modifiers (Fig. S6 $A$ and B). Antibody cross-reactivity provided us with a tool to monitor ISG15 cleavage by $\mathrm{Lb}^{\text {pro }}$ in cell extracts. Indeed, when transfection cell lysates were probed with the polyclonal anti-ubiquitin antibody, the ISG15 cross-reactive band was no longer detected after Lb $b^{\text {pro }}$ treatment, indicating that $\mathrm{Lb}^{\text {pro }}$ had hydrolyzed the ISG15 C terminus (Fig. 4A, red box). The antiubiquitin blot also showed that ubiquitin modifications in $\mathrm{Lb}^{\text {pro-treated samples }}$ were only partially processed, confirming ISG15 preference in complex samples (Fig. 4A). This was in stark contrast to the CCHFV vOTU domain, which cleaves ubiquitin and ISG15 similarly $(18,19)$ and collapsed both types of signals to the same extent (Fig. 4A).

To further visualize $L b^{\text {pro }}$ activity, we exploited a previously developed antibody that recognizes GlyGly-modified Lys residues $(27,28)$. This anti-GlyGly antibody has so far only been used to enrich ubiquitinated tryptic peptides in MS applications $(27,28)$. Rewardingly,
A
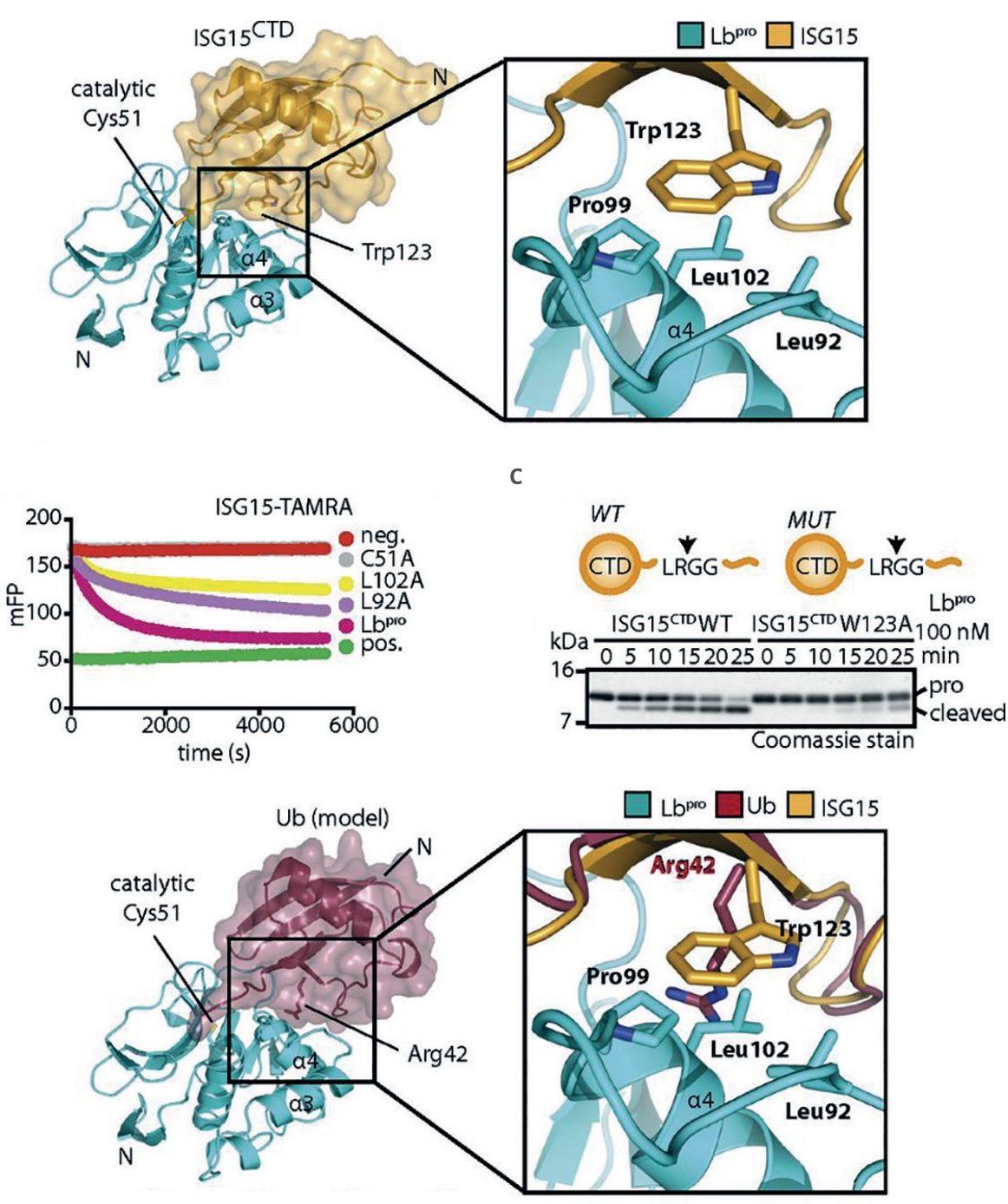

E

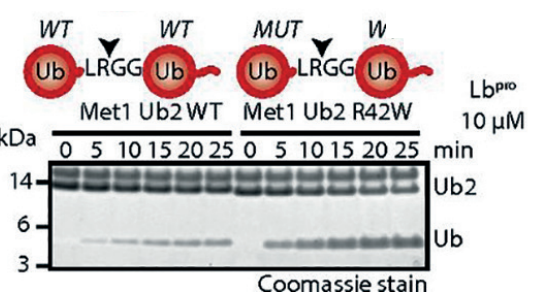

Figure 3: Structural analysis of ISG15 specificity. (A, Left) The L L pro $_{\text {pSSG15 }}{ }^{\mathrm{CT}}-\Delta \mathrm{C}$ complex is shown as in Fig. 2D. (A, Right) Close-up view of the hydrophobic contact between Lb ISG15-TAMRA cleavage assays with L $\mathrm{b}^{\text {pro }}$ point mutants in the hydrophobic patch. (C) L $\mathrm{b}^{\text {pro }}$ cleavage assays with WT and

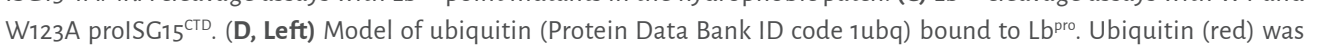
superimposed onto ISG15 $5^{\mathrm{CT} D}$. (D, Right) Close-up view of the analogous interface as in A. (E) Met1 diubiquitin cleavage assays with or without $\mathrm{R} 42 \mathrm{~W}$ mutation in the distal ubiquitin. Arrows in the schematics show the proteolytic site of cleavage (Fig. S4E). Assays in B, C, and E were performed in triplicate. 
probing these same reactions with the anti-GlyGly antibody produced a smear of strong

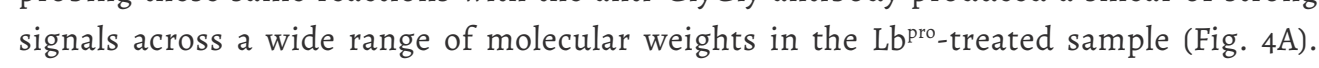
These signals correspond to the $\mathrm{Lb}^{\mathrm{pro}}$-produced GlyGly-modified proteome.

Revealing $\mathbf{L b}^{\text {pro }}$ Activity During Viral Infection. To assess the impact of Lb ${ }^{\text {pro }}$ activity during viral infection, we first exploited a chimera viral infection model (29). A mengovirus (a strain of encephalomyocarditis, a picornavirus that is closely related to FMDV) system was engineered, in which the Leader protein was inactivated by mutations and functionally replaced by WT $\mathrm{Lb}^{\text {pro }}$ or catalytically inactive $\mathrm{Lb}^{\text {pro }}$ Cys51Ala as a leader protease. We monitored Lbro activity in a time course with anti-ISG15, anti-ubiquitin, and anti-

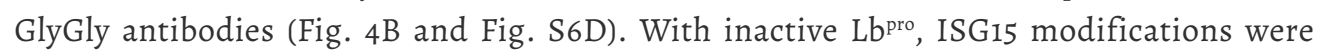
visibly increased $6-8 \mathrm{~h}$ postinfection, whereas no visible changes to total ubiquitin and no signal in the anti-GlyGly Western blot were apparent. In contrast, mengovirus with active $\mathrm{Lb}^{\text {pro }}$ resulted in a decrease in ISG15 signals, a slight decrease in ubiquitin signals, and importantly, the appearance of GlyGly-modified proteins at 6 and especially, $8 \mathrm{~h}$ postinfection (Fig. 4B and Fig. S6D). This indicates that, indeed, production of active $\mathrm{Lb}^{\text {pro }}$ by the virus leads to incomplete hydrolysis of ISG15 and ubiquitin from substrates, which can be visualized using available ubiquitin proteomics antibodies.

Importantly, Lb ${ }^{\text {pro }}$ activity can also be visualized during FMDV infection [e.g. using baby hamster kidney (BHK) cells transfected with FLAG-tagged ISG15 and the ISG15 conjugation system]. Before infection, transfection leads to robust FLAG signals, whereas anti-GlyGly signals are absent. FMDV infection induces collapsing of FLAG signals coinciding with the appearance of GlyGly-modified proteins. Within $4 \mathrm{~h}$ of FMDV infection, the anti-GlyGly antibody labels hundreds of proteins across a broad molecular weight range (Fig. $4 \mathrm{C}$ ). We are not aware of a similar host cell-derived viral-induced epitope that is detectable with such relative ease. Importantly, this epitope originates from host proteins and cannot acquire mutations due to viral evolution.

\section{Discussion}

$\mathrm{Lb}^{\text {pro }}$ is a prime example of a viral protein that contributes to successful viral replication through multifunctional roles. In addition to previously known crucial activities during replication $(4,6)$, we now show how it also hinders antiviral signaling through removal of ISG15 and to a lesser extent, ubiquitin from proteins (Fig. 5). Importantly, its mechanism of incomplete cleavage irreversibly damages the modifiers, which can no longer be attached to proteins. This mechanism is conceptually similar to the activity of RavZ, a Legionella effector that hydrolyzes the $\mathrm{C}$ terminus of Atg8 ubiquitin-like modifiers involved in

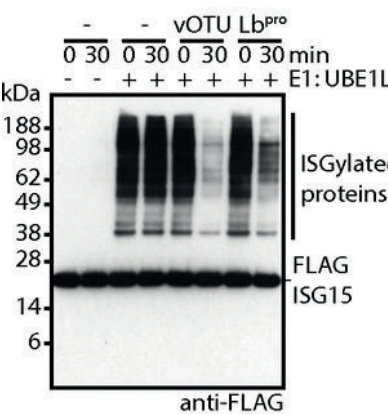

B

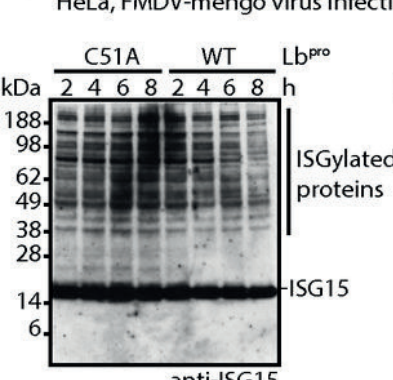

anti-ISG15 $\frac{-}{030} \frac{-}{030} \frac{\text { VOTU L }}{030} \frac{\text { pro }}{030} \min$

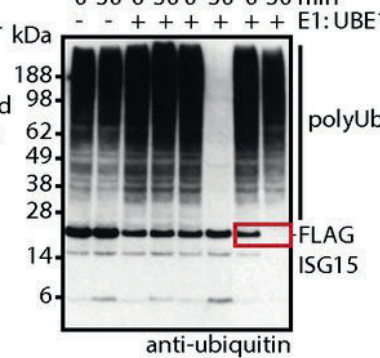

anti-ubiquitin

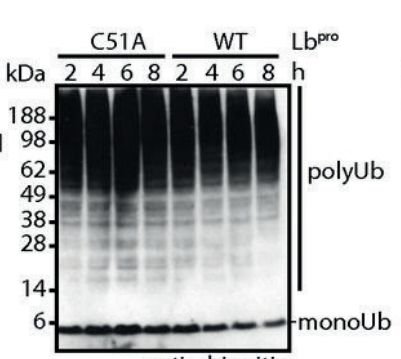

anti-ubiquitin $\frac{-}{030} \frac{-}{030} \frac{\text { VOTU }}{030} \frac{\mathrm{hb}^{\text {pro }}}{030}$

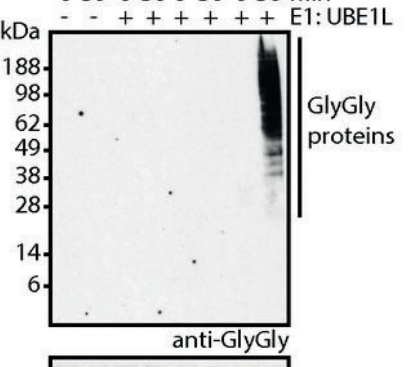

anti-GlyGly

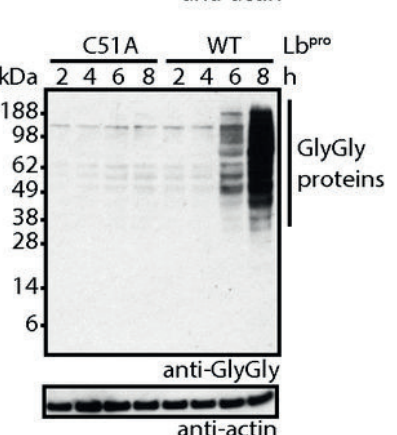

C BHK, FLAG-ISG15,

E1: UBE1L,
E3: HERC5

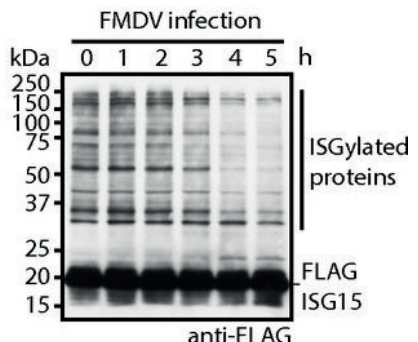

anti-FLAG

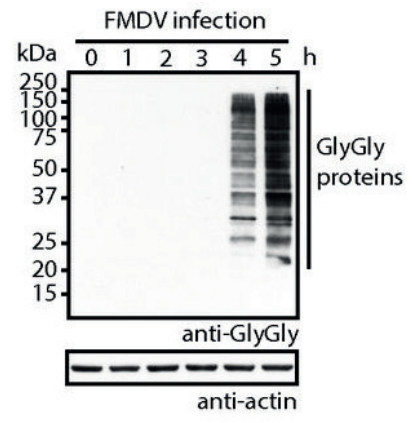

Figure 4:Validation of $L b^{\text {pro }}$ substrate cleavage in cells. (A) Lb pro cleavage assays of ISCylated proteins. HeLa cells were transfected with FLAG-ISG15 and the ISG15 conjugation machinery (UBE1L, UBE2L6, HERC5) to produce ISG15ylated proteins. (A, Left) Removal of ISG15 from substrates by Lb pro was monitored using an anti-FLAC antibody. (A, Center) A polyclonal antiubiquitin antibody monitors ubiquitin chain signals but can also be used to monitor cleavage of the

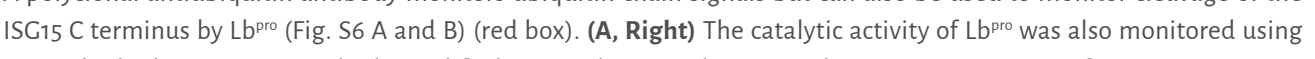
an antibody that recognizes ClyCly-modified Lys residues. Loading controls are in Fig. S6C. (B) Infection assays using an FMDV $L b^{\text {pro }}$-mengovirus chimera. The catalytic activity of $L b^{\text {pro }}$ was monitored as in $A$, with the difference being that ISG15 was detected using an anti-ISG15 antibody (Left). A catalytically inactive FMDV Lb bro-mengovirus (C51A) was used as a control. Fig. S6D shows loading controls. (C) FMDV infection time course using BHK fibroblast cells transfected with FLAC-ISC15 and the ISC15 conjugation machinery. Lbro catalytic activity was monitored with anti-FLAC (Top) and antiClyCly (Bottom) antibodies. All assays were performed in triplicate. 
autophagy (30). Moreover, on the substrate side, $\mathrm{Lb}^{\text {pro }}$ activity precludes remodification of Lys residues, and their small GlyGly modification(s) may not alter protein function significantly (Fig. 5). The slight cross-reactivity with ubiquitin is likely important, since ubiquitin modifications are much more abundant, and it is hence difficult to delineate the origin of the observed GlyGly signatures. Nonetheless, these multifaceted traits highlight the importance of $\mathrm{Lb}^{\text {pro }}$ as a potent virulence factor (31). It is possible that other viruses and pathogens may use this elegant anti-inflammatory strategy. While the leader proteases of other picornaviridae are highly divergent on the sequence level, the highly related aphthovirus equine rhinitis A virus may also encode an enzyme that generates GlyGly epitopes, which could be tested using GlyGly epitope detection in infected samples.

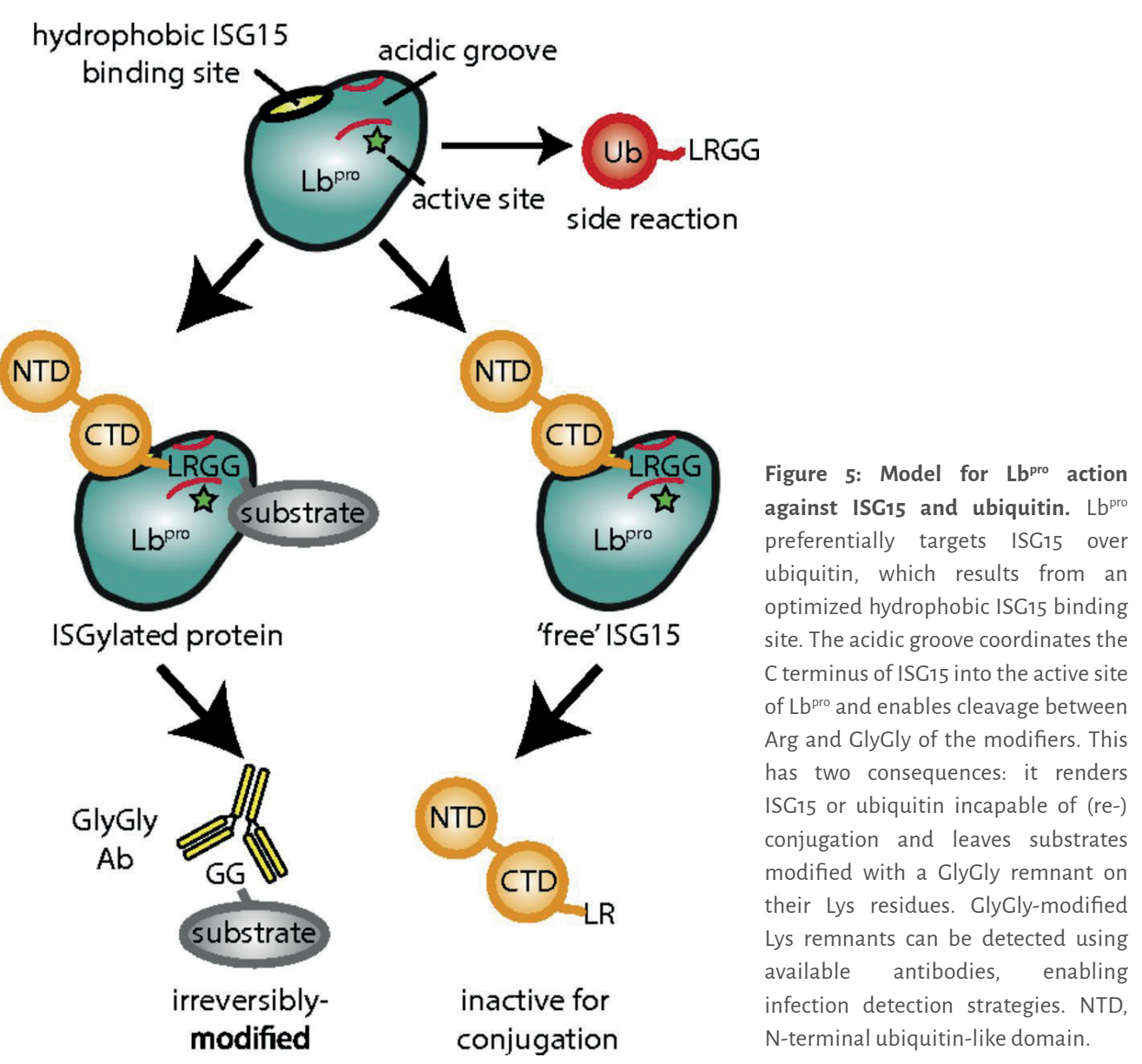

The here identified virus-induced GlyGly remnants on substrate proteins may lead to improvements in the detection of foot-and-mouth disease (FMD). Vaccination is critical to the control FMD outbreaks; however, it is difficult to distinguish vaccinated from infected animals. Current strategies rely on ELISA-based methods to detect antibodies against nonstructural virus proteins in serum. Our findings suggest that GlyGly-modified proteins could also be used in ELISAs to detect antibodies against this epitope. Detection of antibodies against GlyGly modifications signifies enzymatic activity of $\mathrm{Lb}^{\text {pro }}$ that would only be observed after viral infection and hence, distinguish infected from vaccinated animals. This utility of detecting FMDV infection may ease the economic burden imposed by FMD, particularly in developing countries, by providing a previously unrecognized biomarker for its detection.

\section{Methods}

Cloning and Protein Purification. ISG15 and Met1 diubiquitin were cloned into an Histagged expression vector (32). The $\mathrm{Lb}^{\text {pro }}$ vector (23) was expressed and purified according to (33). For the ISG15 ${ }^{\text {CTD }}-\Delta \mathrm{C}$ probe, ISG15 (amino acids 79-154) was cloned in frame into the intein/chitin binding domain PTXB1 vector. ISG15-intein was expressed and purified according to $(17,34)$. SI Methods has expression and purifications procedures for Histagged ISG15 and Met1 diubiquitin.

Biochemistry Assays. ISG15-AMC assays were performed as described previously (18). Ubiquitin/ubiquitin-like TAMRA assays were performed according to (35). ISG15-TAMRA reagent was used to determine Michaelis-Menten kinetics (additional details are in SI Methods). Cleavage assays of pro-ISG15 ${ }^{\text {CTD }}$ and mature ISG $15^{\text {CTD }}$ were performed under the same conditions as the TAMRA assays. ISG15 probe assays were also performed under these conditions. SI Methods has condition details. MS analysis was performed according to (36).

Crystallography. The $\mathrm{Lb}^{\text {pro }} \sim \mathrm{ISG}_{15}{ }^{\mathrm{CTD}}-\Delta \mathrm{C}$ complex was purified by anion chromatography (Resource $\mathrm{Q}$ ) and dialyzed into $50 \mathrm{mM}$ Tris, $\mathrm{pH} 8.0,50 \mathrm{mM} \mathrm{NaCl}, 1 \mathrm{mM}$ EDTA, $5 \mathrm{mM}$ DTT, and $5 \%$ glycerol. After dialysis, the complex was concentrated to $4 \mathrm{mg} / \mathrm{mL}$ and set up at a 1:1 protein:precipitant ratio in a sitting drop vapor diffusion format. Crystals grew in 2.0 $\mathrm{M}$ ammonium sulfate, $0.1 \mathrm{M} \mathrm{Na}$ citrate, $\mathrm{pH} 5.6$, and $0.2 \mathrm{M} \mathrm{K} / \mathrm{Na}$ tartrate and were cryoprotected in mother liquor containing $25 \%$ glycerol. Crystallographic data were collected at the Diamond Light Source synchrotron (Table S1). 


\section{Acknowledgments}

We thank Paul R. Elliott and the beamline scientists at Diamond I-04. Access to Diamond Light Source was supported in part by European Union Seventh Framework Program Infrastructure Grant BIOSTRUCT-X (Contract 283570). This work was supported by The Netherlands Organization for Scientific Research Graduate Program Grant NWO022.004.018 (to L.J.V.), VICI Grants NWO-724.013.002 (to H.O.) and NWO-918.12.628 (to F.J.M.v.K.) from the Netherlands Organization for Scientific Research, the Biotechnology and Biological Sciences Research Council (Pirbright Institute and T.J.T.), Grants P 24038 (to T.S.) and P 28183 (to T.S.) from the Austrian Science Fund, Medical Research Council Grant U105192732 (to D.K.), European Research Council Grants 309756 (to D.K.) and 724804 (to D.K.), and the Lister Institute for Preventive Medicine (D.K.).

\section{References}

1. Tuthill TJ, Groppelli E, Hogle JM, Rowlands DJ. 2010. Picornaviruses. Curr Top Microbiol Immunol.

2. Knight-Jones TJD, Rushton J. 2013. The economic impacts of foot and mouth disease - what are they, how big are they and where do they occur? Prev Vet Med 112:161-73.

3. Grubman MJ, Baxt B. 2004. Foot-and-Mouth Disease. Clin Microbiol Rev.

4. Steinberger J, Skern T. 2014. The leader proteinase of foot-and-mouth disease virus : structure-function relationships in a proteolytic virulence factor 395:1179-1185.

5. Freimanis GL, Di Nardo A, Bankowska K, King DJ, Wadsworth J, Knowles NJ, King DR. 2016. Genomics and outbreaks: Foot and mouth disease. OIE Rev Sci Tech.

6. Liu Y, Zhu Z, Zhang M, Zheng H. 2015. Multifunctional roles of leader protein of foot - and - mouth disease viruses in suppressing host antiviral responses. Vet Res 1-13.

7. Wang D, Fang L, Li P, Sun L, Fan J, Zhang Q, Luo R, Liu X, Li K, Chen H, Chen Z, Xiao S. 2011. The Leader Proteinase of Foot-and-Mouth Disease Virus Negatively Regulates the Type I Interferon Pathway by Acting as a Viral Deubiquitinase. J Virol 85:3758-3766.

8. Isaacson MK, Ploegh HL. 2009. Ubiquitination, Ubiquitin-like Modifiers, and Deubiquitination in Viral Infection. Cell Host Microbe.

9. Heaton SM, Borg NA, Dixit VM. 2016. Ubiquitin in the activation and attenuation of innate antiviral immunity. J Exp Med 213:1-13.

10. Ivashkiv LB, Donlin LT. 2014. Regulation of type I interferon responses. Nat Rev Immunol 14:36-49.

11. Zhao C, Collins MN, Hsiang TY, Krug RM. 2013. Interferon-induced ISG15 pathway: An ongoing virus-host battle. Trends Microbiol.

12. Durfee LA, Lyon N, Seo K, Huibregtse JM. 2010. The ISG15 Conjugation System Broadly Targets Newly Synthesized Proteins: Implications for the Antiviral Function of ISG15. Mol Cell 38:722-32.

13. Zhang X, Bogunovic D, Payelle-Brogard B, Francois-Newton V, Speer SD, Yuan C, Volpi S, Li Z, Sanal O, Mansouri D, Tezcan I, Rice GI, Chen C, Mansouri N, Mahdaviani SA, Itan Y, Boisson B, Okada S, Zeng L, Wang X, Jiang H, Liu W, Han T, Liu D, Ma T, Wang B, Liu M, Liu J-Y, Wang QK, Yalnizoglu D, Radoshevich L, Uzé G, Gros P, Rozenberg F, Zhang S-Y, Jouanguy E, Bustamante J, García-Sastre A, Abel L, Lebon P, Notarangelo LD, Crow YJ, Boisson-Dupuis S, Casanova J-L, Pellegrini S. 2015. Human intracellular ISG15 prevents interferon- $\alpha / \beta$ over-amplification and auto-inflammation. Nature 517:89-93.

14. Bogunovic D, Byun M, Durfee LA, Abhyankar A, Sanal O, Mansouri D, Salem S, Radovanovic I, Grant A V., Adimi P, Mansouri N, Okada S, Bryant VL, Kong XF, Kreins A, Velez MM, Boisson B, Khalilzadeh S, Ozcelik U, Darazam IA, Schoggins JW, Rice CM, Al-Muhsen S, Behr M, Vogt G, Puel A, Bustamante J, Gros P, Huibregtse JM, Abel L, Boisson-Dupuis S, Casanova JL. 2012. Mycobacterial disease and impaired IFN- $\gamma$ immunity in humans with inherited ISG15 deficiency. Science (80-) 337:1684-8.

15. Bhoj VG, Chen ZJ. 2009. Ubiquitylation in innate and adaptive immunity. Nature.

16. Frias-Staheli N, Giannakopoulos N V., Kikkert M, Taylor SL, Bridgen A, Paragas J, Richt JA, Rowland RR, Schmaliohn CS, Lenschow DI, Snijder EJ García-Sastre A, Virgin IV HW, 2007. Ovarian Tumor DomainContaining Viral Proteases Evade Ubiquitin- and ISG15-Dependent Innate Immune Responses. Cell Host Microbe.

17. Pruneda JN, Durkin CH, Geurink PP, Ovaa H, Santhanam B, Holden DW, Komander D. 2016. The Molecular Basis for Ubiquitin and Ubiquitin-like Specificities in Bacterial Effector Proteases. Mol Cell.

18. Akutsu M, Ye Y, Virdee S, Chin JW, Komander D. 2011. Molecular basis for ubiquitin and ISG15 crossreactivity in viral ovarian tumor domains. Proc Natl Acad Sci U S A.

19. James TW, Frias-Staheli N, Bacik JP, Levingston Macleod JM, Khajehpour M, García-Sastre A, Mark BL. 2011. Structural basis for the removal of ubiquitin and interferon-stimulated gene 15 by a viral ovarian tumor domain-containing protease. Proc Natl Acad Sci U S A. 
20. Basters A, Geurink PP, El Oualid F, Ketscher L, Casutt MS, Krause E, Ovaa H, Knobeloch KP, Fritz G. 2014. Molecular characterization of ubiquitin-specific protease 18 reveals substrate specificity for interferonstimulated gene 15. FEBS $\mathrm{J}$.

21. van Tilburg GBA, Elhebieshy AF, Ovaa H. 2016. Synthetic and semi-synthetic strategies to study ubiquitin signaling. Curr Opin Struct Biol.

22. Basters A, Geurink PP, Röcker A, Witting KF, Tadayon R, Hess S, Semrau MS, Storici P, Ovaa H, Knobeloch KP, Fritz G. 2017. Structural basis of the specificity of USP18 toward ISG15. Nat Struct Mol Biol.

23. Guarné A, Tormo J, Kirchweger R, Pfistermueller D, Fita I, Skern T. 1998. Structure of the foot-and-mouth disease virus leader protease: A papain-like fold adapted for self-processing and eIF4G recognition. EMBO J 17:7469-7479.

24. Komander D, Clague MJ, Urbé S. 2009. Breaking the chains: Structure and function of the deubiquitinases. Nat Rev Mol Cell Biol.

25. Deaton MK, Dzimianski J V., Daczkowski CM, Whitney GK, Mank NJ, Parham MM, Bergeron E, Pegan SD. 2016. Biochemical and Structural Insights into the Preference of Nairoviral DeISGylases for InterferonStimulated Gene Product 15 Originating from Certain Species. J Virol.

26. Keusekotten K, Elliott PR, Glockner L, Fiil BK, Damgaard RB, Kulathu Y, Wauer T, Hospenthal MK, GyrdHansen M, Krappmann D, Hofmann K, Komander D. 2013. OTULIN antagonizes LUBAC signaling by specifically hydrolyzing met1-linked polyubiquitin. Cell.

27. Kim W, Bennett EJ, Huttlin EL, Guo A, Li J, Possemato A, Sowa ME, Rad R, Rush J, Comb MJ, Harper JW, Gygi SP. 2011. Systematic and quantitative assessment of the ubiquitin-modified proteome. Mol Cell.

28. Wagner SA, Beli P, Weinert BT, Nielsen ML, Cox J, Mann M, Choudhary C. 2011. A Proteome-wide, Quantitative Survey of In Vivo Ubiquitylation Sites Reveals Widespread Regulatory Roles. Mol Cell Proteomics.

29. Rabouw HH, Langereis MA, Knaap RCM, Dalebout TJ, Canton J, Sola I, Enjuanes L, Bredenbeek PJ, Kikkert M, de Groot RJ, van Kuppeveld FJM. 2016. Middle East Respiratory Coronavirus Accessory Protein 4a Inhibits PKR-Mediated Antiviral Stress Responses. PLoS Pathog 12:1005982.

30. Choy A, Dancourt J, Mugo B, O'Connor TJ, Isberg RR, Melia TJ, Roy CR. 2012. The Legionella effector RavZ inhibits host autophagy through irreversible Atg8 deconjugation. Science (80-).

31. Chinsangaram J, Mason PW, Grubman MJ. 1998. Protection of swine by live and inactivated vaccines prepared from a leader proteinase-deficient serotype A12 foot-and-mouth disease virus. Vaccine.

32. Berrow NS, Alderton D, Sainsbury S, Nettleship J, Assenberg R, Rahman N, Stuart DI, Owens RJ. 2007. A versatile ligation-independent cloning method suitable for high-throughput expression screening applications. Nucleic Acids Res.

33. Aumayr M, Fedosyuk S, Ruzicska K, Sousa-Blin C, Kontaxis G, Skern T. 2015. NMR analysis of the interaction of picornaviral proteinases $\mathrm{Lb}$ and $2 \mathrm{~A}$ with their substrate eukaryotic initiation factor 4GII. Protein Sci 24:1979-1996.

34. Ekkebus R, Van Kasteren SI, Kulathu Y, Scholten A, Berlin I, Geurink PP, De Jong A, Goerdayal S, Neefies , Heck AJR, Komander D, Ovaa H. 2013. On terminal alkynes that can react with active-site cysteine nucleophiles in proteases. J Am Chem Soc.

35. Geurink PP, El Oualid F, Jonker A, Hameed DS, Ovaa H. 2012. A General Chemical Ligation Approach Towards Isopeptide-Linked Ubiquitin and Ubiquitin-Like Assay Reagents. ChemBioChem 13:293-7.

6. Wauer T, Swatek KN, Wagstaff JL, Gladkova C, Pruneda JN, Michel MA, Gersch M, Johnson CM, Freund SM, Komander D. 2015. Ubiquitin Ser65 phosphorylation affects ubiquitin structure, chain assembly and hydrolysis. EMBO J.

\section{References exclusively used in supplementary information}

37. Battye TGG, Kontogiannis L, Johnson O, Powell HR, Leslie AGW. 2011. iMOSFLM: A new graphical interface for diffraction-image processing with MOSFLM. Acta Crystallogr Sect D Biol Crystallogr.

38. Evans PR, Murshudov GN. 2013. How good are my data and what is the resolution? Acta Crystallogr Sect D Biol Crystallogr.

39. McCoy AJ, Grosse-Kunstleve RW, Adams PD, Winn MD, Storoni LC, Read RJ. 2007. Phaser crystallographic software. J Appl Crystallogr.

40. Emsley P, Lohkamp B, Scott WG, Cowtan K. 2010. Features and development of Coot. Acta Crystallogr Sect D Biol Crystallogr.

41. Adams PD, Afonine P V., Bunkóczi G, Chen VB, Echols N, Headd JJ, Hung LW, Jain S, Kapral GJ, Grosse Kunstleve RW, McCoy AJ, Moriarty NW, Oeffner RD, Read RJ, Richardson DC, Richardson JS, Terwilliger TC, Zwart PH. 2011. The Phenix software for automated determination of macromolecular structures. Methods.

42. Stivala A, Wybrow M, Wirth A, Whisstock JC, Stuckey PJ. 2011. Automatic generation of protein structure cartoons with pro-origami. Bioinformatics.

43. Cencic R, Mayer C, Juliano MA, Juliano L, Konrat R, Kontaxis G, Skern T. 2007. Investigating the Substrate Specificity and Oligomerisation of the Leader Protease of Foot and Mouth Disease Virus using NMR. J Mol Biol. 


\section{Supplementary information}

A

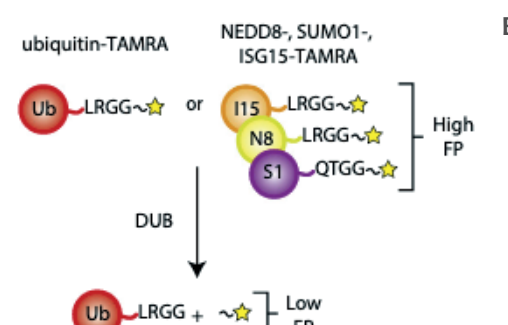

c
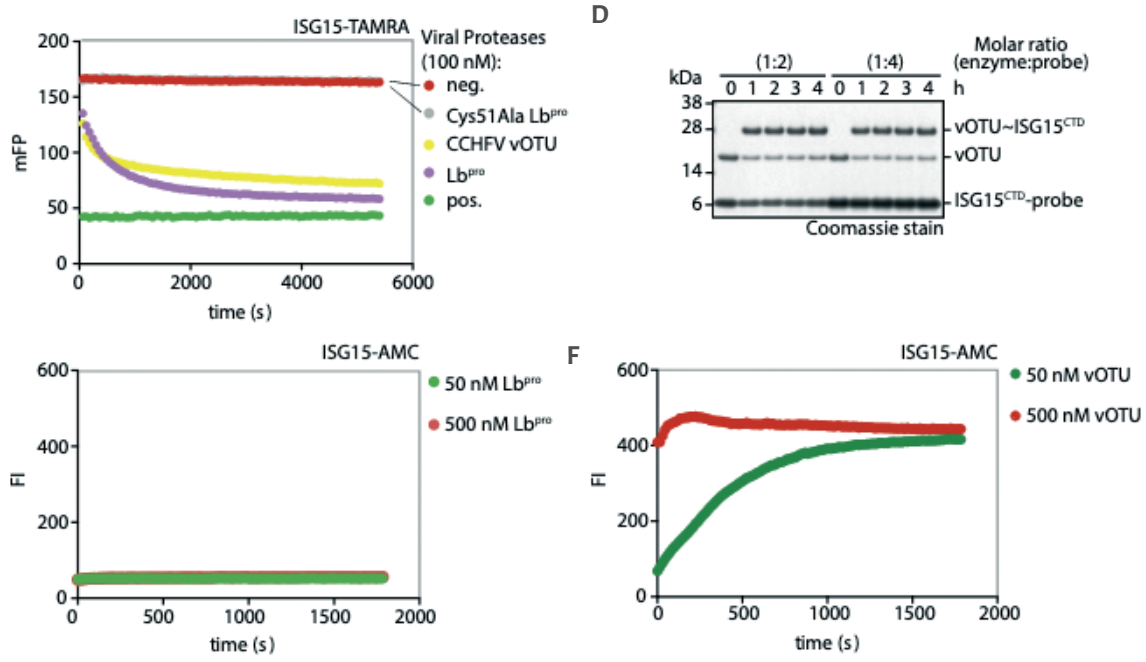

$c$

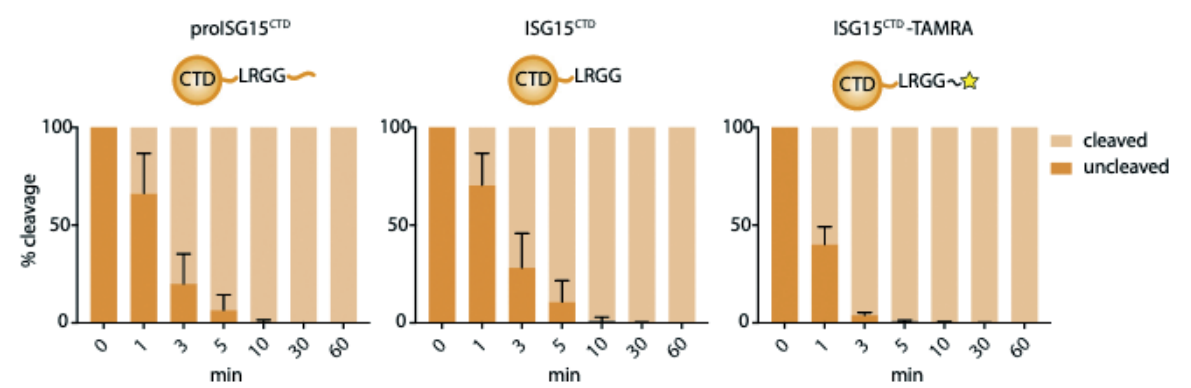

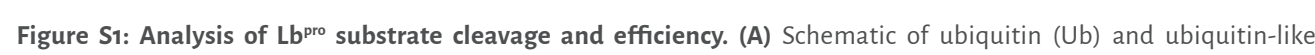
FP cleavage assays using TAMRA-labeled substrates. (B) Ubiquitin-TAMRA cleavage by $L b^{\text {pro }}$ at increased enzyme concentrations. (C) ISG15-TAMRA cleavage assays by viral proteases. Viral proteases include $L b^{\text {pro }}$, L $b^{\text {pro }} C 51 A$, and CCHFV vOTU. Positive (+) and negative (-) controls correspond to fully cleaved and uncleaved TAMRA reagents, respectively.(D)

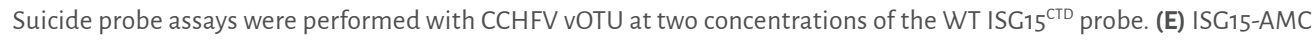
cleavage assays with Lb ${ }^{\text {pro }}$. Cleavage of ISG15-AMC was measured by fluorescence intensity (FI). Lb pro cleaves ISG15-AMC, but the produced ClyCly-AMC remains nonfluorescent (Fig. S2A). (F) ISG15-AMC cleavage assays as in E with CCHFV vOTU. In contrast to Lb pror, robust cleavage of ISG15-AMC by CCHFV vOTU was detected. (G) Comparison of proISG15 $5^{\text {CTD }}$, mature ISG15 ${ }^{\mathrm{CT} D}$, and ISG15 $5^{\mathrm{CT} D}$-TAMRA (isopeptide-linked ISG15) cleavage rates using intact MS. All assays were performed in triplicate. $\mathrm{mFP}$, measured fluorescence polarization.
A ISG15-AMC

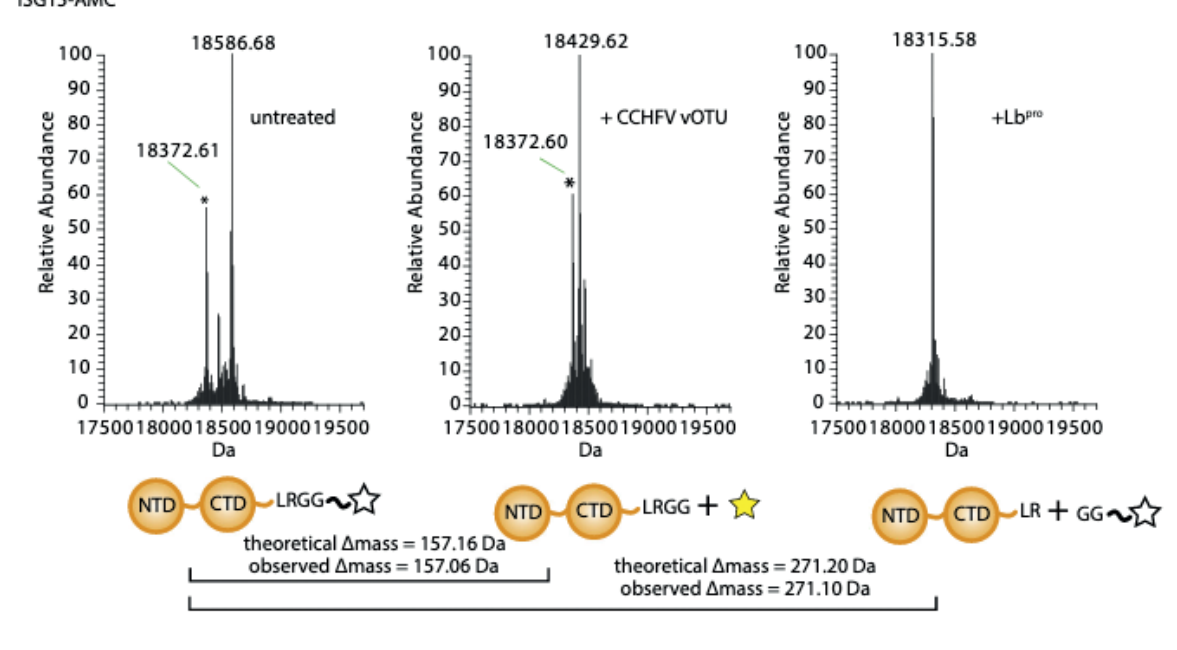

B
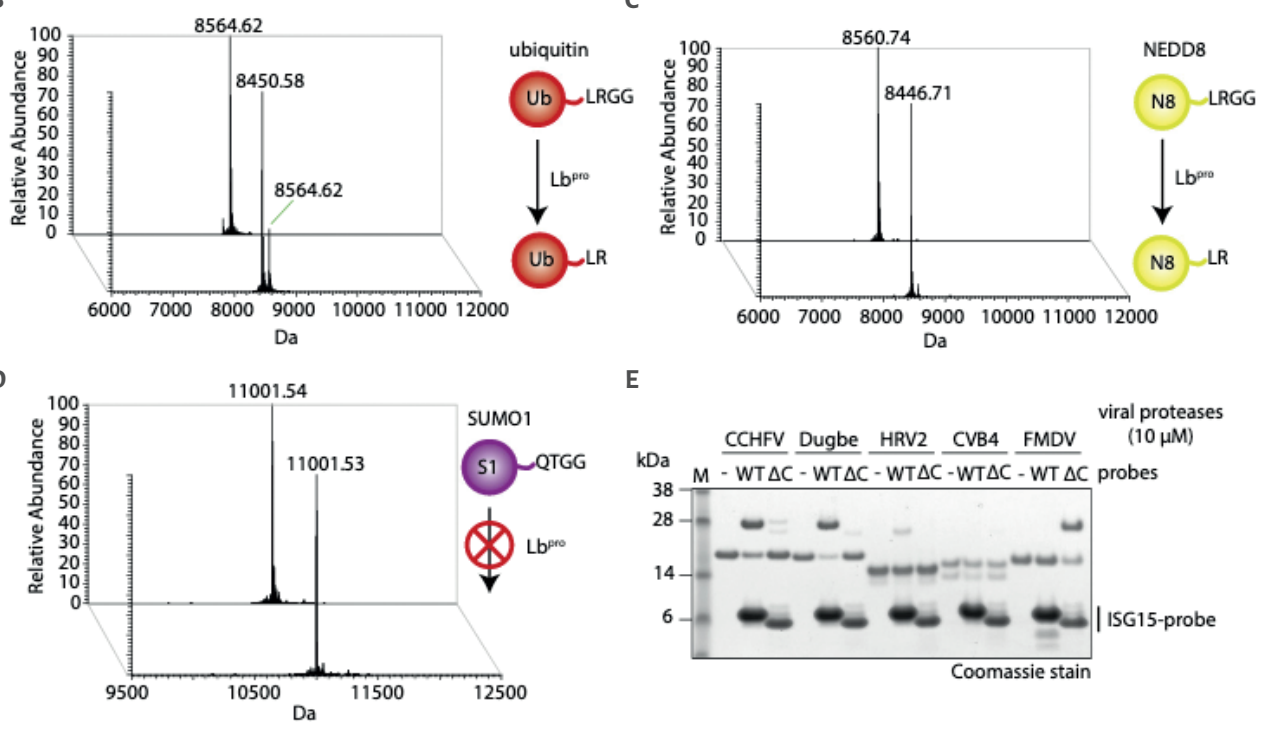

Figure S2: Characterization of Lb ionization MS without and with incubation of CCHFV VOTU and Lbro. The deconvoluted spectrum is shown, and a diagram depicts the theoretical and observed $\Delta$ masses of cleavage. "Contaminant of ISG15-AMC synthesis; this mass corresponds to ISG15 lacking the most C-terminal Cly residue. (B) Ubiquitin, (C) NEDD8, and (D) SUMO1 were analyzed by electrospray ionization intact MS before and after incubation with $L b^{\text {pro }}$. The deconvoluted spectrum is shown. A diagram shows the ubiquitin and NEDD8 cleavage site. SUMO1 cleavage was not observed. (E) Reactivity of viral proteases with WT and ISG15 $1 \mathrm{CT}^{\mathrm{T}}-\Delta \mathrm{C}$ suicide probes. The viral proteases include CCHFV vOTU, Dugbe protease, human rhinovirus serotype $22 A^{\text {pro }}\left(H R V 22 A^{\text {pro }}\right)$, coxsackievirus serotype B4 $2 A$ protease (CVB4 2A $A^{\text {pro }}$ ), and FMDV Lb ${ }^{\text {pro }}$. Reactions were incubated at $37{ }^{\circ} \mathrm{C}$ and subsequently resolved by Coomassie-stained SDS-PACE. All assays were performed in triplicate. NTD, N-terminal ubiquitin-like domain. 

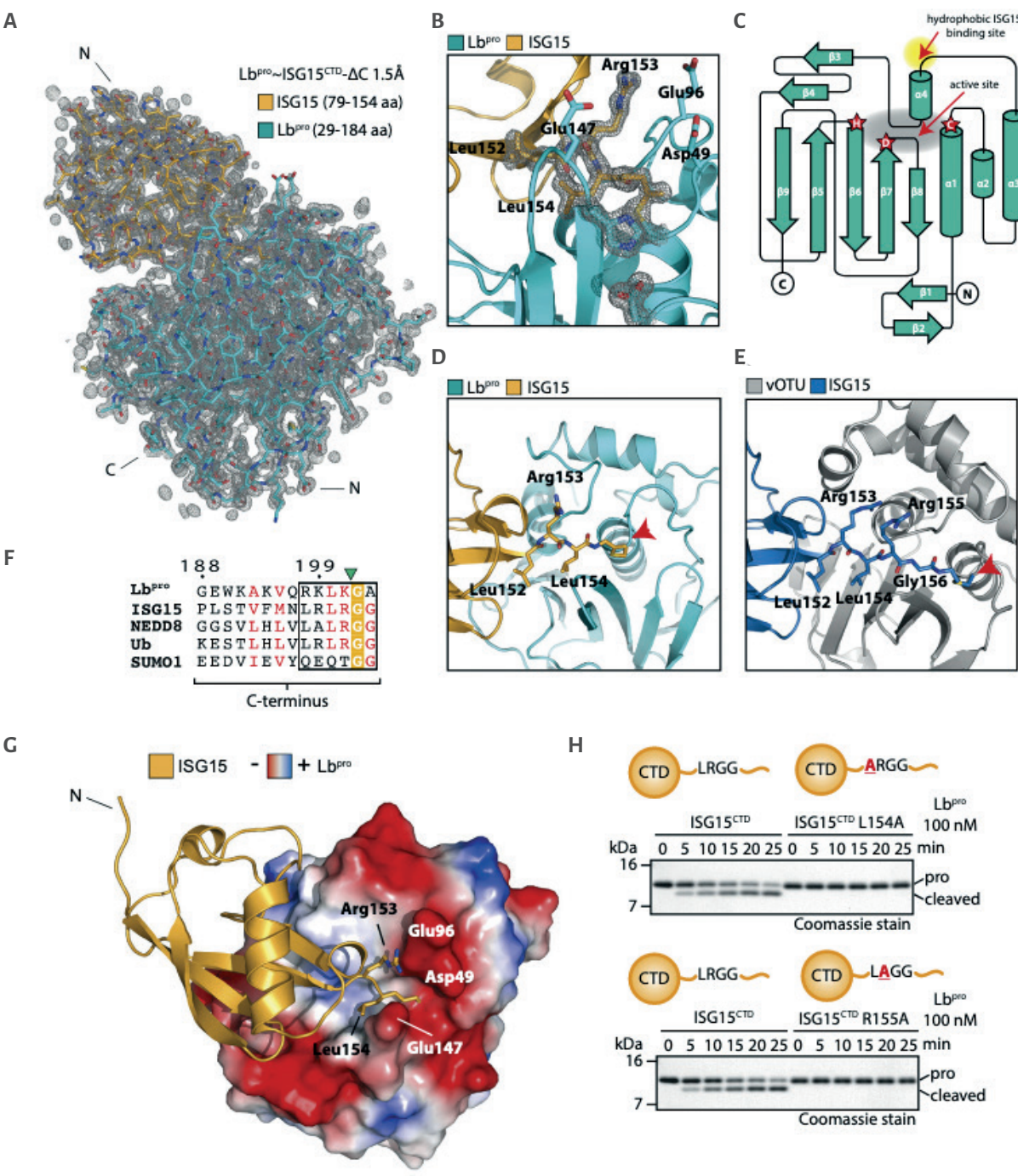

(CTD $L R G G \sim$ (TD) $A R G G$

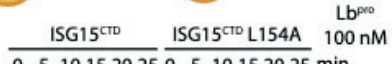
Da 05161520250510152025 min Coomassie stain (CTD LRGG CTD LAGG -

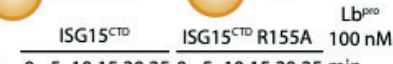

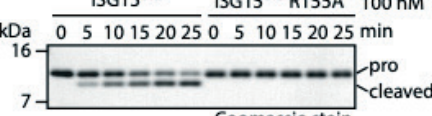

Figure $S_{3}$ : Structural analysis of the $L b^{\text {pro }} \sim I S G 15^{\text {CTD }}-\Delta C$ structure. (A) Residues of the $L b^{\text {pro }} \sim I S G 15^{C^{C T D}}-\Delta C$ crystal structure are shown in stick representation for the complex in the asymmetric unit covered by the $1.5-\AA$ ¿ $2|\mathrm{Fo}|-|\mathrm{Fc}|$ electron density

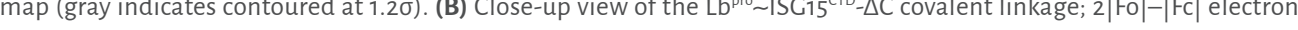
density is shown for the C terminus of ISG15 (Leu152, Arg153, Leu154, propargyl warhead), and thecatalytic triad of the ISG15 hydrophobic binding pocket is indicated with a yellow circle. (D) Comparison of $L b^{\text {pro }} \sim \mathrm{SG} 15^{\mathrm{CTD}}-\Delta \mathrm{C}$ with (E) CCHFV VOTU ISG15 complex (PDB ID code 3phx). Close-up view of the covalently modified active site Cys residues of $\mathrm{Lb}^{\mathrm{pro}} \sim \mid \mathrm{SG} 15^{\mathrm{CTD}}-\triangle \mathrm{C}$ and VOTU $\sim \mathrm{SG} 15$ (red arrows). (F) Sequence alignment of the $\mathrm{C}$-terminal residues of $\mathrm{L} \mathrm{b}^{\text {pro self }}$ secleavage to ubiquitin (Ub) and ubiquitin-like proteins (ISG15, NEDD8, SUMO1). A green arrow denotes the site of Lb ${ }^{\text {pro }}$ selfcleavage, and amino acid numbers are labeled according to $L b^{\text {pro }}$.(C) lonic interactions of $L b^{\text {pro }}$ with the $C$ terminus of ISC15. Electrostatic potential of $L b^{\text {pro }}$ is shown in surface representation. Red represents negative electrostatic potential, while blue is positive. (H) prolSG15 $15^{\mathrm{Cr} D}$ cleavage assays as in Fig. 1D. Point mutants in the ISG15 C terminus (L154A and R155A) were incubated with Lb pro $^{\text {pro }}$ resolved by SDS-PACE, and stained with Coomassie. All assays were performed in triplicate.
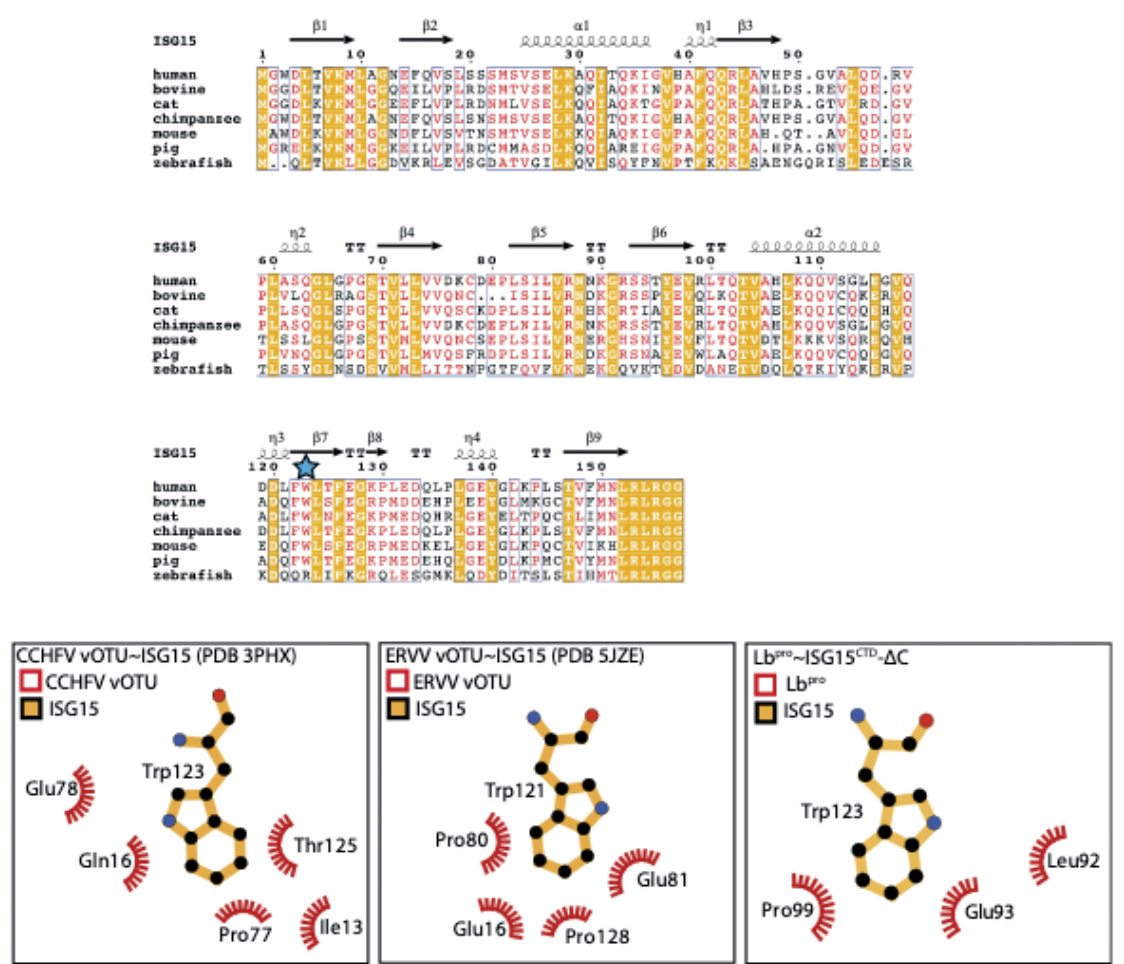

c

D (10)

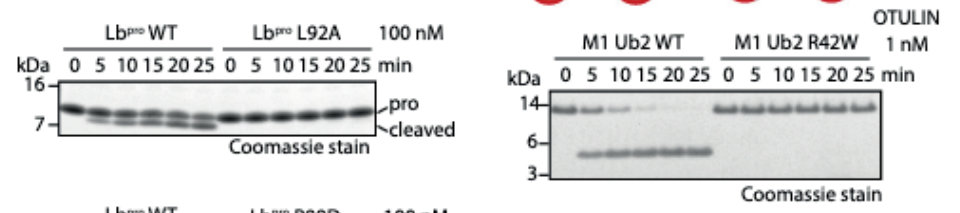

kDa $\frac{\text { LbmowT }}{0.510152025} \frac{\text { Lber P99D }}{0.510152025} 100 \mathrm{nM}$

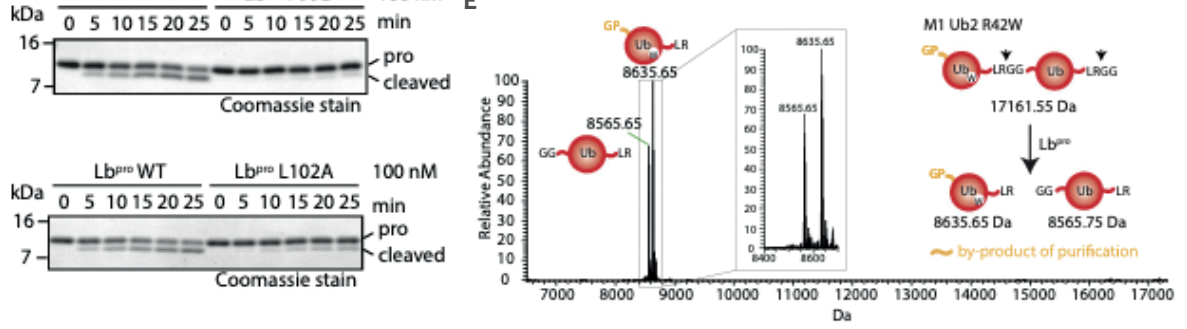

Figure S4: Characterization of Lb ${ }^{\text {pro }}$ ISG15 specificity. (A) Sequence alignment of ISG15 across multiple species. Trp123 is highlighted with a blue star and isconserved across vertebrates, except zebrafish. Another important area of sequence conservation is the C terminus. (B) LICPLOT representation of Trp123 (Trp121 of mice) ISG15 interactions with L b $b^{\text {pro }}$ and viral delSCylases, including CCHFV VOTU [PDB ID code 3 phx] and Erve (ERVV) vOTU [PDB ID code 5jze].Trp123 is shown in orange, and residues that form hydrophobic interactions with this residue are shown as red fans. (C) prolSG15 $15^{\text {CD }}$ (amino acids 79-165) cleavage assays with mutations in the hydrophobic patch that make contact with Trp123 of ISC15. (D) OTULIN cleavage of Met1 diubiquitin substrates (compare with Fig. 3E). (E) Intact MS analysis of Met1 diubiquitin cleavage assays with $\mathrm{R} 42 \mathrm{~W}$ mutation in the distal ubiquitin (Ub). All assays were performed in triplicate. 


$$
\begin{gathered}
\text { hydrophobic ISG } 15 \\
\text { binding site }
\end{gathered}
$$$$
\text { [92A, P99A, L102A point mutants }
$$

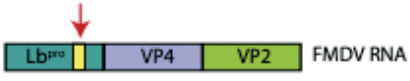
$\downarrow \begin{gathered}\text { in vitro translation } \\ \text { [35S]methionine }\end{gathered}$

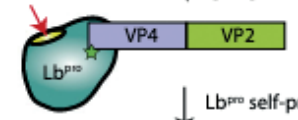

$\downarrow$ Lbrow self-processing

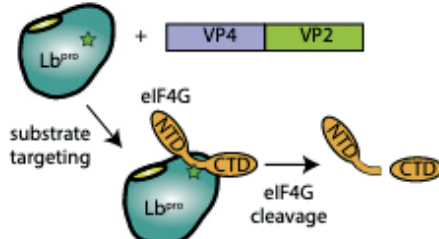

elF4G

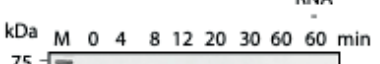
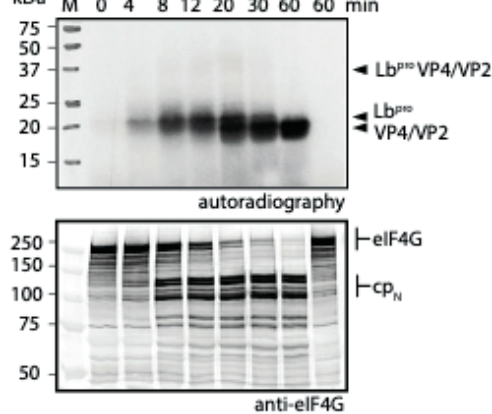

LbPrw wTV4NP2

kDa $\begin{array}{llllll}M & 0 & 8 & 12 & 20306060 \\ 75\end{array}$

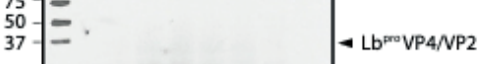

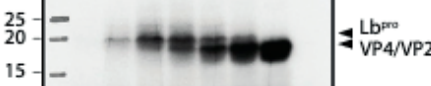

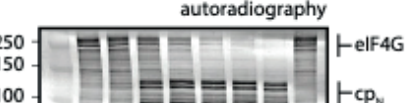

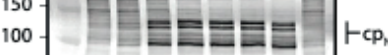

75 . 느므트트트트틀

anti-elF4G

Lb" 999 A VP4NP2 2 RNA

KDa M 04481220306060 min
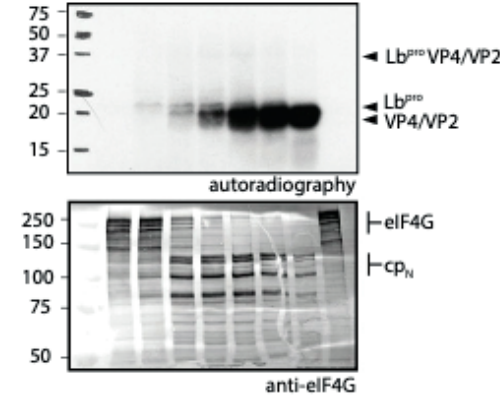

LbF"LLO2A VPANP2 RNA

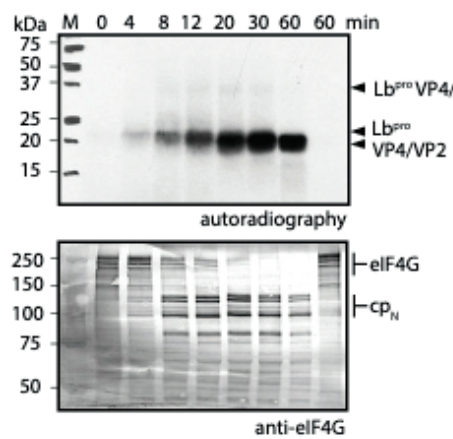

Figure S5: Mutations in the hydrophobic ISG15 binding site of Lb ${ }^{\text {pro }}$ do not impact self-processing and elF4G cleavage. (A) Schematic of in vitro translation assays. The indicated RNA was translated in rabbit reticulocyte lysates (RRLs) containing [35S] methionine. On translation, L $b^{\text {pro }}$ undergoes self-processing, removing itself from the nascent polyprotein ( $\mathrm{VP}_{4} / \mathrm{PP}^{2}$ ). Liberated $L b^{\text {pro }}$ then targets elF4C for cleavage. (B)WT L L ${ }^{\text {pro }}$ and (C) L $b^{\text {pro }}$ mutant (L92A, P99A, andL102A) VP4/VP2 RNA were incubated in [35S] methionine containing RRLs. Time courses of Lbpro self-processing and elF4G cleavage were detected by autoradiography and anti-elF4G antibody, respectively. NTD, N-terminal ubiquitin-like domain.
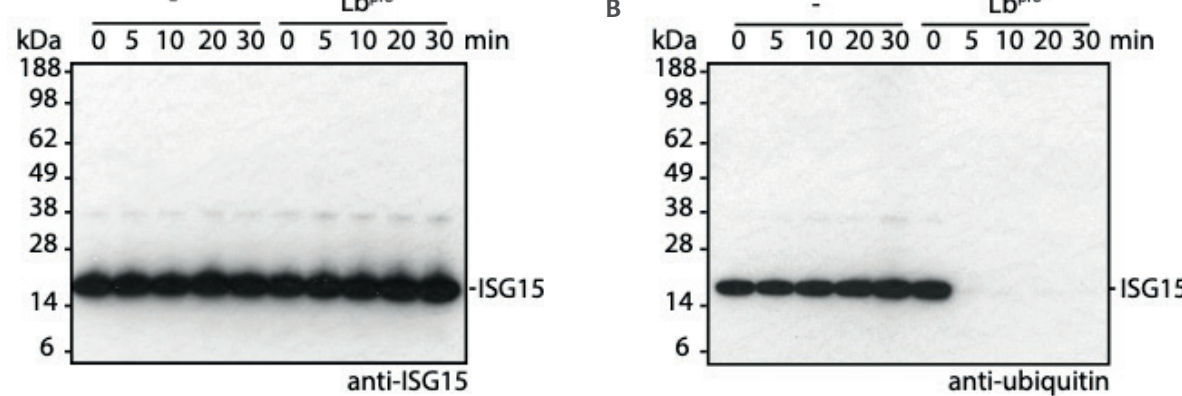

anti-ubiquitin

C HeLa, FLAG-ISG15

E2: UBE2L6,
E3: HERCS
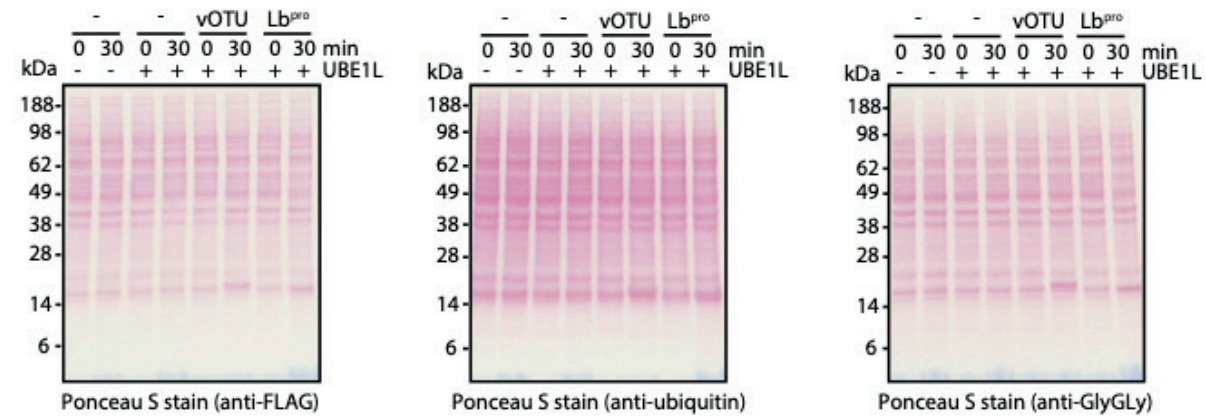

D HeLa

FMDV Lb L
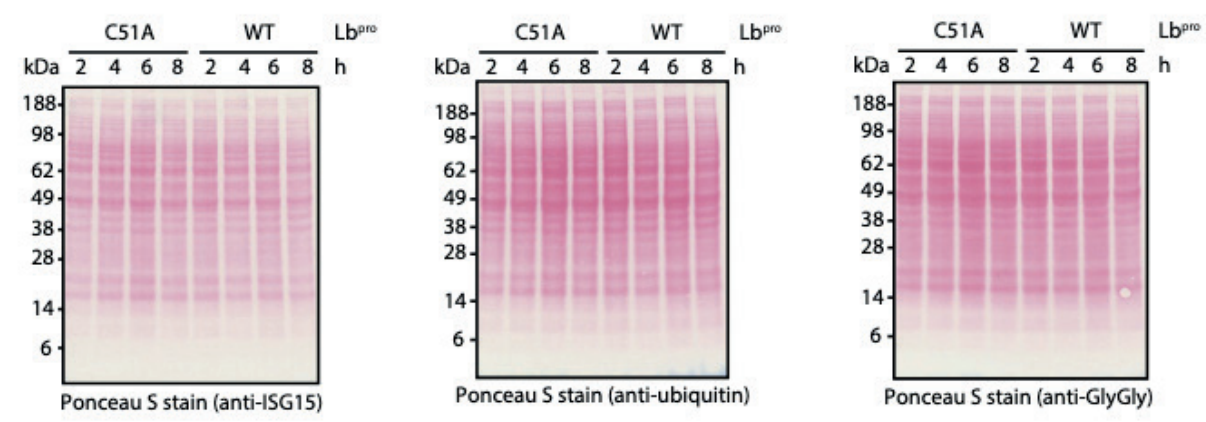

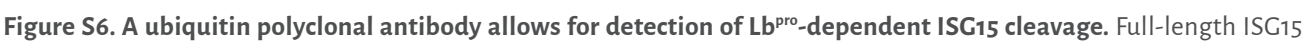
(1-157 amino acids) was incubated with $L b^{\text {pro }}$ in a standard cleavage assay. The indicated reactions and time points were analyzed by Western blotting using (A) anti-ISG15 antibody (H-150; Santa CruzInc.) and (B) anti-ubiquitin antibody (Millipore). Detection of ISG15 cleavage by $\mathrm{Lb}^{\text {pro }}$ can be visualized using the polyclonal anti-ubiquitin antibody, which cross-reacts with the conserved $C$ terminus of ISG15. On cleavage of the $C$ terminus by L $b^{\text {pro }}$, the antibody no longer recognizes ISG15. (C) Ponceau S-stained blots from Fig. 4A.(D) Ponceau S-stained blots from Fig. 4B. 


\begin{tabular}{|c|c|}
\hline & $L b^{\text {pro }} \sim I S G 15-\Delta C$ \\
\hline \multicolumn{2}{|l|}{ Data collection } \\
\hline \multicolumn{2}{|l|}{ Cell dimensions } \\
\hline $\begin{array}{l}a, b, c, \AA \\
\alpha, \beta, \gamma,{ }^{\circ}\end{array}$ & $\begin{array}{c}114.19,40.35,58.05 \\
90,91.82,90\end{array}$ \\
\hline $\begin{array}{l}\text { Resolution, } \AA \\
\mathrm{R}_{\text {merge }} \\
\| / \sigma \mid\end{array}$ & $\begin{array}{c}58.02-1.50(1.53-1.50) \\
0.044(0.489) \\
13.4(2.2)\end{array}$ \\
\hline $\mathrm{CC}_{1 / 2}$ & $\begin{array}{c}13.4(2.2) \\
0.999(0.866)\end{array}$ \\
\hline Completeness, \% & $99.2(98.4)$ \\
\hline Redundancy & $4.2(3.9)$ \\
\hline \multicolumn{2}{|l|}{ Refinement } \\
\hline Resolution, $\AA$ & $58.02-1.50$ \\
\hline Unique reflections & 42,227 \\
\hline $\begin{array}{l}R_{\text {work }} / R_{\text {free }} \\
\text { No. of atoms }\end{array}$ & $16.7 / 18.6$ \\
\hline Protein & 1,881 \\
\hline Ligand/ion & 49 \\
\hline Water & 213 \\
\hline \multicolumn{2}{|l|}{ B factors } \\
\hline Protein & 24.2 \\
\hline Ligand/ion & 60.8 \\
\hline Water & 36.3 \\
\hline \multicolumn{2}{|l|}{ rmsds } \\
\hline Bond lengths, $\AA$ & 0.020 \\
\hline Bond angles, ${ }^{\circ}$ & 1.43 \\
\hline
\end{tabular}

Table S1: Data collection and refinement statistics. Values in parentheses are for highest-resolution shell.

\section{SI Methods}

Cloning and Site-Directed Mutagenesis.The expression vectorpET11d/FMDV (23) was used as a template for all site-directed $\mathrm{Lb}^{\text {pro }}$ mutants (amino acids 29-195). Human ISG15 (Cys78Ser) and Met1 diubiquitin constructs were cloned into the pOPIN-BHis-tagged expression vector (32). A codon-optimized ISG15 was obtained from GeneArt Gene Synthesis (Invitrogen) and cloned in frame to the intein/chitin binding domain pTXB1 vector (NewEngland BioLabs) using conventional cloning methods. Site-directed mutagenesis was performed according to the QuikChange method. Plasmids for cell transfection assays (FLAG-ISG15, UBE1L,UBE2L6, and HERC5 of human origin) were cloned into the pcDNA3.1 mammalian expression vector.
Protein Expression and Purification. Lb ${ }^{\text {pro }}$ was expressed and purified according to (33). His-tagged ISG15 and Met1 diubiquitin were expressed in Escherichia coli Rosetta2 (DE3) pLacI (Novagen). Cultures were grown to OD600 (0.6-0.8) and induced with $0.2 \mathrm{mM}$ Isopropyl $\beta$-D-1-thiogalactopyranoside for $16 \mathrm{~h}$ at1 ${ }^{\circ} \mathrm{C}$. Cell pellets were resuspended in $30 \mathrm{~mL}$ of lysis buffer (50 mM Tris, $\mathrm{pH} 7.4,150 \mathrm{mM} \mathrm{NaCl}, 2 \mathrm{mM} \beta$-mercaptoethanol) and frozen at $-80^{\circ} \mathrm{C}$. Thawed pellets were supplemented with an EDTA-free Complete Protease Inhibitor tablet (Roche), $1 \mathrm{mg} / \mathrm{mL}$ lysozyme, and $0.1 \mathrm{mg} / \mathrm{mL}$ DNaseI and incubated on ice for $10 \mathrm{~min}$.The cell suspension was sonicated, and cell debris was cleared by centrifugation at 50,000 $\mathrm{g}$ for $30 \mathrm{~min}$. The cleared lysate was incubated with TALON Superflow resin (GE Healthcare) for 10 min and washed with $1 \mathrm{~L}$ of lysis buffer by gravity flow. Protein was eluted with lysis buffer supplemented with $250 \mathrm{mM}$ imidazole. Purified proteins were dialyzed for $16 \mathrm{~h}$ into lysis buffer to remove excess imidazole. ISG15-intein protein was expressed identically to His-tagged proteins but purified with chitin resin (New England BioLabs) as described previously $(17,34)$.

AMC Fluorescence Measurements. ISG15-AMC cleavage assays were performed as described previously (18) with minor modifications. In each reaction, $10 \mu \mathrm{L}$ of $2 \mu \mathrm{M}$ ISG15AMC ( $2 \times$ concentration) was mixed with $10 \mu \mathrm{L}$ enzyme ( $2 \times$ concentration) inAMC assay buffer ( $50 \mathrm{mM}$ Tris, $\mathrm{pH} 8.0,10 \mathrm{mM}$ DTT). The reactions were monitored using $\lambda$ ex $=350 \mathrm{~nm}$ and $\lambda \mathrm{em}=450 \mathrm{~nm}$.

Fluorescence Polarization Assays. All ubiquitin/ubiquitin-like TAMRA assays were performed according to (35). Briefly, enzymes and substrates were diluted in dilution buffer (50 mM Tris, pH 8.0, $10 \mathrm{mM}$ DTT). Substrate concentrations remained constant at $150 \mathrm{nM}$, while enzyme concentrations varied based on cleavage efficiency. Cleavage assays were performed in a black 384-wellplate (Corning), and fluorescence polarization (FP) measurements were made with a $550 \mathrm{~nm}$ excitation filter and a $590 \mathrm{~nm}$ emission filter using a Pherastar plate reader (BMG Labtech). All measurements were compared with substrate only (negative) and $25 \mathrm{nM}$ KG-TAMRA peptide (positive) reference standards.

Kinetics. ISG15-TAMRA was used as a substrate for determination of $\mathrm{Lb}^{\text {pro }}$ MichaelisMenten kinetics. The change in FP on Lb $b^{\text {pro }}$ hydrolysis was measured in a black 384-well plate using a PheraStar plate reader (BMG Labtech): $\lambda$ ex $=550 \mathrm{~nm}$ and $\lambda \mathrm{em}=590 \mathrm{~nm}$. Reactions were incubated at $25^{\circ} \mathrm{C}$ in a total volume of $20 \mu \mathrm{L}$ in FP kinetics buffer $(25 \mathrm{mM}$ Tris, $\mathrm{pH} 8.5$, $2 \mathrm{mM} \mathrm{DTT}, 0.1 \mathrm{mg} / \mathrm{mL} \mathrm{BSA}$ ). In each well, $10 \mu \mathrm{L}$ ISG15-TAMRA ( $2 \times$ finalconcentration) was mixed with $10 \mu \mathrm{L}$ of $40 \mathrm{nM}$ enzyme ( $2 \times$ finalconcentration). The ISG15-TAMRA concentration was varied from 0.1 to $20 \mu \mathrm{M}$. All reactions were performed in triplicate and in parallel. The change in FP was measured in the linear range over a period of $0-7 \mathrm{~min}$, with readings recorded every $5 \mathrm{~s}$. The FP measurements were plotted over time, and initial velocities of 
ISG15-TAMRA cleavage were determined. Michaelis-Menten parameters were calculated using Prism 6 GraphPad Software.

proISG15 Cleavage Assays. The proforms of ISG15 full-length (1-165) or the CTD (79-165) were used in all cleavage assays. The concentration of proISG15 was held constant at 10 $\mu \mathrm{M}$ and incubated at $37^{\circ} \mathrm{C}$ in all cleavage assays. Reactions were quenched through the addition of lithium dodecyl sulfate (LDS) samplebuffer at the indicated time points. Samples were resolved on $10-20 \%$ Tricine gels (Invitrogen) and stained with Instant BlueSafeStain (Expedeon).

Intact MS. Cleavage assays were performed similarly to the proISG15 cleavage assays described above. In ubiquitin (1-76 amino acids), NEDD8 (1-76 amino acids), and SUMO1 (1-97 amino acids) cleavage assays, the concentration of $L^{\text {pro }}$ was increased to $1 \mu \mathrm{M}$ to promote cleavage of these non-preferred substrates. Unless stated otherwise, reactions were incubated at $37{ }^{\circ} \mathrm{C}$ for $\mathrm{ih}$ and quenched with $50 \%$ acetonitrile and $0.1 \%$ formic acid. Substrates were diluted to $1 \mathrm{pmol} / \mu \mathrm{L}$ before intact MS analysis. Samples were injected at a flow rate of $5 \mu \mathrm{L} / \mathrm{min}$ and ionized using a heated electrospray ionization (HESI-II) source before being analyzed by an Orbitrap mass analyzer (QExactive). Ionization, data collection, and deconvolution of rawfiles were performed according to (36).

ISG15 Suicide Probe Assays. ISG15 probe assays were performed with 20 or $40 \mu \mathrm{M}$ ISG15 probes with $10 \mu \mathrm{M} \mathrm{Lb} b^{\text {pro }}$ or vOTU at $37^{\circ} \mathrm{C}$ in probe assay buffer (100 mM Tris, $\mathrm{pH} 8.0,10$ $\mathrm{mM}$ DTT). The reactions were quenched in LDS sample buffer at the specified time points and resolved on a $4-12 \%$ Bis-Tris gel before being visualized with Instant Blue SafeStain (Expedeon).

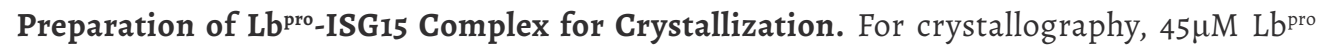
was mixed with $175 \mu \mathrm{M}$ CTD-ISG15- $\Delta$ Cprobe in a total volume of $1 \mathrm{~mL}$. The reaction was incubated for $4 \mathrm{~h}$ at $37{ }^{\circ} \mathrm{C}$. Immediately afterward, the reaction was placed on ice.The resulting complex was purified by strong anion chromatogra-phy (Resource $\mathrm{Q}$ ) at $\mathrm{pH}$ 8.0 using a linear salt gradient $(0-500 \mathrm{mMNaCl})$. The reacted complex was pooled and dialyzed into $50 \mathrm{mM}$ Tris, pH 8.0, $50 \mathrm{mM} \mathrm{NaCl}$, $1 \mathrm{mM}$ EDTA, $5 \mathrm{mM}$ DTT, and $5 \%$ glycerol. After dialysis, the complex was concentrated to $4 \mathrm{mg} / \mathrm{mL}$ using a Vivaspin 500 3K MWCO concentrator and used for crystallization screening. The purity of the complex was assessed by a Coomassie-stained SDS-PAGE gel and estimated to be $>95 \%$ pure.

Crystallization. Initial hits were obtained from a commercial screen (Hampton Research) in a sitting drop vapor diffusion format. Lb ${ }^{\text {pro }} \sim$ ISG $15-\Delta C$ complex was set up at a 1:1 protein:precipitant ratio. Crystals grew in $2.0 \mathrm{M}$ ammonium sulfate, $0.1 \mathrm{M} \mathrm{Na}$ citrate $\mathrm{pH}$
5.6 , and $0.2 \mathrm{M} \mathrm{K} / \mathrm{Na}$ tartrate. Crystals were cryoprotected in mother liquor containing $25 \%$ glycerol.

Data Collection, Structural Determination, and Refinement. Data were collected at the Diamond Light Source beamline I-04 at a wave length of 0.92819 Å with a PILATUS 6M-F detector. Integration of images was performed using MOSFLM (37) and scaled using Aimless (38). The structure was solved by molecular replacement in Phaser (39) using the apo Lbpro [Protein Data Bank (PDB) ID code 1qol (23)] and ISG15 CTD [PDB ID code 3phx (18)] structures. Model building and refinement were performed using COOT (40) and PHENIX (41). Table S1 shows statistics. Structural figures were created using PyMol (https://pymol. org/2/). The pro-origami server (42) was used to build the topology diagram.

In Vitro Self-Processing Assays. Lb $b^{\text {pro }}$ intramolecular cleavage was monitored by in vitro translation assays (15). To this end, the PCITE-1d plasmid containing the sequence for the Lbpro VP4/VP2 or Lb ${ }^{\text {pro }}$ mutants (L92A, P99A, L102A) was linearized with SalI followed by in vitro transcription (43). Template DNA was digested with DNase, and RNA was purified by phenolchloroform extraction. Then, rabbit reticulocyte lysates were programmed with in vitro-transcribed RNA of the precursor protein $\mathrm{Lb}^{\text {pro }} \mathrm{VP} 4 / \mathrm{VP} 2$ in the presence of [35S] methionine (43), and aliquots were removed at the specified times. The reaction was stopped in stop solution [61\% (vol/vol) $2 \times$ Laemmli sample buffer, $0.6 \mathrm{mM}$ methioninecysteine mix, 37\% ( $\mathrm{vol} / \mathrm{vol}) \mathrm{dH} 2 \mathrm{O}$ ]. Radiolabeled proteins were detected by analysis on a 17.5\% SDS/PAGE gel followed by autoradiography. Gels were incubated twice with $20 \mathrm{~mL}$ enhancing solution containing $1 \mathrm{M}$ sodium salicylate and $45 \%$ methanol for $15 \mathrm{~min}$ before being dried on Whatman paper using an SAVANT slab gel drier and exposed to a CL-X Posure film (Thermo Scientific) for $18 \mathrm{~h}$ at $-80{ }^{\circ} \mathrm{C}$. Subsequently, the film was thawed and developed using an AGFA Curix660. Intermolecular cleavage of endogenous eIF4G was monitored by analyzing $10 \mu \mathrm{L}$ of translation mix on a $6 \%$ SDS/PAGE gel and subsequent Western blotting with anti-eIF4GI antibody (a gift from Robert Rhoads, Louisiana State University HealthSciences Center, Shreveport, LA).

Cell Culture, Transfections, and Incubation with $\mathbf{L b}^{\text {pro }}$. HeLa cells obtained from ATCC were grown to $80 \%$ confluency before transfection with the indicated plasmids as previously described (12). Cells were harvested and lysed in lysis buffer (50 mM Tris,pH 8.0, $4 \mathrm{M}$ urea, $50 \mathrm{mM} \mathrm{NaCl}, 10 \mathrm{mM}$ chloroacetamide, $1 \mathrm{mM}$ EDTA, $1 \mathrm{mM} \mathrm{PMSF}, 0.1 \%$ Nonidet $\mathrm{P}-40,25 \mathrm{mM}$ sucrose, $5 \%$ glycerol). Cells were briefly sonicated to shear DNA and further supplement lysis. Cell debris was cleared by centrifugation at $21,000 \times \mathrm{g}$ for $10 \mathrm{~min}$ at $4{ }^{\circ} \mathrm{C}$. Unreacted chloroacetamide was quenched with $10 \mathrm{mM}$ DTT to prevent alkylation of the $\mathrm{Lb}^{\text {pro }}$ active site cysteine. Total protein was quantified using Bradford methods. Before incubation with $\mathrm{Lb}^{\text {pro }}$, the concentration of urea was diluted to $1 \mathrm{M}$; $100 \mu \mathrm{L}$ of $5 \mathrm{mg} / \mathrm{mL}$ total 
protein was incubated with $2 \mu \mathrm{M} \mathrm{Lb} b^{\text {pro }}$ for 30 min at $37^{\circ} \mathrm{C}$. Reactions were quenched with LDS sample buffer at the indicated time points. Samples were resolved on a 4-12\% Bis-Tris gel (Invitrogen) before being transferred to nitrocellulose membranes by Western blotting. Anti-FLAG (Sigma-Aldrich, M2), anti-ISG15 (Sigma-Aldrich, SAB1408653), anti-ubiquitin (Millipore, 07-375), and anti-GlyGly (Lucerna Inc., GX41) primary antibodies were used in Western blots. All Western blots, unless stated otherwise, were incubated for 16h at a 1:1,000 primary antibody dilution in phosphate buffered saline with Tween 20 (PBS-T) $+5 \%$ BSA. The primary antibody dilution for anti-FLAG Western blots was 1:10,000. After primary antibody incubation, Western blots were incubated at a 1:5,000 secondary antibody dilution overnight inPBS-T+5\% milk prior to being visualized by chemiluminescence. All antibody incubation steps were performed at $4{ }^{\circ} \mathrm{C}$.

Recombinant Mengovirus Infection Assays. HeLa-R19 cells were cultured in DMEM (Lonza) supplemented with $10 \%$ (vol/vol) FCS. Recombinant viruses were derived from the pM16.1-VFETQG-Zn infectious clone as described previously (29). In short, genes of interest were inserted between the XhoI/NotI restriction sites of the PM16.1-VFETQG$\mathrm{Zn}$ infectious clone. Viruses were recovered by transfection of run-off RNA transcripts into BHK-21 cells. On total cytopathic effect, cells were subjected to two freeze-thaw cycles, the cell debris was pelleted at 4,000 $\mathrm{xg}$ for $15 \mathrm{~min}$, and the virus was concentrated by ultracentrifugation through a $30 \%$ sucrose cushion at $140,000 \times \mathrm{g}$ for $16 \mathrm{~h}$ in an SW32Ti rotor. HeLa R19 cells were seeded in $10-\mathrm{cm}$ dishes $\left(2.0 \times 10^{6}\right.$ cells per dish). The next day, cells were infected (multiplicity of infection=10) with the indicated viruses. At 2, 4, 6, or 8 $\mathrm{h}$ postinfection, cells were released using trypsin, washed once in PBS, and lysed in $100 \mu \mathrm{L}$ lysis buffer [100 mM Tris, pH 8.0, 1 mM EDTA, $50 \mathrm{mM} \mathrm{NaCl}, 1 \%$ Nonidet P-40, protease inhibitor mix (Roche)]. Postnuclear lysate was obtained by centrifugation at 15,000xg at 4 ${ }^{\circ} \mathrm{C}$ for $15 \mathrm{~min}$. The amount of total protein in the lysates was determined using BCA assay (ThermoFisher).

FMDV Infection Assays. BHK-21 cells grown to $90 \%$ confluency in 24-well plates were transfected with FLAG-ISG15 and the ISG15 machinery (UBE1L, UBE2L6, and HERC5) and grown overnight. Transfected cells were then infected with FMDVO1 Kaufbeuren diluted in virus growth medium (BHK cell culture medium containing $1 \% \mathrm{FBS}$ ). After $\mathrm{h}$, the FMDV inoculum was removed, the cells were washed once with viral growth medium, and then, they were incubated in virus growth medium for the remainder of the infection. At $\mathrm{ih}$ intervals postinfection (up to $5 \mathrm{~h}$ ), duplicate wells were washed once with PBS, and the cells were lysed in lysis buffer (50 mM Tris, pH 7.5, 0.5\% IGEPAL, $120 \mathrm{mM} \mathrm{NaCl}$ ).The lysates were clarified by centrifugation, and total protein was quantified using BCA assay

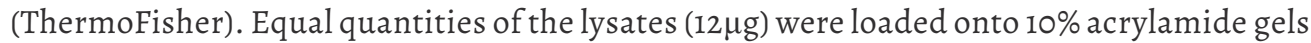
and subjected to SDS-PAGE. Proteins were transferred to nitrocellulose membranes and blotted with either FLAG M2 (Sigma-Aldrich) or GlyGly (Lucerna Inc.) primary antibodies and mouse secondary antibody diluted in blocking buffer (5\% milk and 0.1\%Tween in PBS). Blots were visualized by chemiluminescence. 


\section{Chapter 4}

Dissecting distinct proteolytic activities of FMDV $L^{\text {pro }}$ implicates cleavage and degradation of RLR signaling proteins, not its deISGylase/DUB activity, in type I interferon suppression

Linda J. Visser ${ }^{1}$, Chiara Aloise $^{1, *}$, Kirby N. Swatek ${ }^{2,5, *}$, Gisselle N. Medina $^{3,8, *}$, Karin M. Olek $^{4, *}$, Huib H. Rabouw ${ }^{1,8}$, Raoul J. de Groot ${ }^{1}$, Martijn A. Langereis ${ }^{1,8}$, Teresa de los Santos ${ }^{3}$, David Komander ${ }^{2,6,7}$, Tim Skern ${ }^{4}$, Frank J.M. van Kuppeveld ${ }^{1 *}$

${ }^{1}$ Virology Division, Department of Infectious Diseases and Immunology, Faculty of Veterinary Medicine, Utrecht University, The Netherlands

${ }_{2}^{2}$ Protein and Nucleic Acid Chemistry Division, Medical Research Council Laboratory of Molecular Biology, CB2 OQH Cambridge, United Kingdom

${ }^{3}$ United States Department of Agriculture, Agricultural Research Service, Foreign Animal Disease Research Unit, Plum Island Animal Disease Center, Orient, NY 11957, USA

${ }^{4}$ Department of Medical Biochemistry, Max Perutz Labs, Vienna Biocenter, Medical University of Vienna, A-1030 Vienna, Austria

${ }^{5}$ Department of Molecular Machines and Signaling, Max Planck Institute of Biochemistry, Martinsried, Germany

${ }^{6}$ Ubiquitin Signaling Division, The Walter and Eliza Hall Institute of Medical Research, Parkville, AU

'Department of Medical Biology, The University of Melbourne, Melbourne, AU

${ }^{8}$ Present addresses: GNM is at Kansas State University College of Veterinary Medicine, Manhattan, USA; HHR is at the Hubrecht Institute, Utrecht, The Netherlands; and MAL is at MSD Animal Health, Boxmeer, The Netherlands.

\# These authors contributed equally, " Corresponding author. 


\section{Abstract}

The type I interferon response is an important innate antiviral pathway. Recognition of viral RNA by RIG-I-like receptors (RLRs) activates a signaling cascade that leads to type I interferon $(\mathrm{IFN}-\alpha / \beta)$ gene transcription. Multiple proteins in this signaling pathway (e.g. RIG-I, MDA5, MAVS, TBK1, IRF3) are regulated by (de)ubiquitination events. Most viruses have evolved mechanisms to counter this antiviral response. The leader protease $\left(\mathrm{L}^{\text {pro }}\right)$ of foot-and-mouth-disease virus (FMDV) has been recognized to reduce IFN$\alpha / \beta$ gene transcription; however, the exact mechanism is unknown. The proteolytic activity of $\mathrm{L}^{\text {pro }}$ is vital for releasing itself from the viral polyprotein and for cleaving and degrading specific host cell proteins, such as $\mathrm{eIF}_{4} \mathrm{G}$ and NF- $\mathrm{KB}$. In addition, $\mathrm{L}^{\text {pro }}$ has been demonstrated to have deubiquitination/deISGylation activity. $\mathrm{L}^{\text {pros }}$ deubiquitination/ deISGylation activity and the cleavage/degradation of signaling proteins have both been postulated to be important for reduced IFN- $\alpha / \beta$ gene transcription. Here, we demonstrate that TBK1, the kinase that phosphorylates and activates the transcription factor IRF3, is cleaved by $\mathrm{L}^{\text {pro }}$ in FMDV-infected cells as well as in cells infected with a recombinant EMCV expressing $\mathrm{L}^{\text {pro }}$. In vitro cleavage experiments revealed that $\mathrm{L}^{\text {pro }}$ cleaves $\mathrm{TBK} 1$ at residues 692-694. We also observed cleavage of MAVS in HeLa cells infected with EMCV-L pro, but only observed decreasing levels of MAVS in FMDV-infected porcine LFPK $\alpha V \beta 6$ cells. We set out to dissect $\mathrm{L}^{\text {pro's }}$ ability to cleave RLR signaling proteins from its deubiquitination/ deISGylation activity, to determine their relative contributions to the reduction of IFN- $\alpha / \beta$ gene transcription. The introduction of specific mutations, of which several were based on the recently published structure of $\mathrm{L}^{\text {pro }}$ in complex with ISG15, allowed us to identify specific amino acid substitutions that separate the different proteolytic activities of $\mathrm{L}^{\text {pro }}$. Characterization of the effects of these mutations revealed that $\mathrm{L}^{\text {pro's }}$ ability to cleave RLR signaling proteins but not its deubiquitination/deISGylation activity correlates with the reduced IFN- $\beta$ gene transcription.

\section{Author's summary}

Outbreaks of the picornavirus foot-and-mouth disease virus (FMDV) have significant consequences for animal health and product safety and place a major economic burden on the global livestock industry. Understanding how this notorious animal pathogen suppresses the antiviral type I interferon (IFN- $\alpha / \beta$ ) response may help to develop countermeasures to control FMDV infections. FMDV suppresses the IFN- $\alpha / \beta$ response through the activity of its Leader protein $\left(\mathrm{L}^{\text {pro }}\right)$, a protease that can cleave host cell proteins. $\mathrm{L}^{\text {pro }}$ was also shown to have deubiquitinase and deISGylase activity, raising the possibility that $\mathrm{L}^{\text {pro }}$ suppresses IFN- $\alpha / \beta$ by removing ubiquitin and/or ISG15, two posttranslational modifications that can regulate the activation, interactions and localization of (signaling) proteins. Here, we show that TBKi and MAVS, two signaling proteins that are important for activation of IFN $\alpha / \beta$ gene transcription, are cleaved by $L^{\text {pro }}$. By generating $L^{\text {pro }}$ mutants lacking either of these two activities, we demonstrate that $\mathrm{L}^{\text {pros }} \mathrm{S}$ ability to cleave signaling proteins, but not its deubiquitination/ deISGylase activity, correlates with suppression of IFN- $\beta$ gene transcription.

\section{Introduction}

A virally infected cell activates a plethora of antiviral responses. One of the best-known antiviral responses is the induction of type I interferons (IFN- $\alpha / \beta$ ). Replication of the viral genome generates double-stranded RNA (dsRNA) replication intermediates that can be recognized by cytoplasmic RIG-I like receptors (RLRs). For example, picornaviruses, small $(\sim 30 \mathrm{~nm})$ non-enveloped viruses with a positive-sense RNA genome, synthesize replication intermediates that are predominantly recognized by MDA5 (1-4). Upon recognition of viral dsRNA, MDA5 interacts with MAVS, which subsequently activates TRAF3 and TBK1. TBKI phosphorylates the transcription factors IRF3 and IRF7, resulting in their activation and dimerization. Simultaneously, TRAF3 interacts with the IKK complex to activate the

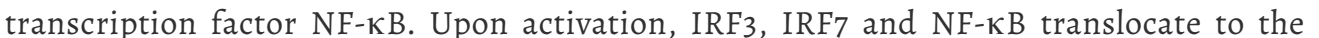
nucleus, where they induce expression of IFN- $\alpha / \beta$ and other proinflammatory cytokines. Subsequent IFN- $\alpha / \beta$ signaling via the type I IFN receptor (IFNAR) and the JAK-STAT pathway induces the expression of hundreds of interferon stimulated genes (ISGs) (reviewed in $(5,6)$ ).

IFN- $\alpha / \beta$ gene transcription is extensively regulated by post-translational modification of RLRs and their downstream signaling proteins, including phosphorylation and ubiquitination. Ubiquitin is a $8.5 \mathrm{kDa}$ protein that can be covalently linked through an $\varepsilon$-amino peptide linkage to lysine residues in target proteins. Within the RLR signaling pathway RIG-I, MAVS, TBK1, TRAF3, TRAF6 and IKK $\gamma$ are ubiquitinated and this affects their molecular interactions, localization, stability, or activity (reviewed in $(7,8)$ ). Ubiquitination of RLR signaling proteins can both positively and negatively regulate the signaling pathway, which allows for rapid fine-tuning of the innate immune response against viral infection (reviewed in (7-9)). Consequently, many viruses encode enzymes with deubiquitinating (DUB) activity to manipulate the RLR signaling pathway and thereby suppress expression of IFN- $\alpha / \beta$ (reviewed in (9)).

In addition to ubiquitin, there are multiple ubiquitin-like modifiers, which can also be attached to target proteins. Of special interest is ISG15, an IFN-induced modifier of 17.5 $\mathrm{kDa}$ comprised of two ubiquitin-like domains in tandem. The exact antiviral properties of ISG15 are not yet fully understood (reviewed in $(10,11)$ ). Early work on ISG15 depended on mouse models and showed that expression of ISG15 protected mice from viral infection (12- 
15). However, important biological and structural differences between ISG15 of murine and human origin have since been reported (16-19). More recently, a picture is emerging that proteins are ISGylated co-translationally, explaining why predominantly viral proteins and ISGs are ISGylated upon infection in humans (20). ISGylation of RLR signaling proteins has been reported, but the effect of these modifications on the outcome of the signaling pathway is still unclear ((21-24), reviewed in (11)). In addition, ISG15 has been reported to act as a cytokine $(25,26)$.

IFN- $\alpha / \beta$ signals in autocrine and paracrine ways to induce a tissue-wide antiviral state, thereby limiting viral spread. To establish infection in their host, it is essential for viruses to suppress both the RLR signaling pathway and the downstream signaling of IFN- $\alpha / \beta$. Affecting protein levels of important signaling molecules, either via cleaving them or inducing their degradation, is a strategy commonly used by viruses to suppress antiviral signaling (27-30). One such example is the picornavirus foot-and-mouth disease virus (FMDV). FMDV is a member of the genus Aphthovirus, which also contains bovine rhinitis $A$ and $B$ viruses, and equine rhinitis A virus (ERAV). The genetic information on the FMDV RNA genome is translated as one polyprotein that is autocatalytically processed into the mature proteins, two of which have been shown to possess proteolytic activity and also been implicated in suppressing IFN- $\alpha / \beta$ induction (reviewed in(31)). The $3 \mathrm{C}^{\mathrm{pro}}$, the protease that processes the majority of cleavage sites on the polyprotein, cleaves NF- $\kappa \mathrm{B}$ essential modulator (NEMO), an adaptor protein that is essential to activate the NFKB and IRF signaling pathways (32).

The second protease on the polyprotein implicated in suppressing IFN- $\alpha / \beta$ induction is $\mathrm{L}^{\text {pro, }}$ a papain-like cysteine protease located at the $\mathrm{N}$-terminus of the polyprotein (33). Once synthesized, $\mathrm{L}^{\text {pro }}$ immediately frees itself from the growing peptide chain by autocleavage at its own C-terminus. $L^{\text {pro }}$ then efficiently cleaves the two isoforms of eIF (eukaryotic initiation factor) $4 \mathrm{G}$ to reduce protein synthesis from cellular mRNA (34) and suppresses the induction of IFN- $\alpha / \beta$ via several mechanisms. $L^{\text {pro }}$ has been shown to induce the degradation of NF- $\mathrm{KB}$ subunit p65/RelA $(35,36)$, and decrease the levels of IFN regulatory factor 3 (IRF3) and IRF7 (37). Further, $\mathrm{L}^{\text {pro }}$ can also interact with ADNP, a negative regulator of transcription (38). In addition to cleaving or degrading important signaling molecules, $\mathrm{L}^{\text {pro }}$ possesses deubiquitinase (DUB) activity which has been proposed to modulate RLR signaling (39). A subsequent study demonstrated that $\mathrm{L}^{\text {pro }}$ should be predominantly regarded as a deISGylase rather than a DUB as biochemical evidence showed that $L^{\text {pro }}$ has a 1000 -fold higher affinity for ISG15 than for ubiquitin (40). Structural studies and biochemical studies have shown separate substrate binding sites on $\mathrm{L}^{\text {pro }}$ for the viral polyprotein, the isoforms of eIF4G as well as for ubiquitin and ISG15 (40-42), suggesting that it may be possible to uncouple the activities of $\mathrm{L}^{\text {pro }}$ by the introduction of specific amino acid substitutions.
We therefore set out to uncouple the different activities of $\mathrm{L}^{\text {pro }}$ to discover whether $\mathrm{L}^{\text {pro }}$ suppresses RLR signaling through its deISGylase/DUB activity or through its ability to cleave and degrade multiple RLR signaling proteins. In this work, utilizing encephalomyocarditis virus (EMCV) expressing FMDV L pro (EMCV-L ${ }^{\text {pro }}$ ), we identify MAVS and TBK 1 as new $L^{\text {pro }}$ substrates and determined the cleavage site in TBK1. By introducing specifically designed mutations into $L^{\text {pro }}$, we further identified residues that are important for either the cleavage/degradation of RLR signaling proteins or for its deISGylase/DUB activity, thereby uncoupling the two catalytic activities of $\mathrm{L}^{\text {pro. }}$. We demonstrate that cleavage/degradation of RLR signaling proteins, but not the deISGylase/DUB activity of $\mathrm{L}^{\text {pro, }}$, correlates with suppressing IFN- $\alpha / \beta$ gene transcription.

\section{Results}

FMDV L $L^{\text {pro }}$ reduces IFN- $\beta$ mRNA induction when introduced into a recombinant EMCV containing an inactive stress antagonist. To study the effects of $\mathrm{L}^{\text {pro }}$ on the induction of type I IFN in picornavirus-infected cells, we used two previously generated recombinant viruses; EMCV- $\mathrm{L}^{\mathrm{Zn}}$, which contains inactivating mutations in the zinc-finger domain of the Leader (i.e. EMCV's RLR signaling antagonist) $(1,43,44)$, and EMCV-L $\mathrm{L}^{\text {pro }}$, which was derived from EMCV- $\mathrm{L}^{\mathrm{Zn}}$ and additionally encodes FMDV $\mathrm{L}^{\text {pro }}$ at the $\mathrm{N}$-terminus of its polyprotein (Fig IA) (45). We also constructed a similar recombinant EMCV carrying a catalytically inactive $\mathrm{L}^{\text {pro }}$ (i.e. EMCV-L $\mathrm{L}^{\text {pro }} \mathrm{C} 5 \mathrm{IA}$ ) (46). To determine whether $\mathrm{EMCV}-\mathrm{L}^{\text {pro }}$ can suppress IFN- $\beta$ induction, we infected HeLa cells with EMCV, EMCV-L $\mathrm{L}^{\mathrm{Zn}}, \mathrm{EMCV}-\mathrm{L}^{\text {pro }}$ or EMCV-L $\mathrm{L}^{\text {pro }} \mathrm{C} 51 \mathrm{~A}$ and determined the IFN- $\beta$ mRNA levels over time via RT-qPCR analysis (Fig 1B). Consistent with previous studies $(37,39,47)$, wt $\mathrm{L}^{\text {pro }}$, but not $\mathrm{L}^{\text {pro }} \mathrm{C} 51 \mathrm{~A}$, reduced the induction of IFN- $\beta$ mRNA approximately 10 -fold, indicating that the catalytic activity of $\mathrm{L}^{\text {pro }}$ is needed to suppress RLR signaling. In conclusion, the viruses that we generated

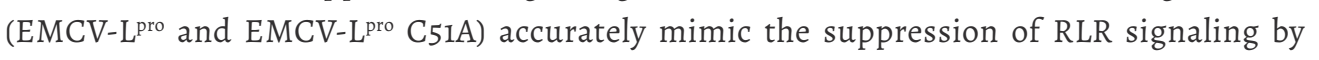
$\mathrm{L}^{\text {pro }}$ as previously reported for FMDV-infected cells (47), providing us a model system to determine the mechanism via which $\mathrm{L}^{\text {pro }}$ suppresses type I IFN induction.

$\mathrm{L}^{\text {pro }}$ cleaves the RLR signaling proteins MAVS and TBK1. $L^{\text {pro }}$ has been reported to degrade important signaling proteins such as the p65 subunit of NF- $\mathrm{KB}, \operatorname{IRF} 3$ and $\operatorname{IRF} 7(35,37)$. To determine whether $L^{\text {pro }}$ targets additional RLR signaling proteins, we subjected cell lysates of HeLa cells infected with EMCV-L $\mathrm{L}^{\mathrm{Zn}}$, EMCV-L pro or EMCV-L $\mathrm{L}^{\text {pro }} \mathrm{C} 51 \mathrm{~A}$ to Western Blot analysis for the signaling proteins MAVS, TBK1 and IRF3, as well as the known $\mathrm{L}^{\text {pro- }}$ substrates eIF4G and G3BPI (Fig 2A). EMCV capsid proteins and tubulin served as infection and loading controls, respectively. Infection with EMCV-L $\mathrm{L}^{\text {pro, }}$, but not EMCV-L $\mathrm{L}^{\text {pro }} \mathrm{C} 51 \mathrm{~A}$, resulted in the rapid cleavage of eIF4G (from 4 hpi onwards) and the cleavage of $\mathrm{G}_{3} \mathrm{BP}$ 
(from 6 hpi onwards). We did not observe cleavage or degradation of IRF3 as was suggested by others (37). In addition to these known cleavages, we observed cleavage of MAVS and TBKi at 8 hpi. For MAVS, we observed multiple cleavage products ranging in apparent molecular weight from $\sim 45 \mathrm{kDa}$ to $\sim 35 \mathrm{kDa}$. TBKi cleavage resulted in a single cleavage product with an apparent molecular weight of $\sim 90-95 \mathrm{kDa}$. We also attempted to detect MDA5 and investigate whether this dsRNA sensor is targeted by $\mathrm{L}^{\text {pro. }}$. Unfortunately, the low levels of MDA5 prevented us from detecting the endogenous protein. MDA5 expression could be boosted by pretreatment with recombinant IFN- $\alpha 2$, but IFN- $\alpha 2$ pretreatment inhibited efficient EMCV infection, thereby interfering with the subsequent analysis.

A
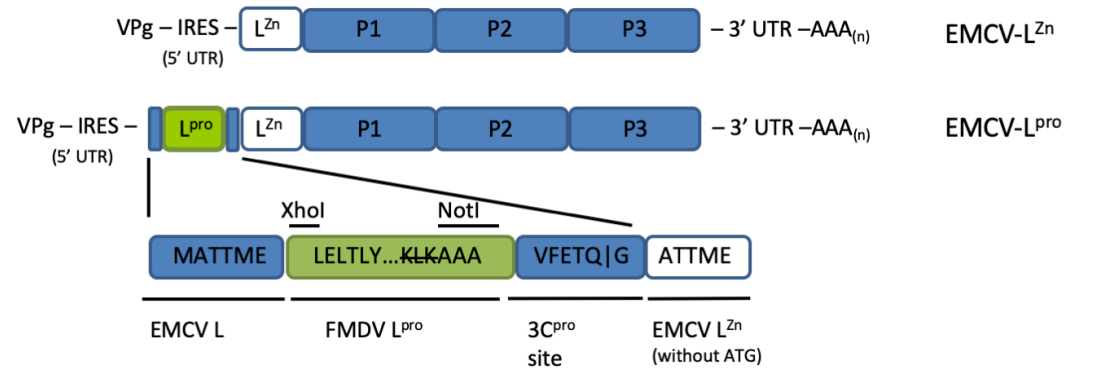

B

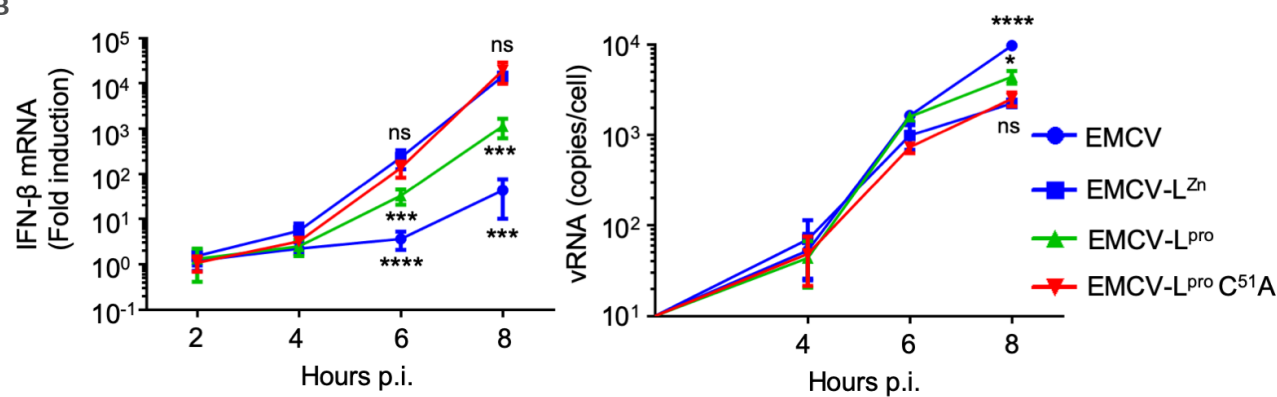

Figure 1: $\mathrm{L}^{\text {pro }}$ reduces IFN- $\beta$ gene transcription when introduced into a recombinant EMCV containing an inactive stress antagonist. (A) Schematic representation of the recombinant EMCV-Ln and EMCV-Lproviruses (the latter was described in detail in (45)). In EMCV-L $\mathrm{L}^{2 n}$, L contains inactivating mutations in its Zn-finger domain (C19A/C22A) which abolishes its ability to suppress antiviral responses. To generate EMCV-L $L^{\text {pro }}$, the L $b^{\text {pro }}$ gene of FMDV O-strain was introduced at the $5^{\prime}$ end of the EMCV-L $\mathrm{L}^{\mathrm{Zn}}$ open reading frame. The L $^{\text {pro }}$ cleavage site at its own C-terminus was mutated to AAA. Instead $\mathrm{L}^{\text {pro }}$ is released from this viral polyprotein via an $\mathrm{EMCV}{ }_{3} \mathrm{C}$ cleavage site. (B) Hela R19 cells were infected at $\mathrm{MOI} 10$ with the indicated viruses and cells were lysed at 2,4,6,8 h pi. Total RNA was isolated and used for RT-qPCR analysis for IFN- $\beta$ and actin $m R N A$, and EMCV VRNA. The IFN- $\beta$ levels are depicted as a fold induction compared to levels in mock-infected cells, after correction for actin mRNA levels. The EMCV VRNA is depicted as a copy number per cell, calculated from a plasmid standard. Error bars depict the SD. One-way ANOVA with the Dunnet post hoc test was used to determine

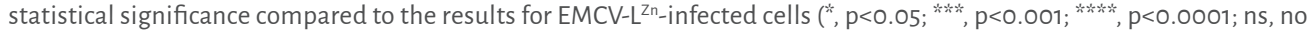
significant difference).
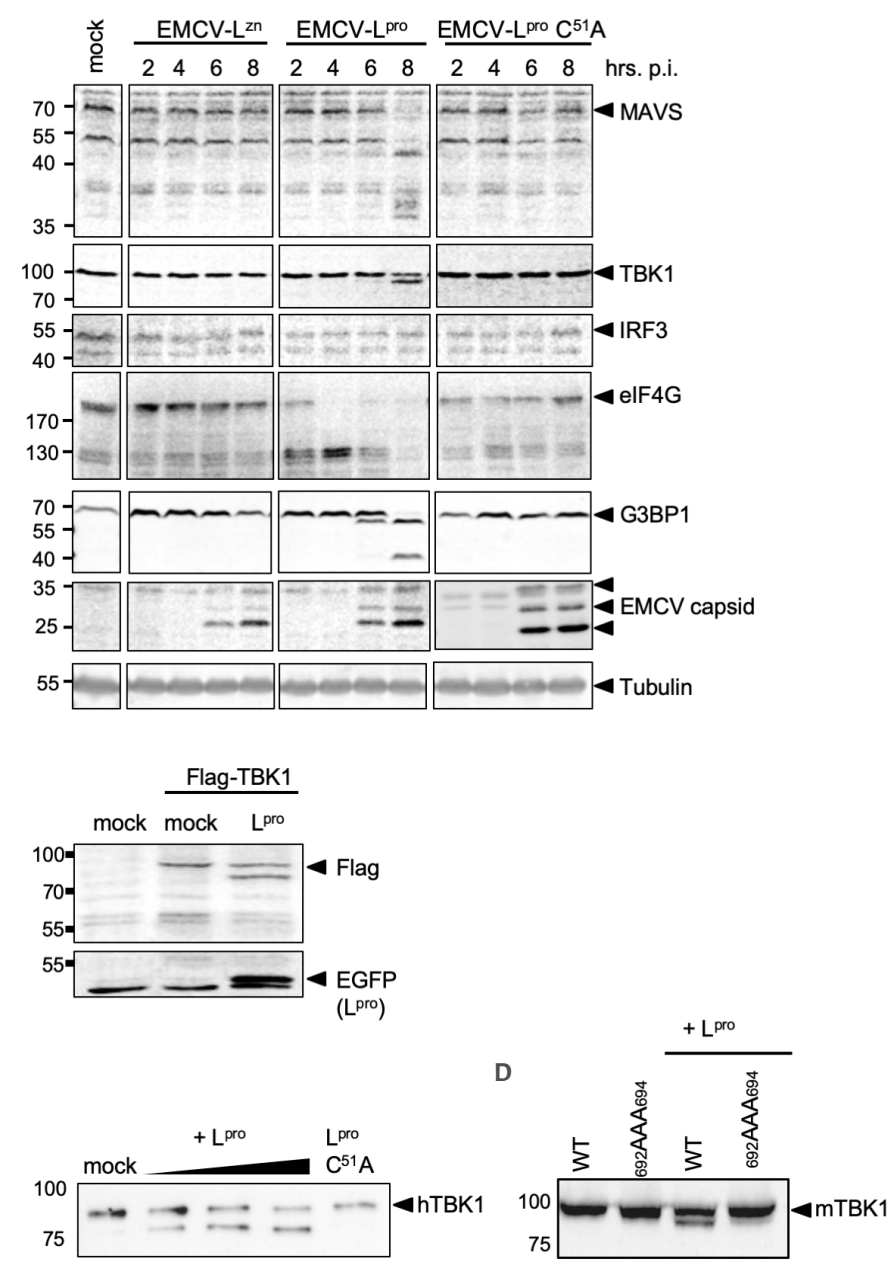

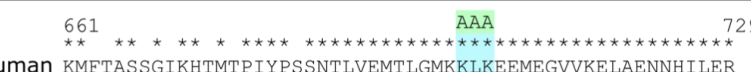

$$
\begin{aligned}
& \text { Mouse KMLAASGGVKHAMAPI YPSSNTL VEMTLGMKKLKEEMEGVVKELLENNAHILER } \\
& \text { Pig KMFAASSGIRHTMTP YYPCSNTLVEMTLGMRKLKEEMEGVVKELAENNHILER }
\end{aligned}
$$

Figure 2: L pro cleaves MAVS and TBK1. (A) HeLa R19 cells were infected at MOI 10 with the indicated viruses and lysed at $2,4,6,8 \mathrm{~h}$ pi. Cell lysates were subjected to Western Blot analysis for MAVS, TBK1, IRF3, elF4GI, C3BP1, EMCV capsid proteins and tubulin. (B) HEK293T cells were transfected with $1 \mu \mathrm{g}$ pcDNA-FLAG-TBK1 plasmid and $0.5 \mu \mathrm{g}$ plRES-CFPLpro plasmid. $16 \mathrm{~h}$ post transfection cells were lysed and lysates were subjected to Western Blot analysis for FLAC and CFP. (C) His-hTBK1 was incubated with o- $3 \mu \mathrm{g} \mathrm{sL}$ pro or $3 \mu \mathrm{g} \mathrm{sL} L^{\text {rro }} \mathrm{C} 51 \mathrm{~A}$ for $2 \mathrm{~h}$ and reaction mixtures were subsequently subjected to Western Blot analysis for His. (D) HeLa OHIO cells were transfected with 7 Hg pCS2-6Myc-mTBK1 or pCS2-

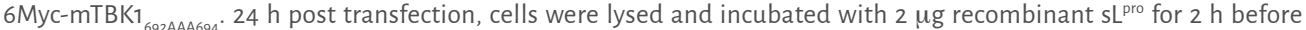
being subjected to Western Blot analysis for HA. (E) Comparison of TBK1 amino acids 661-729 of human and mouse origin. Asterisks denote conserved residues. KLK at position 692-694 was mutated to AAA. 
We next focused our attention to identifying the cleavage site in TBK1. To this end, we overexpressed N-terminally FLAG-tagged TBKi together with GFP-tagged L $^{\text {pro }}$ and performed Western Blot analysis. As seen in Fig 2B, GFP-L $\mathrm{L}^{\text {pro }}$ was able to cleave Flag-TBK1. We observed an $\alpha$ FLAG-reactive cleavage product migrating at $\sim 90-95 \mathrm{kDa}$, the same apparent molecular weight as the cleavage product we observed in EMCV-L $\mathrm{L}^{\text {pro }}$ infected cells (Fig 2A), suggesting that $\mathrm{L}^{\text {pro }}$ cleaves TBKi at its C-terminus. We also co-incubated recombinant His-TBK1 with increasing amounts of recombinantly expressed $L^{\text {pro }}$ and $L^{\text {pro }}$ C51A (Fig 2C). The in vitro incubation of His-TBK1 with wt $\mathrm{L}^{\text {pro }}$ also resulted in a $\sim 90-95 \mathrm{kDa}$ $\alpha$ His-reactive cleavage product, confirming that $\mathrm{L}^{\text {pro }}$ cleaves TBKI at its $\mathrm{C}$-terminus and does not rely on other cellular factors. Incubation of His-TBKi with catalytically inactive $L^{\text {pro }}$ did not result in the formation of a cleavage product, confirming that the cleavage is dependent on $\mathrm{L}^{\text {pro's }}$ proteolytic activity. Subsequently, we showed that $\mathrm{L}^{\text {pro }}$ also cleaves $\mathrm{TBKI}$ of murine origin (Fig 2D), which suggests that $\mathrm{L}^{\text {pro }}$ cleaves TBK1 in a conserved region. We identified residues ${ }_{692} \mathrm{KLK}_{694}$ - which localize at the very C-terminus of TBK1 and are well conserved between human, murine and porcine TBK1 - as a possible cleavage site (Fig $2 \mathrm{E}$ ). Indeed, mutation of these residues prevented the cleavage of TBK1 by L $\mathrm{p}^{\text {pro }}$ (Fig 2D), confirming these residues are the cleavage site.

Upon identifying the cleavage site in TBKi, we next investigated whether the cleavage of TBKI by L ${ }^{\text {pro }}$ inhibits its function in the RLR signaling pathway. To this end, we generated cells in which the endogenous TBKi gene is replaced with a TBKi truncation mutant representative of the $\sim 90-95 \mathrm{kDa}$ cleavage product. We first generated HeLa TBKi k.o. cells using CRISPR/cas9 technology and characterized the remaining RLR signaling capacity of these cells (Fig SiA+B). We found that depletion of TBKI is not sufficient to fully impair RLR signaling, probably because of functional redundancy with IKKe (Fig S1B). TBKi k.o. cells have a $\sim 10$-fold lower IFN- $\beta$ induction upon infection with EMCV-L ${ }^{\mathrm{Zn}}$, transfection of vRNA or upon overexpression of MAVS. As expected, IFN- $\beta$ induction resulting from transfection of IRF3, which acts downstream of TBK1, was not affected in the TBK1 k.o. cells. Subsequently, we expressed full length TBKi or TBKi $\triangle 35 \mathrm{aa}$, which represents the N-terminal cleavage product, in TBKi k.o. cells (Fig SiC) and determined whether expression of TBKi and TBKi $\triangle 35$ aa restored IFN- $\beta$ mRNA expression upon transfection of poly (I:C). Expression of full length TBKı in TBKı k.o. cells fully restored IFN- $\beta$ mRNA induction (Fig S1D) and TBKi $\triangle 35$ aa was similarly efficient in this (Fig S1E), indicating that the $\mathrm{L}^{\mathrm{pro}}$-generated $\mathrm{N}$-terminal cleavage product is signaling competent.
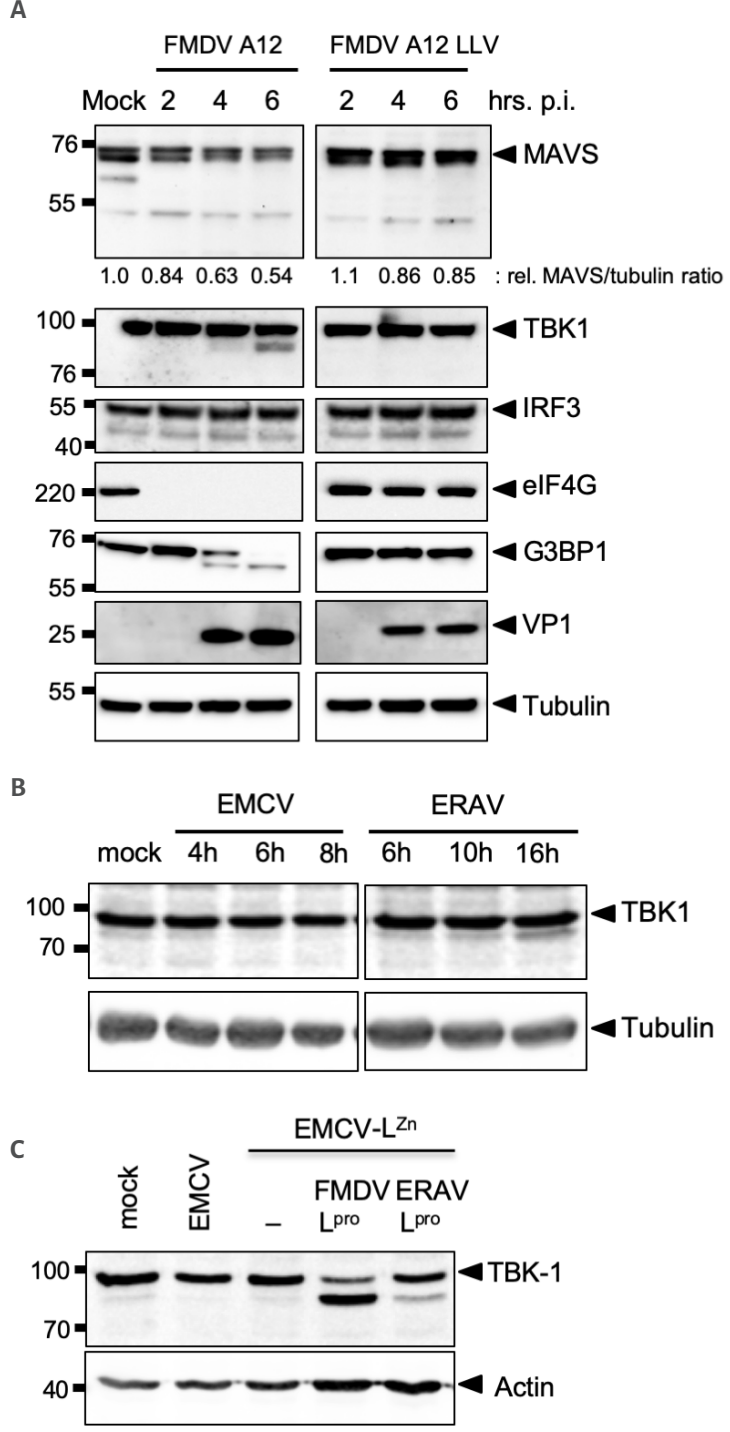

D

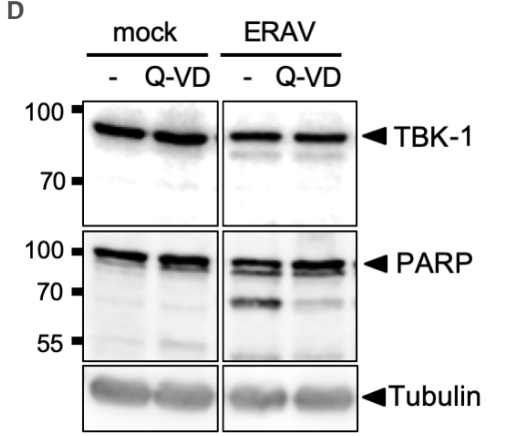

Figure 3: TBK1 is cleaved in aphthovirus infected cells. (A) LFPK $\alpha v \beta 6$ cells were infected with FMDV-A12 or leaderless FMDV-A12 (LLV) at MOI 10 and lysed at the indicated times postinfection. Lysates were subjected to Western blot analysis for MAVS, TBK1, IRF3, elF4C, C3BP1, viral protein $\mathrm{VP}_{1}$ and tubulin. (B) HeLa R19 cells were infected with EMCV or ERAV at MOI 10 and lysed at indicated times post infection. Cell lysates were subjected infection. Cell Iysates were subjected to (C) HeLa R19 cells were infected at MOI 10 with the indicated viruses and lysed at 8 hpi. Cell lysates were subjected to Western Blot analysis for TBK1 and actin. (D) HeLa R19 cells were infected with ERAV at MOI 10. Subsequently, the cells were incubated for $16 \mathrm{~h}$ in the presence or absence of $10 \mathrm{MM}$ $\mathrm{Q}-\mathrm{VD}$, a pan-caspase inhibitor. Cell lysates were subjected to Western Blot analysis for TBK1, PARP and tubulin. 
TBK1 is cleaved during aphthovirus infection. To investigate whether TBK1, MAVS and IRF3 are cleaved during FMDV infection, we infected porcine LFPK $\alpha v b 6$ cells with wt

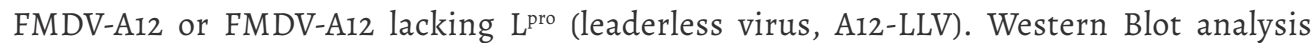
revealed cleavage of TBK1, but not of MAVS or IRF3, during wt FMDV-A12 infection (Fig 3A). TBKi cleavage was observed from 4 hpi onwards upon infection with wt FMDV, but not upon infection with leaderless FMDV. The cleavage product had an apparent molecular weight of $\sim 90-95 \mathrm{kDa}$, consistent with our previous observations of the size of this cleavage product. Although a MAVS cleavage product was not detected during FMDV infection, densitometry analysis revealed a strong and progressive decrease in the relative ratio of MAVS/tubulin from 2-6 hpi with wt FMDV compared to mock-infected cells, whereas only a small decrease was detected in leaderless-infected cells (MAVS/tubulin ratio is indicated in Fig $3 \mathrm{~A}$ ). This suggests that expression of $\mathrm{L}^{\text {pro }}$ induces degradation of MAVS, also in

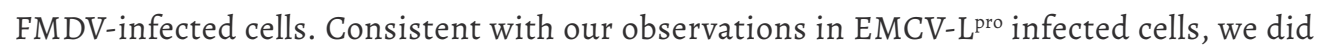
not observe a decrease in IRF3 signal in FMDV-infected cells.

To investigate whether the cleavage of TBKI is conserved amongst aphthoviruses, we infected cells with ERAV, the closest relative of FMDV. Infection with ERAV, but not EMCV, resulted in the cleavage of $\mathrm{TBK}_{1}$ ( $\mathrm{Fig} 3 \mathrm{~B}$ ). However, the cleavage product was less prominent than for EMCV-L $\mathrm{L}^{\text {pro, }}$, suggesting that cleavage was inefficient or infection was delayed. Notably, in our HeLa cells, ERAV displayed a replicative cycle of $\sim 16 \mathrm{~h}$. This is considerably slower than FMDV, which replicates in 6-8 hours. To study TBKi cleavage by the two different $\mathrm{L}^{\text {pro's }}$ irrespective of variation in viral replication kinetics, we infected cells with

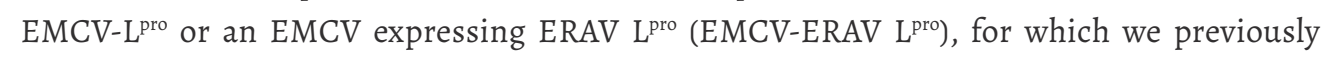
determined the replication kinetics to be similar (45). Both viral proteases cleaved TBKI

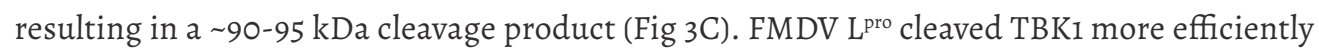
than ERAV $L^{\text {pro }}$, consistent with the results observed during infection with FMDV or ERAV (Fig $3 \mathrm{~A}$ and $3 \mathrm{~B}$ ). Notwithstanding the differences between ERAV L ${ }^{\text {pro }}$ and FMDV L ${ }^{\text {pro }}$, our data demonstrate that the ability to cleave $\mathrm{TBK}_{1}$ is conserved amongst these two aphthoviruses.

We also investigated the effect of the pan-caspase inhibitor Q-VD-PH (Q-VD) on TBK1 cleavage in ERAV-infected cells (Fig 3D). While addition of Q-VD decreased the cleavage of known caspase substrate PARP, the cleavage of TBK1 was unaffected. Collectively, these results demonstrate that $\mathrm{L}^{\text {pro }}$ directly cleaves $\mathrm{TBK} 1$ and that this activity is conserved amongst aphthoviruses.

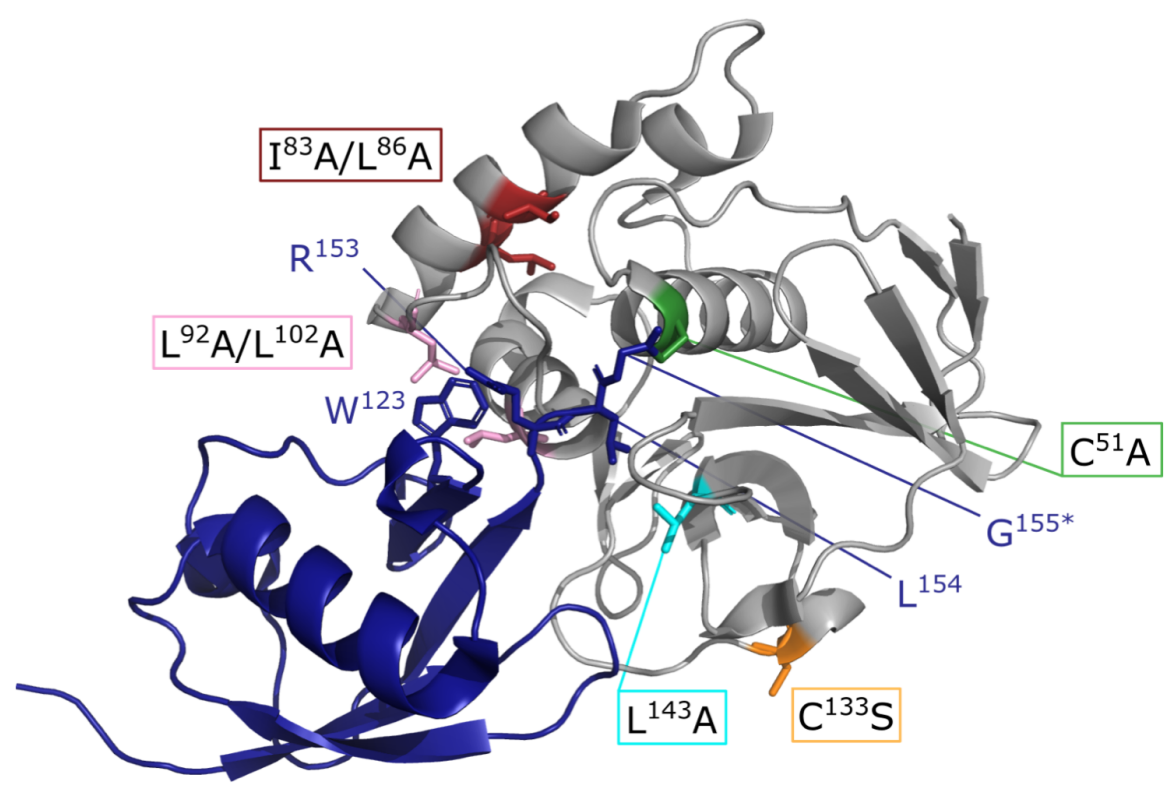

\begin{tabular}{|l|l|l|l|}
\hline Mutation & Affected domain & Phenotype & References \\
\hline $\mathrm{C}^{51} \mathrm{~A}$ & Catalytic site & $\begin{array}{l}\text { Inactivation of catalytic activity } \\
\text { (in vitro assays and ectopic overexpression) }\end{array}$ & 46 \\
\hline $\mathrm{I}^{83 \mathrm{~A} / \mathrm{L}^{86} \mathrm{~A}}$ & $\mathrm{SAP}$ domain & $\begin{array}{l}\text { Reduced DUB activity (ectopic overexpression) } \\
\text { Failure to suppress IFN induction (in infected cells) }\end{array}$ & $36,37,39$ \\
\hline $\mathrm{L}^{\mathrm{L}^{2} \mathrm{~A}}$ & ISG15 binding domain & $\begin{array}{l}\text { Reduced affinity for ISG15 and ubiquitin } \\
\text { (in vitro assays) }\end{array}$ & 40 \\
\hline $\mathrm{L}^{102} \mathrm{~A}$ & ISG15 binding domain & $\begin{array}{l}\text { Reduced affinity for ISG15 and ubiquitin } \\
\text { (in vitro assays) }\end{array}$ & 40 \\
\hline $\mathrm{C}^{133 \mathrm{~S}}$ & elf4G binding domain & $\begin{array}{l}\text { Reduced affinity for elf4G } \\
\text { (in vitro assays) }\end{array}$ & 48 \\
\hline $\mathrm{L}^{143} \mathrm{~A}$ & $\begin{array}{l}\text { Substrate binding } \\
\text { cleft }\end{array}$ & $\begin{array}{l}\text { Broader specificity in binding pocket } \\
\text { (in vitro assays) }\end{array}$ & 49 \\
\hline
\end{tabular}

Figure 4: Visualization and summary of the Lro mutants used in this study. (A) Standard cartoon view of L pro (grey) bound to ISG15 (blue) (PDB: 6FFA). L pro L92 and L102 interact with ISG15 W123, this positions ISC15's C-terminus (R153, L154 and $\mathrm{G}_{155}$ ) in the substrate binding cleft of $\mathrm{L}^{\text {pro }}$. "In this structure a C-terminal propargyl warhead replaced G155 (40). Residues which upon mutation were reported to affect Lpros's structure or function are shown as colored sticks. Green: C51A inactivates Lro's catalytic activity; red: 183A or L86A reduce the DUB activity and IFN induction; pink: L92A and L102A reduce affinity for ISG15; orange: $\mathrm{C}_{133 S} \mathrm{~S}$ reduces affinity for elF4C; aquamarine: L143A predicted to open substrate binding pocket. Drawings were generated using PyMOL. (B) Overview of the reported effects of the introduced mutations in $\mathrm{L}^{\mathrm{pro}}$ on the various proteolytic activities of $\mathrm{L}^{\text {pro }}$, this includes references and the category of the underlying experimental evidence. 
Construction of $\mathrm{L}^{\text {pro }}$ mutants to uncouple cleavage/degradation of RLR signaling proteins from its deISGylase/DUB activity. $L^{\text {pro }}$ also possesses deISGylase and - to a lesser extent - DUB activity $(39,40,42)$, and this latter activity was previously suggested to be important for $L^{\text {pro's }}$ ability to suppress RLR signaling (39). To investigate how $L^{\text {pro }}$ reduces the induction of IFN- $\beta$ gene transcription, we set out to uncouple these two abilities of $L^{\text {pro. }}$. To this end, we introduced previously described mutations in the chimeric EMCV$L^{\text {pro }}$ and determined whether these mutations affect the deISGylase/DUB activity of $L^{\text {pro }}$ and/or its ability to cleave/degrade RLR signaling proteins. A mutation in the SAP domain (I83A/L86A), was previously reported to abolish the ability of $\mathrm{L}^{\text {pro }}$ to suppress type I IFN expression, to degrade signaling proteins (i.e. NF- $\mathrm{KB}$ p65, IRF3 and IRF7), and to disrupt its DUB activity $(36,37,39)$, and was therefore included in our screening. Analysis of the crystal structure of L pro bound to ISG15 suggested that L $^{\text {pro }}$ residues L92, P99 and L102 are important for ISG15 binding (40). Fig 4A shows the structure of $\mathrm{L}^{\text {pro }}$ (grey) in complex with ISG15 (blue) and indicates the residues of ISG15 (W123 and R153, L154, G155) that interact with L $\mathrm{L}^{\text {ro }}$. Mutation of L92, P99 or L102 in L ${ }^{\text {pro }}$ reduced its affinity for ISG15 and impaired its deISGylase activity, without affecting eIF4G cleavage (40). Homology modeling showed that ISG15 and ubiquitin interact with the same surfaces of $\mathrm{L}^{\text {pro }}(40)$, suggesting that $\mathrm{L}^{\text {pro' }} \mathrm{s}$ deISGylase activity also reflects its DUB activity. Two of these mutations (L92A and L102A)

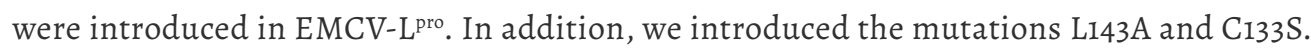
Mutation $\mathrm{C} 133 \mathrm{~S}$ was reported to reduce the affinity of $\mathrm{L}^{\text {pro }}$ for eIF4G (48) whereas mutation of L143 to alanine with its shorter side-chain rescued polyprotein processing in the context of the additional mutation L200F (49). Based on the structure of $\mathrm{L}^{\text {pro }}$, mutation Li43A has been predicted to open up the catalytic pocket. Figure 4 shows the locations of the residues that are mutated in this study (Fig 4A) and summarizes the reported effects of these mutations on $\mathrm{L}^{\text {pro's }}$ various proteolytic activities (Fig 4B).

Effect of $\mathrm{L}^{\mathrm{pro}}$ mutations on cleavage and/or degradation of RLR signaling proteins. First, we determined the effect of the introduced mutations on $L^{\text {pro's }}$ ability to cleave or degrade RLR signaling proteins. We infected Hela cells with EMCV, EMCV-L $\mathrm{L}^{\mathrm{Zn}}$ or the different EMCV-L $\mathrm{L}^{\text {pro }}$ carrying the described mutations, lysed the cells at the indicated timepoints and subjected the lysates to Western Blot analysis for MAVS, TBK1, NF- $\mathrm{KB}$ subunit p65, IRF3, eIF4G and G3BP1, as well as $L^{\text {pro }}$ and EMCV capsid proteins (Fig $5 \mathrm{~A}$ ). Our data show that infection with the different $L^{\text {pro }}$ mutant viruses resulted in $L^{\text {pro }}$ expression and accumulation of EMCV capsid proteins from $6 \mathrm{hpi}$ onwards, which is indicative of efficient infection. Interestingly, the introduced mutations in $\mathrm{L}^{\text {pro }}$ had different effects on the cleavage or degradation of RLR signaling proteins. Upon infection with EMCV-L pro $\mathrm{C} 133 \mathrm{~S}$, we observed a $\sim 2 \mathrm{hr}$ delay in eIF4G cleavage, consistent with a previous report (48). This mutation did not affect the cleavage/degradation of the various RLR signaling proteins. Mutation of L92 or L102 has been reported to reduce the activity of $\mathrm{L}^{\text {pro }}$ towards ISG15, without affecting eIF4G cleavage
(40). Indeed, infection with EMCV-L $\mathrm{L}^{\text {pro }} \mathrm{L} 92 \mathrm{~A}$, resulted in cleavage of eIF4G, as well as all other $\mathrm{L}^{\text {pro }}$ substrates (i.e. MAVS, TBK1, NF- $\mathrm{KB}$ p65 and G3BP1) (Fig 5A). Infection with both EMCV-L $\mathrm{L}^{\text {pro }}$ L92A and EMCV-L $\mathrm{L}^{\text {pro }}$ L102A resulted in efficient cleavage of RLR signaling proteins, confirming that these two $\mathrm{L}^{\text {pro }}$ mutants have the same proteolytic profiles (Fig $5 \mathrm{~B})$. Of note, the cleavage of eIF4G appears to occur faster by $\mathrm{L}^{\text {pro }} \mathrm{L} 92 \mathrm{~A}$ and L102A compared to $\mathrm{wt} \mathrm{L}^{\mathrm{pro}}$, although a more in-depth analysis is necessary to confirm a true difference in eIF4G cleavage kinetics. Importantly, both mutations allow us to separate the deISGylase/ DUB activity of $\mathrm{L}^{\text {pro }}$ from its ability to cleave RLR signaling proteins. Serendipitously, we observed that $\mathrm{L}^{\text {pro }}$ carrying mutation L143A was strongly impaired in degrading NF- $\mathrm{\kappa B}$ p 65 and cleaving MAVS and TBK1, while cleavage of G3BP1 and eIF4G was delayed but could be observed clearly at later timepoints (eIF4G cleavage is $\sim 2 \mathrm{hr}$ delayed, comparable to the delay observed for $\mathrm{L}^{\text {pro }} \mathrm{C} 133 \mathrm{~S}$ ). Mutation of $\mathrm{L}^{\text {pro's }} \mathrm{SAP}$ domain (I83A/L86A), which was previously shown to abolish degradation of NF- $\mathrm{KB}$ p 65 and DUB activity as well as to impair $L^{\text {pros }}$ s ability to reduce IFN- $\beta$ mRNA expression $(36,39)$, also affected $L^{\text {pros }}$ ability to cleave MAVS and TBK1. Overall, our data demonstrate that the mutations have differential effects on the cleavage/degradation of RLR signaling proteins. Importantly, we demonstrate that $\mathrm{L}^{\text {pro }}$ residues L92 and L102, which are essential for its deISGylase/DUB activity, are not essential for is ability to cleave/degrade RLR signaling proteins, indicating that the two different catalytic activities of $\mathrm{L}^{\text {pro }}$ can be uncoupled.

Effect of $\mathrm{L}^{\text {pro }}$ mutations on DUB and deISGylase activities. It has been previously reported that mutations L92A, L102A and I83A/L96A affect L ${ }^{\text {pro's }}$ deISGylase/DUB activity $(39,40)$. To determine the effect of mutations L143A or C133S on these activities, mutant $\mathrm{L}^{\text {pros }} \mathrm{s}$ were expressed and purified from E. coli and in vitro catalytic activities towards ubiquitinTAMRA and ISG15-TAMRA were measured (Fig 6A). L pro $^{\mathrm{C}} 133 \mathrm{~S}$ and L143A displayed wt-like activity towards ISG15 and ubiquitin. We next determined DUB activity of $\mathrm{wt}^{\mathrm{p}} \mathrm{p}^{\text {pro }}, \mathrm{L}^{\text {pro }} \mathrm{C} 51 \mathrm{~A}$, C133S and L143A in cells. To this end, we transfected HEK-293T cells with a combination of HA-ubiquitin, FLAG-RIG-I and increasing amounts of GFP-L $L^{\text {pro }}$ encoding plasmids ( $0.1,0.3$ and $0.5 \mu \mathrm{g}$ ), and visualized HA-tagged ubiquitinated proteins by Western Blot analysis (Fig 6B). FLAG-RIG-I, which was included to monitor the effects of $\mathrm{L}^{\text {pro-induced translational }}$ shut-off on the overexpressed proteins, was clearly detectable even at the highest level of $L^{\text {pro }}$ (i.e. transfection of $0.5 \mu \mathrm{g}$ of $\mathrm{L}^{\text {pro }}$ plasmid), indicating that $\mathrm{L}^{\text {pro-induced translational }}$ shut-off did not significantly reduce the protein expression from the transfected plasmids. Both wt $\mathrm{L}^{\text {pro }}$ as well as the $\mathrm{C} 133 \mathrm{~S}$ and L143A mutants displayed DUB activity upon overexpression in cells, as indicated by the reduction of HA-tagged ubiquitinated proteins upon increasing $\mathrm{L}^{\text {pro }}$ levels. Notably, we observed ubiquitinated proteins upon transfection of low amount of $\mathrm{L}^{\text {pro }} \mathrm{C} 133 \mathrm{~S}$ plasmid ( $0.1 \mu \mathrm{g}$ ), although our in vitro data (Fig 6A) indicated that mutation $\mathrm{C} 133 \mathrm{~S}$ does not reduce the activity for ubiquitin. As the in vitro data are much more quantitative in nature, we consider the relatively decreased DUB activity of $\mathrm{L}^{\text {pro }} \mathrm{C} 133 \mathrm{~S}$ 

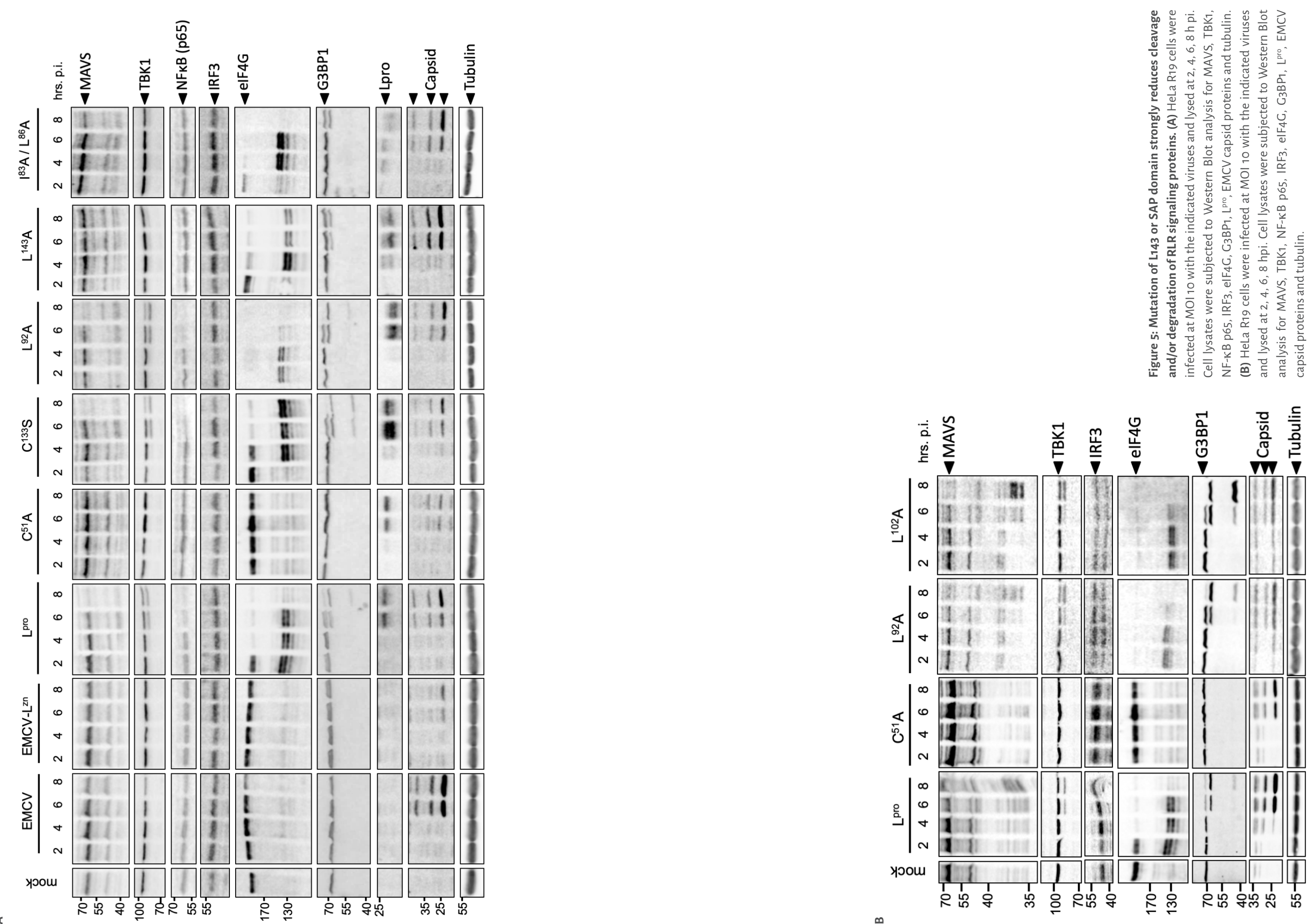
in comparison to $\mathrm{L}^{\text {pro }} \mathrm{Wt}$ or mutant Li43A as observed in Fig 6B the result of variations in expression of the transfected plasmids.
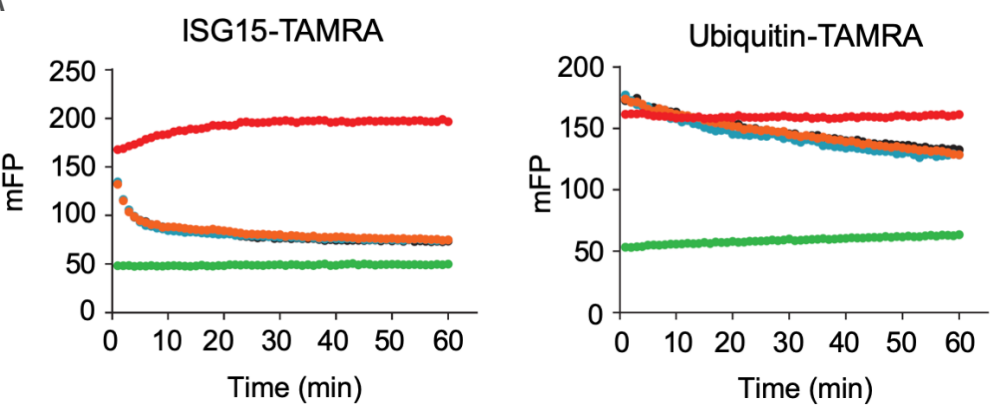

- Pos. control

- Neg. control

- WT L L pro

- $L^{\text {pro }} \mathrm{C}^{133} \mathrm{~S}$

Time (min)

Time (min)

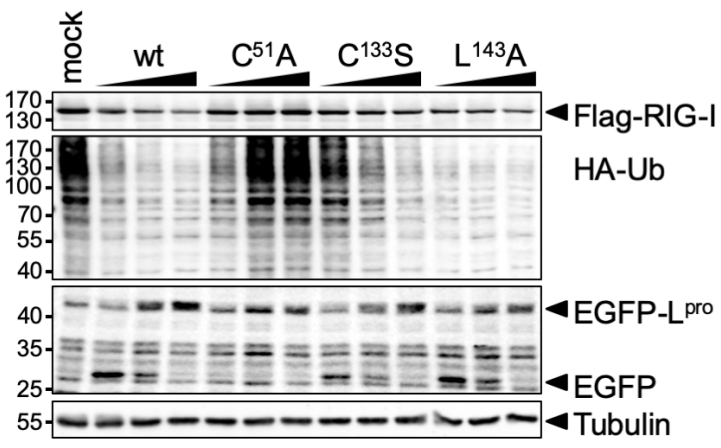

Figure 6: Mutation of $\mathrm{L}_{143}$ or $\mathrm{C}_{133} \mathrm{~S}$ does not affect DUB or delSCylase activities. (A) Ubiquitin-TAMRA or ISG15TAMRA (substrates) were incubated with $10 \mu \mathrm{M}$ or $100 \mathrm{nM} \mathrm{L}^{\text {pro }}$ (wt or indicated mutant), respectively. The change in fluorescent polarization (MFP) was determined over $60 \mathrm{~min}$, with readings recorded every $60 \mathrm{sec}$. The average trace of assays performed in technical triplicate is shown. Substrate only and KG-TAMRA peptide served as respective negative and positive controls. (B) HEK293T cells were transfected with $0.5 \mu \mathrm{g} \mathrm{pcDNA-FLAG-RIC-1,} 0.5 \mu \mathrm{g}$ pCDNA-HA-ubiquitin and $0.1-0.5 \mu \mathrm{g}$ pIRES-CFP-Lpro. $16 \mathrm{~h}$ post transfection, cells were lysed and lysates subjected to Western Blot analysis for FLAC, HA, GFP and tubulin.

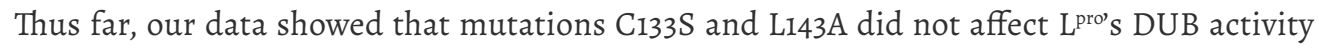
towards a mono-ubiquitin fused to a fluorescent TAMRA molecule. To exclude the possibility that mutations $\mathrm{C} 133 \mathrm{~S}$ and L143A affect $\mathrm{L}^{\text {pros }}$ DUB activity towards other substrates, we determined its ability to cleave differently-linked di-ubiquitin molecules. Co-incubation of $\mathrm{L}^{\text {pro }}$ with di-ubiquitin molecules of different linkages indicated that $\mathrm{L}^{\text {pro }}$ preferentially targets K6- and K48-linked ubiquitin chains, displayed some activity towards K11- , K33-, K63-, and M1-linked chains, but at this enzyme concentration and incubation times has no activity towards K27- or K29-linked ubiquitin chains (Fig 7). Neither mutation L143A nor C133S affected the ability of $\mathrm{L}^{\text {pro }}$ to cleave the di-ubiquitin molecules. Overall, our analysis shows that $\mathrm{L}^{\text {pro }}$ carrying mutation L143A or C133S has wt-like deISGylase and DUB activity. Importantly, as mutation Li43A impairs $\mathrm{L}^{\text {proos }}$ ability to cleave/degrade RLR signaling proteins (Fig 5), but does not affect its deISGylase/DUB activity, introduction of this mutation also allows us to make a distinction between the two proteolytic activities of $\mathrm{L}^{\mathrm{pro}}$.
Lys6 diUb

WT $C 133 S$ L143A $1 \mu \mathrm{M}$ $\mathrm{kDa} \quad \begin{array}{lllllllll}0 & 30 & 90 & 0 & 30 & 90 & 0 & 30 & 90 \\ \mathrm{~min}\end{array}$

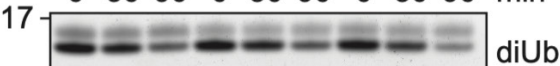
$6-$

\section{Lys27 diUb}

WT C133S L143A $1 \mu \mathrm{M}$ $\mathrm{kDa} \overline{03090} \overline{03090} \overline{03090} \mathrm{~min}$

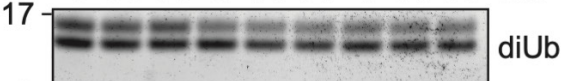

$6-$

\section{Lys33 diUb}

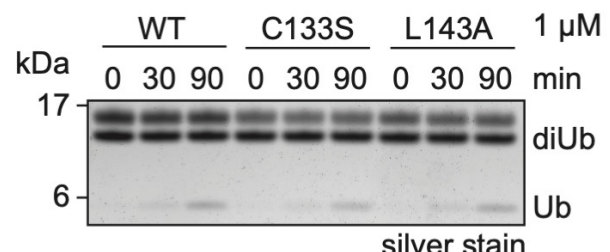

Lys63 diUb

WT C133S L143A $1 \mu \mathrm{M}$

kDa $\overline{03090} \overline{03090} \overline{03090} \mathrm{~min}$

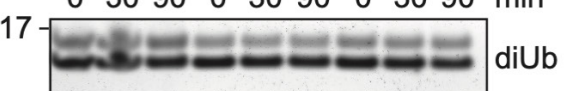

$6-$

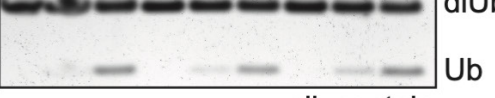

silver stain
Lys11 diUb

WT C133S L143A $1 \mu \mathrm{M}$ $\begin{array}{lllllllll} & 0 & 3090 & 0 & 3090 & 0 & 3090 & \text { min }\end{array}$

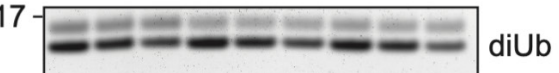
ニニーーーー- $\mathrm{diU}$ $-\ldots--\mathrm{U}$

Lys29 diUb

WT C133S L143A $1 \mu \mathrm{M}$

kDa $\quad 0 \quad 3090 \quad 0 \quad 3090 \quad 0 \quad 3090 \quad m i n$

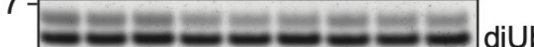

6

silver stain

Lys48 diUb

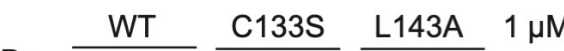

$\mathrm{kDa} \overline{03090} \overline{03090} \overline{03090} \mathrm{~min}$
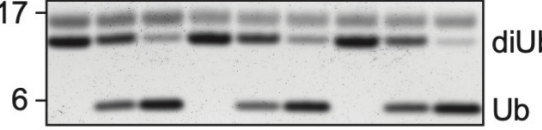

silver stain

Met1 diUb

WT $\mathrm{C} 133 \mathrm{~S}$ L143A $1 \mu \mathrm{M}$

$\begin{array}{lllllllllll}\mathrm{R} a & 0 & 30 & 90 & 0 & 30 & 90 & 0 & 30 & 90 & \mathrm{~min}\end{array}$

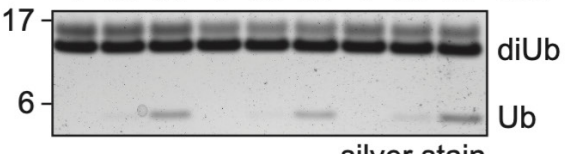

silver stain
Figure 7: Mutation of $L 143$ or $\mathrm{C} 133$ does not affect activity towards differently linked ubiquitin. Wild-type $\mathrm{L}^{\text {pro }}$ and the pro $^{\text {pro }}$ mutants ( $C_{133} S$, 1143A) were incubated with each of the eight di-ubiquitin chain types for the indicated times, separated by SDS-PACE, and visualized by silver-staining. The concentration of $L^{\text {pro }}$ used in the cleavage assays is indicated. 
$L^{\text {pro's }}$ ability to reduce IFN- $\beta$ mRNA levels correlates with its ability to cleave/degrade RLR signaling proteins, not with its deISGylase/DUB activity. Having characterized how the different mutations in $\mathrm{L}^{\text {pro }}$ affect its deISGylase/DUB activity or the cleavage/degradation of RLR proteins, we next set out to determine which mutants reduce the induction of IFN- $\beta$ mRNA. We infected HeLa cells with EMCV, EMCV- $\mathrm{L}^{\mathrm{Zn}}$ or the different EMCV- $\mathrm{L}^{\text {pro }}$ mutants and determined the IFN- $\beta$ mRNA and EMCV vRNA levels over time via RT-qPCR analysis (Fig 8). Wildtype $\mathrm{L}^{\text {pro }}$ as well as $\mathrm{L}^{\text {pro }} \mathrm{C} 133 \mathrm{~S}$, L92A and L102A consistently reduced the induction of IFN- $\beta$ mRNA, while $\mathrm{L}^{\text {pro }}$ C51A and L143A were unable to do so (Fig 8A). The SAP domain mutant (EMCV- $L^{\text {pro }}$ I83A/L86A) also failed to suppress IFN- $\beta$ mRNA levels (Fig 8B), which is in agreement with observations in FMDV-infected cells $(36,39)$. Notably, infection with EMCV $\mathrm{L}^{\text {pro }}$ L143A, which displayed wt deISGylase/DUB activity but is strongly impaired in its ability to cleave/degrade RLR signaling proteins MAVS, TBK1 and NFKB p65, failed to suppress the induction of IFN- $\beta$ mRNA. In contrast, $L^{\text {pro }}$ L92A and L102A, which are strongly impaired in their deISGylase/DUB activity (40) but not in their ability to cleave/degrade RLR signaling proteins, reduced IFN- $\beta$ mRNA levels. These combined observations (summarized in Fig 9) demonstrate that cleavage/degradation of RLR signaling proteins, but not the deISGylase/ DUB activity of $\mathrm{L}^{\mathrm{pro}}$, correlate with suppressing IFN- $\alpha / \beta$ gene transcription. Notably, $\mathrm{L}^{\text {pro }}$ C133S reduced the induction of IFN- $\beta$ mRNA despite a $2 \mathrm{~h}$ delay in eIF4 G cleavage, indicating that the rapid eIF4G cleavage and the subsequent translational shut-off is not sufficient to suppress RLR signaling.

\section{Discussion}

FMDV suppresses IFN- $\alpha / \beta$ both at the mRNA and at the protein level (47), but the molecular mechanism underlying the reduced induction of IFN- $\alpha / \beta$ gene transcription (RLR signaling) is poorly understood. Both the DUB activity of $\mathrm{L}^{\text {pro }}$ as well as its ability to cleave/degrade RLR signaling proteins have been implicated in the suppression of RLR signaling $(35,37,39)$. In this study, we identified MAVS and TBKi as novel $L^{\text {pro }}$ substrates and mapped the cleavage site in TBK1. Moreover, by introducing specific mutations we were able to separate $\mathrm{L}^{\text {pro's }}$ deISGylase/DUB activity from its ability to target RLR signaling proteins. Using $L^{\text {pro }}$ carrying either of these uncoupling mutations, we demonstrated that the cleavage/degradation of RLR signaling proteins, not the deISGylase/DUB activity, correlates with the ability to reduce IFN- $\beta$ gene transcription. Collectively, our data strongly suggest that the ability of $\mathrm{L}^{\text {pro }}$ to cleave/degrade RLR signaling proteins is needed to reduce the IFN- $\beta$ mRNA levels.
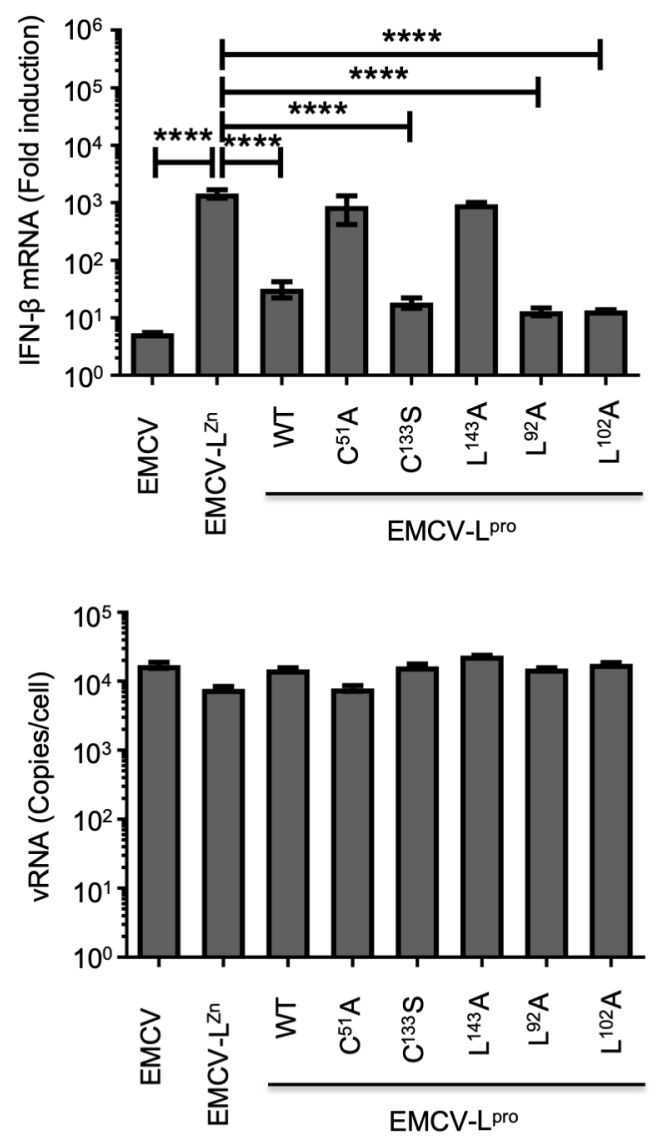

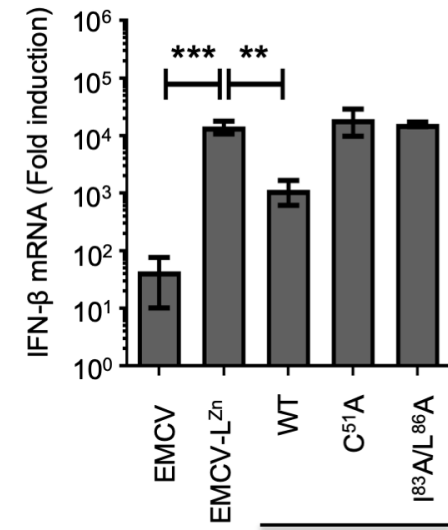

EMCV-Lpro

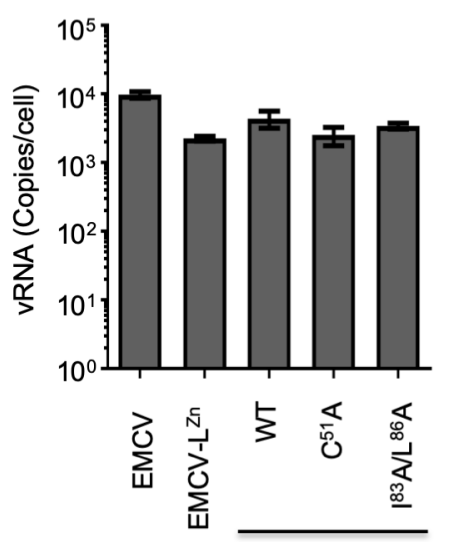

EMCV-Lpro
Figure 8: Reduction in IFN- $\beta$ gene transcription correlates with cleavage and/or degradation of RLR signaling proteins. (A+B) Hela R19 cells were infected at MOl 10 with the indicated viruses and cells were lysed at $8 \mathrm{hpi}$. Total RNA was isolated and used for RT- PCR anlysis for IFN $P$ and actin IFN- $\beta$ lod IFN- $\beta$ levels are depicted as a fold induction compared to levels in mock-infected cells, after correction for actin mRNA levels. The EMCV VRNA is depicted as a copy number per cell, calculated from a plasmid standard. Error bars represent the SD. One-way ANOVA with the Dunnet post hoc test was used to determine statistical significance compared to the

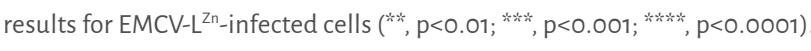

We identified TBKi as a new $\mathrm{L}^{\text {pro }}$ substrate and identified the cleavage site. We observed cleavage of TBKi both in HeLa cells infected with EMCV-L $\mathrm{L}^{\text {pro }}$ and in FMDV-infected LFPK cells, and we demonstrated that the $\mathrm{L}^{\text {pro }}$ cleavage site is located towards the $\mathrm{C}$ terminus of $\mathrm{TBK}$, more specifically in the coiled-coil 2 (CC2) domain. Previous work indicated that TBK1 $\triangle \mathrm{CC} 2$ was signaling competent upon overexpression of TBK1. However, it was also shown that mutation of residues L693 and K694, which are located in the CC2 domain, abolishes IFN- $\beta$ mRNA induction upon polyI:C transfection and VSV infection (50). $\mathrm{L}^{\text {pro }}$ cleaves TBK1 at these residues. Yet, we observed that the N-terminal cleavage product restored RLR signaling in TBKi k.o. cells 


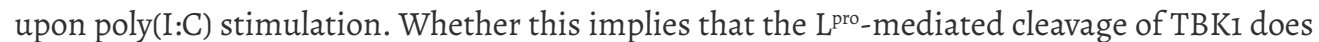
not contribute to the viral strategy to suppress RLR signaling and IFN- $\beta$ gene transcription remains unknown. Unfortunately, it is very difficult to investigate the effect of TBKi cleavage by $\mathrm{L}^{\text {pro }}$ on IFN- $\beta$ gene transcription in infected cells, as $\mathrm{L}^{\text {pro }}$ also cleaves other RLR signaling proteins, i.e. MAVS (this study) and LGP2 ((51), see below), and the transcription factor NF$\kappa B$. Dissection of the effect of TBKi cleavage on IFN- $\beta$ gene transcription from the effect of MAVS, LGP2 and NF- $\mathrm{KB}$ cleavage would require the identification of all $\mathrm{L}^{\text {pro }}$ cleavage sites in these known targets followed by the generation of cells with cleavage-resistant versions of these proteins. It remains a question whether such an approach will yield conclusive answers as other RLR signaling proteins may be targeted by $L^{\text {pro }}$, which have not yet been identified. Hence, the relative importance of the $\mathrm{L}^{\text {pro }}$-mediated cleavage of TBKI for the viral suppression of IFN- $\beta$ gene transcription remains unknown.

Apart from its role in RLR signaling, TBKi has been suggested to be involved in autophagosome maturation. TBKI was identified as a factor in the autophagosomal clearance of herpes simplex virus 1 and mycobacteria $(52,53)$. A recent report showed that TBK1 phosphorylates lipidated $\mathrm{LC}_{3}$ to prevent premature removal of $\mathrm{LC}_{3}$ from autophagosomal membranes by ATG4, thereby facilitating a unidirectional flow from the autophagosome to the lysosome (54). Many picornaviruses hijack autophagic pathways to generate sites for viral RNA replication and to facilitate non-lytic release of virions (55-61) Possibly, cleavage of TBKi by L $L^{\text {pro }}$ facilitates the use of autophagy to aid viral infection and propagation.

$L^{\text {pro }}$ also impacts MAVS integrity. A distinct MAVS cleavage product was observed upon infection

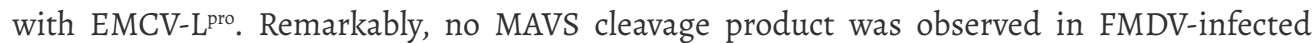
cells, although MAVS levels progressively declined over time. The reason for this difference is unknown, but may be related to differences in human and porcine cells. Nevertheless, our data indicate that the integrity of MAVS is affected by $\mathrm{L}^{\text {pro }}$ in both cell types, suggesting this RLR signaling protein is targeted by FMDV to affect IFN induction. Of note, MAVS is known to localize to the peroxisomes and mitochondrion associated membranes of the ER $(62,63)$, in addition to its default localization on mitochondria. It remains to be established whether $L^{\text {pro }}$ targets all forms of MAVS or specifically targets MAVS at one of these locations. TBKI and MAVS are not the only RLR signaling proteins that are targeted by $\mathrm{L}^{\text {pro. }}$. It was previously reported that overexpression of $\mathrm{L}^{\text {pro }}$ induces the degradation of IRF3 and IRF7 (37), and that the p65 subunit of NF- $\mathrm{KB}$ is degraded in FMDV-infected cells (35). We also observed degradation of NF-KB p65 in EMCV-L $\mathrm{L}^{\text {pro }}$ infected cells but we did not observe degradation of IRF3. Notably, degradation of IRF3 was also not observed in FMDV-infected cells. Whether IRF3 degradation is restricted to certain cell types or conditions, or merely is an artefact due to overexpression remains unknown.

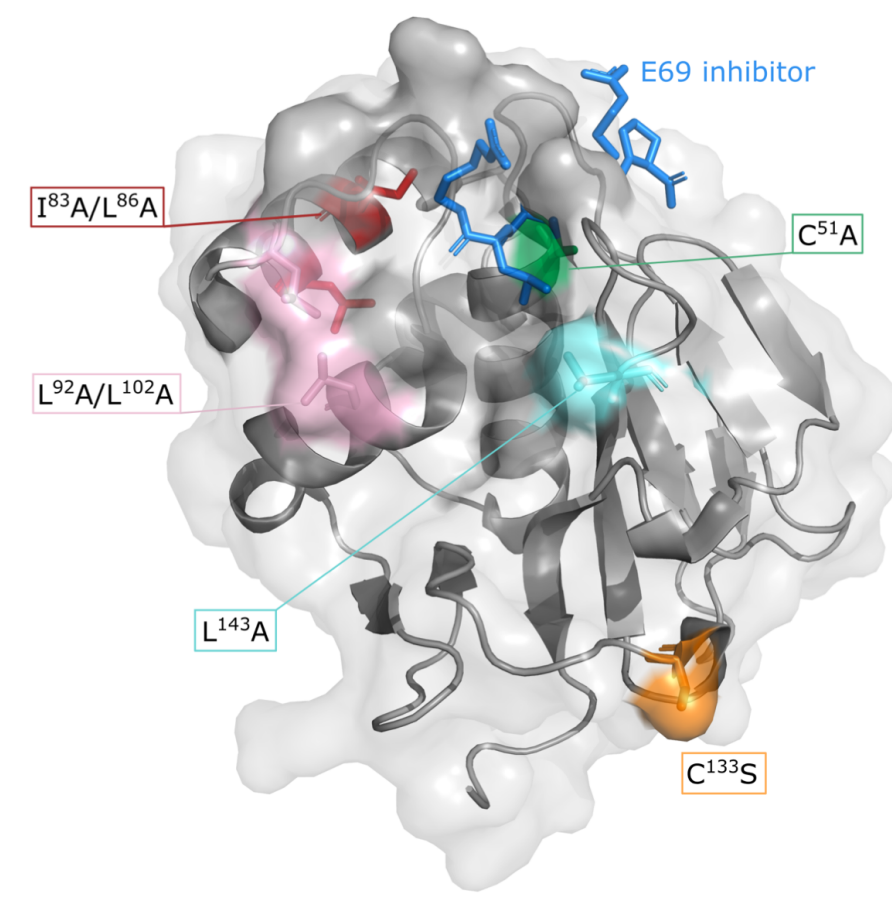

B

\begin{tabular}{|c|c|c|c|c|c|c|}
\hline & $\begin{array}{c}\text { elF4G } \\
\text { cleavage }\end{array}$ & $\begin{array}{c}\text { G3BP1 } \\
\text { cleavage }\end{array}$ & $\begin{array}{c}\text { TBK1 and MAVS } \\
\text { cleavage }\end{array}$ & $\begin{array}{c}\text { NFkB } \\
\text { degradation }\end{array}$ & $\begin{array}{c}\text { deISGylase } \\
\text { / DUB act. }\end{array}$ & $\begin{array}{c}\text { Suppression } \\
\text { IFN- } \beta \text { levels }\end{array}$ \\
\hline $\mathrm{Lpro}^{2}$ & ++ & ++ & ++ & ++ & ++ & ++ \\
\hline${ }^{51} \mathrm{~A}$ & - & - & - & - & - & - \\
\hline${ }^{83} \mathrm{~A} / \mathrm{L}^{86} \mathrm{~A}$ & ++ & + & $+/-$ & - & - & - \\
\hline${ }^{{ }^{292} \mathrm{~A}}$ & ++ & ++ & ++ & ++ & $+/-$ & ++ \\
\hline $\mathrm{L}^{102 \mathrm{~A}}$ & ++ & ++ & ++ & n.d. & $+/-$ & ++ \\
\hline${ }^{133 \mathrm{~S}}$ & + & ++ & ++ & ++ & ++ & ++ \\
\hline${ }^{1433} \mathrm{~A}$ & + & + & $+/-$ & - & ++ & - \\
\hline
\end{tabular}

Figure 9: Overview of the effects of $L^{\text {pro }}$ mutations on the different proteolytic activities of $L^{\text {pro }}$ as well as reduction in IFN- $\beta$ gene transcription. (A) Standard cartoon view with transparent surface of L pro bound to E69 inhibitor shown as blue sticks (PDB: $4 Q B B)$. Residues which upon mutation were reported to affect $L^{\text {prows }}$ structure or function are shown as colored sticks. Green: C51A inactivates Lpros catalytic activity; red: 183A or L86A reduce the DUB activity and IFN induction; pink: L92A and L102A reduce affinity for ISG15; orange: $\mathrm{C}_{133} \mathrm{~S}$ reduces affinity for elF4G; aquamarine: L143A predicted to open substrate binding pocket. Drawings were generated using PyMol. (B) Overview of the effects of introduced mutations on cleavage and/or degradation of host proteins, deISCylase/DUB activity, and their ability to reduce IFN- $\beta$ gene transcription. Coloring of mutations is consistent with panel $\mathrm{A}$. The activities of the mutations have been scored,,$++++/-$, or - according to the following criteria. ++ , activity is similar to wt L Lro, + , moderately reduced activity; + /-, severely impaired activity; - , no activity 
It is remarkable that $\mathrm{L}^{\text {pro }}$, comprising just 173 amino acids, can carry out several specific proteolytic activities on both the viral and cellular substrates as summarized in Figure 4 and 9. Previous work documented areas of the protease that are required for polyprotein processing $(41,49)$. These residues included L143 which is part of the P2 pocket that can interact with leucine residues in the substrate at the P2 position. In this work, L143 was identified as being involved in TBKi and MAVS cleavage; however, its replacement by alanine affected neither the activity on eIF4G nor the deISGylase or DUB activities. Surprisingly, mutation of two residues of the SAP domain, (I83 and L86) also affected TBKi and MAVS cleavage, even though they are separated by $20 \AA$ (measured between the respective $\mathrm{C}_{\alpha}$ atoms) with helix $\alpha 4$ lying between them. Nevertheless, it cannot be excluded that the SAP mutations cause some destabilization of $\mathrm{L}^{\text {pro }}$, thereby explaining this mutant's defect in proteolytic activities. Further structural work will be required to understand how $\mathrm{L}^{\text {pro }}$ interacts with TBKi and MAVS.

While this work was in progress, it was reported that LGP2, a factor that is essential for MDA5 activation, is cleaved by $L^{\text {pro }}(51)$. The mutations in $L^{\text {pro }}$ that impaired the reduction of IFN- $\beta$ mRNA levels (L143A and mutations in the SAP domain) displayed an overall defect in the cleavage and/or degradation of each of the RLR signaling proteins we studied (i.e. MAVS, TBK1 and NF- $\mathrm{KB}$ р65). We anticipate that the cleavage of LGP2 is also likely impaired by introduction of these mutations. Our data suggest that expression of $L^{\text {pro }}$ results in cleavage and/or degradation of multiple RLR signaling proteins (MAVS, TBKI, NF- $\kappa B$ p65, and most likely LGP2). The relative contribution of each cleavage event to the reduction in IFN- $\beta$ gene transcription remains unknown. A search for other substrates of $\mathrm{L}^{\text {pro }}$ is of importance to further our understanding of the role and mechanism of how $\mathrm{L}^{\text {pro }}$ reduces IFN- $\alpha / \beta$ induction. Possibly, such a search may identify RLR signaling proteins that are cleaved earlier than the ones identified so far and may thereby have an influence on the early induction of type I IFN in infected cells.

The $\mathrm{L}^{\text {pro }}$ mutants that are defective in either the deISGylase/DUB activity or the cleavage/ degradation of RLR signaling proteins allowed us to study which ability is needed to suppress RLR signaling. Mutation L143A, which rendered $L^{\text {pro }}$ unable to reduce IFN- $\beta$ mRNA levels, impaired the cleavage of RLR signaling molecules, but had no effect on the deISGylase or DUB activity of $\mathrm{L}^{\text {pro }}$. Meanwhile, mutations L92A and L102A resulted in the opposite phenotypic effect; RLR signaling proteins were cleaved with similar efficiency as wt $\mathrm{L}^{\text {pro, }}$, but the deISGylase activity was significantly reduced by these mutations (40). Yet, these $\mathrm{L}^{\text {pro }}$ mutants still reduced IFN- $\beta$ mRNA levels. Collectively, these data indicate that the activity of $L^{\text {pro }}$ to cleave/degrade RLR signaling proteins, not its deISGylase/DUB activity, is important for reduction of IFN- $\beta$ induction. In support of this hypothesis, Medina et. al. have just reported that impairment of the deISGylation activity of $\mathrm{L}^{\text {pro }}$ causes viral attenuation in vitro and in vivo, without affecting IFN or ISG mRNA expression levels (64).
It was previously suggested that the DUB activity of $\mathrm{L}^{\text {pro }}$ is important for the suppression of RLR signaling (39), which contrasts our findings. Importantly, most experiments by Wang et. al. relied on overexpression of $\mathrm{L}^{\text {pro }}$, ubiquitin, and several targets proteins (i.e. RIG-I, TBK1, TRAF3 and TRAF6). A recent study showed that $\mathrm{L}^{\text {pro }}$ should be predominantly regarded as a deISGylase rather than a DUB, as biochemical evidence showed that $\mathrm{L}^{\text {pro }}$ has a 1000 -fold higher activity towards ISG15 than ubiquitin (40). Given the weak DUB activity of $\mathrm{L}^{\text {pro }}$ in vitro, it remains to be established whether $\mathrm{L}^{\text {pro }}$ genuinely acts as a DUB in FMDVinfected cells under physiological conditions (i.e. without overexpression of components of the ubiquitination system or known ubiquitination target proteins). Previously, we found no differences in the levels of ubiquitinated proteins in cells infected with EMCV expressing $w t \mathrm{~L}^{\text {pro }}$ or $\mathrm{L}^{\text {pro }} \mathrm{C} 5 \mathrm{~A} \mathrm{~A}$, whereas the levels of ISGylated protein were decreased in cells infected with EMCV-L $\mathrm{L}^{\text {pro }}(40)$, suggesting that $\mathrm{L}^{\text {pro }}$ predominantly acts as a deISGylase in infected cells.

It is well established that certain viruses (i.a. adenoviruses, herpesviruses and nidoviruses) rely on viral proteases with DUB and deISGylase activity to suppress the induction of IFN- $\alpha / \beta$ (9). FMDV $L^{\text {pro }}$ is a papain-like protease and thus $L^{\text {pro }}$ is best compared to other virally encoded papain-like cysteine proteases that suppress IFN- $\alpha / \beta$ gene transcription. Members of the order Nidovirales (i.e. coronaviruses and arteriviruses) encode one or two papain-like cysteine protease (PLP), referred to as PLro, or PLP1 and PLP2 when the virus encodes two PLPs. In addition to cleaving the viral polyprotein, $\mathrm{PL}^{\mathrm{pro}}$ and the equivalent PLP2 have acquired DUB and deISGylase activity (65-73). Structure-guided mutagenesis of $\mathrm{PL}^{\text {pro }}$ of MERS-CoV and PLP2 of equine arterivirus (EAV) allowed the DUB activity to be separated from the proteolytic activity portrayed towards the viral polyprotein $(72,73)$. This uncoupling of these two different proteolytic activities indicated that the DUB activity of $\mathrm{PL}^{\mathrm{pro}} / \mathrm{PLP} 2$ contributes to the suppression of IFN- $\alpha / \beta$ transcription $(72,73)$. Unfortunately, it has not been determined whether PLro/PLP2 cleaves RLR signaling proteins and thus it is unclear what other proteolytic activities could be affected by the mutations that were introduced. Notably, the cleavage site of nidovirus PL pro $/ \mathrm{PLP} 2$ and FMDV $\mathrm{L}^{\text {pro }}$ in ubiquitin and ISG15 is different. While SARS-CoV PL pro breaks the iso-peptide bond between ubiquitin or ISG15 and the target protein $(65,66), F M D V L^{\text {pro }}$ is a non-canonical deISGylase that targets a peptide bond in ISG15 itself, resulting in a diglycylated-lysine in the target protein (40). In conclusion, nidovirus PL ${ }^{\text {pro }} /$ PLP2 and FDMV L ${ }^{\text {pro }}$ are both papain-like proteases, but they have evolved different strategies to suppress IFN- $\alpha / \beta$ gene transcription.

FMDV $L^{\text {pro }}$ and enterovirus $2 \mathrm{~A}^{\text {pro }}$ are structurally different enzymes that share many functions; both cleave translation initiation factor $\operatorname{eIF} 4 \mathrm{G}(34,74,75)$, both reduce IFN$\alpha / \beta$ gene transcription $(27,47,76,77)$, both have been implicated in the suppression of SG formation (77-79) and both have been suggested to rescue viral translation from the 
inhibitory effects of p-eIF2 $(80,81)$. Importantly, $\mathrm{L}^{\text {pro }}$ and $2 \mathrm{~A}^{\text {pro }}$ both cleave several RLR signaling proteins, but the only overlapping RLR protein is MAVS $(27,76)$. Although a causal relationship between cleavage of RLR proteins and suppression of IFN- $\alpha / \beta$ transcription remains to be established for both proteases, the convergence on the cleavage of MAVS is noteworthy. In the absence of sequence homology, no evolutionary basis for the functional similarities between the two picornavirus proteases can be determined. Possibly, the extensive similarities between FMDV L $\mathrm{L}^{\text {pro }}$ and enterovirus $2 \mathrm{~A}^{\text {pro }}$, both picornavirus proteases, is illustrative of the urgency for picornaviruses to suppress these particular antiviral host responses.

Our data suggests that the deISGylase activity of $\mathrm{L}^{\text {pro }}$ is not critically needed to suppress RLR signaling, but rather its role should be sought in the broader antiviral activities of ISG15 (reviewed in (10, 11)). It should be noted that our experiments were performed in naive cells at high multiplicity of infection. Expression of the ISGylation machinery as well as ISG15 itself are boosted by IFN- $\alpha / \beta$ and therefore we cannot formally exclude a role for the DUB and/or deISGylase activity of $\mathrm{L}^{\text {pro }}$ in suppressing RLR signaling under different conditions (e.g. in IFN-primed cells). ISG15 has many functions, both intracellular and extracellular. Intracellular ISG15 can act both pro-inflammatory and immunomodulatory, either via ISGylation of target proteins or as free ISG15. Moreover, ISG15 can be secreted to act as a cytokine $(25,26)$. ISG15 also plays a role in damage repair after clearing viral infection (82) and can regulate cellular processes such as autophagy and metabolism (24, 83-85). How the deISGylase activity of $\mathrm{L}^{\text {pro }}$ contributes to efficient in vivo infection, remains to be established.

\section{Materials and Methods}

Cells and viruses. HeLa R19, HeLa R19 TBKi k.o. and Hek293T cells were maintained in Dulbecco's Modified Eagle's Medium (DMEM) supplemented with 10\% FCS (V/V). HeLa OHIO cells were maintained in DMEM supplemented with 10\% FCS (V/V) and $1 \%$ penicillin/streptomycin. LFPK $\alpha v \beta 6$ cells (86) were obtained from the Foreign Animal Disease Diagnostic Laboratory (FADDL) at the PIADC. These cells were maintained in minimal essential medium (MEM) supplemented with $10 \%$ FCS $(\mathrm{V} / \mathrm{V})$ and $1 \%$ antibiotics and non-essential amino acids. BHK-21 cells used for FMDV propagation were maintained in MEM supplemented with $10 \%$ FCS (V/V), 10\% tryptose phosphate broth, $1 \%$ antibiotics and non-essential amino acids. HeLa R19 TBKi k.o. cells were generated via CRISPR/cas9 methodology using a pCRISPR plasmid, as described previously (87). The used gRNA sequences are 5'-GCTACTGCAAATGTCTTTCG-3' and 5'-GAGGAAAACAGATTGGTT-3'. FMDV A12-WT (wild type) was generated from the full-length serotype A12 infectious clone,
pRMC35 (88) and A12-LLV2 (leaderless virus) was derived from the infectious clone lacking the Lb coding region, PRM-LLV2 (89). Viruses were propagated in BHK-21 and concentrated by polyethylene glycol precipitation, titrated on BHK-21 cells, and stored at $-70^{\circ} \mathrm{C}$. ERAV (NM-11/67 strain) (gift from D. Rowlands and T. Tuthill (90)) was obtained after passage on HeLa R19 cells and subsequently concentrated by ultracentrifugation through a $30 \%$ sucrose cushion at $140,000 \mathrm{xg}$ for 16 hours in a SW32Ti rotor and stored at $-80^{\circ} \mathrm{C}$. Recombinant EMCVs were generated by cloning the genes of interest into the XhoI/NotI restriction sites from the PM16.I-VFETQG-Zn infectious clone that was described previously (45). EMCV$\mathrm{L}^{\text {pro }}$ viruses were recovered by transfection of run-off RNA transcripts into BHK-21 cells. Upon total CPE, viruses were concentrated by ultracentrifugation (as described for ERAV) and stored at $-80^{\circ} \mathrm{C}$.

Antibodies. The following antibodies were used for Western blot staining procedures: $\alpha F M D V$ VP1 (rabbit polyclonal Abmade at PIADC), $\alpha E M C V$ capsid (gift from Ann Palmenberg), $\alpha L^{\text {pro }}$ (gift from Ewald Beck and Tim Skern), $\alpha$ MAVS (Enzo life science ALX-

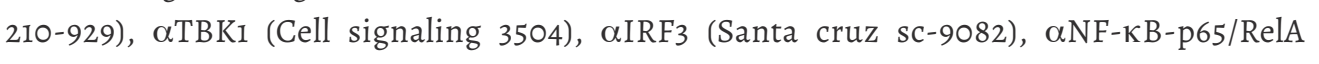
(Santa Cruz Biotechnology SC-8008), aeIF4G (Bethyl laboratories A300-502A), $\alpha$ G3BP1 (BD biosciences clone 23/G3BP), $\alpha$ PARP (Roche Diagnostics \#11835238001), $\alpha$ Flag (Sigma M2), $\alpha$ GFP (Invitrogen OSE00003G), $\alpha$ His (GE Healthcare, 27-4710-01), $\alpha$ Myc (Clone 4A6, Millipore), $\alpha \mathrm{HA}$ (Abcam ab130275) and $\alpha$ tubulin (Sigma DM1A). Respective IRdye680 or IRdye800 conjugated secondary antibodies (LiCOR) or HRP-conjugated secondary antibodies were used for detection.

RT-PCR analysis. HeLa R19 cells were seeded in 24-wells plates and the next day infected with the indicated viruses at MOI 10 or transfected with the indicated plasmids or vRNA. Plasmids were transfected using Fugene6 (Promega) and vRNA was transfected using Lipofectamine 2000 (Invitrogen), both according to the manufacturer's instructions. Preparation of viral dsRNA and the pcDNA-GFP-MAVS construct have been described previously $(1,44)$. pEGFP-IRF3 [D5] construct was a kind gift from John Hiscott (91). At the indicated time points cells were lysed and cellular RNA was isolated using total RNA isolation kit (Machery-Nagel) according to manufacturer's instructions. Reverse transcription was set up using TaqMan Reverse Transcription Reagents (Applied Biosystems) before performing qPCR analysis with SYBR green (Roche) as described previously (92).

Western Blot analysis of transfected cells. The pIRES-EGFP-FMDV $L^{\text {pro }}$ plasmid was described previously (45). The pcDNA-Flag-TBK1 plasmid was a gift from John Hiscott (93) and the pEF-FLAG-RIG-I was a gift from Takashi Fujita (94). HA-ubiquitin was expressed from a pCMV5 plasmid. Hek293T cells were seeded in 6-well plates and the next day transfected with $1.5 \mu \mathrm{g}$ of total plasmid using Fugene6 (Promega) according to 
manufacturer's instructions. 16h posttransfection cells were lysed $100 \mu \mathrm{l}$ lysisbuffer (100 mM Tris pH 8.0, 1 mM EDTA, $50 \mathrm{mM} \mathrm{NaCl}, 1 \% \mathrm{NP} 40$, protease inhibitor mix (Roche)). Post nuclear lysate was obtained by centrifugation at $15000 x g$ at $4{ }^{\circ} \mathrm{C}$ for $15 \mathrm{~min}$. The amount of total protein in the lysates was determined using BCA assay (ThermoFisher) and 100 $\mu \mathrm{g}$ of protein was resolved using reducing sodiumdodecyl sulfate-polyacrylamide gel electrophoresis (SDS-PAGE) and transferred to $0.2 \mu \mathrm{m}$ nitrocellulose membranes by wet electrophoretic transfer. Membranes were incubated ih in blocking buffer (PBS $+0.1 \%$ Tween $20+2 \%$ BSA) and successively incubated overnight with primary antibodies diluted in blocking buffer and then for $30 \mathrm{~min}$ with respective secondary antibodies diluted in blocking buffer. Between and after the incubations, the membranes were washed three times with PBS+0.1\% Tween-20. Finally, membranes were washed once with PBS and scanned using an Odyssey Imager (Li-COR).

Western Blot analysis of infected cells. HeLa R19 cells were seeded in $10 \mathrm{~cm}$ dishes and infected the next day with the indicated viruses at MOI 10. At the indicated time points cells were released using trypsin, washed once in PBS and lysed in $100 \mu$ l lysis buffer (100 mM Tris pH 8.0, I mM EDTA, $50 \mathrm{mM} \mathrm{NaCl}, 1 \% \mathrm{NP} 40$, protease inhibitor mix (Roche)). Subsequent steps are identical as described for transfected cells. For the analysis of FMDV-infected LFBK $\alpha v \beta 6$ cultures, cells were lysed in lysis buffer (0.5\% NP-40 substitute, $50 \mathrm{mM}$ Tris $\mathrm{pH}$ $7.5,150 \mathrm{mM} \mathrm{NaCl}, 1 \mathrm{mM} \mathrm{EDTA})$. Lysates were incubated at $4^{\circ} \mathrm{C}$ for $10 \mathrm{~min}$ and cellular debris was collected by centrifugation at 10,000xg for $15 \mathrm{~min}$ at $4^{\circ} \mathrm{C}$. $40 \mathrm{ng}$ of protein was resolved by SDS-PAGE, transferred by Western blot and secondary antibodies conjugated with horseradish peroxidase (Pierce) were used for detection of proteins. Following incubation with appropriate primary and secondary antibodies, protein bands were visualized using SuperSignal ${ }^{\circledR}$ West Dura Extended Duration Substrate (ThermoScientific, Rockford, IL, USA) according to the manufacturer's directions.

In vitro TBK1 cleavage. $\mathrm{sL}^{\text {pro }}$ was expressed and purified as reported previously (95). $275 \mathrm{ng}$ of His-hTBKI (Millipore) was incubated with $0-3 \mu \mathrm{g}$ sL pro for $2 \mathrm{~h}$ at $30^{\circ} \mathrm{C}$ in a HEPES buffer (20 mM HEPES pH 7.4, $150 \mathrm{mM} \mathrm{KCl,} 1 \mathrm{mM}$ EDTA) before the reaction mixture was dissolved on SDS-PAGE. Proteins were transferred to nitrocellulose and Western blot staining for the his-tag was performed. Myc-mTBK1 and Myc- $\mathrm{mTBK}_{692 \mathrm{AAA} 694}$ were transiently expressed in HeLa OHIO cells from plasmid pCS2-6Myc-mTBK1, a gift from T. Decker. $20 \mu \mathrm{g}$ myctagged mTBKi containing cell lysate was incubated with $2 \mu \mathrm{g}$ sL pro for $2 \mathrm{~h}$ at $30^{\circ} \mathrm{C}$ in a HEPES buffer before resolving the reaction mixture on SDS-PAGE, transferring the protein to nitrocellulose membrane and performing Western blot staining for Myc.
In vitro DUB and deISGylase assays. Ubiquitin/ISG15-TAMRA assays were performed according to (96). Di-ubiquitin in vitro cleavage assays were performed as described previously (97).

\section{Acknowledgments}

This work was supported by a Vici grant from the Netherlands Organization for Scientific Research (NWO-918.12.628) and the European Union (Horizon 2020 Marie SkłodowskaCurie ETN "INITIATE", grant agreement number 813343). LJV was supported by the Graduate Programme Infection and Immunity (NWO-022.004.018) and MAL was supported by a Veni grant (NWO-863.13.008), both from the Netherlands Organization for Scientific Research. GNM and TDLS were supported by USDA-ARS, CRIS project number 1940-32000-061-00D, and USDA-KSU Non-Assistance Cooperative Agreement \#588064-8-010. TS and KMO were supported by grants P28183 and W1258 from the Austrian Science Foundation. KNS and DK were supported by the Medical Research Council (Grant U105192732) the European Research Council (Grants 309756 and 724804), and the Lister Institute for Preventive Medicine. 


\section{References}

1. Feng Q, Hato SV V., Langereis MAA, Zoll J, Virgen-Slane R, Peisley A, Hur S, Semler BLL, van Rij RP, van Kuppeveld FJM. 2012. MDA5 Detects the Double-Stranded RNA Replicative Form in Picornavirus-Infected Cells. Cell Rep 2:1187-1196.

2. Wang JP, Cerny A, Asher DR, Kurt-Jones EA, Bronson RT, Finberg RW. 2010. MDA5 and MAVS mediate type I interferon responses to coxsackie B virus. J Virol 84:254-60.

3. Kuo R-L, Kao L-T, Lin S-J, Wang RY-L, Shih S-R. 2013. MDA5 Plays a Crucial Role in Enterovirus 71 RNAMediated IRF3 Activation. PLoS One 8:e63431.

4. Hüsser L, Alves MP, Ruggli N, Summerfield A. 2011. Identification of the role of RIG-I, MDA-5 and TLR3 in sensing RNA viruses in porcine epithelial cells using lentivirus-driven RNA interference. Virus Res 159:9-16.

Ivashkiv LB, Donlin LT. 2014. Regulation of type I interferon responses. Nat Rev Immunol 14:36-49.

6. Goubau D, Deddouche S, Reis e Sousa C. 2013. Cytosolic Sensing of Viruses. Immunity 38:855-869.

7. Chiang C, Gack MU. 2017. Post-translational Control of Intracellular Pathogen Sensing Pathways. Trends Immunol 38:39-52.

8. Heaton SM, Borg NA, Dixit VM. 2016. Ubiquitin in the activation and attenuation of innate antiviral mmunity. J Exp Med 213:1-13.

9. Bailey-Elkin BA, Knaap RCM, Kikkert M, Mark BL. 2017. Structure and Function of Viral Deubiquitinating Enzymes. J Mol Biol 429:3441-3470.

10. Perng Y-C, Lenschow DJ. 2018. ISG15 in antiviral immunity and beyond. Nat Rev Microbiol 16:423-439.

11. Dzimianski J V., Scholte FEM, Bergeron É, Pegan SD. 2019. ISG15: it’s Complicated. J Mol Biol Sooz22836:30136-6.

12. Lai C, Struckhoff JJ, Schneider J, Martinez-Sobrido L, Wolff T, García-Sastre A, Zhang D-E, Lenschow DJ. 2009. Mice Lacking the ISG15 E1 Enzyme UbEIL Demonstrate Increased Susceptibility to both MouseAdapted and Non-Mouse-Adapted Influenza B Virus Infection. J Virol 83:1147-1151.

13. Ketscher L, Hannß R, Morales DJ, Basters A, Guerra S, Goldmann T, Hausmann A, Prinz M, Naumann R, Pekosz A, Utermöhlen O, Lenschow DJ, Knobeloch K-P. 2015. Selective inactivation of USP18 isopeptidase activity in vivo enhances ISG15 conjugation and viral resistance. Proc Natl Acad Sci 112:1577-1582.

14. Rahnefeld A, Klingel K, Schuermann A, Diny NL, Althof N, Lindner A, Bleienheuft P, Savvatis K, Respondek D, Opitz E, Ketscher L, Sauter M, Seifert U, Tschöpe C, Poller W, Knobeloch K-P, Voigt A. 2014. Ubiquitin-like protein ISG15 (interferon-stimulated gene of $15 \mathrm{kDa}$ ) in host defense against heart failure in a mouse model of virus-induced cardiomyopathy. Circulation 130:1589-600.

15. Lenschow DJ, Lai C, Frias-Staheli N, Giannakopoulos N V., Lutz A, Wolff T, Osiak A, Levine B, Schmidt RE, García-Sastre A, Leib DA, Pekosz A, Knobeloch KP, Horak I, Virgin IV HW. 2007. IFN-stimulated gene 15 functions as a critical antiviral molecule against influenza, herpes, and Sindbis viruses. Proc Natl Acad Sci U S A 104:371-6.

16. Daczkowski CM, Dzimianski J V., Clasman JR, Goodwin O, Mesecar AD, Pegan SD. 2017. Structural Insights into the Interaction of Coronavirus Papain-Like Proteases and Interferon-Stimulated Gene Product 15 from Different Species. J Mol Biol 429.

17. Bogunovic D, Byun M, Durfee LA, Abhyankar A, Sanal O, Mansouri D, Salem S, Radovanovic I, Grant A V., Adimi P, Mansouri N, Okada S, Bryant VL, Kong XF, Kreins A, Velez MM, Boisson B, Khalilzadeh S, Ozcelik U, Darazam IA, Schoggins JW, Rice CM, Al-Muhsen S, Behr M, Vogt G, Puel A, Bustamante J, Gros P, Huibregtse JM, Abel L, Boisson-Dupuis S, Casanova JL. 2012. Mycobacterial disease and impaired IFN- $\gamma$ immunity in humans with inherited ISG15 deficiency. Science (80- ) 337:1684-8.
18. Zhang X, Bogunovic D, Payelle-Brogard B, Francois-Newton V, Speer SD, Yuan C, Volpi S, Li Z, Sanal O, Mansouri D, Tezcan I, Rice GI, Chen C, Mansouri N, Mahdaviani SA, Itan Y, Boisson B, Okada S, Zeng L Wang X, Jiang H, Liu W, Han T, Liu D, Ma T, Wang B, Liu M, Liu J-Y, Wang OK, Yalnizoglu D, Radoshevich Wa Uze G, Gros P, Rozenberg F, Zhang S-Y, Jouanguy E, Bustamante J, García-Sastre A, Abel L, Lebon P, Notarangelo LD, Crow YI, Boisson-Dupuis S, Casanova I-L, Pellegrini S. 2015. Human intracellular ISG15 prevents interferon- $\alpha / \beta$ over-amplification and auto-inflammation. Nature 517:89-93.

19. Speer SD, Li Z, Buta S, Payelle-Brogard B, Qian L, Vigant F, Rubino E, Gardner TJ, Wedeking T, Hermann M, Duehr J, Sanal O, Tezcan I, Mansouri N, Tabarsi P, Mansouri D, Francois-Newton V, Daussy CF, Rodriguez MR, Lenschow DJ, Freiberg AN, Tortorella D, Piehler J, Lee B, García-Sastre A, Pellegrini S, Bogunovic D. 2016. ISG15 deficiency and increased viral resistance in humans but not mice. Nat Commun 7:11496.

20. Durfee LA, Lyon N, Seo K, Huibregtse JM. 2010. The ISG15 Conjugation System Broadly Targets Newly Synthesized Proteins: Implications for the Antiviral Function of ISG15. Mol Cell 38:722-32.

21. Shi HX, Yang K, Liu X, Liu XY, Wei B, Shan YF, Zhu LH, Wang C. 2010. Positive Regulation of Interferon Regulatory Foctor 3 Activation by Hercs via ISG15 Modfication. MelCell Biol $302424-36$.

22. Ganesan M, Poluektova LY, Tuma DJ, Kharbanda KK, Osna NA. 2016. Acetaldehyde Disrupts Interferon Alpha Signaling in Hepatitis C Virus-Infected Liver Cells by Up-Regulating USP18. Alcohol Clin Exp Res 40:2329-2338.

23. Kim M-J, Hwang S-Y, Imaizumi T, Yoo J-Y. 2008. Negative Feedback Regulation of RIG-I-Mediated Antiviral Signaling by Interferon-Induced ISG15 Conjugation. J Virol 82:1474-83.

24. Du Y, Duan T, Feng Y, Liu Q, Lin M, Cui J, Wang R. 2018. LRRC25 inhibits type I IFN signaling by targeting ISG15-associated RIG-I for autophagic degradation. EMBO J 37:351-366.

25. Bogunovic D, Boisson-Dupuis S, Casanova JL. 2013. ISG15: Leading a double life as a secreted molecule. Exp Mol Med.

26. Swaim CD, Scott AF, Canadeo LA, Huibregtse JM. 2017. Extracellular ISG15 Signals Cytokine Secretion through the LFA-1 Integrin Receptor. Mol Cell.

27. Feng Q, Langereis MA, Lork M, Nguyen M, Hato S V, Lanke K, Emdad L, Bhoopathi P, Fisher PB, Lloyd RE, van Kuppeveld FJM. 2014. Enterovirus 2Apro targets MDA5 and MAVS in infected cells. J Virol 88:3369-78.

28. Qian S, Fan W, Liu T, Wu M, Zhang H, Cui X, Zhou Y, Hu J, Wei S, Chen H, Li X, Qian P. 2017. Seneca Valley Virus Suppresses Host Type I Interferon Production by Targeting Adaptor Proteins MAVS, TRIF, and TANK for Cleavage. J Virol 91:e00823-17.

29. Zhang H-L, Ye H-Q Liu S-Q Deng C-L, Li X-D, Shi P-Y, Zhang B. 2017. West Nile Virus NSi Antagonizes Interferon Beta Production by Targeting RIG-I and MDA5. J Virol 91:e02396-16.

30. Qin Y, Xue B, Liu C, Wang X, Tian R, Xie Q, Guo M, Li G, Yang D, Zhu H. 2017. NLRXi Mediates MAVS Degradation To Attenuate the Hepatitis C Virus-Induced Innate Immune Response through PCBP2. J Virol 91:e01264-17.

31. Medina GN, Segundo FD-S, Stenfeldt C, Arzt J, de los Santos T. 2018. The Different Tactics of Foot-andMouth Disease Virus to Evade Innate Immunity. Front Microbiol 12:e2644

32. Wang D, Fang L, Li K, Zhong H, Fan J, Ouyang C, Zhang H, Duan E, Luo R, Zhang Z, Liu X, Chen H, Xiao S. 2012. Foot-and-mouth disease virus $3 \mathrm{C}$ protease cleaves NEMO to impair innate immune signaling. J Viro 86:9311-9322

33. Guarné A, Tormo J, Kirchweger R, Pfistermueller D, Fita I, Skern T. 1998. Structure of the foot-and-mouth disease virus leader protease: A papain-like fold adapted for self-processing and eIF4G recognition. EMBO 17:7469-7479.

34. Devaney MA, Vakharia VN, Lloyd RE, Ehrenfeld E, Grubman MJ. 1988. Leader protein of foot-and-mouth disease virus is required for cleavage of the p220 component of the cap-binding protein complex. J Viro 62:4407-9.

35. de Los Santos T, Diaz-San Segundo F, Grubman MJ. 2007. Degradation of nuclear factor kappa B during foot-and-mouth disease virus infection. J Virol 81:12803-12815. 
36. de los Santos T, Diaz-San Segundo F, Zhu J, Koster M, Dias CCA, Grubman MJ. 2008. A Conserved Domain in the Leader Proteinase of Foot-and-Mouth Disease Virus Is Required for Proper Subcellular Localization and Function. J Virol 83:1800-10.

37. Wang D, Fang L, Luo R, Ye R, Fang Y, Xie L, Chen H, Xiao S. 2010. Foot-and-mouth disease virus leader proteinase inhibits dsRNA-induced type I interferon transcription by decreasing interferon regulatory factor 3/7 in protein levels. Biochem Biophys Res Commun 399:72-78.

38. Medina GN, Knudsen GM, Greninger AL, Kloc A, Díaz-San Segundo F, Rieder E, Grubman MJ, DeRisi JL, de los Santos T. 2017. Interaction between FMDV Lpro and transcription factor ADNP is required for optimal viral replication. Virology 505:12-22.

39. Wang D, Fang L, Li P, Sun L, Fan J, Zhang Q, Luo R, Liu X, Li K, Chen H, Chen Z, Xiao S. 2011. The Leader Proteinase of Foot-and-Mouth Disease Virus Negatively Regulates the Type I Interferon Pathway by Acting as a Viral Deubiquitinase. J Virol 85:3758-3766.

40. Swatek KN, Aumayr M, Pruneda JN, Visser LJ, Berryman S, Kueck AF, Geurink PP, Ovaa H, van Kuppeveld FJM, Tuthill TJ, Skern T, Komander D. 2018. Irreversible inactivation of ISG15 by a viral leader protease enables alternative infection detection strategies. Proc Natl Acad Sci U S A 115:2371-2376.

41. Steinberger J, Skern T. 2014. The leader proteinase of foot-and-mouth disease virus : structure-function relationships in a proteolytic virulence factor. Biol Chem 395:1179-1185.

42. Swatek KN, Usher JL, Kueck AF, Gladkova C, Mevissen TET, Pruneda JN, Skern T, Komander D. 2019. Insights into ubiquitin chain architecture using Ub-clipping. Nature 572:533-7.

43. Hato S V, Ricour C, Schulte BM, Lanke KHW, de Bruijn M, Zoll J, Melchers WJG, Michiels T, van Kuppeveld FJM. 2007. The mengovirus leader protein blocks interferon-alpha/beta gene transcription and inhibits activation of interferon regulatory factor 3. Cell Microbiol 9:2921-2930.

44. Langereis MA, Feng $Q$ van Kuppeveld FJ. 2013. MDA5 localizes to stress granules, but this localization is not required for the induction of type I interferon. J Virol 87:6314-25.

45. Visser LJ, Medina GN, Rabouw HH, de Groot RJ, Langereis MA, de Los Santos T, van Kuppeveld FJM. 2019. Foot-and-Mouth Disease Virus Leader Protease Cleaves $\mathrm{G}_{3} \mathrm{BP}_{1}$ and $\mathrm{G}_{3} \mathrm{BP} 2$ and Inhibits Stress Granule Formation. J Virol 93:e00922-18.

46. Roberts PJ, Belsham GJ. 1995. Identification of critical amino acids within the foot-and-mouth disease virus Leader protein, a cysteine protease. Virology 213:140-6.

47. de Los Santos T, de Avila Botton S, Weiblen R, Grubman MJ. 2006. The leader proteinase of foot-and-mouth disease virus inhibits the induction of beta interferon mRNA and blocks the host innate immune response. I Virol 80:1906-14.

48. Aumayr M, Fedosyuk S, Ruzicska K, Sousa-Blin C, Kontaxis G, Skern T. 2015. NMR analysis of the interaction of picornaviral proteinases $\mathrm{Lb}$ and $2 \mathrm{~A}$ with their substrate eukaryotic initiation factor $4 \mathrm{GII}$. Protein Sci 24:1979-1996.

49. Mayer C, Neubauer D, Nchinda a. T, Cencic R, Trompf K, Skern T. 2008. Residue Li43 of the Foot-and-Mouth Disease Virus Leader Proteinase Is a Determinant of Cleavage Specificity. J Virol 82:4656-4659.

50. Goncalves A, Bürckstümmer T, Dixit E, Scheicher R, Górna MW, Karayel E, Sugar C, Stukalov A, Berg T, Kralovics R, Planyavsky M, Bennett KL, Colinge J, Superti-Furga G. 2011. Functional dissection of the TBK1 molecular network. PLoS One 6:e23971.

51. Rodríguez Pulido M, Sánchez-Aparicio MT, Martínez-Salas E, García-Sastre A, Sobrino F, Sáiz M. 2018. Innate immune sensor LGP2 is cleaved by the Leader protease of foot-and-mouth disease virus. PLoS Pathog
14:e1007135.

52. Pilli M, Arko-Mensah J, Ponpuak M, Roberts E, Master S, Mandell MA, Dupont N, Ornatowski W, Jiang S, Bradfute SB, Bruun JA, Hansen TE, Johansen T, Deretic V. 2012. TBK-1 Promotes Autophagy-Mediated Antimicrobial Defense by Controlling Autophagosome Maturation. Immunity 37:223-34.

53. Ahmad L, Mashbat B, Leung C, Brookes C, Hamad S, Krokowski S, Shenoy AR, Lorenzo L, Levin M, O'Hare P, Zhano SY, Casanova JL, Mostowy S, Sancho-Shimizu V. 2019. Human TANK-binding kinase 1 is required for early autophagy induction upon herpes simplex virus I infection. J Allergy Clin Immunol 143:765-769.e7.
54. Herhaus L, Bhaskara RM, Lystad AH, Gestal-Mato U, Covarrubias-Pinto A, Bonn F, Simonsen A, Hummer G, Dikic I. 2020. TBK1-mediated phosphorylation of LC3C and GABARAP-L2 controls autophagosome shedding by ATG4 protease. EMBO Rep 21:e48317.

55. Jackson WT, Giddings TH, Taylor MP, Mulinyawe S, Rabinovitch M, Kopito RR, Kirkegaard K. 2005. Subversion of Cellular Autophagosomal Machinery by RNA Viruses. PLoS Biol 3:e156.

56. Schlegel A, Giddings TH, Ladinsky MS, Kirkegaard K. 1996. Cellular origin and ultrastructure of membranes induced during poliovirus infection. J Virol 70:6576-88.

57. Hsu N-Y, Ilnytska O, Belov G, Santiana M, Chen Y-H, Takvorian PM, Pau C, van der Schaar H, Kaushik-Basu N, Balla T, Cameron CE, Ehrenfeld E, van Kuppeveld FIM, Altan-Bonnet N. 2010. Viral Reorganization of the Secretory Pathway Generates Distinct Organelles for RNA Replication. Cell 141:799-811.

58. van der Grein SG, Defourny KAY, Rabouw HH, Galiveti CR, Langereis MA, Wauben MHM, Arkesteijn GJA van Kuppeveld FJM, Nolte-'t Hoen ENM. 2019. Picornavirus infection induces temporal release of multiple extracellular vesicle subsets that differ in molecular composition and infectious potential. PLoS Pathog 15:1007594.

59. Bird SW, Maynard ND, Covert MW, Kirkegaard K. 2014. Nonlytic viral spread enhanced by autophagy components. Proc Natl Acad Sci U S A 111:13081-6.

60. O'Donnell V, Pacheco JM, LaRocco M, Burrage T, Jackson W, Rodriguez LL, Borca M V., Baxt B. 2011. Footand-mouth disease virus utilizes an autophagic pathway during viral replication. Virology 410:142-50.

61. Gladue DP, O’Donnell V, Baker-Branstetter R, Holinka LG, Pacheco JM, Fernandez-Sainz I, Lu Z, Brocchi E, Baxt B, Piccone ME, Rodriguez L, Borca M V. 2012. Foot-and-Mouth Disease Virus Nonstructural Protein 2C Interacts with Beclini, Modulating Virus Replication. J Virol 86:12080-90.

62. Dixit E, Boulant S, Zhang Y, Lee ASY, Odendall C, Shum B, Hacohen N, Chen ZJ, Whelan SP, Fransen M, Nibert ML, Superti-Furga G, Kagan JC. 2010. Peroxisomes Are Signaling Platforms for Antiviral Innate Immunity. Cell 141:668-81.

63. Horner SM, Liu HM, Park HS, Briley J, Gale M. 2011. Mitochondrial-associated endoplasmic reticulum membranes (MAM) form innate immune synapses and are targeted by hepatitis $C$ virus. Proc Natl Acad Sci U S A 108:14590-5.

64. Medina GN, Azzinaro P, Ramirez-Medina E, Gutkoska J, Fang Y, Diaz-San Segundo F, de los Santos T. 2020. Impairment of the deISGylation activity of FMDV Lpro causes attenuation in vitro and in vivo. J Virol ePub ahead of print.

65. Lindner HA, Fotouhi-Ardakani N, Lytvyn V, Lachance P, Sulea T, Ménard R. 2005. The Papain-Like Protease from the Severe Acute Respiratory Syndrome Coronavirus Is a Deubiquitinating Enzyme. J Virol 79:1519915208 .

66. Ratia K, Saikatendu KS, Santarsiero BD, Barreto N, Baker SC, Stevens RC, Mesecar AD. 2006. Severe acute respiratory syndrome coronavirus papain-like-protease: Structure of a viral deubiquitinating enzyme. Proc Natl Acad Sci U S A 103:5717-22.

67. Lindner HA, Lytvyn V, Qi H, Lachance P, Ziomek E, Ménard R. 2007. Selectivity in ISG15 and ubiquitin recognition by the SARS coronavirus papain-like protease. Arch Biochem Biophys 466:8-14.

68. Wojdyla JA, Manolaridis I, van Kasteren PB, Kikkert M, Snijder EJ, Gorbalenya AE, Tucker PA. 2010. Papain Like Protease I from Transmissible Gastroenteritis Virus: Crystal Structure and Enzymatic Activity toward Viral and Cellular Substrates. J Virol 84:10063-73.

69. Chen Z, Wang Y, Ratia K, Mesecar AD, Wilkinson KD, Baker SC. 2007. Proteolytic Processing and Deubiquitinating Activity of Papain-Like Proteases of Human Coronavirus NL63. J Virol 81:6007-6018.

70. Mielech AM, Kilianski A, Baez-Santos YM, Mesecar AD, Baker SC. 2014. MERS-CoV papain-like protease has deISGylating and deubiquitinating activities. Virology 450-451:64-70.

71. Van Kasteren PB, Bailey-Elkin BA, James TW, Ninaber DK, Beugeling C, Khajehpour M, Snijder EJ, Mark BL, Kikkert M. 2013. Deubiquitinase function of arterivirus papain-like protease 2 suppresses the innate immune response in infected host cells. Proc Natl Acad Sci U S A 110:E838-47. 
72. van Kasteren PB, Knaap RCM, van den Elzen P, Snijder EJ, Balasuriya UBR, van den Born E, Kikkert M. 2015. In vivo assessment of equine arteritis virus vaccine improvement by disabling the deubiquitinase activity of papain-like protease 2. Vet Microbiol 178:132-7.

73. Bailey-Elkin BA, Knaap RCM, Johnson GG, Dalebout TJ, Ninaber DK, van Kasteren PB, Bredenbeek PJ, Snijder EJ, Kikkert M, Mark BL. 2014. Crystal structure of the Middle East respiratory syndrome coronavirus (MERS-CoV) papain-like protease bound to ubiquitin facilitates targeted disruption of deubiquitinating activity to demonstrate its role in innate immune suppression. J Biol Chem 289:34667-82.

74. Haghighat A, Svitkin Y, Novoa I, Kuechler E, Skern T, Sonenberg N. 1996. The eIF4G-eIF4E complex is the target for direct cleavage by the rhinovirus $2 \mathrm{~A}$ proteinase. J Virol.

75. Ventoso I, MacMillan SE, Hershey JW b., Carrasco L. 1998. Poliovirus 2A proteinase cleaves directly the eIF4G subunit of eIF-4F complex. FEBS Lett 435:79-83.

76. Wang B, Xi X, Lei X, Zhang X, Cui S, Wang J, Jin Q Zhao Z. 2013. Enterovirus 71 Protease 2Apro Targets MAVS to Inhibit Anti-Viral Type I Interferon Responses. PLoS Pathog 9:e1003231.

77. Visser LJ, Langereis MA, Rabouw HH, Wahedi M, Muntjewerff EM, de Groot RJ, van Kuppeveld FJM. 2019. Essential Role of Enterovirus 2A Protease in Counteracting Stress Granule Formation and the Induction of Type I Interferon. J Virol 93.

78. Yang $\mathrm{X}, \mathrm{Hu} \mathrm{Z}$, Fan S, Zhang $\mathrm{Q}$ Zhong $\mathrm{Y}$, Guo D, Qin $\mathrm{Y}$, Chen M. 2018. Picornavirus $2 \mathrm{~A}$ protease regulates stress granule formation to facilitate viral translation. PLOS Pathog 14:1006901.

79. Visser LJ, Medina GN, Rabouw HH, de Groot RJ, Langereis MA, de Los Santos T, van Kuppeveld FJM. 2018. FMDV leader protease cleaves $\mathrm{G}_{3} \mathrm{BP}_{1}$ and $\mathrm{G}_{3} \mathrm{BP} 2$ and inhibits stress granule formation. J Virol.

80. Redondo N, Sanz MA, Welnowska E, Carrasco L. 2011. Translation without eIF2 Promoted by Poliovirus 2A Protease. PLoS One 6:e25699.

81. Moral-López P, Alvarez E, Redondo N, Skern T, Carrasco L. 2014. L protease from foot and mouth disease virus confers eIF2-independent translation for mRNAs bearing picornavirus IRES. FEBS Lett 588:40534059.

82. Morales DJ, Monte K, Sun L, Struckhoff JJ, Agapov E, Holtzman MJ, Stappenbeck TS, Lenschow DJ. 2015. Novel Mode of ISG15-Mediated Protection against Influenza A Virus and Sendai Virus in Mice. J Virol 89:337349.

83. Nakashima H, Nguyen T, Goins WF, Chiocca EA. 2015. Interferon-stimulated gene 15 (ISG15) and ISG15linked proteins can associate with members of the selective autophagic process, histone deacetylase 6 (HDAC6) and SQSTM1/p62. J Biol Chem 290:1485-95.

84. Xu D, Zhang T, Xiao J, Zhu K, Wei R, Wu Z, Meng H, Li Y, Yuan J. 2015. Modification of BECN1 by ISG15 plays a crucial role in autophagy regulation by type I IFN/interferon. Autophagy 11:617-628.

85. Baldanta S, Fernández-Escobar M, Acín-Perez R, Albert M, Camafeita E, Jorge I, Vázquez J, Enríquez JA, Guerra S. 2017. ISG15 governs mitochondrial function in macrophages following vaccinia virus infection. PLOS Pathog 13:e1006651.

86. LaRocco M, Krug PW, Kramer E, Ahmed Z, Pacheco JM, Duque H, Baxt B, Rodriguez LL. 2013. A continuous bovine kidney cell line constitutively expressing bovine $\alpha V \beta 6$ integrin has increased susceptibility to footand-mouth disease virus. J Clin Microbiol 51:1714-1720.

87. Langereis MA, Rabouw HH, Holwerda M, Visser LJ, van Kuppeveld FJM. 2015. Knockout of cGAS and STING Rescues Virus Infection of Plasmid DNA-Transfected Cells. J Virol 89:11169-73.

88. Rieder E, Bunch T, Brown F, Mason PW. 1993. Genetically Engineered Foot-and-Mouth Disease Viruses with Poly(C) Tracts of Two Nucleotides Are Virulent in Mice. J Virol 67:5139-5145.

89. Piccone ME, Rieder E, Mason PW, Grubman MJ. 1995. The foot-and-mouth disease virus leader proteinase gene is not required for viral replication. J Virol 69:5376-5382.

90. Van Der Schaar HM, Leyssen P, Thibaut HJ, De Palma A, Van Der Linden L, Lanke KHW, Lacroix C, Verbeken E, Conrath K, MacLeod AM, Mitchell DR, Palmer NJ, Van De Poel H, Andrews M, Neyts J, Van Kuppeveld FM. 2013 A Novel, Phosphatidylinositol 4-Kinase III-beta. Antimicrob Agents Chemother 57:4971-4981.
91. Lin R, Heylbroeck C, Pitha PM, Hiscott J. 1998. Virus-Dependent Phosphorylation of the IRF-3 Transcription Factor Regulates Nuclear Translocation, Transactivation Potential, and Proteasome-Mediated Degradation. Mol Cell Biol 18:2986-96.

92. Rabouw HH, Langereis MA, Knaap RCM, Dalebout TJ, Canton J, Sola I, Enjuanes L, Bredenbeek PJ, Kikker M, de Groot RJ, van Kuppeveld FJM. 2016. Middle East Respiratory Coronavirus Accessory Protein 4a Inhibit PKR-Mediated Antiviral Stress Responses. PLoS Pathog 12:e1005982.

93. Sharma S, TenOever BR, Grandvaux N, Zhou GP, Lin R, Hiscott J. 2003. Triggering the interferon antiviral response through an IKK-related pathway. Science (80- ) 300:1148-51.

94. Yoneyama M, Kikuchi M, Matsumoto K, Imaizumi T, Miyagishi M, Taira K, Foy E, Loo Y-M, Gale M, Akira S, Yonehara S, Kato A, Fujita T. 2005. Shared and unique functions of the DExD/H-box helicases RIG-I, MDA5, and LGP2 in antiviral innate immunity. J Immunol 175:2851-8.

95. Kirchweger R, Ziegler E, Lamphear BJ, Waters D, Liebig - HD, Sommergruber W, Sobrino F, Hohenadl C, Blaas D, Rhoads RE, Skern T. 1994. Foot-and-mouth disease virus leader proteinase: Purification of the L form and determination of its cleavage site on eIF-4 $\gamma$. J Virol 68:5677-84.

96. Geurink PP, El Oualid F, Jonker A, Hameed DS, Ovaa H. 2012. A General Chemical Ligation Approach Towards Isopeptide-Linked Ubiquitin and Ubiquitin-Like Assay Reagents. ChemBioChem 13:293-7.

97. Mevissen TET, Kulathu Y, Mulder MPC, Geurink PP, Maslen SL, Gersch M, Elliott PR, Burke JE, van Tol BDM, Akutsu M, El Oualid F, Kawasaki M, Freund SMV, Ovaa H, Komander D. 2016. Molecular basis of Lys1polyubiquitin specificity in the deubiquitinase Cezanne. Nature 538:402-405. 


\section{Supplementary Information}
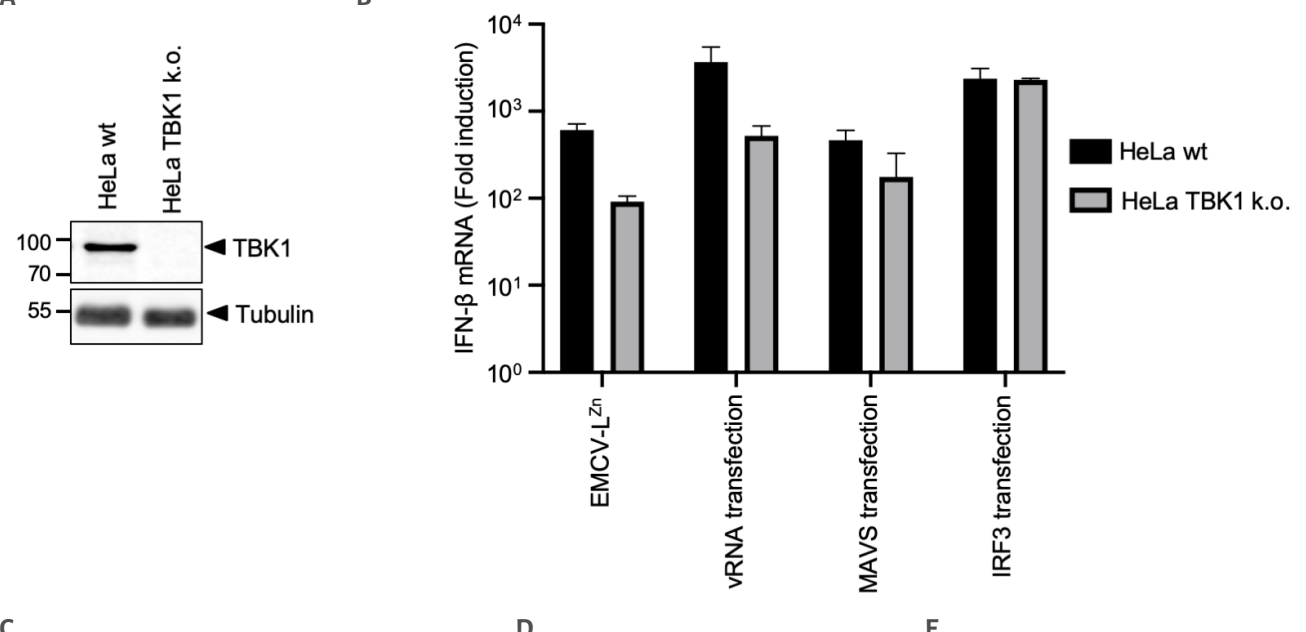

E
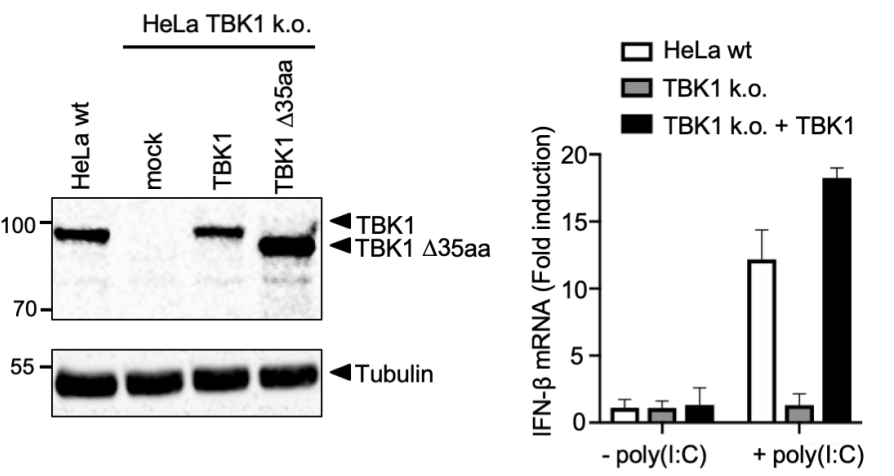

TBK1 k.o. + TBK1

口TBK1 k.o. + TBK1 $\triangle 35 \mathrm{aa}$

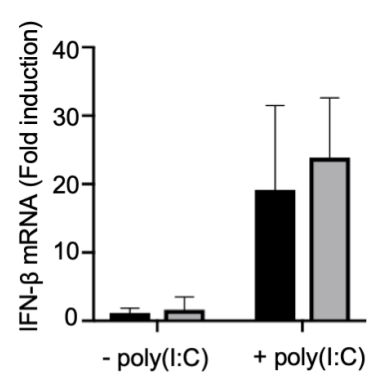

Figure S1: N-terminal TBK1 cleavage fragment can facilitate RLR signaling. (A) HeLa R19 TBK1 K.o. cells were generated using CRISPR/cas9 technology. Wt and TBK1 k.o. cells were lysed and lysates subjected to Western Blot analysis for TBK1 and tubulin. (B) IFN- $\beta$ induction upon various triggers of RLR signaling was compared in wt and TBK1 k.o. cells. Cells

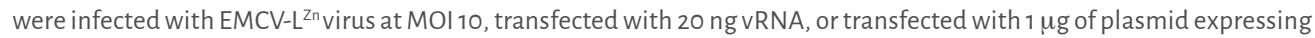
MAVS or IRF3. Cells were lysed at $8 \mathrm{~h}$ pi or transfection. Total RNA was isolated and used for RT-qPCR analysis for IFN- $\beta$ and actin mRNA. The IFN- $\beta$ levels are depicted as a fold induction compared to levels in mock-treated cells, after correction for actin mRNA levels. Error bars depict the SD. (C) HeLa R19 TBK1 K.o. cells were transfected with $2 \mu \mathrm{g}$ plasmid expressing full-length or truncated TBK1 (TBK1 $\triangle 35$ aa). TBK1 $\triangle 35$ aa is representative for the Lro-generated $\mathrm{N}$-terminal $^{\text {pro }}$ cleavage product. Cells were lysed and lysates subjected to Western Blot analysis for TBK1 and tubulin. (D) TBK1 K.o. cells were reconstituted with full-length TBK1 as described for (C) and subsequently transfected with $100 \mathrm{ng}$ poly(l:C). Cells were lysed at $8 \mathrm{~h}$ post transfection of poly(I:C). Total RNA was isolated and used for RT-qPCR analysis for IFN- $\beta$ and actin mRNA. The IFN- $\beta$ levels are depicted as a fold induction compared to levels in mock-treated cells, after correction for actin mRNA levels. Error bars depict the SD. (E) TBK1 k.o. cells were reconstituted with full-length or truncated TBK1 (TBK1 $\triangle 35$ aa) as described for (C). Subsequent steps as described for (D). Error bars depict the SD. 


\section{Chapter 5}

Essential role of enterovirus $2 \mathrm{~A}$ protease in counteracting stress granule formation and the induction of type I interferon

Linda J. Visser ${ }^{1}$, Martijn A. Langereis ${ }^{1}$, Huib H. Rabouw ${ }^{1}$, Maryam Wahedi ${ }^{1}$, Elke M. Muntjewerff, Raoul J. de Groot ${ }^{1}$, Frank J.M. van Kuppeveld ${ }^{1 *}$

${ }^{1}$ Virology Division, Department of Infectious Diseases and Immunology, Faculty of Veterinary Medicine, Utrecht University, The Netherlands

"Corresponding author. 


\section{Abstract}

Most viruses have acquired mechanisms to suppress antiviral IFN- $\alpha / \beta$ and stress responses. Enteroviruses actively counteract the induction of IFN- $\alpha / \beta$ gene transcription and stress granule (SG) formation, which are increasingly implicated as a platform for antiviral signaling, but the underlying mechanisms remain poorly understood. Both viral proteases $\left(2 \mathrm{~A}^{\text {pro }}\right.$ and $3 \mathrm{C}^{\text {pro }}$ ) have been implicated to suppress these responses, but these conclusions predominantly rely on ectopic overexpression of viral proteases or addition of purified viral proteases to cell lysates. Here, we present a detailed and comprehensive comparison of the effect of individual enterovirus proteases on the formation of SGs and the induction of IFN- $\alpha / \beta$ gene expression in infected cells, for representative members of the enterovirus species EV-A to EV-D. First, we show that SG formation and IFN- $\beta$ induction are suppressed in cells infected with EV-A71, CV-B3, CV-A21 and EV-D68. By introducing genes encoding CV-B3 proteases in a recombinant encephalomyocarditis virus (EMCV) that was designed to efficiently activate antiviral responses, we show that $\mathrm{CV}-\mathrm{B} 32 \mathrm{~A}^{\text {pro }}$, but not $3 \mathrm{C}^{\text {pro }}$, is the major antagonist that counters $S G$ formation and IFN- $\beta$ gene transcription, and that $2 \mathrm{~A}^{\text {pro's }}$ proteolytic activity is essential for both functions. $2 \mathrm{~A}^{\text {pro }}$ efficiently suppressed SG formation despite PKR activation and eIF2 $\alpha$ phosphorylation, suggesting that $2 \mathrm{~A}^{\text {pro }}$ antagonizes $\mathrm{SG}$ assembly or promotes their disassembly. Finally, we show that the ability to suppress SG formation and IFN- $\beta$ gene transcription is conserved in the $2 \mathrm{~A}^{\text {pro }}$ of EV-A71, CV-A21 and EV-D68. Collectively, our results indicate that enterovirus $2 \mathrm{~A}^{\text {pro }}$ plays a key role in inhibiting innate antiviral cellular responses.

\section{Importance}

Enteroviruses are important pathogens that can cause a variety of diseases in humans, including aseptic meningitis, myocarditis, hand-foot-and-mouth disease, conjunctivitis and acute flaccid paralysis. Like many other viruses, enteroviruses must counteract antiviral cellular responses to establish an infection. It has been suggested that enterovirus proteases cleave cellular factors to perturb antiviral pathways, but the exact contribution of viral proteases $2 \mathrm{~A}^{\text {pro }}$ and $3 \mathrm{C}^{\text {pro }}$ remains elusive. Here, we show that $2 \mathrm{~A}^{\text {pro, }}$, but not $3 \mathrm{C}^{\text {pro }}$, of all four human EV species (EV-A to EV-D) inhibits SG formation and IFN- $\beta$ gene transcription. Our observations suggest that enterovirus $2 \mathrm{~A}^{\text {pro }}$ has a conserved function in counteracting antiviral host responses, and thereby is the main enterovirus "security protein". Understanding the molecular mechanisms of enterovirus immune evasion strategies may help to develop countermeasures to control infections with these viruses.

\section{Introduction}

Picornaviruses are a large and diverse family of small $(\sim 30 \mathrm{~nm})$ non-enveloped viruses with a positive-sense RNA genome. Especially the genus Enterovirus encompasses many human pathogens with implications for public health. The enteroviruses are categorized into fifteen enterovirus species (EV-A to EV-L) and three rhinovirus species (RV-A to RVC). Human pathogens are found amongst the EV-A to EV-D and the three rhinovirus species. The best-known pathogenic enterovirus is poliovirus (PV), the causative agent of poliomyelitis. In addition, coxsackie A and B viruses, echoviruses and several numbered enteroviruses (e.g. EV-A71 and EV-D68) cause a broad range of diseases, such as aseptic meningitis, myocarditis, hand-foot-and-mouth disease, conjunctivitis and acute flaccid paralysis. Rhinoviruses are the leading cause of the common cold, and are associated with exacerbations of asthma and chronic obstructive pulmonary disease (COPD) (reviewed in (1)). The enterovirus genome is translated into one polyprotein that is autocatalytically processed into the structural proteins $\left(\mathrm{VP}_{1}, \mathrm{VP}_{2}, \mathrm{VP}_{3}, \mathrm{VP} 4\right)$, the non-structural proteins $(2 \mathrm{~A}, 2 \mathrm{~B}, 2 \mathrm{C}, 3 \mathrm{~A}, 3 \mathrm{~B}, 3 \mathrm{C}, 3 \mathrm{D}$ ) and several cleavage intermediates (e.g. 3CD) (reviewed in (1)). While the structural proteins make up the viral capsid, the non-structural proteins are involved in virus replication and/or interfere with antiviral responses. Non-structural proteins $2 \mathrm{~A}$ and $3 \mathrm{C}$ are the proteases involved in processing of the viral polyprotein. While $3 \mathrm{C}^{\text {pro }}$ is responsible for the majority of the cleavages needed for polyprotein processing, $2 \mathrm{~A}^{\text {pro }}$ cleaves at the junction between the structural proteins ( $\mathrm{P} 1$ region) and its own $\mathrm{N}$-terminus (reviewed in (1)). Besides their essential role in polyprotein processing, both proteases also cleave host cell factors to optimize virus replication. For example, $2 \mathrm{~A}^{\text {pro }}$ mediates host shutoff by cleaving translation initiation factor $4 \mathrm{G}(\mathrm{eIF} 4 \mathrm{G})(2)$.

In response to virus infections, cells activate several antiviral responses. A well-known antiviral response is the induction of type I interferons (IFN- $\alpha / \beta)$. Viral genome replication generates double-stranded RNA(dsRNA) replication intermediates, which can be recognized by cytoplasmic RIG-I like receptors (RLRs). Enterovirus replication intermediates are predominantly recognized by MDA5(3-5). However, recent evidence suggests that RIG-I may also contribute to the induction of IFN- $\alpha / \beta$ in certain cell types (6). Upon recognition of viral dsRNA, MDA5 interacts with MAVS, which subsequently activates TRAF3, and TBK1. TBK1 phosphorylates the transcription factors IRF3 and IRF7, resulting in their activation and dimerization. Simultaneously, TRAF3 activates the transcription factor NFKB. Upon activation, IRF3, IRF7 and NFKB localize to the nucleus, where they induce expression of IFN- $\alpha / \beta$ and other proinflammatory cytokines. Subsequent IFN- $\alpha / \beta$ signaling via the type I IFN receptor (IFNAR) and the JAK-STAT pathway induces the expression of hundreds of interferon stimulated genes (ISGs). IFN- $\alpha / \beta$ signals in autocrine and paracrine ways to induce a tissue-wide antiviral state, thereby limiting viral spread (reviewed in $(7,8))$. 
One of the ISGs is dsRNA-dependent protein kinase R (PKR), a sensor protein of the Integrated Stress Response (ISR). When PKR binds dsRNA ligands, it dimerizes, autophosphorylates, and subsequently phosphorylates the $\alpha$-subunit of translation initiation factor 2 (eIF2 $\alpha$ ). Phosphorylated eIF2 $\alpha$ (p-eIF2 $\alpha$ ) represses translation and thereby impairs virus propagation. Stalled messenger ribonucleoproteins (mRNPs) consisting of mRNA, small ribosomal subunits, translation initiation factors (eIFs), and several auxiliary proteins - are stored in cytoplasmic aggregates known as stress granules (SG). SG formation is directed by SG scaffolding proteins, such as GTPase-activating protein-binding proteins 1 and 2 (G3BP1 and G3BP2) and $\mathrm{T}$ cell-restricted intracellular antigen I (TIA-1) (reviewed in (9)). Rather than merely being an outcome of translation inhibition, SGs have also been suggested to serve as a platform for antiviral signaling. Multiple signaling molecules - including MDA5, RIG-I, PKR and TRAF2 - are recruited to SGs and this localization has been suggested to regulate their activity (10-16). Further evidence for an antiviral function of SGs is the growing evidence that many viruses apart from their effect on the upstream signaling cascade - have evolved mechanisms to counteract SG formation by targeting SG components $(15,17-20)$. The exact function of SGs, however, remains to be established.

To establish a productive infection, enteroviruses actively suppress antiviral responses. Several studies have shown that enteroviruses suppress IRF3 dimerization and thereby IFN- $\alpha / \beta$ gene transcription $(11,21-25)$. Both viral proteases, $2 \mathrm{~A}^{\text {pro }}$ and $3 \mathrm{C}^{\text {pro }}$, cleave various factors of the RLR signaling pathway, implicating them in the suppression of IFN- $\alpha / \beta$ gene transcription. Overexpression of $3 \mathrm{C}^{\text {pro }}$ of several enteroviruses has been shown to result in the cleavage of MAVS, IRF7 and IRF9 and - by consequence - in the suppression of a coexpressed IFN reporter construct (24-28). However, in several of these studies, convincing evidence that the cleavage products observed upon $3 \mathrm{C}^{\text {pro }}$ overexpression are the same as in enterovirus-infected cells is lacking. In fact, we and others have identified $2 \mathrm{~A}^{\text {pro }}$ as the viral protease responsible for cleaving $\operatorname{MAVS}(21,22)$. Addition of recombinant $2 \mathrm{~A}^{\text {pro }}$, but not $3 \mathrm{C}^{\text {pro }}$, to cell lysates resulted in the appearance of MAVS cleavage products of the same size as observed in infected cells. These cleavage products were also observed when $2 \mathrm{~A}^{\text {pro }}$, but not $3 \mathrm{C}^{\text {pro }}$, was expressed by a recombinant $\mathrm{EMCV}$, a picornavirus that by itself does not cleave components of the RLR pathway (21). Besides cleaving MAVS, $2 \mathrm{~A}^{\text {pro }}$ has also been suggested to cleave MDA5 (21), which contradicted earlier suggestions that MDA5 is degraded by the proteasome (29). Meanwhile, $3 C^{\text {pro }}$ cleaves RIG-I $(21,30)$. Contradictory results have also been reported on the mechanism how enteroviruses interfere with IFN- $\alpha / \beta$ signaling. Some studies reported that EV-A71 infection reduces the expression levels of the IFN receptor, blocks STATi and STAT2 phosphorylation, and suppresses expression of multiple ISGs, through the action of $2 \mathrm{~A}^{\text {pro }}(31,32)$. In another study, however, it was suggested that EV-A71 infection does not affect STATr and STAT2 phosphorylation, but that $3 \mathrm{C}^{\text {pro }}$ prevents these molecules from entering the nucleus (33). Overall, these conflicting data indicate that the physiological role of the $2 \mathrm{~A}^{\text {pro }}$ - and/or $3 \mathrm{C}^{\text {pro- }}$-mediated cleavages of signaling molecules for the suppression of the RLR and JAK-STAT pathways requires further investigation.

Enteroviruses also actively suppress the ISR. Infection of cells with PV, CV-B3 and EV-A71 results in the formation of small SGs early in infection, but these disappear later in infection (34-37). Again, conflicting observations about the identity of the viral antagonist have been reported. Initially, the suppression of SGs was attributed to $3 C^{\text {pro }}(34-36) \cdot 3 C^{\text {pro }}$ was shown to cleave G3BPI and suppress SG formation, and overexpression of a cleavage-resistant G3BP1 decreased virus replication, although this decrease was rather modest $(34,36,38)$. Recently, however, evidence was presented that $2 \mathrm{~A}^{\text {pro }}$ from EV-A71, PV or CV suppresses SG formation induced by infection, as well as upon induction by sodium arsenite or heat shock (37).

In this study, we set out to directly compare the abilities of $2 \mathrm{~A}^{\text {pro }}$ and $3 \mathrm{C}^{\text {pro }}$ of multiple enteroviruses to suppress IFN- $\alpha / \beta$ induction and SG formation, in infected cells. As described above, we previously introduced the genes encoding $2 \mathrm{~A}^{\text {pro }}$ and $3 \mathrm{C}^{\text {pro }}$ of several enteroviruses in EMCV to study $2 \mathrm{~A}^{\text {pro }}$ - and $3 \mathrm{C}^{\text {pro }}$-mediated cleavages in infected cells (21). However, these recombinant EMCVs contained an intact antagonist of the antiviral responses, i.e. the leader (L) protein, and therefore these viruses could not be used to study the effect of the enterovirus proteases on IFN- $\alpha / \beta$ mRNA levels and SG formation. To overcome this, we introduced the genes encoding the enterovirus proteases in a mutant EMCV containing mutations that inactivate the leader protein (EMCV-L $\left.\mathrm{L}^{2 n}\right)(3,11,39)$. Using these recombinant viruses, we provide a comprehensive and detailed analysis of the effect of the proteases of representative members of the enterovirus species EV-A to EV-D on the formation of SGs and the induction of IFN- $\alpha / \beta$. We demonstrate that $2 \mathrm{~A}^{\text {pro }}$, not $3 \mathrm{C}^{\text {pro }}$, of $E V-$ A71, CV-B3, CV-A21 and EV-D68 strongly suppresses IFN- $\alpha / \beta$ induction and SG formation in virus-infected cells, thereby pinpointing $2 \mathrm{~A}^{\mathrm{pro}}$ as a major antagonist of these antiviral responses.

\section{Results}

Multiple enteroviruses suppress SG formation. We first determined whether representative members of the enterovirus species EV-A - EV-D share the ability to suppress SGs. To this end, we infected HeLa cells with EV-A71, CV-B3, CV-A21 or EV-D68 and visualized SGs. We also infected cells with EMCV-L $\mathrm{L}^{\text {zn }}$, which induces SG formation (11) and therefore served as a positive control. At $4 \mathrm{~h}$ and $6 \mathrm{~h}$ post infection (hpi) cells were fixed and we performed immunofluorescence staining for dsRNA, to visualize viral replication, and the SG markers 
G3BP1 and eIF3. Infection with all the viruses led to an increase in dsRNA signal, indicating that all viruses replicated efficiently. In the cells infected with the different enteroviruses we observed small SGs at $4 \mathrm{hpi}$ (Fig 1). These SGs were no longer detected at $6 \mathrm{hpi}$ in cells infected with CV-B3 or CV-A21, while the majority of the cells infected with EV-A71 and EVD68 also lacked SGs at 6 hpi (Fig 1). Suppression of SG formation was previously reported for PV (34), CV-B3 (35) and - while this work was in progress - also for EV-A71 $(36,37)$. Here, we provide evidence that also CV-A21, another member of the EV-C species, and EV-D68, an EV-D species member, suppress the formation of SGs. Taken together, our data suggest that the ability to counteract SG formation is conserved amongst all human enterovirus species (i.e. EV-A to EV-D).

Overexpression of enterovirus $2 \mathrm{~A}^{\text {pro }}$ suppresses sodium arsenite-induced SGs. To better understand the effects of enterovirus proteases on $S G$ formation, we overexpressed $2 \mathrm{~A}^{\text {pro }}$ and $3 \mathrm{C}^{\text {pro }}$ from EV-A71, CV-B3 or PV as EGFP fusion proteins and visualized SGs in the transfected cells. The cells were treated with sodium arsenite to induce SG formation. Afterwards we performed immunofluorescence staining against G3BP1 and TIA-1 (Fig. 2). In our hands, enterovirus $2 \mathrm{~A}^{\text {pro }}$ inhibited $S G$ formation (Fig. $2 \mathrm{~A}$ ). In cells expressing EGFP-2A ${ }^{\text {pro }}$ we did not detect any G3BP1 positive SGs. However, we did observe a few TIA-1 granules. Meanwhile, expression of EGFP-3C $\mathrm{C}^{\text {pro }}$ had no effect on SG formation (Fig. 2B). These findings suggest that enterovirus $2 \mathrm{~A}^{\text {pro }}$ is the major antagonist of $S G$ formation.

Enterovirus $2 \mathrm{~A}^{\text {pro }}$ suppresses SG formation when introduced into recombinant EMCV containing an inactive stress antagonist. Overexpression of enteroviral proteases, followed by sodium arsenite treatment to induce SGs, may not accurately represent the role of the proteases in an enterovirus-infected cell. Given the essential role of the viral proteases in polyprotein processing and RNA replication, it is difficult to study their role in suppressing SG formation by introducing proteolytically inactivating mutations in the viral genome. We previously generated recombinant EMCVs harboring $2 \mathrm{~A}^{\text {pro }}$ and $3 \mathrm{C}^{\text {pro }}$ of different enteroviruses upstream of the leader protein, to study the proteolytic activity of $2 \mathrm{~A}^{\text {pro }}$ and $3 C^{\text {pro }}$ (21). Unfortunately, we could not use these viruses to study the effects of the enterovirus proteases on SG formation as EMCV actively suppresses SG formation. It has been suggested that $\mathrm{EMCV}_{3} \mathrm{C}^{\text {pro }}$ cleaves $\mathrm{G}_{3} \mathrm{BP}$ and that this prevents $\mathrm{SG}$ assembly, although this cleavage is observed only at very late stages of infection (10-12 hpi) (40). In contrast, we and others did not observe G3BP1 cleavage in cells infected with either EMCV or TMEV, and instead provided extensive evidence that the leader protein has an essential role in suppressing SG formation in cardiovirus-infected cells (11, 41, 42). As part of these studies, we generated $E M C V-\mathrm{L}^{\mathrm{Zn}}$, a mutant virus in which the leader protein is inactivated by mutations that disrupt its zinc finger domain and that, by consequence, can no longer suppress SG formation. To investigate the role of enterovirus proteases in suppressing SG
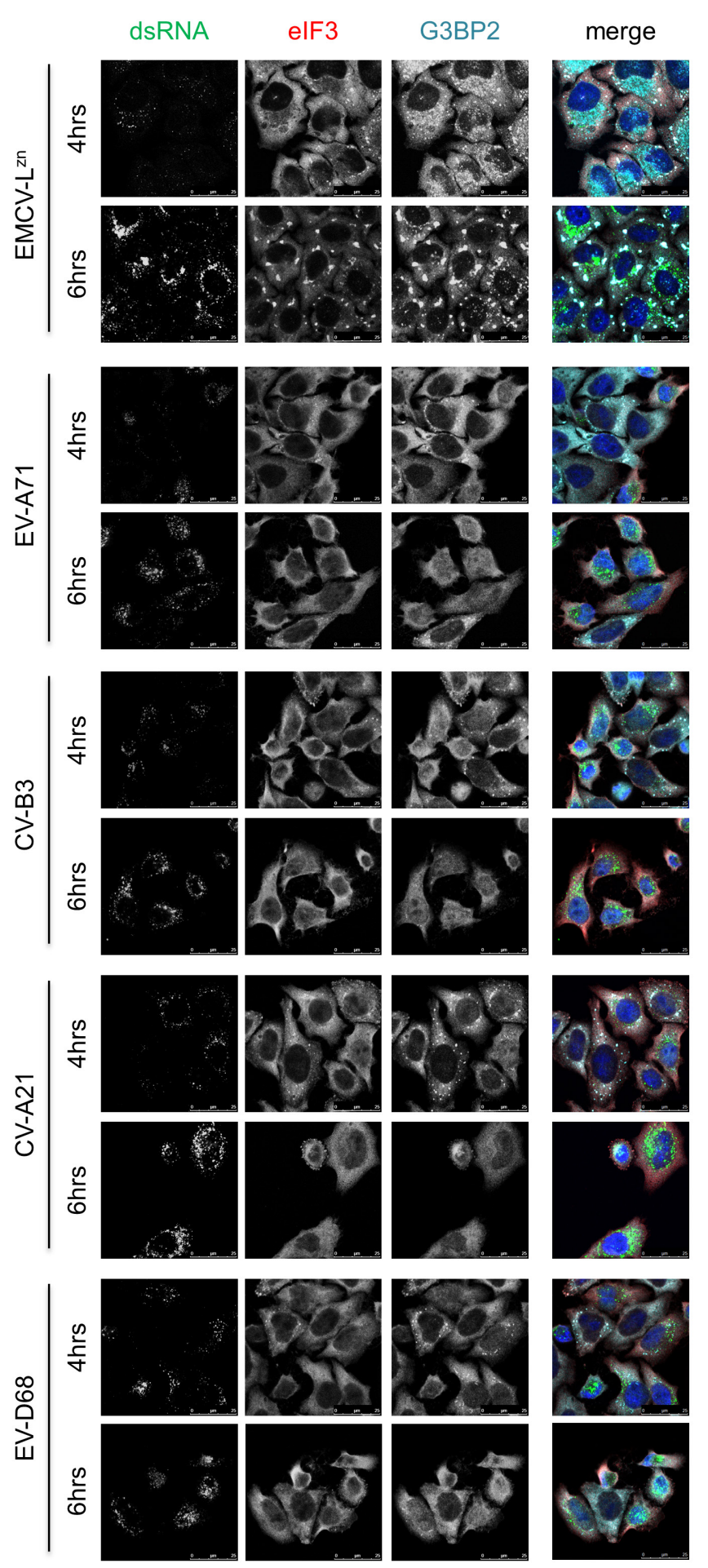

Figure 1: Multiple enteroviruses suppres SG formation. HeLa R19 cells were infected with EV-A71, CV-B3, CV-A21, EV-D68 or EMCV$\mathrm{Ln}^{\mathrm{Zn}}$ at $\mathrm{MOI} 10$ and cells were fixed at 4 or 6 hpi. Subsequently, immunofluorescence staining was performed for the SC markers $\mathrm{elF}_{3}$ and $\mathrm{C}_{3} \mathrm{BP} 2$, and viral replication was visualized by dsRNA staining. 
formation, we therefore inserted the genes encoding $C V-B 32 \mathrm{~A}^{\text {pro }}$ (EMCV- $2 \mathrm{~A}^{\text {pro }}$ ) or $\mathrm{CV}-\mathrm{B} 3$ ${ }_{3} C^{\text {pro }}\left(E M C V-3 C^{\text {pro }}\right)$ in EMCV- $\mathrm{L}^{\mathrm{Zn}}$ (Fig $\left.3 \mathrm{~A}\right)$. In parallel, we generated $\mathrm{EMCV}-\mathrm{L}^{\mathrm{Zn}}$ viruses encoding catalytically inactive mutants of the CV-B3 $2 \mathrm{~A}^{\text {pro }}$ (EMCV-2Am, mutation C109A) and $3 \mathrm{C}^{\text {pro }}\left(\mathrm{EMCV}-3 \mathrm{Cm}\right.$, mutation $\mathrm{C}_{147 \mathrm{~A}}$ ), allowing us to determine whether the effects of $2 \mathrm{~A}^{\text {pro }}$ and $3 \mathrm{C}^{\text {pro }}$ depend on their proteolytic activity. To ensure that $\mathrm{CV}-\mathrm{B} 32 \mathrm{~A}^{\mathrm{pro}}$ and $3 \mathrm{C}^{\text {pro }}$ are active in the context of EMCV- $\mathrm{L}^{\mathrm{Zn}}$ infection, we infected HeLa cells with the different chimeric EMCVs and performed western blot analysis of eIF4G, a well-known $2 \mathrm{~A}^{\text {pro }}$ target, or $\mathrm{G}_{3} \mathrm{BP} 1$, a well-known $3 \mathrm{C}^{\text {pro }}$ target (Fig $3 \mathrm{~B}$ ). Indeed, infection with $\mathrm{EMCV}-2 \mathrm{~A}^{\text {pro }}$, but not EMCV-2Am, resulted in cleavage of eIF4G, while infection with $E M C V-3 C^{\text {pro }}$ resulted in G3BP1 cleavage. No G3BP1 cleavage was observed in cells infected with either EMCV-L $\mathrm{Z}^{\mathrm{Zn}}$, which is consistent with our previous observations (11, 41, 42), or EMCV-3Cm.

A

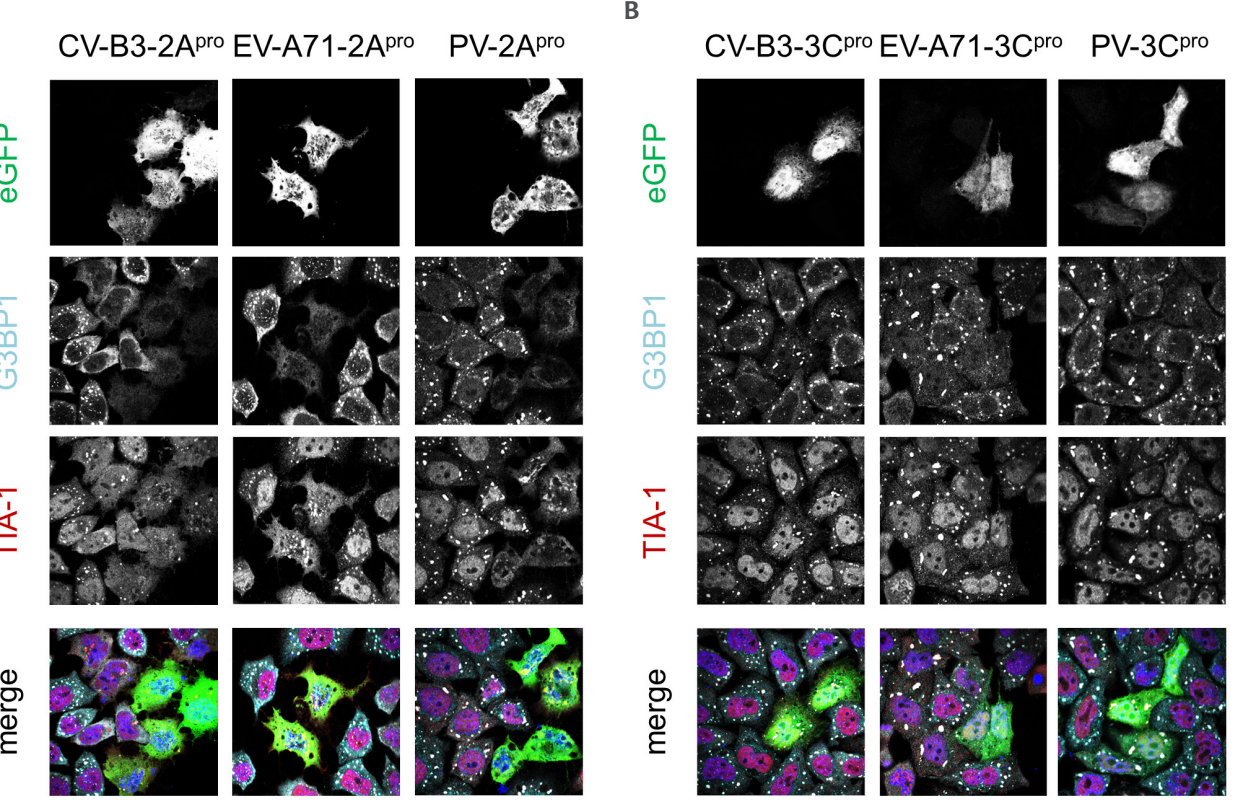

Figure 2: Overexpression of enterovirus $2 \mathrm{~A}^{\text {pro }}$ suppresses sodium arsenite-induced SGs. HeLa R19 cells were transfected with plasmids encoding the 2A $\mathrm{Apr}^{\mathrm{ro}}$ or $3 \mathrm{C}^{\mathrm{pro}}$ of CV-B3, EV-A71 or PV, N-terminally fused to ECFP. $16 \mathrm{~h}$ post transfection, cells were incubated with $500 \mu \mathrm{M}$ sodium arsenite for $30 \mathrm{~min}$. Subsequently, immunofluorescence staining was performed for the $\mathrm{SC}$ markers $\mathrm{C}_{3} \mathrm{BP} 1$ and $\mathrm{TIA}-1$

Next, we determined the ability of EMCV-2 $\mathrm{A}^{\text {pro }}$ and EMCV-3C $\mathrm{C}^{\text {pro }}$ to suppress SG formation. We infected HeLa cells with the different chimeric EMCVs, fixed the cells at 4 or $8 \mathrm{hpi}$ and performed immunofluorescence staining for the SG markers eIF3 and G3BP2, and dsRNA to identify the infected cells (Fig $3 \mathrm{C}$ ). Infection with EMCV-2A $\mathrm{A}^{\text {pro }}$ resulted in the formation of some small SGs at 4 hpi. Importantly, we did not observe these small SGs upon infection with the other chimeric EMCVs. This is consistent with previous reports that overexpression of $2 \mathrm{~A}^{\text {pro }}$ can induce formation of some small SGs $(31,37,38)$, most likely through its activity to cleave eIF4G and cause a host mRNA translation shut-off. Intriguingly, as infection with EMCV-2A $\mathrm{A}^{\text {pro }}$ progressed, these small SGs disappeared and we did not observe any SGs at 8 hpi. A similar pattern of SGs that first appear and subsequently disappear during the course of infection has frequently been reported for enteroviruses (34-37). Infection with EMCV-2Am did not induce small SGs at $4 \mathrm{hpi}$, nor did it suppress formation of larger SGs at $8 \mathrm{hpi}$. This indicates that the catalytic activity of $2 \mathrm{~A}^{\text {pro }}$ is needed for both the induction of small SGs at $4 \mathrm{hpi}$ and the subsequent suppression of the formation of larger SGs. In contrast to $2 \mathrm{~A}^{\text {pro }}$ expression (EMCV- $2 \mathrm{~A}^{\text {pro }}$ ), heterologous expression of $3 \mathrm{C}^{\text {pro }}\left(\mathrm{EMCV}-3 \mathrm{C}^{\mathrm{pro}}\right.$ ) had a small effect on SG formation. These data suggest that CVB3 $2 \mathrm{~A}^{\text {pro }}$, rather than $\mathrm{CVB} 3$ $3 \mathrm{C}^{\text {pro }}$, is the main antagonist of SG formation.

To test whether $2 \mathrm{~A}^{\text {pro }}$ is also the main stress antagonist of enteroviruses belonging to other species, we constructed EMCV-L $\mathrm{L}^{\mathrm{Zn}}$ viruses expressing either $2 \mathrm{~A}^{\text {pro }}$ or $3 \mathrm{C}^{\text {pro }}$ from EV-A71, CVA21, and EV-D68, and determined whether these chimeric viruses were able to suppress SG formation. HeLa cells were infected with these recombinant EMCV viruses and we visualized SGs by immunofluorescence staining. Subsequently, we quantified the number

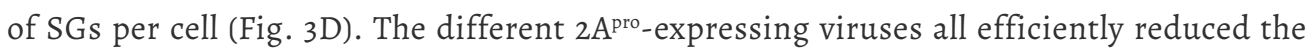

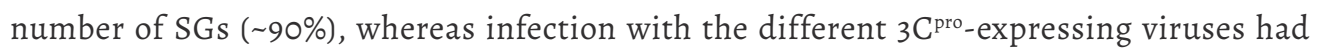
a less pronounced effect on the number of SGs ( $\sim 25-40 \%$ reduction). Overall, our data demonstrate that both enterovirus proteases have an effect on SG formation in HeLa cells, but that $2 \mathrm{~A}^{\text {pro }}$ is the main antagonist of $S G$ formation and that this function is conserved among enteroviruses belonging to species EV-A to EV-D.

Expression of $2 \mathrm{~A}^{\text {pro }}$ restores the impaired replication of EMCV-L ${ }^{\mathrm{Zn}}$. EMCV- $\mathrm{L}^{\mathrm{Zn}}$ replication in HeLa cells is predominantly restricted by the ISR (43). We next asked whether heterologous expression of enterovirus $2 \mathrm{~A}^{\mathrm{pro}}$, which efficiently suppressed SG formation, restored replication of EMCV- $\mathrm{L}^{\mathrm{Zn}}$. To investigate this, we compared the replication kinetics of EMCV$\mathrm{L}^{\mathrm{Zn}}, \mathrm{EMCV}-2 \mathrm{~A}^{\text {pro }}$ and $\mathrm{EMCV}-3 \mathrm{C}^{\text {pro }}$ (encoding the $\mathrm{CV}-\mathrm{B} 3$ proteases) in a multi-cycle infection. We infected HeLa cells - wt or deficient in PKR (PKR k.o.) - at a low multiplicity of infection and determined virus yields at $24 \mathrm{hpi}$ (Fig. 3E). In agreement with previous results $(39,43)$, we observed a strong defect in replication of EMCV- $\mathrm{L}^{\mathrm{Zn}}$ in wt cells, but not in PKR k.o. cells, confirming that replication in HeLa cells is predominantly restricted by the ISR. EMCV-2A $\mathrm{A}^{\text {pro }}$ replicated with similar efficiency in both cell lines, illustrating that heterologous expression of $2 \mathrm{~A}^{\text {pro }}$ rescued EMCV-L $\mathrm{L}^{\mathrm{Zn}}$ replication by countering the antiviral function of PKR-activated ISR. In contrast, expression of $3 \mathrm{C}^{\mathrm{pro}}$ failed to rescue EMCV- $\mathrm{L}^{\mathrm{Zn}}$ replication. Collectively, these data point to an important antagonistic function of $2 \mathrm{~A}^{\text {pro }}$ in counteracting PKR-induced SG formation in virus infected cells. 


\begin{tabular}{|c|c|c|c|c|}
\hline $\mathrm{VPg}-$ IRES - & \begin{tabular}{|l|l|}
$\begin{array}{l}2 A^{\text {pro }} \\
3 C^{\text {pro }}\end{array}$ & $L z n$ \\
\end{tabular} & P1 & P2 & P3 \\
\hline
\end{tabular}

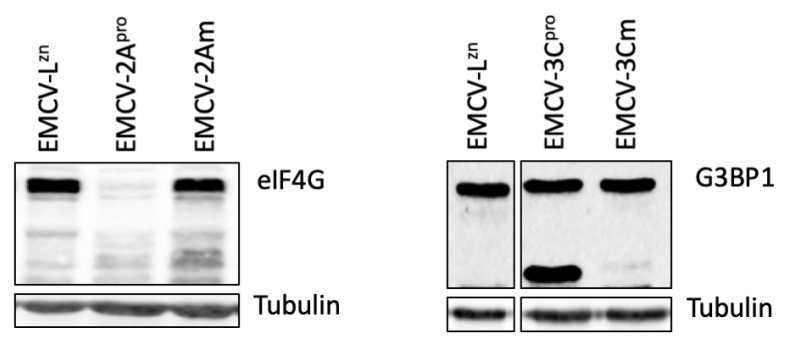

4 hpi

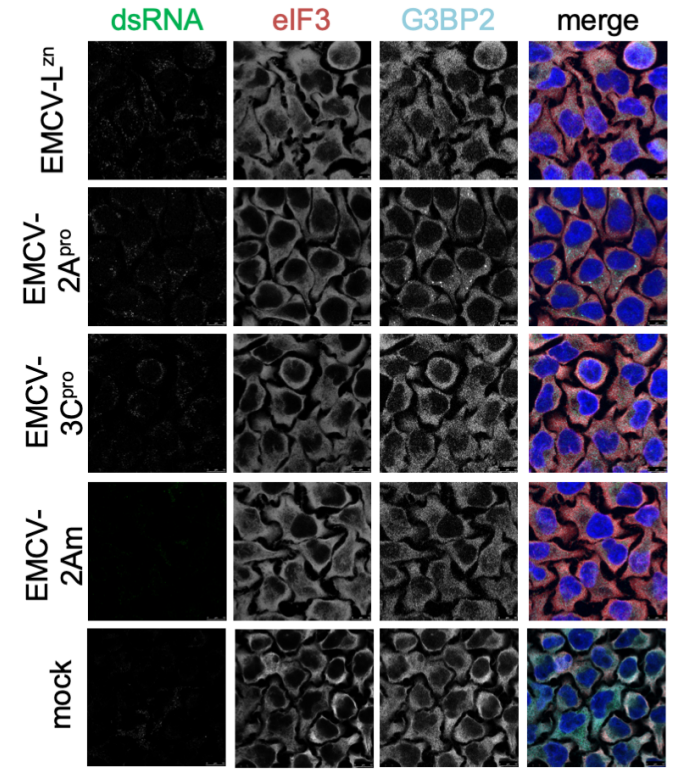

D

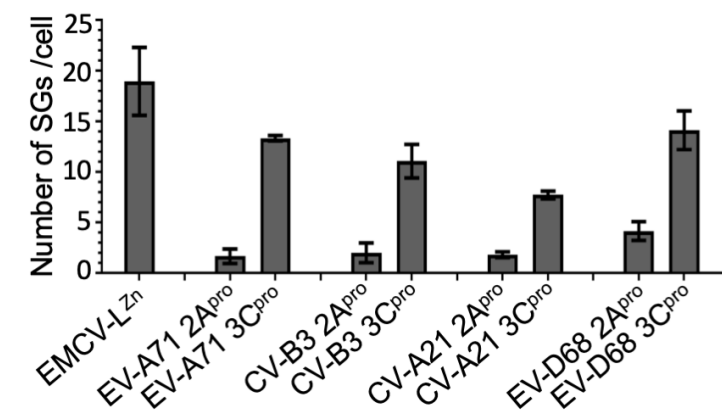

$\mathrm{E}$

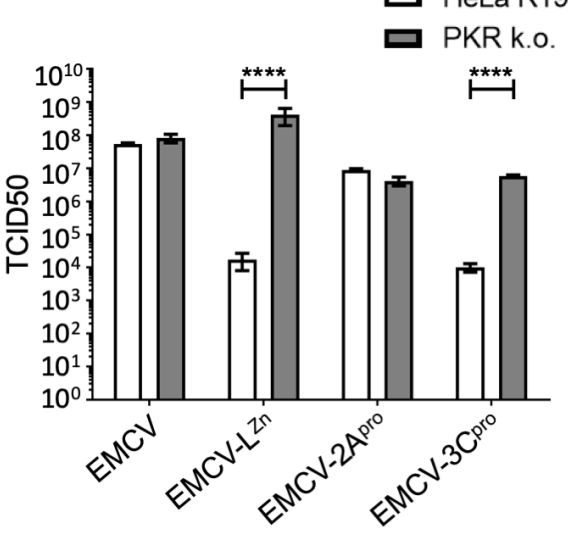

< Figure 3: Enterovirus 2A $A^{\text {pro }}$ suppresses SG formation in EMCV-infected cells. (A) Schematic representation of EMCV$\mathrm{L}^{\mathrm{Zn}}$ genome encoding $2 \mathrm{~A}^{\mathrm{pro}}$ or $3 \mathrm{C}^{\mathrm{pro}}$. The endogenous stress response antagonist (Leader) has been inactivated by point mutations in its $\mathrm{Zn}$-finger domain ( $\left.\mathrm{C}_{19} \mathrm{~A} / \mathrm{C}_{22} \mathrm{~A}\right)$, and subsequently the genes encoding $2 \mathrm{~A}^{\text {pro }}$ and $3 \mathrm{C}^{\mathrm{Cro}}$ were introduced at the $5^{\prime}$ end of the EMCV open reading frame. (B) HeLa R19 cells were infected with EMCV-LLn, EMCV-2A $A^{\text {pro }}$, EMCV-2Am, EMCV-3C $\mathrm{C}^{\mathrm{pro}}$ or EMCV-3Cm at MOl 10 and cells were lysed at $8 \mathrm{hpi}$. Lysates were subjected to western blot analysis for elF4C and $\mathrm{C}_{3} \mathrm{BP}_{1}$, and tubulin served as a loading control. (C) HeLa R19 cells were infected with EMCV-L $\mathrm{L}^{\mathrm{Zn}}$, EMCV-2A $\mathrm{APro}^{\text {, }}$ EMCV$2 \mathrm{Am}, \mathrm{EMCV}-3 \mathrm{C}^{\mathrm{pro}}$ or EMCV-3Cm at MOl 10 and cells were fixed at 4 or $8 \mathrm{hpi}$. Subsequently immunofluorescence staining was performed for the $\mathrm{SC}$ markers elF3 and $\mathrm{C}_{3} \mathrm{BP}$ 2. Viral replication was visualized by dsRNA staining. (D) HeLa R19 cells

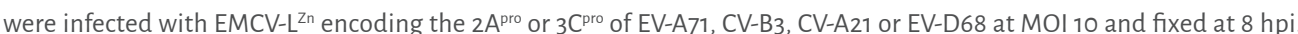
Subsequently immunofluorescence staining was performed for $\mathrm{C}_{3} \mathrm{BP}_{1}$ and the number of $\mathrm{SCs}_{\mathrm{s}} / \mathrm{cell}$ was analyzed for at least 50 cells per condition. (E) HeLa R19 cells, wt or deficient in PKR expression, were infected at MOI 0.01 with EMCV EMCV-L ${ }^{2 n}$, EMCV-2A $A^{\text {rro }}$ or EMCV-3 $3 \mathrm{Pro}^{\text {ro. }}$. At $24 \mathrm{hpi}$, samples were frozen and subjected to 3 freeze-thaw cycles. Subsequently, viral yield was determined by endpoint dilution on BHK21 cells.
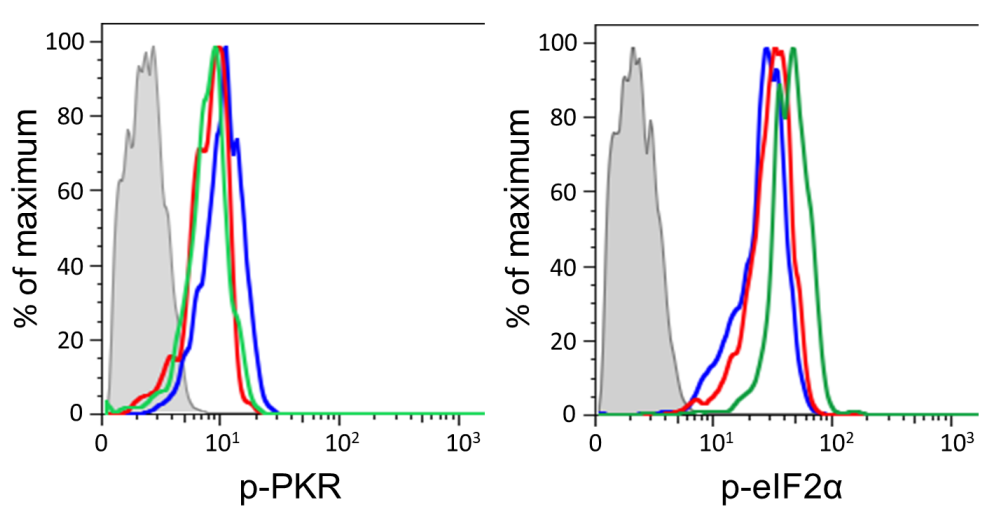

$\square$ mock

EMCV-2Apro

EMCV-2Am

Figure 4: Enterovirus $2 A^{\text {pro }}$ does not affect PKR signaling. HeLa R19 cells were infected with EMCV-L $L^{2 n}$, EMCV-2A pro or EMCV-2Am at MOI 10 and fixed at $8 \mathrm{hpi}$. Subsequently, cells were stained with antibodies against dsRNA and p-PKR or dsRNA and p-elF2 $\alpha$ for flowcytometric analysis. Craphs represent the level of p-PKR or p-elF2 $\alpha$ in dsRNA positive (infected) cells.

Enterovirus $2 \mathrm{~A}^{\text {pro }}$ does not affect PKR signaling. $2 \mathrm{~A}^{\text {pro }}$ depends on its catalytic activity to suppress SGs, which suggests that $2 \mathrm{~A}^{\text {pro }}$ cleaves a cellular factor to suppress SGs. $2 \mathrm{~A}^{\text {pro }}$ does not cleave known structural SG components like G3BP1, G3BP2 or TIA-1 $(35,37,38)$. To investigate whether $2 \mathrm{~A}^{\text {pro }}$ affects PKR signaling, we infected HeLa cells with EMCV-L ${ }^{2 \mathrm{n}}$, EMCV- $2 \mathrm{~A}^{\text {pro }}$ or EMCV-2Am and determined the levels of p-PKR and p-eIF $2 \alpha$ at $8 \mathrm{hpi}$ by flow cytometry (Fig 4). Infection with EMCV- $\mathrm{L}^{\mathrm{Zn}}$ activated the PKR signaling pathway, resulting in an increased level of p-PKR and p-eIF2 $\alpha$ relative to mock infected cells, consistent with previous results (43). Infection with EMCV-2A pro and EMCV-2Am resulted in similar increases in p-PKR and p-eIF2 $\alpha$ levels, demonstrating that $2 \mathrm{~A}^{\text {pro }}$ does not affect the PKR signaling pathway. Thus, it is unlikely that $2 \mathrm{~A}^{\text {pro }}$ suppresses $\mathrm{SG}$ formation by cleaving a factor involved in PKR signaling. How $2 \mathrm{~A}^{\text {pro }}$ suppresses SG formation remains to be established. 
Enterovirus $2 \mathrm{~A}^{\text {pro }}$ suppresses the induction of type I IFN gene transcription. In addition to the ISR, enteroviruses are known to suppress the induction of type I IFNs (11, 21-25). Indeed, infection of HeLa cells with EV-A71, CV-B3, CV-A21 or PV did not significantly induce expression of IFN- $\beta$ mRNA at 8 hr p.i. (Fig. $5 A$ ). Various studies have shown that both $2 \mathrm{~A}^{\text {pro }}$ and $3 \mathrm{C}^{\text {pro }}$ can cleave proteins in the RLR signaling pathway, and thus may be involved in suppressing IFN- $\beta$ gene transcription in infected cells $(21-27,30,44,45)$. To evaluate the effect of the $\mathrm{CV}-\mathrm{B} 3$ proteases on IFN- $\beta$ mRNA induction in infected cells, we infected HeLa cells with $E M C V-2 A^{\text {pro }}$ or EMCV- $3 C^{\text {pro }}$ and determined the induction of IFN- $\beta$ mRNA at 8 hpi by RT-qPCR analysis (Fig. 5B). As described previously $(3,11,39)$, EMCV-L $\mathrm{L}^{\mathrm{Zn}}$ failed to suppress the RLR signaling pathway and resulted in high IFN- $\beta$ mRNA levels. In contrast, infection with EMCV- $2 \mathrm{~A}^{\text {pro }}$ hardly induced IFN- $\beta$ mRNA levels, while infection with EMCV-2Am resulted in high IFN- $\beta$ mRNA levels, indicating that $2 \mathrm{~A}^{\text {pro }}$ - via its catalytic activity - suppresses IFN- $\beta$ gene transcription. Infection with EMCV- $3 \mathrm{C}^{\text {pro }}$ resulted in high levels of IFN- $\beta$ mRNA, indicating that $3 \mathrm{C}^{\text {pro }}$ is unable to suppress RLR-mediated signaling in HeLa cells. Collectively, these data suggest that enterovirus $2 A^{\text {pro }}$ is the viral protease that is responsible for suppressing IFN- $\beta$ gene transcription in infected HeLa cells. We also tested the effect of $2 \mathrm{~A}^{\text {pro }}$ and $3 \mathrm{C}^{\text {pro }}$ on JAK-STAT signaling by investigating the expression levels of ISGs. We determined the induction of ISG56 and MDA5 gene transcription via RT-qPCR analysis (Fig $5 \mathrm{C}$ and D). Consistent with the ability of $2 \mathrm{~A}^{\text {pro }}$ to suppress IFN- $\beta$ gene transcription, we did not observe an increase in the expression of these ISGs. $3 \mathrm{C}^{\text {pro }}$ had no effect on the induction of IFN- $\beta$ gene transcription (Fig. $5 \mathrm{~B}$ ), yet we observed a $\sim 10$-fold reduction in the expression of both ISG56 and MDA5, suggesting that $3 \mathrm{C}^{\text {pro }}$ affects JAK-STAT signaling rather than RLR signaling.

To determine whether the $2 \mathrm{~A}^{\text {pro }}$ of other enteroviruses also suppress IFN- $\beta$ gene transcription, we infected cells with EMCVs expressing $2 \mathrm{~A}^{\text {pro }}$ or $3 \mathrm{C}^{\text {pro }}$ from EV-A71, CV-A21, and EV-D68 and determined IFN- $\beta$ mRNA levels via RT-qPCR analysis (Fig. $5 \mathrm{E}$ ). Indeed, $2 \mathrm{~A}^{\mathrm{pro}}$ of these different enteroviruses, suppressed IFN- $\beta$ gene expression in the context of EMCV, while $3 \mathrm{C}^{\text {pro }}$ of these viruses did not. This demonstrates that the ability of $2 \mathrm{~A}^{\text {pro }}$ to suppress the induction of IFN- $\beta$ gene expression is conserved among EV-A to EV-D species members.

Inhibition of SG formation by $2 \mathrm{~A}^{\text {pro }}$ unlikely accounts for the inhibition of type I IFN gene transcription. It has been suggested that $S G$ formation is critical for the induction of type I IFN $(10,40)$. This raised the possibility that $2 \mathrm{~A}^{\text {pro }}$ suppresses the induction of IFN- $\beta$ mRNA via its ability to inhibit SG formation. To investigate the role of SGs in the induction of type I IFN we used PKR k.o. cells, which cannot form SGs. Two types of PKR k.o. MEFs have been described (46-48); one lacks the RNA binding domain (PKR RBD k.o.), while the other lacks the kinase domain (PKR KD k.o.) (Fig 6A). Interestingly, while it was shown that the PKR RBD k.o. cells fail to induce IFN expression (10), we previously observed only a 5 - to 10fold reduction in IFN $\beta$ mRNA levels in PKR KD k.o. MEFs (11). To better understand these
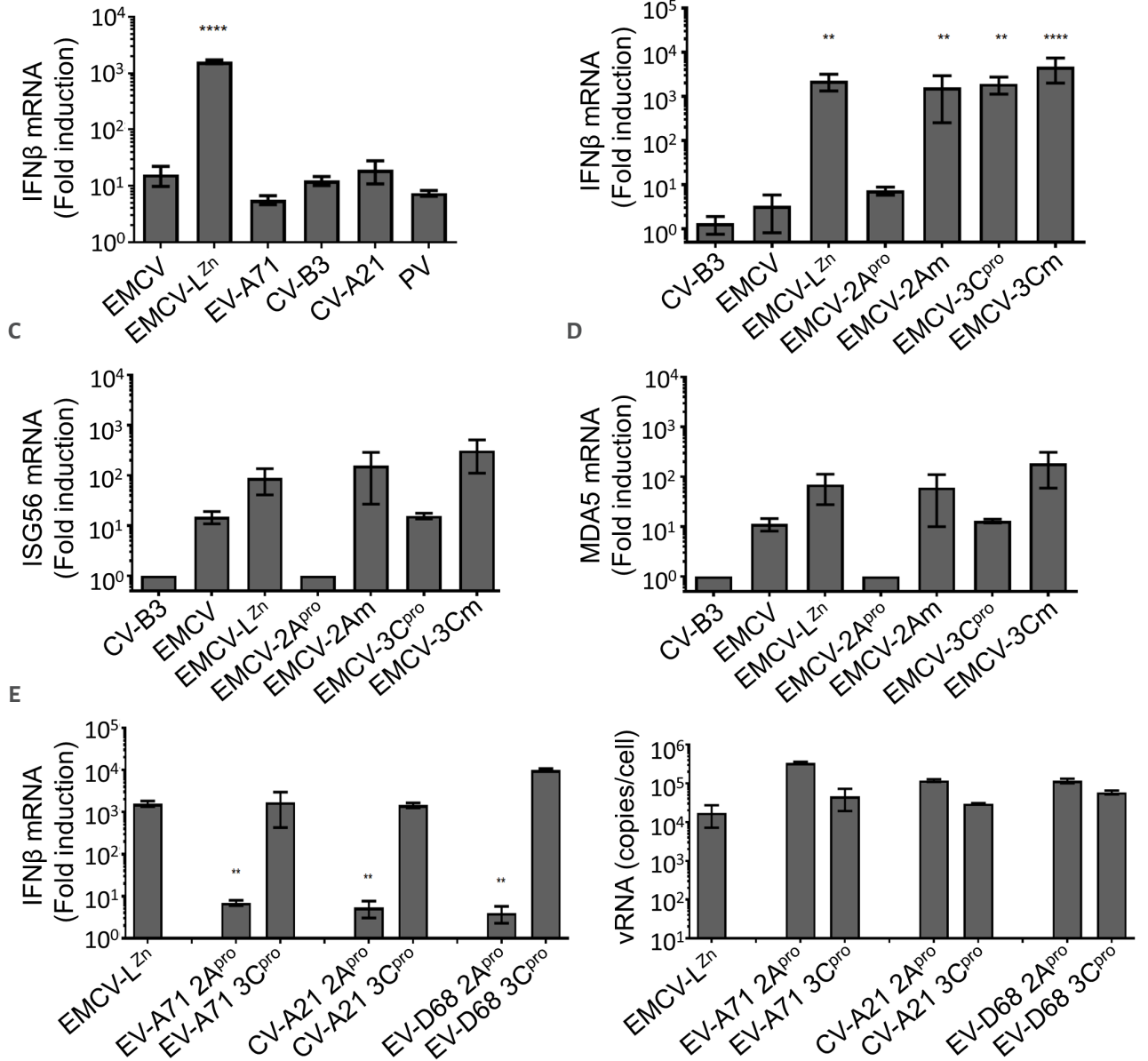

Figure 5: Enterovirus $2 A^{\text {pro }}$ suppresses the induction of type I IFN gene expression. (A) HeLa R19 cells were infected with the indicated viruses at $\mathrm{MOI} 10$ and cells were lysed at $8 \mathrm{hpi}$, total cellular RNA was isolated and RT-qPCR analysis was performed for IFN- $\beta$ and actin mRNA. (B, C, D) HeLa R19 cells were infected with CV-B3, EMCV, EMCV-L", EMCV-2A $A^{p \text { pa }}$ EMCV-2Am, EMCV-3 $C^{\text {pro }}$ or EMCV-3Cm at MOI 10 and cells were lysed at $8 \mathrm{hpi}$, total cellular RNA was isolated and RTqPCR analysis was performed for IFN- $\beta$, ISG56, MDA5 and actin MRNA. (E) HeLa R19 cells were infected with EMCV-

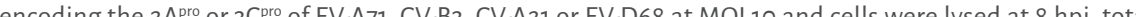
the fold induction of target gene mRNA levels compared to mock infected cells, after correction for actin mRNA levels, with the exception of EMCV vRNA, which is shown as a copy number per cell, calculated from a plasmid standard. Oneway ANOVA with Dunnet post-hoc test was used to determine statistical significance compared to EMCV (A-D) or EMCV

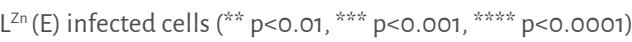


different results, we compared both types of PKR k.o. MEFs side-by-side under identical experimental conditions. We confirmed that both cell lines express truncated PKR using Western blot analysis (Fig 6B), and that both have lost the ability to form SGs (Fig 6C). Upon infection with EMCV-L $\mathrm{L}^{\mathrm{Zn}}$, the PKR RBD k.o. cells showed no IFN $\beta$ mRNA induction, while the PKR KD k.o. cells showed only a relatively small reduction (5- to 10-fold) in IFN $\beta$ mRNA levels. Why the two cell lines yield such different results remains to be elucidated. In addition to its role in the ISR, PKR has been suggested to play a role in the MDA5 signaling pathway (49). Possibly, the RBD is of greater importance for this function.
A

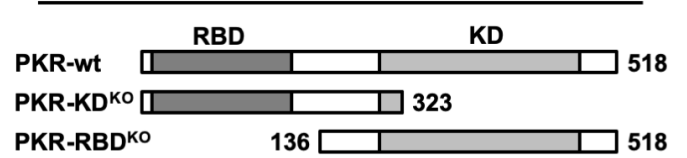

PKR-RBD ${ }^{K O}$

$136 \square$

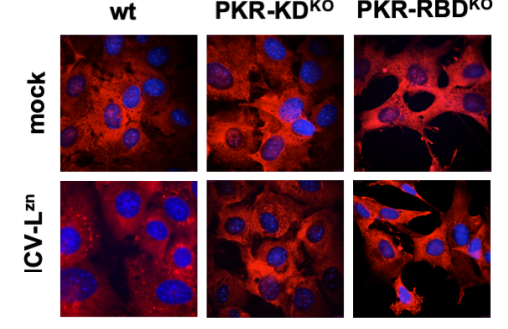

D
B $\frac{\text { PKR-KD KO }}{t+\frac{\text { PKR-RBD KO }}{4+1+}}$
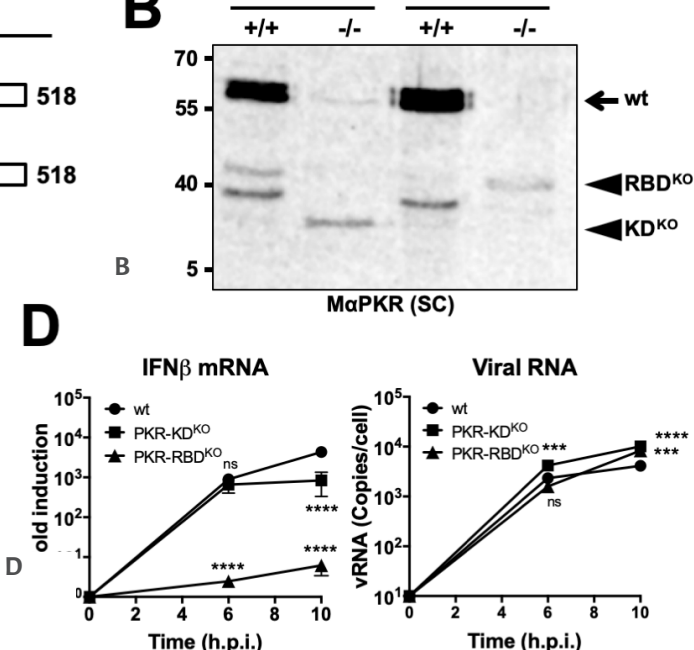

Figure 6: Two types of PKR k.o. MEFs are both deficient in SG formation but differ in IFN- $\beta$ induction. (A) Schematic representation of PKR gene and the two different types of PKR k.o. MEFs used in this study. In the PKR KD k.o. cells a premature stop codon was introduced before the kinase domain. In the PKR RBD k.o. cells the initial start codon was removed, resulting in a PKR that lacks the RNA binding domain. (B) PKR western blot analysis of lysates of PKR k.o. MEFs and the corresponding wt MEFs. (C) PKR k.o. and wt MEFs were infected with EMCV-L $\mathrm{Ln}^{\mathrm{n}}$ at MOI 10, fixed at $8 \mathrm{hpi}$, and stained for SC marker G3BP1. (D) PKR k.o. and wt MEFs were infected with EMCV-L ${ }^{\mathrm{Zn}}$ at MOI 10 and lysed at 6 or and $10 \mathrm{hpi}$. Total RNA was isolated and subjected to RT-qPCR analysis for IFN- $\beta$, actin mRNA and EMCV vRNA. IFN- $\beta$ mRNA is depicted as the fold induction of IFN- $\beta$ mRNA levels compared to mock infected cells, after correction for actin mRNA levels. EMCV vRNA is shown as a copy number per cell calculated from a plasmid standard. Two-way ANOVA with Bonferroni post-hoc test was used to calculate statistical significance $\left.{ }^{* * * *} p<0.001,{ }^{* * * * *} p<0.0001\right)$.

The results described here above did not provide a clear answer on the role of SGs in the induction of type I IFN. Therefore, we studied the role of SGs in IFN- $\beta$ induction via an alternative, PKR-independent, approach. To interfere with SG formation, we generated HeLa cells lacking expression of SG scaffolding proteins $\mathrm{G}_{3} \mathrm{BP} 1$ and/or G3B $\mathrm{BP} 2$ using CRISPR/ cas9 technology (Fig 7A). To characterize our newly generated cell lines, we determined whether they form SGs upon infection with $E M C V-\mathrm{L}^{\mathrm{Zn}}$ or addition of sodium arsenite (Fig 7B). While deletion of G3BP1 had a small effect on SG formation, G3BP1 and G3BP2 need to be simultaneously depleted ( $\mathrm{G}_{3} \mathrm{BP} / \mathrm{2}$ k.o.) to prevent $\mathrm{SG}$ formation, consistent with a previous siRNA-based study (50). To determine whether the PKR signaling pathway was affected by knocking out $\mathrm{G}_{3} \mathrm{BP} 1$ and/or $\mathrm{G}_{3} \mathrm{BP} 2$, we infected the different k.o. cell lines and performed Western blot analysis for PKR and p-PKR (Fig 7C). Infection with EMCV$\mathrm{L}^{\mathrm{Zn}}$ resulted in the phosphorylation of PKR in all cell lines, indicating that the signaling pathway was not affected. Next, we determined the induction of IFN- $\beta$ gene transcription upon infection with EMCV-L $\mathrm{L}^{\mathrm{Zn}}$ via RT-qPCR analysis (Fig $7 \mathrm{D}$ ). Only in the G3BP1/2 k.o. cells, which cannot form SGs, we observed 5- to 10-fold lower IFN $\beta$ mRNA levels at 6 and 8 hpi. This reduction is similar to what we previously observed in the PKR KD k.o. MEFs. Collectively our data suggest that, while SGs contribute to the induction of IFN- $\beta$ mRNA, they do not play a critical role. This implies that it is unlikely that the nearly complete inhibition of type I IFN gene transcription by $2 \mathrm{~A}^{\text {pro }}$ is due to its ability to suppress SG formation.

\section{Discussion}

To establish an infection, viruses must navigate an intricate network of antiviral host responses. Enteroviruses suppress both SG formation and the induction of IFN- $\alpha / \beta$. Since both enterovirus $2 \mathrm{~A}^{\text {pro }}$ and $3 \mathrm{C}^{\mathrm{pro}}$ are essential for viral polyprotein processing and virus replication, it is challenging to study the ability of these proteins to suppress SGs during enterovirus infection by impairing their function. Therefore, most previous work relies on overexpression data of $2 \mathrm{~A}^{\text {pro }}$ and $3 \mathrm{C}^{\text {pro }}$. However, overexpression of a viral protease may not accurately mimic its function during infection (21) and this may explain the conflicting results that have been reported on the role of these proteases in antagonizing the ISR and the IFN- $\alpha / \beta$ pathway. In this study, we demonstrated that EV-A71, CV-B3, CVA21, EV-D68 suppress SG formation and IFN- $\beta$ gene transcription in HeLa cells. To study the role of $2 \mathrm{~A}^{\text {pro }}$ and $3 \mathrm{C}^{\text {pro }}$ in suppressing SG formation and IFN- $\beta$ gene transcription, we introduced the proteases of $\mathrm{CV}-\mathrm{B} 3$ in a recombinant $\mathrm{EMCV}$ that was designed to efficiently activate antiviral responses (EMCV-L $\mathrm{L}^{2 \mathrm{n}}$ ). We demonstrated that $2 \mathrm{~A}^{\text {pro }}$ strongly inhibited SG formation and IFN- $\beta$ gene transcription in infected cells, suggesting that the cleavages mediated by $2 \mathrm{~A}^{\text {pro }}$ are more important for suppressing antiviral host responses than those mediated by $3 \mathrm{C}^{\text {pro }}$. Using the same system, we showed that $2 \mathrm{~A}^{\text {pro }}$ of EV-A71, CVA-21 and EV-D68 also suppressed SG formation and IFN- $\beta$ gene transcription, indicating that the identity of the antagonist is conserved among enteroviruses belonging to species EV-A to EV-D. 


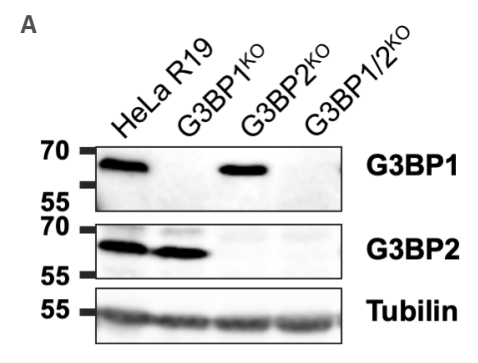

B
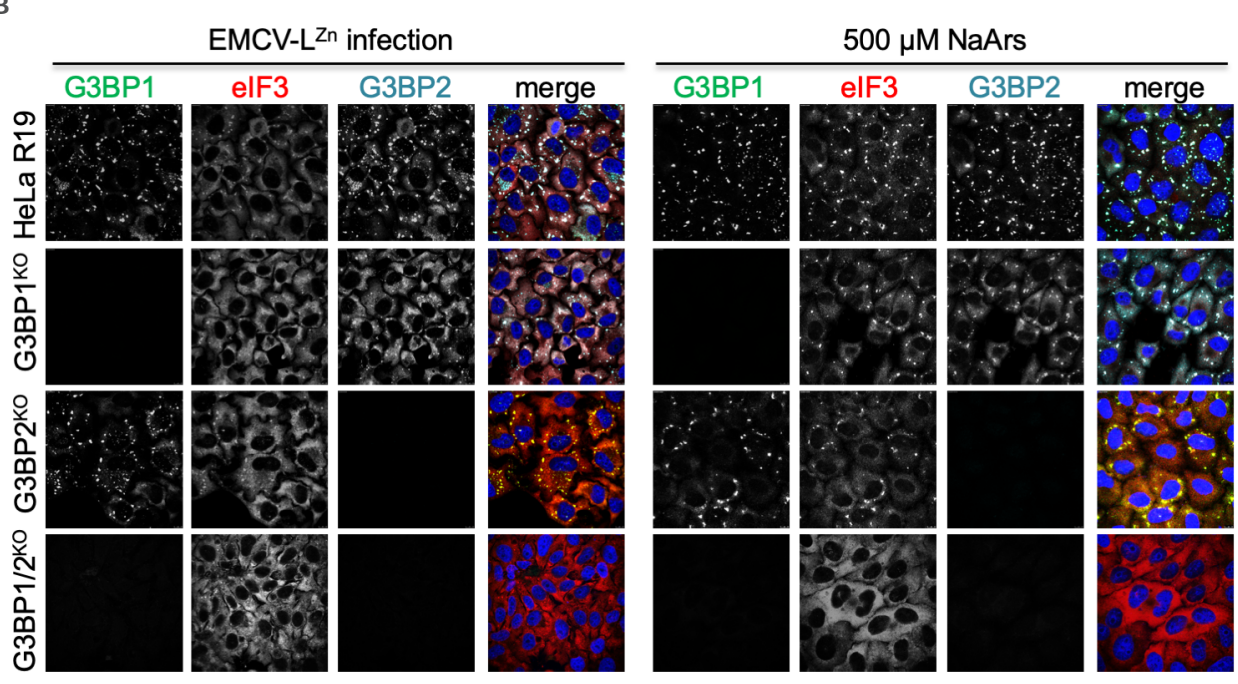

$\frac{\text { HeLa R19 }}{4 \text { G3ВP1ко }}$

G3BP2ко

G3BP1/2
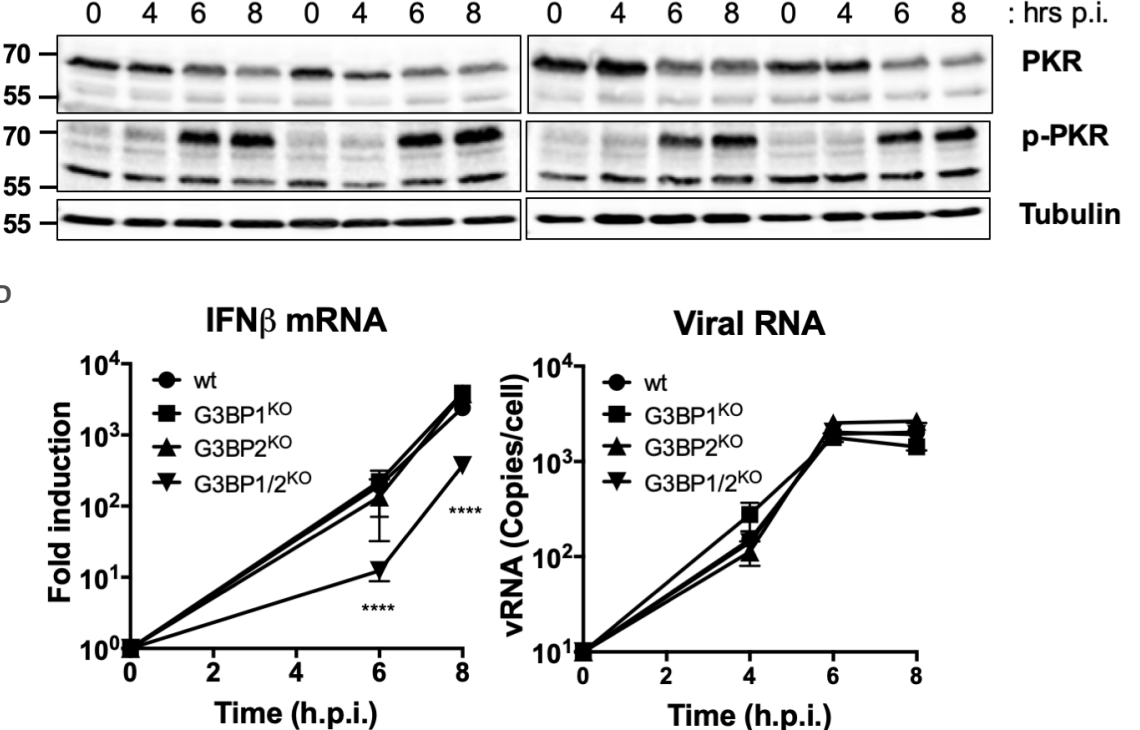

Viral RNA

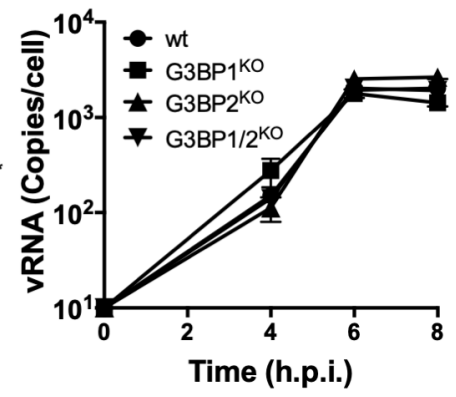

< Figure 7: SG formation-deficient HeLa $\mathrm{C}_{3} \mathrm{BP}_{1} / 2$ k.o. cells show only a small impairment in IFN- $\beta$ induction. (A) Lysates of $\mathrm{Hel}_{2} \mathrm{C}_{3} \mathrm{BP}_{1} \mathrm{k}_{0}, \mathrm{C}_{3} \mathrm{BP}_{2} \mathrm{k}_{0}$. and $\mathrm{C}_{3} \mathrm{BP}_{1} / 2 \mathrm{k} . \mathrm{o}_{0}$. cells were subjected to western blot analysis for $\mathrm{C}_{3} \mathrm{BP}_{1}$ $\mathrm{C}_{3} \mathrm{BP} 2$ and tubulin. (B) HeLa wt, $\mathrm{C}_{3} \mathrm{BP} 1$ k.o., $\mathrm{C}_{3} \mathrm{BP}_{2}$ k.o. and $\mathrm{C}_{3} \mathrm{BP}_{1} / 2$ k.o. cells were infected with EMCV-L $\mathrm{L}^{\mathrm{Zn}}$ at $\mathrm{MOl} 10$ or incubated with $500 \mu \mathrm{M}$ sodium arsenite. Cells were fixed at $8 \mathrm{hpi}$ or $30 \mathrm{~min}$ after addition of sodium arsenite, a and SCs were visualized by immunefluorescence staining for $\mathrm{C}_{3} \mathrm{BP}_{1}$, elF3 and $\mathrm{C}_{3} \mathrm{BP} 2$. (C) HeLa wt, $\mathrm{C}_{3} \mathrm{BP}_{1}$ k.o., $\mathrm{C}_{3} \mathrm{BP} 2$ k.o. and $\mathrm{C}_{3} \mathrm{BP}_{1} / 2$ k.o. cells were infected with $\mathrm{EMCV}-\mathrm{L}^{\mathrm{Zn}}$ at $\mathrm{MOl} 10$ and lysed at 4,6 and $8 \mathrm{hpi}$. Lysates were subjected to western blot analysis for PKR, phosphorylated PKR and tubulin. (D) HeLa wt, $\mathrm{C}_{3} B \mathrm{BP}_{1}$ k.o., $\mathrm{C}_{3} \mathrm{BP} 2$ k.o. and $\mathrm{C}_{3} \mathrm{BP}_{1} / 2$ k.o. cells were infected with EMCV-Lin at MOI 10 and lysed at 4, 6 and 8 hpi. Subsequently RNA was isolated and RT-qPCR analysis was performed for IFN- $\beta$, actin mRNA and EMCV vRNA. IFN- $\beta$ mRNA is depicted as the fold induction of IFN- $\beta$ mRNA levels compared to mockinfetedcells, attrcorrecton for a calculated from a plasmid standard. Two-way ANOVA with Bonferroni post-hoc test was used to calculate statistical

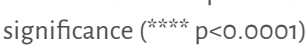

The ability of enteroviruses to suppress SG formation was initially attributed to the cleavage of $\mathrm{G}_{3} \mathrm{BP}_{1}$ by ${ }_{3} \mathrm{C}^{\mathrm{pro}}(34-36)$. In these studies, expression of a cleavage resistant $\mathrm{G}_{3} \mathrm{BP} 1\left(\mathrm{G}_{3} \mathrm{BP}_{1}{ }^{\mathrm{Q} 36 \mathrm{E}}\right)$ led to an increase in the number of $\mathrm{SGs}(34,35)$ and a small reduction in viral titers (34). We also observed that enterovirus $3 \mathrm{C}^{\text {pro }}$ had an effect on $S G$ formation, but enterovirus $2 \mathrm{~A}^{\text {pro }}$ had a much stronger inhibitory effect on the number of SGs. $2 \mathrm{~A}^{\text {pro }}$ strongly inhibited the number of SGs during overexpression of the protease, and in the context of EMCV infection. While this manuscript was in preparation, another study also reported the essential role of $2 \mathrm{~A}^{\text {pro }}$ in the suppression of SG formation (37). Importantly, in that study it was shown that an EV-A71 mutant containing an IRES between the P1 and P2 region and a catalytically inactive $2 \mathrm{~A}^{\text {pro }}$ was no longer able to suppress $S G$ formation (37). These data, combined with our data, strongly suggest that $2 \mathrm{~A}^{\text {pro }}$, rather than $3 \mathrm{C}^{\text {pro }}$, is the major enterovirus antagonist of the ISR.

How $2 \mathrm{~A}^{\text {pro }}$ suppresses the formation of SGs is unknown. Thus far, no cleavages of known SG components (e.g. G3BP1, G3BP2 and TIA-1) by $2 \mathrm{~A}^{\text {pro }}$ have been detected $(35,37,38)$. An alternative mechanism for viruses to prevent $S G$ formation is to interfere with the upstream PKR-dependent signaling $(43,51-55)$. However, this does not seem to be the case for enteroviruses. It has been reported that PKR and eIF2 $\alpha$ are phosphorylated during infection with several enteroviruses $(37,56-58)$, indicating that the viruses do not block activation of the ISR. Consistently, we did not observe an effect of $2 \mathrm{~A}^{\text {pro }}$ on the phosphorylation of PKR and eIF2 $\alpha$. Furthermore, $2 \mathrm{~A}^{\text {pro }}$ can inhibit arsenite-induced SG formation (Fig 2 and (37)), which is triggered via activation of another eIF2 $\alpha$ kinase. This suggests that $2 \mathrm{~A}^{\text {pro }}$ targets $S G$ assembly. While this manuscript was submitted, it was reported that SG formation relies on a newly identified interaction between eIF4GI and $\mathrm{G} 3 \mathrm{BP1}$, and that this interaction is targeted by $2 \mathrm{~A}^{\text {pro }}$ to block SG formation (59). Whether $2 \mathrm{~A}^{\text {pro }}$ also targets other protein-protein interactions underlying $S G$ formation remains to be established. Apart from this, it should be considered that the direct cleavage of eIF4G 
by $2 \mathrm{~A}^{\text {pro }}$ contributes to the suppression of SG formation. Cleavage of eIF4G will impair the recruitment of $40 S$ ribosomes to mRNAs, thereby altering the composition of mRNPs which, in turn, may affect their recruitment to SGs. The observation that $2 \mathrm{~A}^{\text {pro }}$ triggers $S G$ formation by cleavage of eIF4G $(31,37,38)$ seems counterintuitive, but these $2 \mathrm{~A}^{\text {pro-induced }}$ SGs are smaller in size and differ in composition from traditional SGs.Whether these small SGs fail to "grow" into traditional SGs because of the cleavage of eIF $4 \mathrm{G}$ by $2 \mathrm{~A}^{\text {pro }}$ or the ability of $2 \mathrm{~A}^{\text {pro }}$ to disrupt protein-protein interactions underlying $\mathrm{SG}$ formation, or both, remains unknown.

During enterovirus infection, several signaling molecules in the RLR pathway have been suggested to be cleaved by $2 \mathrm{~A}^{\text {pro }}$ (MDA5, and MAVS) and $3 \mathrm{C}^{\text {pro }}$ (RIG-I, MAVS, IRF7, and IRF9) $(21,23,26,27,29,44,45)$. There are controversial observations and the question remained which, if any, of the identified cleavages is important for inhibiting IFN- $\alpha / \beta$ gene transcription. For instance, several studies have shown that $3 \mathrm{C}^{\mathrm{pro}}$, when overexpressed, cleaves MAVS and suppresses IFN- $\alpha / \beta$ induction $(24,25)$, but we showed that $3 \mathrm{C}^{\text {pro }}$, when expressed by a recombinant EMCV, failed to cleave MAVS (21). Here, by infecting cells with EMCV-L $\mathrm{L}^{\mathrm{Zn}}$ encoding $2 \mathrm{~A}^{\text {pro }}$ or $3 \mathrm{C}^{\text {pro }}$, we were able to study the effect of these proteases on IFN- $\alpha / \beta$ mRNA levels in picornavirus-infected cells. Major advantages of this approach are that MDA-5 is activated by a natural ligand (i.e. EMCV dsRNA) and that the enterovirus protease ( $2 \mathrm{~A}^{\text {pro }}$ or $3 \mathrm{C}^{\text {pro }}$ ) is dynamically expressed during infection, similarly as in enterovirus-infected cells. Using these recombinant viruses, we demonstrate that enterovirus $2 \mathrm{~A}^{\text {pro }}$, but not $3 \mathrm{C}^{\text {pro }}$, suppresses the induction of IFN- $\alpha / \beta$ gene transcription in HeLa cells. That $3 \mathrm{C}^{\text {pro }}$, by cleaving RIG-I, has some role in suppressing IFN- $\alpha / \beta$ gene transcription in other cell types cannot be excluded. The exact mechanism(s) used by $2 \mathrm{~A}^{\text {pro }}$ to inhibit IFN- $\alpha / \beta$ gene transcription remains to be identified. We previously demonstrated that $2 \mathrm{~A}^{\text {pro }}$ cleaves MDA5 and MAVS (21). These cleavages are likely involved in the suppression of IFN- $\alpha / \beta$ induction, but we cannot formally exclude contributions

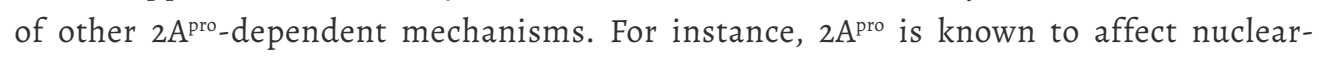
cytoplasmic transport via the cleavage of nuclear pore proteins (NUPs) (60-63), which could interfere with translocation of transcription factors (such as IRF3, IRF7 and NFxB) to the nucleus. The $2 \mathrm{~A}^{\mathrm{pro}}$-mediated inhibition of SG formation may also contribute to suppress IFN- $\alpha / \beta$ induction. In this study, we showed that cells that cannot form SGs (i.e. PKR KD k.o. MEFs and $\mathrm{G}_{3} \mathrm{BP} 1 / 2$ k.o. HeLa cells), showed a 5 - to 10 -fold reduction in IFN- $\beta$ gene transcription. The observation that heterologous expression of $2 \mathrm{~A}^{\text {pro }}$ during EMCV infection (EMCV-2A pro nearly completely blocked IFN- $\beta$ gene transcription ( $\sim 500-$ fold reduction) indicates that it is unlikely that suppression of $S G$ formation by $2 \mathrm{~A}^{\text {pro }}$ is the major contributing factor in the viral suppression of IFN- $\beta$ gene transcription.
Besides $2 \mathrm{~A}$ proteins as antagonists of antiviral responses, some picornaviruses (like EMCV) also encode an $\mathrm{L}$ protein. Many $2 \mathrm{~A}$ and $\mathrm{L}$ proteins have been shown to interfere with antiviral host responses, and have therefore collectively been referred to as "security proteins" (64). Our work demonstrates that for enteroviruses, which lack an L protein, $2 \mathrm{~A}^{\text {pro }}$ is essential for suppressing both SG formation and the induction of IFN- $\beta$ gene transcription, and we demonstrate that these two antagonizing functions are conserved in $2 \mathrm{~A}^{\text {pro }}$ of different enteroviruses belonging to species EV-A to EV-D. These data support the idea that $2 \mathrm{~A}^{\mathrm{pro}}$ is a major enteroviral "security protein".

\section{Materials and Methods}

Cells and viruses. HeLa R19 and BHK21 cells were maintained in Dulbecco's Modified Eagle's Medium (DMEM) supplemented with 10\% FCS (V/V). PKR KD and RBD k.o. MEFs have been described previously (46-48). HeLa R19 PKR k.o. have been described elsewhere (43). HeLa R19 G3BP1, G3BP2 and G3BP1/2 k.o. cells were made using CRISPR/ cas9 methodology as described previously $(43,65)$. G3BPI was targeted with gRNA sequences 5'-TAGTCCCCTGCTGGTCGGGC-3' and 5'-TATTACACACTGCTGAACC-3', and G3BP2 was targeted with gRNA sequences 5'-CGCCCTACAAGCAGCGG-3' and 5'AAGCTCCGGAATATTTACAC-3'. EV-D68, EV-A71 (BrCr), and CV-A21 (Coe) were obtained from the National Institute for Public Health and Environment (RIVM, The Netherlands). The poliovirus I Sabin reference strain was obtained from J. Martin (NIBSC, United Kingdom). All enteroviruses were passaged on HeLa R19 cells and subsequently concentrated by ultracentrifugation through a $30 \%$ sucrose cushion at $140,000 \times$ for 16 hours in a SW32Ti rotor and stored at $-80^{\circ} \mathrm{C}$. Recombinant $\mathrm{EMCVs}$ were generated by cloning the genes of interest into the XhoI/NotI restriction sites from the PM16.I-VFETQG-Zn infectious clone that was described previously (43), the Strep2 tag was omitted in the viruses used in this study. Viruses were recovered by transfection of run-off RNA transcripts into BHK-21 cells. Subsequently, viruses were concentrated by ultracentrifugation and stored at $-80^{\circ} \mathrm{C}$.

Expression plasmids. The $2 \mathrm{~A}^{\text {pro }}$ and $3 \mathrm{C}^{\text {pro }}$ genes from different enteroviruses were obtained by PCR on enterovirus viral RNA. The oligonucleotides used for these PCR reactions encode flanking XhoI and NotI restriction sites that were used to ligate the PCR products into the desired plasmids. The $3 \mathrm{C}^{\text {pro }}$ genes were ligated into the pcDNA-GFP vector, while the $2 \mathrm{~A}^{\text {pro }}$ genes were ligated into the PIRES-EGFP-MCS plasmid, both were described previously (42).

Antibodies. The following antibodies were used for IFA staining procedures: adsRNA (English\&Scientific consulting), $\alpha G_{3} B_{1} P_{1}$ (Aviva systems biology), $\alpha G_{3} B P_{2}$ (Bethyl laboratories), $\alpha e I F 3$ (SantaCruz) and $\alpha$ TIA-1 (SantaCruz). Alexa Fluor 488, Alexa Fluor 594 
and Alexa Fluor 647 conjugated secondary antibodies (Invitrogen) were used for detection. For flowcytometry staining we used the adsRNA (English\&Scientific consulting), $\alpha$-PKR (Abcam), $\alpha$ p-eIF2 $\alpha$ (Abcam) antibodies and Alexa488 or Alexa647 conjugated (Invitrogen) secondary antibodies. For western blot we used the antibodies: $\alpha G_{3} B P 1$ (Aviva systems

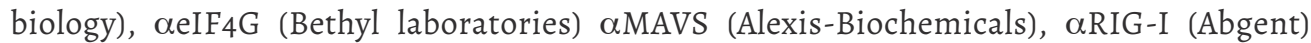
and atubulin (Sigma). Respective IRdye 680 or IRdye 800 conjugated secondary antibodies (LiCOR) were used for detection.

Immune Fluorescence Analysis (IFA). HeLa R19 cells were grown on $12 \mathrm{~mm}$ glass coverslips and the next day infected with the indicated viruses (MOI 10) or transfected with $1 \mu \mathrm{g}$ of the indicated plasmid using Fugene6 (Promega) according to manufacturer's instructions. At the indicated time points cells were washed in PBS before being fixed in $4 \%$ paraformaldehyde for $30 \mathrm{~min}$. Residual paraformaldehyde was washed away using PBS+10 mM Glycine. Cells were permeabilized in PBS+0.1\% Triton-X100 and subsequently incubated in blocking buffer (PBS+0.1\% Tween-20+3\% BSA) for 2 hours, all subsequent steps are performed in blocking buffer. Samples were incubated with primary antibody for 1 hour and incubated with secondary antibodies and DAPI for 30 min. After antibody incubations, samples were washed three times with PBS+0.1\% Tween-20. Before mounting coverslips on microscopy slides with FluorSafe (Calbiochem) they were washed once more in milliQ. Cells were examined by confocal microscopy (Leica SPE-II) and Leica Application Suite Advanced Fluorescence software (LAS-AF).

Quantification of SGs. The number of SGs and their surface area in $\sim 100$ cells per condition were analyzed by ImageJ software, using a combined total of 10-20 images. For each image, background signal was removed by creating a blurred duplicate and subtracting it from the original image. Subsequently, remaining diffuse (cytoplasmic) SG marker signal was removed via weak blurring, adjustment of the contrast settings and applying a black-andwhite threshold. In the resulting image, the number and average surface area of the SGs (shown in black on a white background) were quantified. The used macro is available upon request. Subsequent statistical analyses were performed using GraphPad Prism software. Error bars represent standard deviations, $\mathrm{p}$-values were calculated using one-way ANOVA analysis with Bonferroni post-hoc test (infections with recombinant EMCVs).

Flow cytometry analysis. HeLa R19 cells were seeded in 12-wells plates and the next day infected with the indicated viruses (MOI 10). Six hr p.i. cells were trypsinized and resuspended in FACS buffer (PBS + 1\% BSA). Cells were fixed for 30 minutes in $2 \%$ paraformaldehyde in FACS buffer and subsequently fixed in ice-cold methanol for 10 minutes. All subsequent steps are performed in FACS buffer. Cells were stained with primary antibodies for 1 hour. Subsequently cells were washed three times and incubated in secondary antibodies, in the dark, for $30 \mathrm{~min}$. Cells were washed three times and kept in $1 \%$ paraformaldehyde until analysis on FACS Canto II (BD biosciences) using BD FACS Diva software. Data analysis was performed using FlowJo software (TreeStar).

Western Blot analysis. HeLa R19 cells were seeded in $10 \mathrm{~cm}$ dishes and the next day infected with the indicated viruses (MOI 10). $8 \mathrm{hr}$ p.i. cells were released using trypsin, washed once in PBS and lysed in $100 \mu$ lysisbuffer (100 mM Tris pH 8.0, 1 mM EDTA, 50 $\mathrm{mM} \mathrm{NaCl}, 1 \% \mathrm{NP} 40$, protease inhibitor mix (Roche)). Post nuclear lysate was obtained by centrifugation at $15000 \mathrm{xg}$ at $4^{\circ} \mathrm{C}$ for $15 \mathrm{~min}$. The amount of total protein in the lysates was determined using BCA assay (ThermoFisher). $50 \mu \mathrm{g}$ protein from the cleared cell lysates were resolved using reducing sodiumdodecyl sulfate-polyacrylamide gel electrophoresis (SDS-PAGE) and transferred to $0.2 \mu \mathrm{m}$ nitrocellulose membranes by wet electrophoretic transfer. Membranes were incubated $\mathrm{th}$ in blocking buffer (PBS + $0.1 \%$ Tween $20+2 \% \mathrm{BSA}$ ) and successively incubated overnight with primary antibodies diluted in blocking buffer and then for $30 \mathrm{~min}$ with respective secondary antibodies diluted in blocking buffer. After antibody incubations, the membranes were washed three times with PBS+0.1\% Tween-20. Finally, membranes were washed once with PBS and scanned using an Odyssey Imager (Li-COR).

RT-PCR analysis. HeLa R19 cells were seeded in 24-wells plates and the next day infected with the indicated viruses at MOI 10, 8 hr p.i. cells were lysed and cellular RNA was isolated using total RNA isolation kit (Machery-Nagel) according to manufacturer's instructions. Reverse transcription was set up using TaqMan Reverse Transcription Reagents (Applied Biosystems) before performing qPCR analysis with SYBR green (Roche) as described previously (43).

\section{Acknowledgments}

The work was supported by a Vici grant (NWO-918.12.628) from the Netherlands Organization for Scientific Research. Martijn A. Langereis was supported by a Veni grant (NWO-863.13.008) and Linda J. Visser is supported by the NWO-graduate program Infection and Immunity (NWO-022.004.018), both from the Netherlands Organization for Scientific Research. 


\section{References}

1. Tapparel C, Siegrist F, Petty TJ, Kaiser L. 2013. Picornavirus and enterovirus diversity with associated human diseases. Infect Genet Evol 14:282-293.

2. Ventoso I, MacMillan SE, Hershey JW b., Carrasco L. 1998. Poliovirus 2A proteinase cleaves directly the eIF$4 \mathrm{G}$ subunit of eIF-4F complex. FEBS Lett 435:79-83.

3. Feng $\mathrm{Q}$, Hato SV V., Langereis MAA, Zoll J, Virgen-Slane R, Peisley A, Hur S, Semler BLL, van Rij RP, van Kuppeveld FJM. 2012. MDA5 Detects the Double-Stranded RNA Replicative Form in Picornavirus-Infected Cells. Cell Rep 2:1187-1196.

4. Wang JP, Cerny A, Asher DR, Kurt-Jones EA, Bronson RT, Finberg RW. 2010. MDA5 and MAVS mediate type I interferon responses to coxsackie B virus. J Virol 84:254-60.

5. Kuo R-L, Kao L-T, Lin S-J, Wang RY-L, Shih S-R. 2013. MDA5 Plays a Crucial Role in Enterovirus 71 RNAMediated IRF3 Activation. PLoS One 8:e63431.

6. Francisco E, Suthar M, Gale M, Rosenfeld AB, Racaniello VR. 2019. Cell-type specificity and functional redundancy of RIG-I-like receptors in innate immune sensing of Coxsackievirus B3 and encephalomyocarditis virus. Virology 528:7-18.

7. Ivashkiv LB, Donlin LT. 2014. Regulation of type I interferon responses. Nat Rev Immunol 14:36-49.

8. Goubau D, Deddouche S, Reis e Sousa C. 2013. Cytosolic Sensing of Viruses. Immunity 38:855-869.

9. Pakos-Zebrucka K, Koryga I, Mnich K, Ljujic M, Samali A, Gorman AM. 2016. The integrated stress response. EMBO Rep 17:1374-1395.

10. Onomoto K, Jogi M, Yoo JS, Narita R, Morimoto S, Takemura A, Sambhara S, Kawaguchi A, Osari S, Nagata K, Matsumiya T, Namiki H, Yoneyama M, Fujita T. 2012. Critical role of an antiviral stress granule containing RIG-I and PKR in viral detection and innate immunity. PLoS One 7:e43031.

11. Langereis MA, Feng $\mathrm{Q}$, van Kuppeveld FJ. 2013. MDA5 localizes to stress granules, but this localization is not required for the induction of type I interferon. J Virol 87:6314-25.

12. Kim WJ, Back SH, Kim V, Ryu I, Jang SK. 2005. Sequestration of TRAF2 into stress granules interrupts tumor necrosis factor signaling under stress conditions. Mol Cell Biol 25:2450-62.

13. Reineke $\mathrm{LC}$, Lloyd RE. 2015. The stress granule protein $\mathrm{G}_{3} \mathrm{BP}$ r recruits protein kinase $\mathrm{R}$ to promote multiple innate immune antiviral responses. J Virol 89:2575-89.

14. Reineke LC, Dougherty JD, Pierre P, Lloyd RE. 2012. Large G3BP-induced granules trigger eIF2 phosphorylation. Mol Biol Cell 23:3499-3510.

15. McCormick C, Khaperskyy DA. 2017. Translation inhibition and stress granules in the antiviral immune response. Nat Publ Gr 17:647-660.

16. Kedersha N, Ivanov P, Anderson P. 2013. Stress granules and cell signaling: More than just a passing phase? Trends Biochem Sci.

17. Humoud MN, Doyle N, Royall E, Willcocks MM, Sorgeloos F, van Kuppeveld F, Roberts LO, Goodfellow IG, Langereis MA, Locker N. 2016. Feline Calicivirus Infection Disrupts Assembly of Cytoplasmic Stress Granules and Induces $\mathrm{G}_{3} \mathrm{BP}$ Cleavage. J Virol 90:6489-501.

18. Panas MD, Varjak M, Lulla A, Eng KE, Merits A, Karlsson Hedestam GB, McInerney GM. 2012. Sequestration of $\mathrm{G}_{3} \mathrm{BP}$ coupled with efficient translation inhibits stress granules in Semliki Forest virus infection. Mol Biol Cell 23:4701-12.

19. Scholte FEM, Tas A, Albulescu IC, Žusinaite E, Merits A, Snijder EJ, van Hemert MJ. 2015. Stress granule components $\mathrm{G}_{3} \mathrm{BP}$ and $\mathrm{G}_{3} \mathrm{BP} 2$ play a proviral role early in Chikungunya virus replication. J Virol 89:4457-69.

20. Fros JJ, Domeradzka NE, Baggen J, Geertsema C, Flipse J, Vlak JM, Pijlman GP. 2012. Chikungunya virus nsP3 blocks stress granule assembly by recruitment of $\mathrm{G} 3 \mathrm{BP}$ into cytoplasmic foci. J Virol 86:10873-9.

21. Feng $\mathrm{Q}$ Langereis MA, Lork M, Nguyen M, Hato S V, Lanke K, Emdad L, Bhoopathi P, Fisher PB, Lloyd RE, van Kuppeveld FJM. 2014. Enterovirus 2Apro targets MDA5 and MAVS in infected cells. J Virol 88:3369-78.
22. Wang B, Xi X, Lei X, Zhang X, Cui S, Wang J, Jin Q, Zhao Z. 2013. Enterovirus 71 Protease 2Apro Targets MAVS to Inhibit Anti-Viral Type I Interferon Responses. PLoS Pathog 9:e1003231.

23. Drahos J, Racaniello VR. 2009. Cleavage of IPS-1 in cells infected with human rhinovirus. J Virol 83:11581-7.

24. Mukherjee A, Morosky SA, Delorme-Axford E, Dybdahl-Sissoko N, Oberste MS, Wang T, Coyne CB. 2011. The coxsackievirus B 3 Cpro protease cleaves MAVS and TRIF to attenuate host type I interferon and apoptotic signaling. PLoS Pathog 7:e1001311.

25. Pang L, Yuan X, Shao C, Li M, Wang Y, Wang H, Xie G, Xie Z, Yuan Y, Zhou D, Sun X, Zhang Q, Xin Y, Li D, Duan Z. 2017. The suppression of innate immune response by human rhinovirus C. Biochem Biophys Res Commun 490:22-28.

26. Lei $\mathrm{X}$, Xiao $\mathrm{X}, \mathrm{Xue} \mathrm{Q}$ Jin $\mathrm{Q}, \mathrm{He} \mathrm{B}$, Wang J. 2013. Cleavage of interferon regulatory factor 7 by enterovirus 7 $3 \mathrm{C}$ suppresses cellular responses. J Virol 87:1690-8.

27. Xiang Z, Liu L, Lei X, Zhou Z, He B, Wang J. 2016. 3C Protease of Enterovirus D68 Inhibits Cellular Defense Mediated by Interferon Regulatory Factor 7. J Virol 90:1613-21.

28. Hung HC, Wang HC, Shih SR, Teng IF, Tseng CP, Hsu JTA. 2011. Synergistic inhibition of enterovirus 71 replication by interferon and rupintrivir. J Infect $\mathrm{Dis}$.

29. Barral PM, Morrison JM, Drahos J, Gupta P, Sarkar D, Fisher PB, Racaniello VR. 2007. MDA-5 is cleaved in poliovirus-infected cells. J Virol 81:3677-84.

30. Barral PM, Sarkar D, Fisher PB, Racaniello VR. 2009. RIG-I is cleaved during picornavirus infection. Virology 391:171-176.

31. Zhang W, Zhang L, Wu Z, Tien P. 2014. Differential interferon pathway gene expression patterns in Rhabdomyosarcoma cells during Enterovirus 71 or Coxsackievirus A16 infection. Biochem Biophys Res Commun 447:550-555.

32. Lu J, Yi L, Zhao J, Yu J, Chen Y, Lin MC, Kung H-F, He M-L. 2012. Enterovirus 71 disrupts interferon signalin by reducing the level of interferon receptor 1. J Virol 86:3767-76.

33. Wang C, Sun M, Yuan X, Ji L, Jin Y, Cardona CJ, Xing Z. 2017. Enterovirus 71 suppresses interferon responses by blocking Janus kinase (JAK)/signal transducer and activator of transcription (STAT) signaling through inducing karyopherin- $\alpha 1$ degradation. J Biol Chem 292:10262-10274.

34. White JP, Cardenas AM, Marissen WE, Lloyd RE. 2007. Inhibition of Cytoplasmic mRNA Stress Granule Formation by a Viral Proteinase. Cell Host Microbe 2:295-305.

35. Fung G, Ng CS, Zhang J, Shi J, Wong J, Piesik P, Han L, Chu F, Jagdeo J, Jan E, Fujita T, Luo H. 2013. Production of a dominant-negative fragment due to $\mathrm{G}_{3} \mathrm{BP} 1$ cleavage contributes to the disruption of mitochondriaassociated protective stress granules during CVB3 infection. PLoS One 8:e79546.

36. Zhang Y, Yao L, Xu X, Han H, Li P, Zou D, Li X, Zheng L, Cheng L, Shen Y, Wang X, Wu X, Xu J, Song B, Xu S, Zhang $\mathrm{H}, \mathrm{CaO} \mathrm{H}$. 2018. Enterovirus 71 inhibits cytoplasmic stress granule formation during the late stage of infection. Virus Res 255:55-67.

37. Yang X, Hu Z, Fan S, Zhang Q, Zhong Y, Guo D, Qin Y, Chen M. 2018. Picornavirus 2A protease regulates stress granule formation to facilitate viral translation. PLOS Pathog 14:e1006901.

38. Dougherty JD, Tsai WC, Lloyd RE. 2015. Multiple poliovirus proteins repress cytoplasmic RNA granules. Viruses 7:6127-6140.

39. Hato S V, Ricour C, Schulte BM, Lanke KHW, de Bruijn M, Zoll J, Melchers WJG, Michiels T, van Kuppeveld JM. 2007. The mengovirus leader protein blocks interferon-alpha/beta gene transcription and inhibits activation of interferon regulatory factor 3. Cell Microbiol 9:2921-2930.

40. Ng CS, Jogi M, Yoo J-S, Onomoto K, Koike S, Iwasaki T, Yoneyama M, Kato H, Fujita T. 2013. Encephalomyocarditis virus disrupts stress granules, the critical platform for triggering antiviral innate mmune responses. J Virol 87:9511-22.

41. Borghese F, Michiels T. 2011. The Leader Protein of Cardioviruses Inhibits Stress Granule Assembly. J Viro $85: 9614-9622$ 
42. Visser LJ, Medina GN, Rabouw HH, de Groot RJ, Langereis MA, de Los Santos T, van Kuppeveld FJM. 2019. Foot-and-Mouth Disease Virus Leader Protease Cleaves $\mathrm{G}_{3} \mathrm{BP} 1$ and $\mathrm{G}_{3} \mathrm{BP}_{2}$ and Inhibits Stress Granule Formation. J Virol 93:e00922-18.

43. Rabouw HH, Langereis MA, Knaap RCM, Dalebout TJ, Canton J, Sola I, Enjuanes L, Bredenbeek PJ, Kikkert M, de Groot RJ, van Kuppeveld FJM. 2016. Middle East Respiratory Coronavirus Accessory Protein 4a Inhibits PKR-Mediated Antiviral Stress Responses. PLoS Pathog 12:e1005982.

44. Xiang Z, Li L, Lei X, Zhou H, Zhou Z, He B, Wang J. 2014. Enterovirus 683 C protease cleaves TRIF to attenuate antiviral responses mediated by Toll-like receptor 3. J Virol 88:6650-9.

45. Lei X, Sun Z, Liu X, Jin Q, He B, Wang J. 2011. Cleavage of the adaptor protein TRIF by enterovirus $713 \mathrm{C}$ inhibits antiviral responses mediated by Toll-like receptor 3. J Virol 85:8811-8.

46. Abraham N, Stojdl DF, Duncan PI, Méthot N, Ishii T, Dubé M, Vanderhyden BC, Atkins HL, Gray DA, McBurney MW, Koromilas AE, Brown EG, Sonenberg N, Bell JC. 1999. Characterization of transgenic mice with targeted disruption of the catalytic domain of the double-stranded RNA-dependent protein kinase, PKR. J Biol Chem 274:5953-62.

47. Yang YL, Reis LF, Pavlovic J, Aguzzi A, Schäfer R, Kumar A, Williams BR, Aguet M, Weissmann C. 1995. Deficient signaling in mice devoid of double-stranded RNA-dependent protein kinase. EMBO J 14:60956106.

48. Baltzis D, Li S, Koromilas AE. 2002. Functional Characterization of PKR Gene Products Expressed in Cells from Mice with a Targeted Deletion of the N terminus or C terminus Domain of PKR. J Biol Chem 277:3836438372.

49. Pham AM, Santa Maria FG, Lahiri T, Friedman E, Maít Signals for Type I IFN Induction. PLoS Pathog 12.

50. Kedersha N, Panas MD, Achorn CA, Lyons S, Tisdale S, Hickman T, Thomas M, Lieberman J, McInerney GM, Ivanov P, Anderson P. 2016. G3BP-Caprin1-USP10 complexes mediate stress granule condensation and associate with 40S subunits. J Cell Biol 212:845-60.

51. LU Y, WAMBACH M, KATZE MG, KRUG RM. 1995. Binding of the Influenza Virus NS1 Protein to DoubleStranded RNA Inhibits the Activation of the Protein Kinase That Phosphorylates the eIF-2 Translation Initiation Factor. Virology 214:222-228.

52. Chang HW, Watson JC, Jacobs BL. 1992. The E3L gene of vaccinia virus encodes an inhibitor of the interferoninduced, double-stranded RNA-dependent protein kinase. Proc Natl Acad Sci 89:4825-4829.

53. Feng Z, Cerveny M, Yan Z, He B. 2007. The VP35 protein of Ebola virus inhibits the antiviral effect mediated by double-stranded RNA-dependent protein kinase PKR. J Virol 81:182-92.

54. Cruz JLG, Sola I, Becares M, Alberca B, Plana J, Enjuanes L, Zuñiga S. 2011. Coronavirus Gene 7 Counteracts Host Defenses and Modulates Virus Virulence. PLoS Pathog 7:e1002090.

55. Li Y, Zhang C, Chen X, Yu J, Wang Y, Yang Y, Du M, Jin H, Ma Y, He B, Cao Y. 2011. ICP34.5 protein of herpes simplex virus facilitates the initiation of protein translation by bridging eukaryotic initiation factor zalpha (eIF2alpha) and protein phosphatase 1. J Biol Chem 286:24785-92.

56. Du Y, Bi J, Liu J, Liu X, Wu X, Jiang P, Yoo D, Zhang Y, Wu J, Wan R, Zhao X, Guo L, Sun W, Cong X, Chen L, Wang J. 2014. 3 Cpro of foot-and-mouth disease virus antagonizes the interferon signaling pathway by blocking STAT1/STAT2 nuclear translocation. J Virol 88:4908-20.

57. Black TL, Safer B, Hovanessian A, Katze MG. 1989. The cellular 68,000-Mr protein kinase is highly autophosphorylated and activated yet significantly degraded during poliovirus infection: implications for translational regulation. J Virol 63:2244-51.

58. Chang Y-H, Lau KS, Kuo R-L, Horng J-T. 2017. dsRNA Binding Domain of PKR Is Proteolytically Released by Enterovirus A71 to Facilitate Viral Replication. Front Cell Infect Microbiol 7:284

59. Yang X, Hu Z, Zhang $Q$, Fan S, Zhong Y, Guo D, Qin Y, Chen M. 2019. SG formation relies on eIF4GI-G3BP interaction which is targeted by picornavirus stress antagonists. Cell Discov 5:1.

60. Park N, Skern T, Gustin KE. 2010. Specific cleavage of the nuclear pore complex protein Nup62 by a viral protease. J Biol Chem 285:28796-805.
61. Park N, Katikaneni P, Skern T, Gustin KE. 2008. Differential targeting of nuclear pore complex proteins in poliovirus-infected cells. J Virol 82:1647-55.

62. Gustin KE, Sarnow P. 2001. Effects of poliovirus infection on nucleo-cytoplasmic trafficking and nuclear pore complex composition. EMBO J 20:240-9.

63. Watters K, Palmenberg AC. 2011. Differential processing of nuclear pore complex proteins by rhinovirus $2 A$ proteases from different species and serotypes. J Virol 85:10874-83.

64. Agol VI, Gmyl AP. 2010. Viral security proteins: counteracting host defences. Nat Rev Microbiol 8:867-78.

65. Langereis MA, Rabouw HH, Holwerda M, Visser LJ, van Kuppeveld FJM. 2015. Knockout of CGAS and STING Rescues Virus Infection of Plasmid DNA-Transfected Cells. J Virol 89:11169-73. 


\section{Chapter 6}

The Aichivirus leader protein inhibits the integrated stress response by preventing p-eIF2-mediated inhibition of eIF2B function

Linda J. Visser 1,**, Huib H. Rabouw ${ }^{1, * *}$, José G. Dekker ${ }^{1}$, Piero Giansanti 2,**, Jesús G. Saucedo ${ }^{1}$, Susanne G. van der Grein ${ }^{1}$, Martijn A. Langereis ${ }^{1}$, Albert J.R. Heck ${ }^{2}$, Raoul J. de Groot ${ }^{1}$, Frank J.M. van Kuppeveld ${ }^{1, *}$

${ }^{1}$ Virology Division, Department of Infectious Diseases and Immunology, Faculty of Veterinary Medicine, Utrecht University, Utrecht, The Netherlands

${ }^{2}$ Biomolecular Mass Spectrometry and Proteomics, Utrecht Institute for Pharmaceutical Sciences and Bijvoet Centre for Biomolecular Research, Utrecht University, Utrecht, The Netherlands

"Present Address: Chair of Proteomics and Bioanalytics, Technical University of Munich, Freising, Germany

"These authors share first authorship.

"Corresponding author. 


\section{Abstract}

Under conditions of stress, eukaryotic cells activate the integrated stress response (ISR) to temporarily block translation initiation, and restore cellular homeostasis. The ISR can be activated by four stress kinases, which, upon sensing specific stress stimuli, converge on phosphorylation of translation initiation factor eIF2. p-eIF2 sequesters the eIF2-specific guanidine exchange factor (GEF) eIF2B, which prevents eIF2•GDP/GTP exchange, an essential step in translation initiation. Many viruses avoid the detrimental effects of the ISR on viral protein synthesis by encoding specific ISR antagonists. We describe the mechanism through which aichivirus (AiV; family picornavirus, genus Kobuvirus KoV), interferes with the ISR. Whereas almost all ISR antagonist prevent or reverse eIF2 phosphorylation, AiV allows ongoing translation in the presence of high concentrations of p-eIF2, through the function of its leader protein (AiVL). AiVL binds eIF2B and blocks subsequent association of p-eIF2, thus preventing p-eIF2 from interfering with eIF2B GEF function. Besides AiVL, also the closely related mouse KoV leader, but not other KoV leaders, inhibits the ISR, suggestive of a relatively recent gain-of-function event. A mode of action similar to that of AiVL has been described for a phylogenetically unrelated coronavirus accessory protein (AcP10), which shares no similarity with AiVL. Thus, the ability to bind eIF2B and render it insensitive to p-eIF2 was acquired by at least two unrelated virus species through convergent evolution.

\section{Introduction}

To ensure efficient virus propagation, viruses evade several antiviral pathways within the infected host cell. One of these pathways, the integrated stress response (ISR), can be activated in response to a variety of intracellular danger signals including viral infection. The ISR mediates a temporal stop in translation, conserving energy and redirecting cellular resources towards resolving the stress situation (1). In mammalian cells, four ISRsensor proteins have been described. Protein kinase R (PKR) detects cytoplasmic dsRNA, and is considered the most important ISR sensor protein during virus infections (2). PKR-like ER-resident kinase (PERK) responds to ER-stress that causes misfolded proteins to accumulate in the ER lumen $(3,4)$. General control non-derepressible $(\mathrm{GCN} 2)$ is best known for its ability to detect nutrient deprivation and subsequent accumulation of uncharged tRNA molecules (5). Finally, Heme-responsive inhibitor (HRI) is activated by heme deficiency or the presence of toxic heavy metals $(6,7)$. Upon sensing their respective activating trigger, these proteins homodimerize and autophosphorylate, and subsequently phosphorylate translation initiation factor eIF2. Phosphorylated eIF2 (p-eIF2) tightly binds the eIF2-specific guanidine exchange factor (GEF) eIF2B, thereby inhibiting eIF2 recycling and preventing translation initiation. The stalled translation initiation complexes then condense to form cytoplasmic foci called stress granules (SGs).

In virus infected cells, ISR-mediated inhibition of translation severely represses production of viral proteins, and consequently the generation of progeny virus. Therefore, viruses have evolved multiple ways to circumvent the ISR, either by preventing eIF2 $\alpha$ kinase activation (Class 1 antagonist), by preventing eIF2 $\alpha$ phosphorylation (Class 2 antagonist), or by actively inducing dephosphorylation of p-eIF2 (Class 3 antagonist) (8). Recently, a novel mode of action was described for a coronavirus accessory protein, AcP10, that rescues cellular and viral translation without affecting eIF2 $\alpha$ phosphorylation by preventing the formation of p-eIF2•eIF2B complexes (Class 4 antagonist, (8)).

The picornavirus family encompasses a large group of non-enveloped positive-strand RNA viruses. This family includes many well-known pathogens of relevance to the health of humans and animals, such as enteroviruses (e.g. poliovirus), hepatoviruses (e.g. hepatitis A virus), and aphthoviruses (e.g. foot-and-mouth disease virus), and cardioviruses (e.g. encephalomyocarditis virus). Picornaviral genomes are 7,5-8 kb in length, and contain a single open reading frame that encodes a large polyprotein. This polyprotein is processed by viral proteases to yield the structural capsid proteins (VP1-4) and various proteins involved in replication ( $2 \mathrm{~A}-2 \mathrm{C}$ and $3 \mathrm{~A}-3 \mathrm{D})$. Besides this conserved set of proteins, several picornaviruses also encode a leader ( $\mathrm{L}$ ) protein upstream of the capsid coding region. $\mathrm{L}$ proteins are highly diverse in sequence, structure, and function, and are considered to be 
accessory proteins involved in optimizing virus replication, e.g. through suppression of the host cell's antiviral responses (9). The best known are the L protease of FMDV, which cleaves eIF4G to mediate host shut-off (10), and the L proteins of cardioviruses, which block IFN induction and stress responses $(11,12)$.

In 1991, a novel species of picornavirus, called aichivirus (AiV), was described as the causative agent of outbreaks of gastroenteritis in humans (13). Aichivirus was classified within a novel genus of picornaviruses, Kobuvirus (KoV). Since then, related KoVs have been found in various other mammalian species. KoVs are further subdivided into the lineages Aichi A (e.g. Aichivirus), Aichi B (e.g. Bovine KoV), and Aichi C (e.g. Porcine KoV) viruses, based on sequence analyses of their $3 \mathrm{CD}$-polymerase genes. In this study, we sought to determine whether and how KoVs evade inhibition by the ISR. We show that AiV actively blocks the ISR, both when activated in a PKR-dependent or HRI-dependent manner. Overexpression experiments demonstrate that the AiV-encoded L protein (AiVL) is a highly potent ISR antagonist in the absence of other viral proteins. Consistently, a recombinant AiV lacking a functional AiVL protein induces full-blown ISR activity, indicating that AiVL is the main, and possibly the sole ISR antagonist encoded by AiV. AiVL does not counteract the upstream signaling events of the ISR pathway, but rescues global translation in the presence of (high levels of) p-eIF2. This effect is mediated by its association to eIF2B, which prevents subsequent binding of $\mathrm{p}$-eIF2, and thus renders eIF2B insensitive to inhibition by p-eIF2. We show that the closely related mouse kobuvirus L protein (M-KoVL) has a similar function, but that other tested KoVLs do not, suggesting that the ISR antagonist function was obtained through a gain-of-function event relatively late in the KoVL evolutionary trajectory. AiVL's mode of action strongly resembles that of Beluga-whale coronavirus AcP10, and defines it as a Class 4 ISR antagonist (8). Thus, the ability to bind eIF2B and prevent inhibition by p-eIF2 independently developed within at least two unrelated viruses, in non-homologous proteins, through convergent evolution.

\section{Results}

Aichivirus prevents ISR activity in infected cells. To test whether AiV suppresses the ISR in infected cells, we assessed SG formation in AiV-infected cells. For this, we first established replication kinetics in infected Vero cells. A single-step growth curve, in which we quantified the production of progeny infectious virus at the indicated time points pi. (Fig $1 \mathrm{~A})$, indicated that most virus is produced between 6 and $10 \mathrm{~h}$ pi. In line with these data, an immunofluorescence assay (IFA) shows large quantities of dsRNA, indicative of active virus replication, at the same time points (Fig $1 B$ ). Interestingly, despite the appearance of dsRNA, which could activate the ISR sensor protein PKR, infected cells were consistently devoid of SGs throughout the infection cycle, up to and including the moment they started displaying cytopathic effects at $10 \mathrm{~h}$ pi. These data provided first indication that AiV counteracts the ISR. This was further corroborated by the observation that AiV-infected cells failed to assemble SGs in response to arsenite treatment, which activates the eIF2 $\alpha$ kinase HRI (Fig 1C).

The Aichivirus-encoded $\mathrm{L}$ protein rescues cellular translation in the presence of p-eIF2. Multiple picornavirus L proteins have been shown to repress cellular antiviral responses, including the ISR $(11,12,14,15)$. To test whether the AiV Leader protein (AiVL) is responsible for inhibiting the ISR in AiV-infected cells, we transiently overexpressed EGFP-tagged AiVL in absence of other viral proteins, and assessed SG formation in response to (i) the pEGFP plasmid DNA transfection itself, which induces PKR- and p-eIF2-dependent stress in some of the transfected cells (Fig $2 \mathrm{~A},(16,17)$ ), (ii) treatment with sodium arsenite, which induces HRI- and p-eIF2-dependent stress in all treated cells (Fig 2B), or (iii) treatment with pateamine A, which blocks translation independently of p-eIF2 (Fig $2 \mathrm{C}$ ). Expression of AiVL prevented p-eIF2-mediated SG formation (Fig $2 \mathrm{~A}$ and $2 \mathrm{~B}$ ), but not p-eIF2-independent SG formation induced by pateamine A (Fig $2 \mathrm{C}$ ), indicating that AiVL is indeed a functional ISR antagonist. Furthermore, these data suggest that AiVL targets a downstream step in the ISR pathway that is shared by transfection-induced and arseniteinduced ISR activity. To determine which step of the ISR is targeted by AiVL, we performed flow cytometry analyses to quantify p-eIF2 $\alpha$ levels and active translation rates in cells overexpressing AiVL. pEGFP plasmid DNA transfection induces elevated levels of p-eIF2 in a portion of the transfected cells, while the remainder of the cells are unstressed $(16,17)$, resulting in a mixed population of transfected cells with either high (shown in red) or low (shown in green) levels of p-eIF2 $\alpha$ (Fig. 2D). The level of p-eIF2 $\alpha$ was similar between AiVL expressing cells and cells expressing negative control EGFP, suggesting that AiVL neither impacts eIF2 $\alpha$ phosphorylation, nor induces eIF2 $\alpha$ dephosphorylation. As expected, EGFP expressing cells with high p-eIF2 $\alpha$ levels displayed impaired translation rates (as measured by puromycin labeling) (Fig $2 \mathrm{D}$, lower panels). In contrast, in AiVL expressing cells p-eIF2 $\alpha$ had no detectable effect on translation, suggesting that AiVL renders cellular translation insensitive to eIF2 $\alpha$ phosphorylation.

A putative $\mathrm{Zn}$-finger domain is essential for ISR antagonism by AiVL. To obtain a nonfunctional AiVL mutant, we performed alanine screening, and substituted specific tryptophans, histidines, cysteines, or potential phosphorylation sites by alanines (Fig $3 \mathrm{~A}$, residues indicated in red). The mutant proteins were overexpressed as EGFP fusion proteins and tested for their ability to inhibit arsenite-induced SG formation using IFA. Out of the twenty mutations tested, five $(\mathrm{H} 20 \mathrm{~A}, \mathrm{H} 24 \mathrm{~A}, \mathrm{H} 40 \mathrm{~A}, \mathrm{C} 53 \mathrm{~A}$, and $\mathrm{C} 56 \mathrm{~A})$ rendered the AiVL non-functional, three of which (H24A, C53A, C56A) did so without significantly 
impacting AiVL-expression level or its intracellular localization (Fig 3B). A full overview of the mutants tested is given in supplementary figure S1. Flow cytometry analysis confirmed that the mutant AiVL proteins failed to rescue translation in the presence of elevated p-eIF2 $\alpha$ levels (Fig $3 \mathrm{C}$ ). Interestingly, two of the inactivating mutations affected residues that make up an in silico predicted $\mathrm{Zn}$-finger ribbon (C53 and C56). Most Zn-fingers consist of two $\mathrm{C} / \mathrm{H}-\mathrm{X}_{2-4}-\mathrm{C} / \mathrm{H}$ motifs, which mediate a quadruple interaction with a divalent cation such as $\mathrm{Zn}^{2+}$. In the case of AiVL, the second $\mathrm{C} / \mathrm{H}-\mathrm{X}_{2-4}-\mathrm{C} / \mathrm{H}$ motif was not predicted in silico, but may consist of $\mathrm{H} 2 \mathrm{O}$ and $\mathrm{H} 24$, since both these residues were also found to be essential for AiVL's function as ISR antagonist (Fig 3 B and S1).

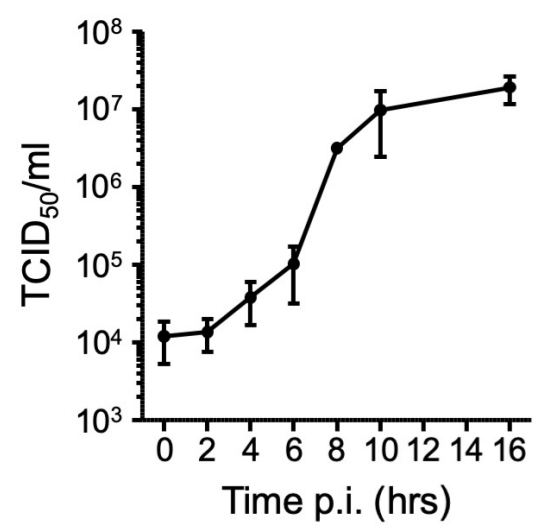

C

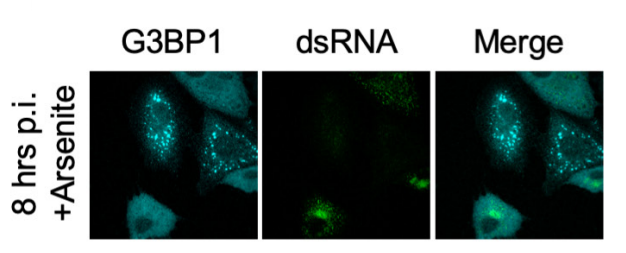

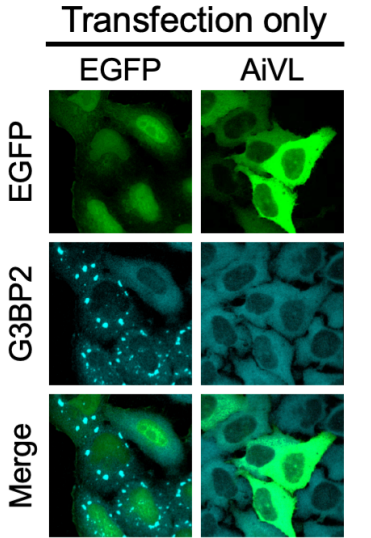
EGFP AiVL-EGFP
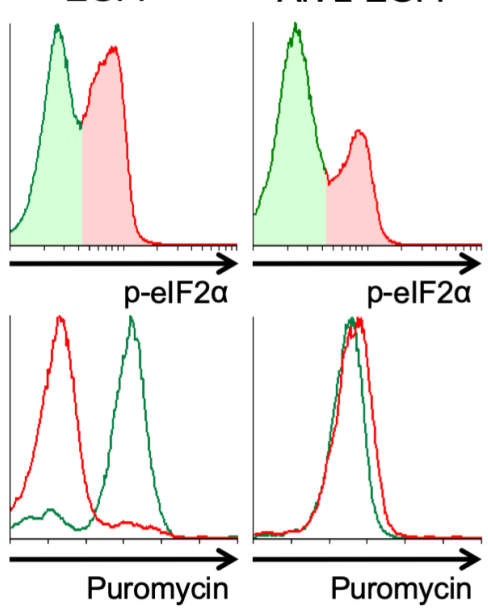

p-elF2alow

p-elF2a $a^{\text {high }}$

Figure 2: AiVL rescues translation in the presence of p-elF2. Hela cells were transfected with plasmids encoding ECFP or AiVL-ECFP. Subsequently, SC formation was monitored by immunofluorescence in cells without additional treatme were transfected with PECFP or pECFP-AiVL Next min. Subsequently, cells were fixed and analyzed by flow cytometry for p-elF2 $\alpha$ content and the level of puromycin incorporation. Shown are cells positive for ECFP, with either low p-elF2 $\alpha$ levels (indicated in green) or high p-elF2 $\alpha$ levels (indicated in red)

Figure 1: Aichivirus counteracts the ISR. Vero cells were infected with aichivirus at a multiplicity of infection (MOI) of 2. (A) At the indicated time points, progeny infectious virus particles were harvested and quantified by end-point titrations. (B) At the indicated time-points, cells were fixed and stress granule (SC) formation was analyzed by immunofluorescence using an antibody directed against SC marker $\mathrm{C}_{3} \mathrm{BP}$ 1. dsRNA staining was used to identify cells that were actively replicating AiV. (C) Aichivirus-infected cells were treated with $1 \mathrm{mM}$ arsenite from 7 hrs pi. until 8 hrs pi., and SC formation was analyzed as in (B). 


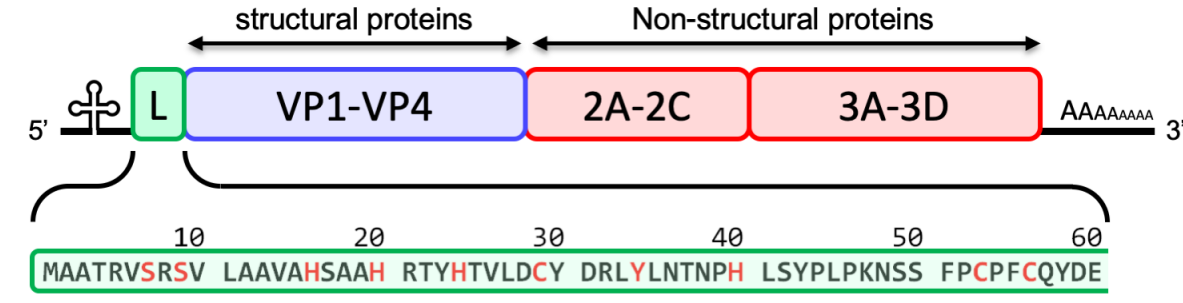

\begin{tabular}{rrrrrr}
70 & 80 & 90 & 100 & 110 & 120 \\
\hline QNEVLSSPESL CGEGAEPCWK CSQDKPRRKY NTTPPEDWLY & DSDVQSWFYP & ETYYSDLQQK \\
\hline
\end{tabular}

\begin{tabular}{rrrrr}
130 & 140 & 150 & 160 & 170 \\
\hline FFDKLALLSL & PGAYQAKTPE & ERALAGALTQ & LLNFPSTPPL & TLPTTNLQRQ \\
\hline
\end{tabular}
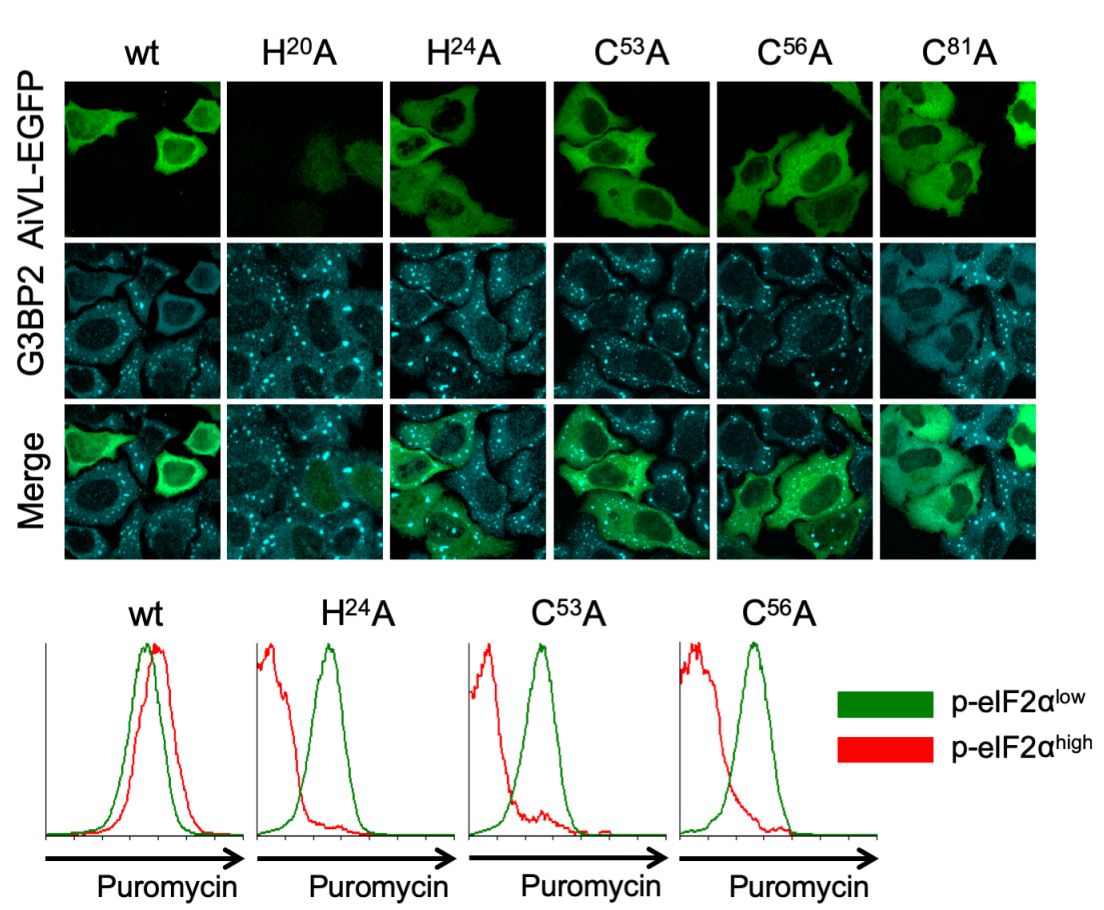

Figure 3: A putative Zn-finger domain is essential for ISR antagonism by AiVL. (A) Schematic representation of the AiV genome. The position of the gene encoding AiVL is indicated in green. The amino acid sequence of AiVL is shown in the bottom panel, and each residue that was mutated in the alanine screening approach is indicated in red. (B) The AiVL mutants were overexpressed in cells, and their ability to prevent SC formation upon arsenite treatment was analyzed by immun overexpessed in cells, and their ability to prevent $\mathrm{SC}$ formation upon arsenite treatment was analyzed shown. See Fig $S_{1}$ for a using antibodies directed against $\mathrm{C}_{3} \mathrm{BP}_{1}$. A small selection out of the total panel of mutants is overexpressed, and after overnight incubation, active translation was labeled using $20 \mathrm{ug} / \mathrm{ml}$ puromycin for $15 \mathrm{~min}$. Cells were subsequently analyzed by flow cytometry for active translation rates as described in Fig $2 \mathrm{D}$.
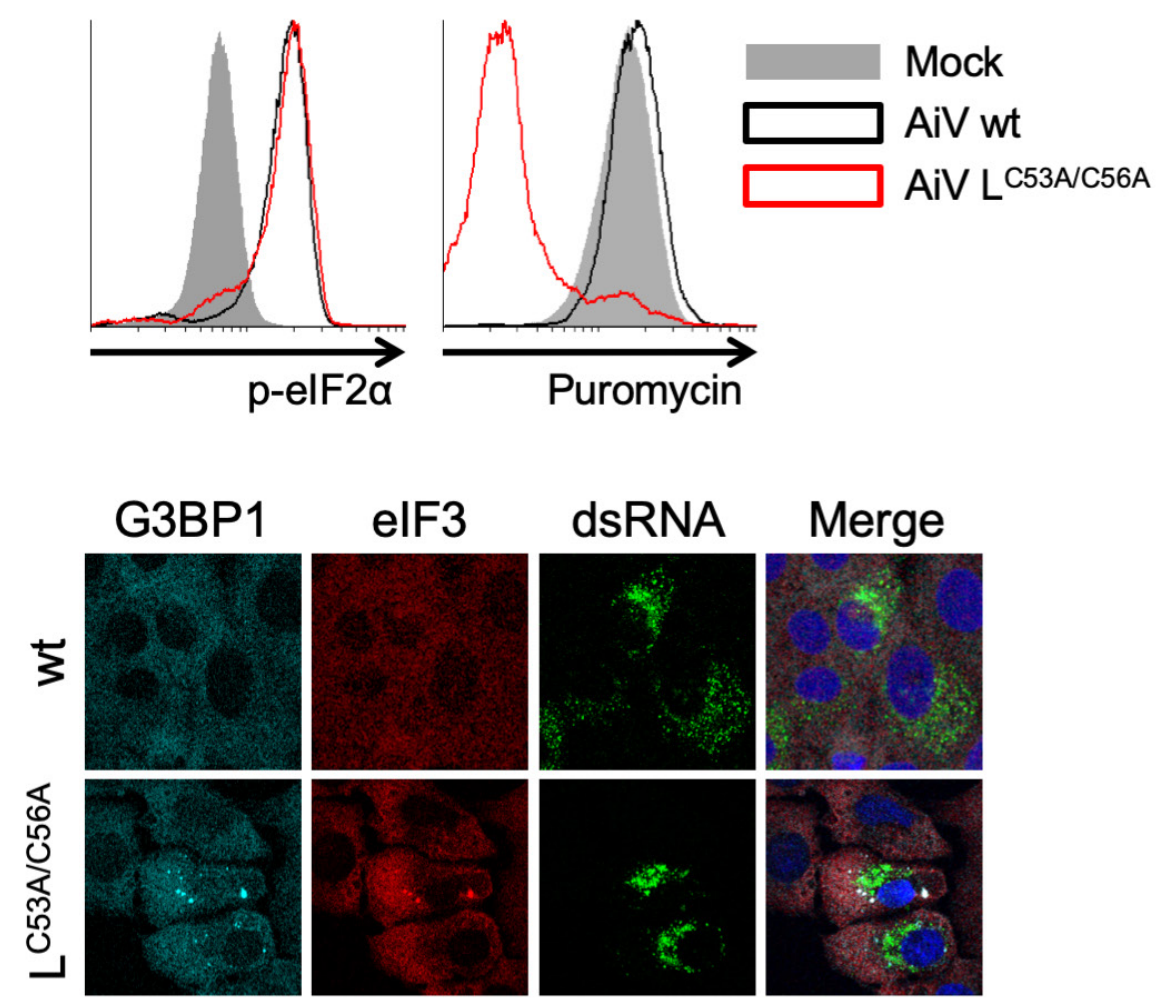

+ Arsenite

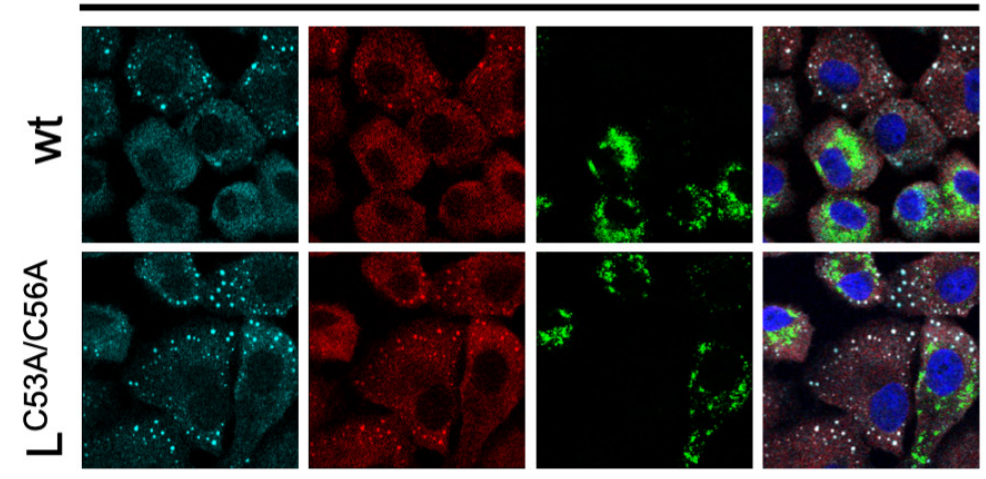

Figure 4: AiVL is the main antagonist encoded by aichivirus. (A) Vero cells were infected with the indicated recombinant aichiviruses at an $\mathrm{MOl}$ of 10 for $8 \mathrm{hrs}$. Translation was labeled with $20 \mathrm{\mu g} / \mathrm{ml}$ puromycin during the final $15 \mathrm{~min}$ of the experiment. Puromycin incorporation, and p-elF2 $\alpha$ content were measured by flow cytometry. (B) Vero cells were infected with the indicated recombinant aichiviruses at an MOl of 1 for 8 hrs. Cells were either left untreated (top panels), or treated with $1.5 \mathrm{mM}$ arsenite during the final hour of the experiment. dsRNA content and the presence of SCs were assessed by IFA using antibodies directed against dsRNA and $\mathrm{C}_{3} \mathrm{BP}$, respectively. 
AiVL is the main antagonist encoded by Aichivirus. To corroborate our findings that AiVL functions as ISR antagonist rescuing translation in the presence of p-eIF2 $\alpha$, and to show that it functions in the context of AiV infection, we created a recombinant AiV in which the $\mathrm{L}$ protein was inactivated by two point mutations (C53A \& $\mathrm{C} 56 \mathrm{~A}$ ). Vero cells were infected with wt AiV or AiV-L-C53A/C56A, and analyzed by flow cytometry for p-eIF2 $\alpha$ levels and active translation rates (Fig 4A), or by IFA for dsRNA content and SG formation (Fig 4B), at 8 hrs pi. Cells infected with AiV or AiV-L-C53A/C56A contained comparable quantities of dsRNA and similar levels of p-eIF2 $\alpha$, indicating efficient virus replication and ISR activation. However, cells infected with AiV-L-C53A/C56A -but not with wt virusdisplayed impaired translation rates and contained SGs. These data unambiguously show that AiVL is an ISR antagonist that rescues translation in the presence of elevated p-eIF2 $\alpha$ levels in AiV-infected cells.

AiVL interacts with translation initiation factor eIF2B. To determine how AiVL rescues translation, we identified its cellular interaction partners by mass spectrometry analysis. HEK293T cells were transiently transfected with plasmids encoding tandem affinity purification (TAP) tagged AiVL. AiVL and its cellular interaction partners, were purified as described previously (8) and analyzed by LC-MS/MS. Two out of five eIF2B subunits (eIF2B $\gamma$ and eIF2B $)$ were modestly but significantly enriched in AiVL pull-down samples relative to empty vector control samples (Fig $5 \mathrm{~A}$ ). To confirm the interaction with eIF2B, AiVL purification samples were analyzed by Western blot using an antibody directed against eIF2B $\alpha$. Indeed, eIF2 $\mathrm{B} \alpha$ co-purified with AiVL, albeit to a much lesser extent than when using Beluga whale-CoV AcP10, which was included as positive control (Fig 5B). Importantly, no interaction was found between the phenotypically inactive mutant AiVL C56A and eIF2B.

> Figure 5: AiVL interacts with translation initiation factor elF2B. (A) Vulcano plot showing the proteins differentially co-immunoprecipitated with TAP-AiVL pulldown compared to empty vector control. Significantly enriched proteins as determined by a t-test ( $5 \%$ permutation-based FDR) are indicated in shades of red that indicate the values set for so. SAINT scores for each of the enriched proteins are indicated by the blue colored labels. (B) The indicated TAP-tagged proteins were transiently overexpressed in HEK293T cells. These proteins were subsequently purified as described previously (8). The final wash step was performed either in low-salt buffer to keep the elF2.elF2B interaction intact (left panels), or in high salt buffer ( $500 \mathrm{mM} \mathrm{KCl)}$ to disrupt elF2.elF2B interactions (right panels). For each sample, the beadassociated protein fraction (indicated as " $\mathrm{B}$ "), and the supernatant fraction (indicated as " $\mathrm{S}$ ") is shown. The presence of co-purified elF2 and elF2B was analyzed by Western blotting using antibodies directed against their respective

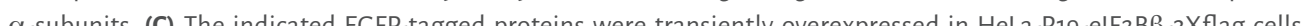
Subseques. Subsequently, elF B complexes were purifed from cell lysates as described previously (8). The washes were performed the same way as in (B), and again the bead-associated protein fraction (indicated as " $\mathrm{B}$ ), and the supernatant fraction (indicated as " $S$ ") are shown for each sample. The presence of co-purified elF2 (represented by its $\alpha$-subunit) and AcP10/ AiVL (assessed using an antibody directed against ECFP) was analyzed by Western blotting.

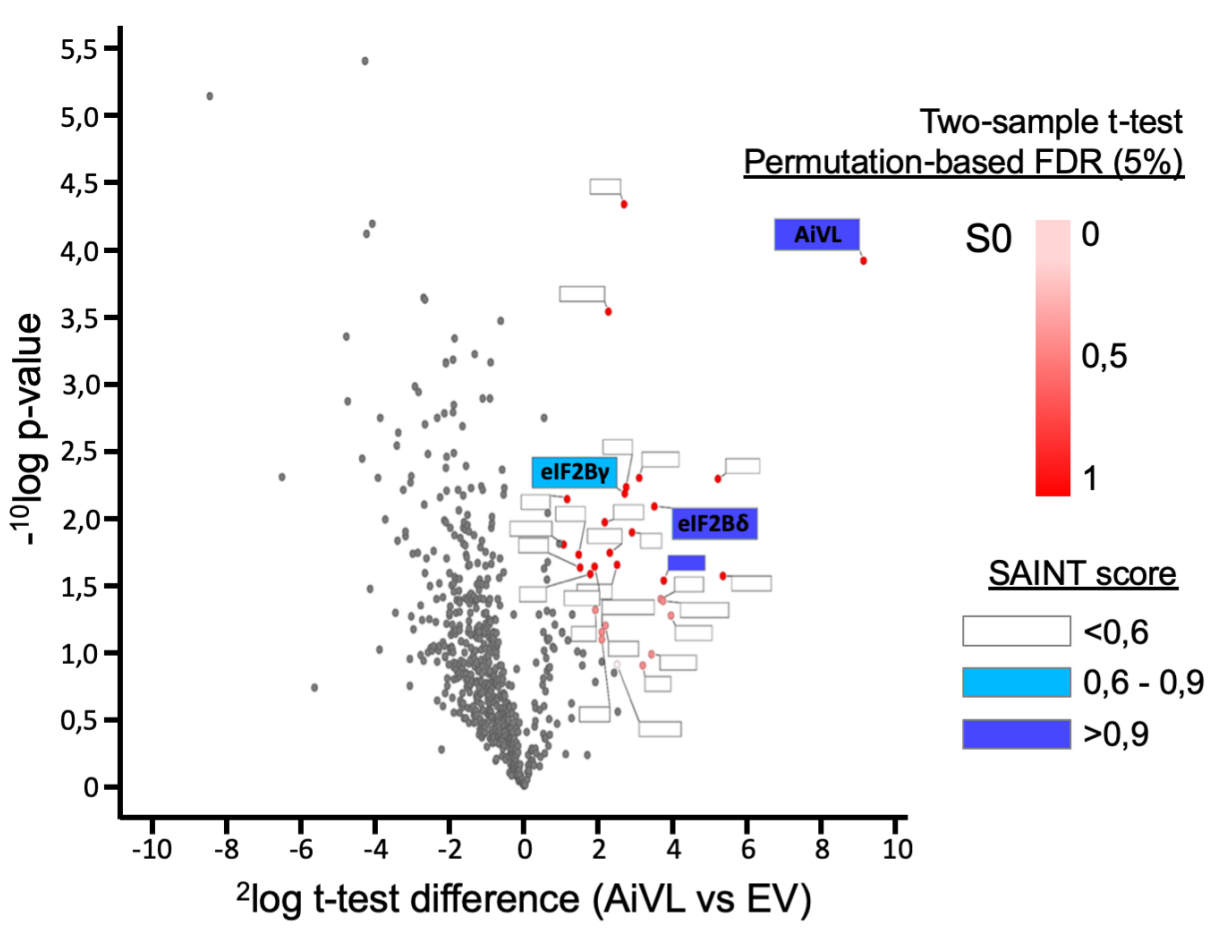

IP: AiVL/AcP10

Low Salt Wash High Salt Wash

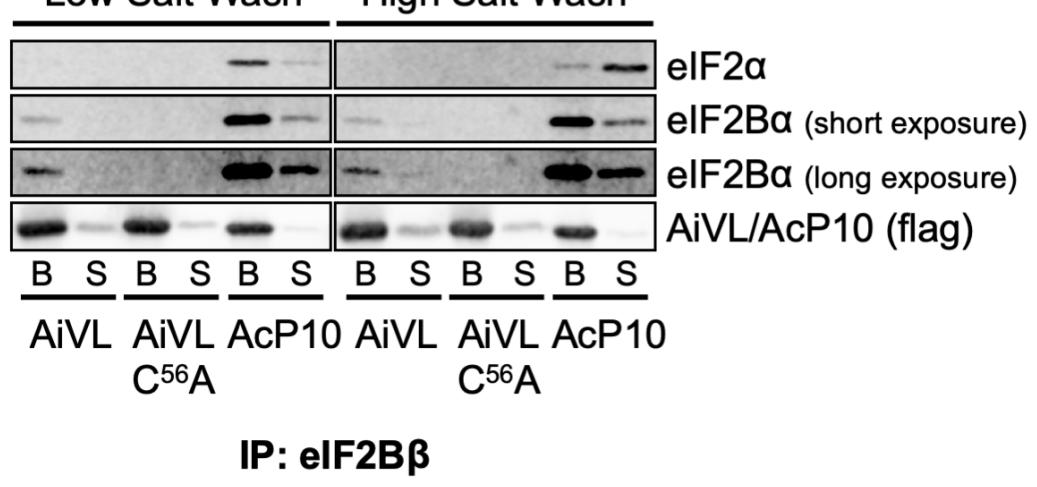

Low Salt Wash High Salt Wash

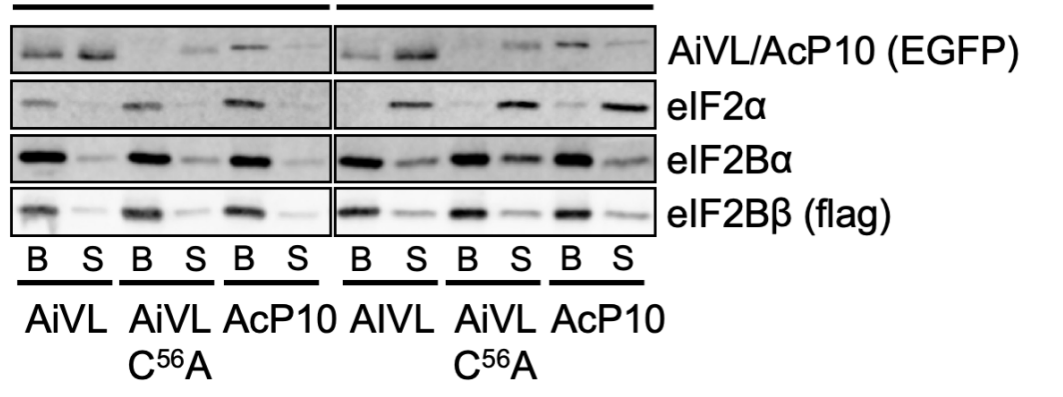


As reported, purification of AcP1o also indirectly co-purifies eIF2 $\alpha$, since the eIF2 • eIF2B macrocomplex remains intact during this purification procedure $(8,18)$. No eIF2 $\alpha$ band was observed in the AiVL pulldown sample, but given the low co-IP efficiency when using AiVL as bait, it is to be expected that the eIF2 $\alpha$ signal may fall below the detection limit even if eIF2 $\alpha$ is in fact present (Fig $5 B$ ). To address the possibility that AiVL may interact with eIF2 rather than eIF2B, we disrupted the eIF2•eIF2B interaction using a high-salt wash (500 $\mu \mathrm{M} \mathrm{KCl)} \mathrm{(Fig} \mathrm{5B,} \mathrm{right} \mathrm{panels),} \mathrm{and} \mathrm{found} \mathrm{that} \mathrm{eIF2B} \mathrm{still} \mathrm{associates} \mathrm{with} \mathrm{AiVL}$ under these conditions. This demonstrates that AiVL directly binds eIF2B, independent of the presence of eIF2.

As a complimentary approach, we pulled down eIF2B from HeLa cells stably expressing flag-tagged eIF2B $\beta$ (HeLa-eIF2B $\beta$-3Xflag, (19)), and analyzed co-purified eIF2 $\alpha$ and AiVL by Western blot (Fig $5 \mathrm{C}$ ). AiVL, like AcP10, could be detected in eIF2B $\beta$ pulldown samples, again suggesting that AiVL interacts with eIF2B. A high-salt wash step that disturbs the eIF2•eIF2B interaction, released most of the eIF2 into the supernatant fraction, but had only a minor impact on the association of AiVL (Fig 5 C, compare lanes 1 and 2 with lanes 7 and 8). Taken together, the data suggest that AiVL rescues translation in presence of p-eIF2 through its binding to eIF2B. The amount of eIF2B $\alpha$ co-purified with AiVL is consistently low (Fig $5 \mathrm{~B}$ ), while the reverse IP using eIF2B as bait efficiently yields AiVL (Fig $5 \mathrm{C}$ ). This is likely caused by a vast excess of AiVL protein over eIF2B complexes upon transient overexpression, which causes most AiVL to be free of eIF2B. Additionally, and unlike the AcP10•eIF2B interaction, the AiVL•eIF2B association may be unstable, and less likely to survive the lengthy TAP-AIVL pulldown procedure $(\sim 20 \mathrm{hrs})$ than the shorter eIF2B-flag pulldown procedure $(\sim 3 \mathrm{hrs})$. A relatively unstable AiVL•eIF2B interaction can also be deduced from the observation that incubation of AiVL.eIF2B complexes in wash buffer for 15 min released approximately half of the AiVL protein from eIF2B into the supernatant fraction (Fig $5 \mathrm{C}$, lanes 1 and 2).

AiVL counteracts the association of p-eIF2 to eIF2B. AcP1o binding to eIF2B prohibits the inhibition of eIF2B GEF function through competitive displacement of p-eIF2 (8). To test the effects of AIVL on the formation of p-eIF2•eIF2B complexes, we transfected HeLa-eIF2B $\beta$-3Xflag cells with plasmids encoding AiVL or control proteins, and treated them with arsenite to induce eIF2 phosphorylation. We subsequently pulled down eIF2B from cell lysates and analyzed the amount of co-immunoprecipitated p-eIF2 $\alpha$ by Western blotting. AcP1o expression interfered with p-eIF2•eIF2B complex formation, resulting in a significant reduction in the amount of p-eIF2 $\alpha$ bound to eIF2B ((8), Fig 6A). AiVL expression has a similar effect, suggesting that AiVL -like AcP1O- keeps eIF2B active despite eIF2 phosphorylation by counteracting p-eIF2•eIF2B complex formation. The remaining p-eIF2 $\alpha$ signal (approximately $27 \%$ of the control sample) corresponds to the background

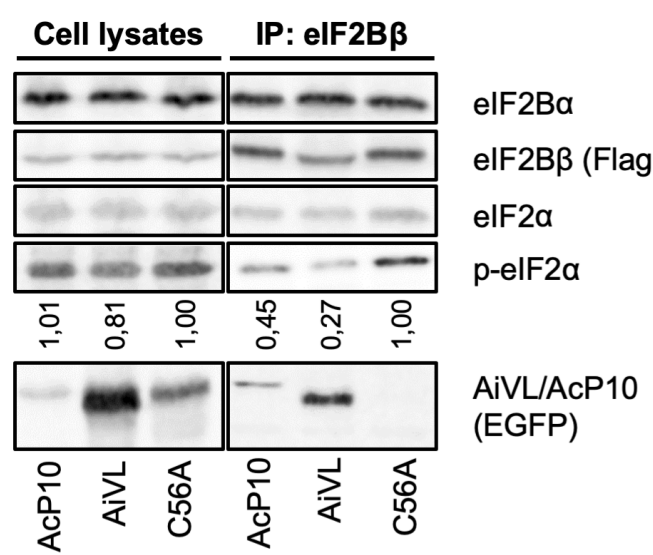

Cell lysates
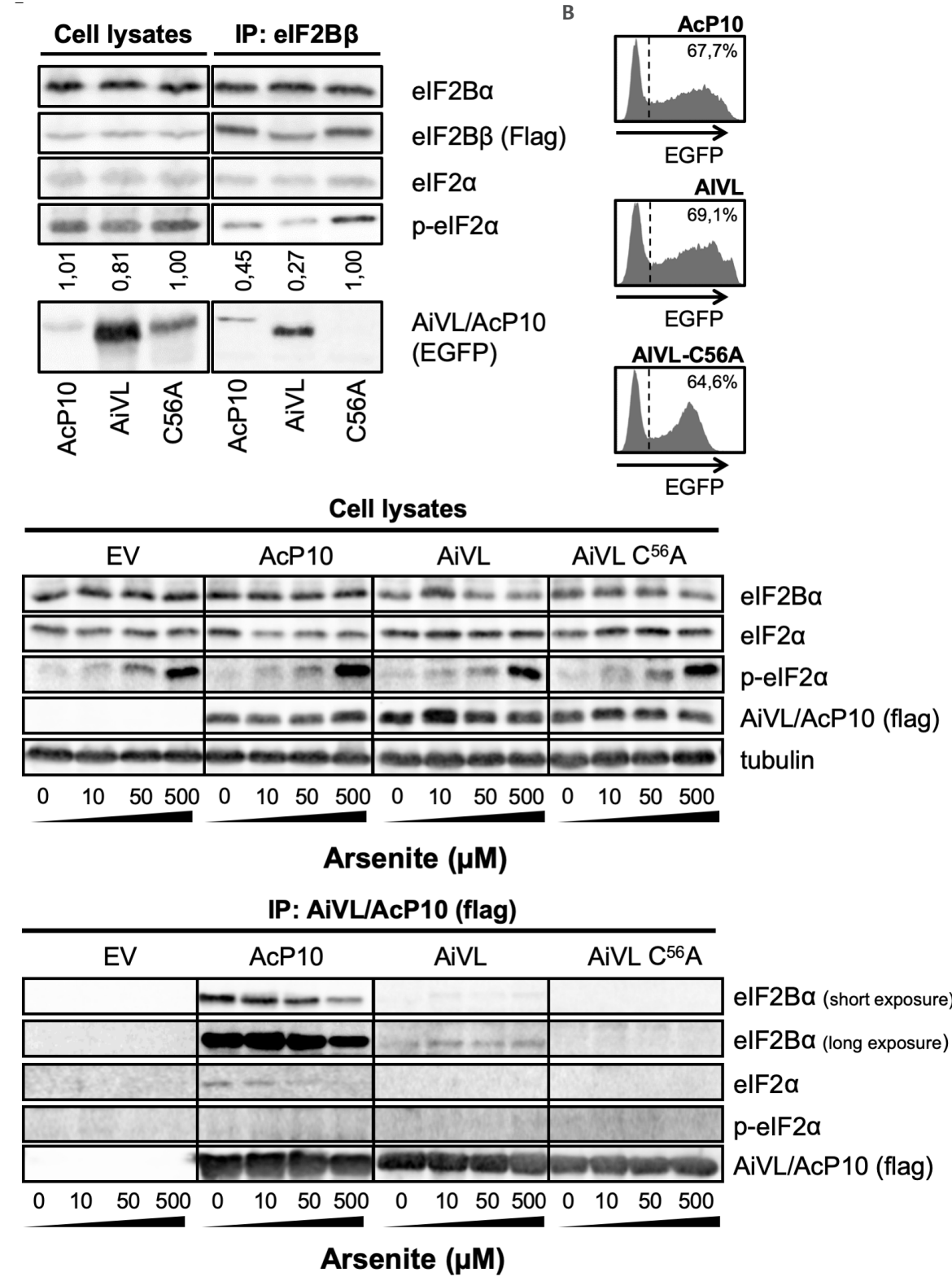

Figure 6: AiVL counteracts the association of $\mathrm{p}$-elF2 to elF2B. The indicated ECFP-tagged proteins were transiently overexpressedin Hela-R19-elF2B $\beta$-3Xflag cells Cellswere treated with 250 M Marsenite for 1 hrtoinduce phosphorylation of elF2 $\alpha$. (A) elF2B complexes were purified from cell lysates as described previously (8). The presence of co-puried $\mathrm{elF} 2$, $\mathrm{p}$-elF2, and $\mathrm{ACP} 10 /$ ECFP, respectively. (B) Flow cytometry analysis of the transfection efficiencies obtained in the experiment shown in (A) (C) HEK293T cells were transiently transfected with plasmids encoding the indicated TAP-tagged proteins. Cells were subsequently treated with the indicated arsenite concentrations for $1 \mathrm{hr}$, and the overexpressed proteins were purified from cell lysates. The presence of co-purified elF2, p-elF2, and elF2B was assessed by Western blotting using antibodies directed against elF $2 \alpha, p$-elf $2 \alpha$, and elF $2 B \alpha$, respectively. 
level that is expected at the transfection efficiencies of $\sim 70 \%$ obtained in this experiment (Fig 6B). Our data thus suggest that the AiVL•eIF2B interaction prevents p-eIF2 binding to eIF2B. This effect is p-eIF2-specific, since the total amount of eIF2 bound to eIF2B was unaffected by the presence of AiVL.

Next, we overexpressed TAP-tagged AiVL or control proteins in HEK293T cells that were subsequently treated with several arsenite concentrations to induce increasing levels of p-eIF2. We then tandem affinity purified the overexpressed proteins and assessed co-purified eIF2B and (p-)eIF2, using antibodies directed against eIF2B $\alpha$, eIF2 $\alpha$ and p-eIF2 $\alpha$ (Fig $6 \mathrm{C}$ ). In line with previous data (8), the efficiency of eIF2B co-purification with AcP1o is lower when cells were treated with arsenite to induce eIF2 phosphorylation, indicative of a competition between AcP10 and p-eIF2 for eIF2B binding. In contrast, AiVL•eIF2B complexes, while present in relatively low quantities, were not affected by p-eIF2 concentrations. Importantly, neither AcP10•eIF2B, nor AiVL•eIF2B complexes contained detectable amounts of p-eIF2, again indicating that AiVL counteracts the p-eIF2•eIF2B interaction. Collectively, our data indicate that AiVL has a similar effect as AcP10, rendering eIF2B GEF function insensitive to inhibition by p-eIF2, but some aspects of AiVL's interaction with eIF2B are remarkably different from those of AcP1o. First, the amount of eIF2B co-purified with AiVL is consistently and significantly lower than with AcP10 (Fig 5B and 7). Second, AiVL's interaction with eIF2B seems relatively unstable (Fig $5 C$, lanes 1 and 2). Third, the AiVL•eIF2B interaction is insensitive to high levels of p-eIF2 (Fig 6). Fourth, AiVL is a highly soluble protein, in stark contrast to AcP10, which was shown to largely aggregate in insoluble perinuclear deposits upon overexpression (8).

> Figure 7: Mouse KoVL, but not the KoVLs from other species, also acts as ISR antagonist. (A) phylogenetic tree of kobuvirus $L$ proteins. The scale bar represents the number of substitutions per site. (B) Analysis of the ISR antagonist function of the indicated kobuvirus $L$ proteins in HeLa cells by IFA. Shown is the percentage of cells that contain SGs after treatment with $250 \mu \mathrm{M}$ arsenite for $1 \mathrm{hr}$, within the population of transfected cells (ECFP positive, shown in green), as well as untransfected cells from the same wells (ECFP negative, shown in grey). (C) HeLa cells were transiently transfected with plasmids encoding the indicated ECFP tagged proteins and subsequently analyzed by flowcytometry as in Fig $2 \mathrm{D}$ and $3 \mathrm{C}$. Plotted is the ratio in mean fluorescence intensity (MFI) values of the puromycin staining, between the $\mathrm{p}$-elF $2 \alpha^{\text {high }}$ (stressed) and $\mathrm{p}$-elF $2 \alpha^{\text {low }}$ (unstressed) cell populations. A ratio of 1 (indicated by the dotted line) indicates

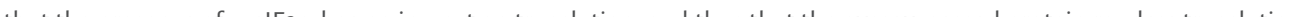
insensitive to $p$-elf. (D) HeLa cells transiently overexpressing the indicated ECP tagged proteins were treated with 250 $\mu \mathrm{M}$ arsenite for $1 \mathrm{hr}$, and translation was labeled using puromycin during the final $15 \mathrm{~min}$ of the experiment. Puromycin incorporation was analyzed by flowcytometry. Plotted is the ratio in mean fluorescence intensity (MFI) values between the ECFPhigh (transfected) and ECFPlow (untransfected) cell populations. A ratio of 1 (indicated by the dotted line) indicates that the overexpressed protein has no impact on translation in stressed cells. Values higher than 1 indicate that the overexpressed protein rescues translation in arsenite-treated cells. (E) The indicated TAP-tagged proteins were overexpressed in HEK293T cells and purified as described above. The presence of co-purified elF2B was analyzed by Western blotting using antibodies directed against Weste blo in assessed by IFA and plotted as in (B)

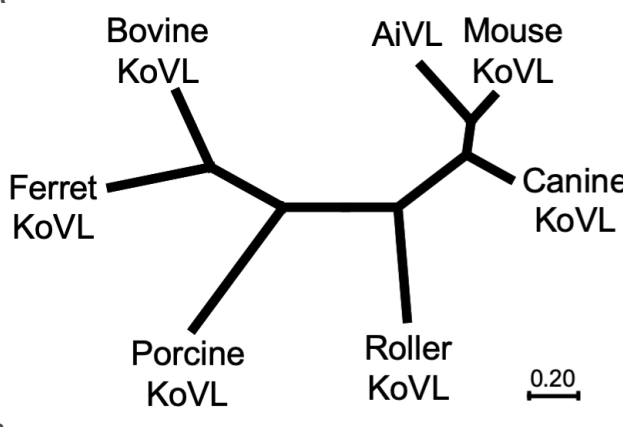

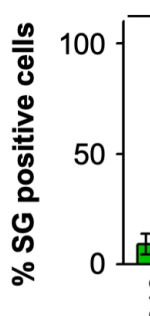

+ Arsenite
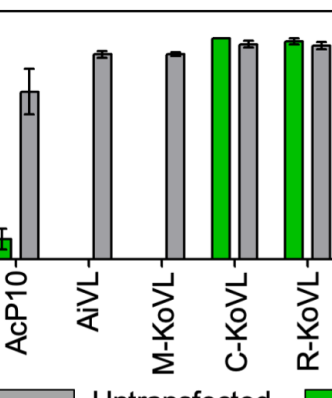

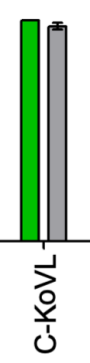

Untransfected

年

Total cell lysates
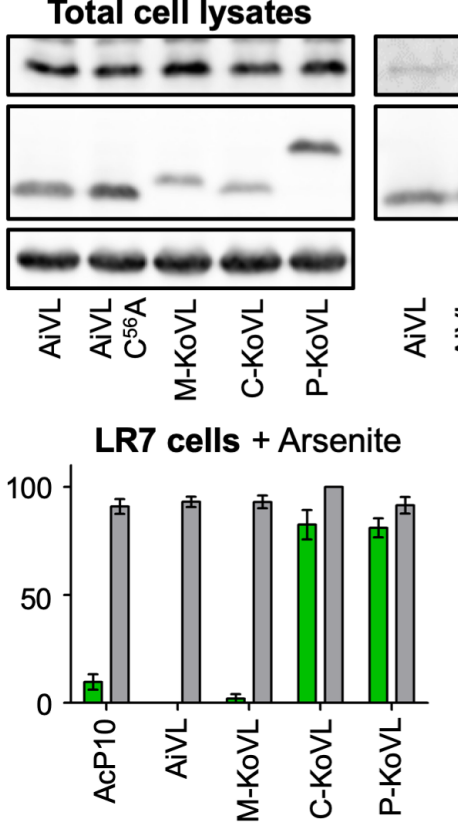

LR7 cells + Arsenit

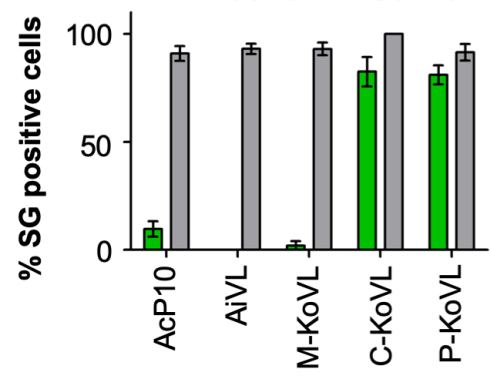

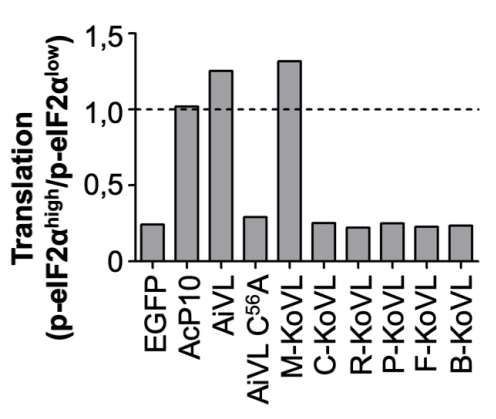

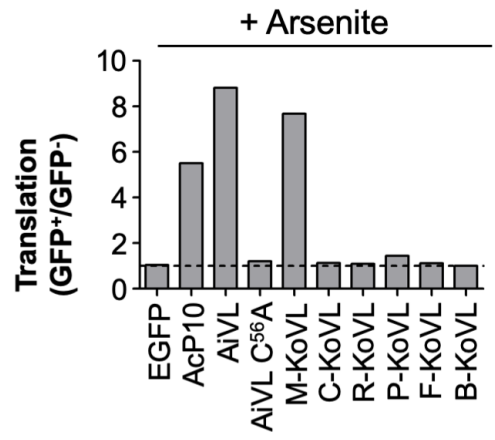

IP: KoVL

KoVL proteins (Flag)

Tubulin

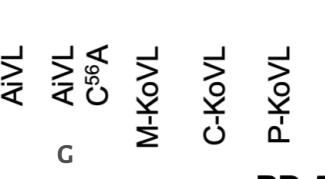

PD-5 cells + Arsenite

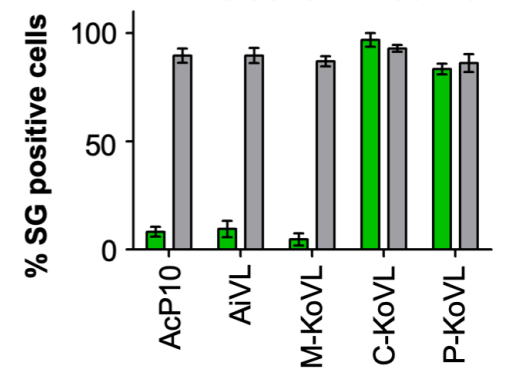


Mouse KoVL, but not the KoVLs from more distantly related species, can also antagonize the ISR. Besides AiVL, the kobuvirus family consists of viruses that infect mammalian species such as mice (Mouse KoV), dogs (Canine KoV), pigs, (Porcine KoV), and cattle (Bovine KoV). Each of these viruses contains an L protein homologous to AiVL (Fig 7A), with amino acid identities ranging from $\sim 55 \%$ to $\sim 80 \%$. To test whether the ISR inhibiting function of AiVL is conserved for $\mathrm{L}$ proteins throughout the Kobuvirus genus, we separately overexpressed the $\mathrm{L}$ proteins of AiV, Mouse KoV (M-KoVL), Canine KoV (C-KoVL), Roller KoV (R-KoVL), Bovine KoV (B-KoVL), Ferret KoV (F-KoVL), and Porcine KoV (P-KoVL) in HeLa cells and tested their ability to counteract arsenite-induced SG formation.

Surprisingly, only Mouse KoV-L - which is phylogenetically most related to AiVL- was able to prevent SG formation (Fig 7B). These data were corroborated by FACS experiments showing that AiVL or M-KoVL expressing cells display unimpaired translation rates irrespective of the phosphorylation status of eIF2, either upon DNA transfection alone (Fig 7C), or after arsenite treatment (Fig 7D). Furthermore, in a KoVL co-IP experiment we show that only AiVL and M-KoVL detectably interact with eIF2B (Fig $7 \mathrm{E}$ ). Consistently, the four residues that may form the $\mathrm{Zn}$-finger structure $\left(\mathrm{H}_{2} \mathrm{O}, \mathrm{H}_{24}, \mathrm{C}_{53}\right.$, and $\mathrm{C}_{56}$ ) and are essential for AiVL's ISR antagonist function (Figs 3 and S1), are strictly conserved in AiVL and M-KoVL, whereas the other KoVLs lack residues homologous to $\mathrm{H} 2 \mathrm{O}$ and $\mathrm{H} 24$ in AiVL. Despite high conservation of the eIF2B subunits between mammalian species, we could not exclude the possibility that the KoVL proteins interact specifically with eIF2B from their respective host species. Therefore, we tested ISR antagonism by a selection of KoVL proteins (AiVL, M-KoVL, C-KoVL, and $\mathrm{P}-\mathrm{KoVL}$ ) in cell lines of mouse (LR7) and pig (PD-5) origin, using transient overexpression and subsequent analysis of arsenite-induced SG formation. In both LR7 cells (Fig 7F) and PD-5 cells (Fig 7G), only AiVL and M-KoVL are functional ISR antagonists, demonstrating that host species specificities do not underlie the differences in ISR antagonism between KoVL proteins. Rather, these data suggest that besides AiVL and M-KoVL none of the KoVLs function as ISR antagonist. Thus, the acquisition of AiVL's ISR antagonist function was likely a relatively recent evolutionary event that occurred within the Aichi A lineage.

\section{Discussion}

We set out to investigate how kobuviruses, and AiV in particular, counteract antiviral ISR activity. We determine that AiV encodes a highly potent ISR antagonist (AiVL) that prevents cellular translational arrest in the presence of $\mathrm{p}$-eIF2. AiVL binds translation initiation factor eIF2B, and thereby specifically prevents subsequent association of p-eIF2. Consequently, in the presence of AiVL, eIF2B preferentially binds its substrate (non-phosphorylated eIF2) over its inhibitor (phosphorylated eIF2), and thus remains active even under conditions of stress. This mode of action is similar to that of the recently described beluga whale-CoV protein AcP1o, and differs from that of all other known ISR antagonists. AcP1o and AiVL are prototypes of a new Class of ISR antagonist, that targets the ISR downstream of eIF2 phosphorylation, at the level of eIF2B GEF function inhibition. ISR inhibition by precluding the p-eIF2•eIF2B interaction thus appeared at least twice, in phylogenetically distinct virus families, through convergent evolution.

AiVL and AcP1o both bind eIF2B, and correspondingly, they localize to the cytoplasm. However, AiVL has a diffuse localization, whereas AcP1o is largely aggregated in insoluble perinuclear deposits. Consequently, only low AcPio concentrations remain soluble and can participate in competition with p-eIF2 for eIF2B binding (8), while soluble AiVL levels may vastly exceed those of p-eIF2 or eIF2B (Fig S2). Furthermore, in contrast to AcP10, AiVL has a low stability interaction with eIF2B, purification of AiVL yields little co-purified eIF2B, and AiVL was not detectably displaced from eIF2B by high p-eIF2 levels. These differences between AiVL and AcPio may be related to their difference in solubility. First, since soluble AiVL is present in vast excess over eIF2B, most AiVL will not be bound to eIF2B and thus eIF2B co-IP is expected to be inefficient when using AiVL as bait (Figs 5 and 6). AcPio on the other hand is largely insoluble and thus the levels of soluble AcPio available for eIF2B binding remain relatively low. Second, increasing p-eIF2 concentrations might be unable to outcompete AiVL due to a large excess of soluble AiVL over p-eIF2, even under severe stress conditions (Fig 6). Third, given such high levels of AiVL, a relatively low stability eIF2B interaction would suffice to prevent inhibition of eIF2B activity (Fig 5).

ISR antagonists can be classified in several categories based on which signal transduction event in the ISR pathway they counteract. Most ISR antagonists are specific to a particular eIF2 $\alpha$ kinase, preventing either its activation (class 1 antagonist) or its kinase activity on eIF2 $\alpha$ (class 2 antagonist). However, virus infection often causes extensive changes in the intracellular environment and may activate more than one eIF2 kinase. Thus, these antagonists may fail to prevent ISR activity entirely. Alternatively, an antagonist may induce dephosphorylation of p-eIF2 (class 3 antagonist), which counters the ISR irrespective of the kinase involved. However, this approach only mediates a reduction in p-eIF2 levels to some extent based on the cell's capacity for eIF2 dephosphorylation, and thus the ISR may still signal under conditions of severe stress $(20,21)$. AiVL does not belong to any of these classes, and instead renders eIF2B immune to eIF2 phosphorylation. Thereby, it blocks the ISR regardless of the nature or severity of the stress trigger, or the eIF2 kinase(s) involved. Thus, AiVLs mode of action is arguably the most effective way to block the ISR described so far.

RNA viruses typically obtain their genes via recombination events, and thus the chance of acquiring antagonists with a certain mode-of action-depends on the availability of other 
(cellular) RNAs that encode proteins with such functions. For example, dsRNA binding domains are relatively common, so recombination events that lead to the acquisition of dsRNA binding proteins should occur often. In line with this, many viruses encode specific PKR inhibitors that act by sequestering dsRNA $(17,22-25)$. In contrast, no host cell proteins have been described that bind eIF2B and interfere with p-eIF2 binding, and thus a protein with this mode of action is unlikely to be obtained by viruses. In line with this, both viral proteins that prevent p-eIF2 from inhibiting eIF2B, AcP1O and AiVL, seem to have obtained their ISR antagonist function via gain-of-function substitutions in pre-existing viral proteins, rather than via recombination with RNAs encoding eIF2B-binding proteins. Our data indicate that most $\mathrm{L}$ proteins encoded by related viruses in the KoV family do not function as ISR antagonists. Thus, it is highly likely that the ancestral KoVL protein was not an ISR inhibitor, and that this function was obtained relatively recently within the AiV A lineage. Remarkably, since we also demonstrate that Aichivirus has no ISR antagonists besides AiVL, the other KoVs must either lack ISR antagonists altogether, or encode an antagonist(s) that is absent in AiV.

The ISR plays a role in a variety of different cell biological processes, both during normal cell function and under conditions of stress (26-29). Its activity is typically balanced and proportional to the situation at hand, and deviations from this balance, both towards a hypoactive or hyperactive ISR, may have pathological consequences (30-32). Most notoriously, a variety of neurodegenerative diseases have been linked to an overactive ISR, including but not limited to Parkinson's disease, Alzheimer's disease, Vanishing White Matter, and prion disease (33-37). A causal connection was even established between neuropathology and an overactive ISR by showing that ISR inhibitors may alleviate neurotoxicity $(28,33,36,38-40)$. Viruses - through their rapid rates of evolution over extensive periods of time, and under strong selective pressure to countermeasure the ISR - could teach us the most effective ways to interfere with ISR signaling. The identification of novel types of ISR antagonists like AiVL and AcPio and elucidation of the details of their mode of action, may therefore provide the basis for novel strategies to modulate the ISR for therapeutic purposes.

\section{Acknowledgments}

The work was supported by the Netherlands Organization for Scientific Research through a Vici grant (NWO-918.12.628) to FJMvK, a Veni grant (NWO-863.13.008) to MAL and a NWO graduate programme grant (NWO-022.004.018) to LJV. We thank Prof. David Ron for kindly providing HeLa cells stably expressing $3 X$ flag-tagged eIF2B $\beta$, dr. Alex Greninger for the pAV aichivirus cDNA clone, dr. George Belov for HeLa-R19 cells, and prof. Jerry Pelletier for translation inhibitor Pateamine A.

\section{Materials \& Methods}

Chemical compounds. ISRIB (SML0843) and puromycin (P9620) were obtained from SigmaAldrich and used at a final concentration of $200 \mathrm{nM}$ and $20 \mu \mathrm{g} / \mathrm{mL}$, respectively. Sodium arsenite was purchased at Riedel-de-Haën (UN1686).

Cells and Viruses. HEK293T, HeLa-R19, and Vero cells were maintained in DMEM (Lonza) supplemented with $10 \%$ FCS and penicillin-streptomycin (100 units $/ \mathrm{mL}$ and $100 \mu \mathrm{g} / \mathrm{mL}$ ). To create recombinant AiV- $\mathrm{C}^{53} \mathrm{~A} / \mathrm{C}^{56} \mathrm{~A}$, the double mutation was introduced in the AiV infectious clone pAV (41) by site-directed mutagenesis. The infectious clones were linearized by HindIII digestion and infectious RNA was synthesized by a T7 in vitro transcription reaction (Promega). Infectious RNA was transfected in Vero cells using Lipofectamine 2000 reagents (Invitrogen), and AiV stocks (encoding either wt AiVL or AiVL $\mathrm{C}^{53} \mathrm{~A} / \mathrm{C}^{56} \mathrm{~A}$ ) were harvested three days post transfection. Integrity of the AiVL gene was confirmed by sanger sequencing, and virus titers were determined using end-point dilution assays on Vero cells.

Immunofluorescence Assay. Immunofluorescence assays were performed as described previously (17), using primary antibodies rabbit- $\alpha-\mathrm{G}_{3} \mathrm{BP2}$ (A302-040A, 1:200; Bethyl Laboratories) mouse- $\alpha$-dsRNA J2 (1:1000; English Scientific \& Consulting), rabbit- $\alpha-G_{3} B P 1$ (ARP37713-T100, 1:200; Aviva), goat- $\alpha$-eIF3ך (sc-16377, 1:200; SantaCruz) and secondary antibodies donkey- $\alpha$-mouse-Alexa488 (A-21202, 1:200; Thermo Fisher Scientific), donkey- $\alpha$ goat-Alexa568 (A-11058, 1:200; Thermo Fisher Scientific), donkey- $\alpha$-rabbit-Alexa647 (A-31573, 1:200; Thermo Fisher Scientific).

Western Blot Analysis. Western blot assays were performed as described previously (17), using primary antibodies rabbit- $\alpha$-eIF2 $\alpha$ (9722, 1:1000; Cell Signaling), rabbit- $\alpha$-p-eIF2 $\alpha$ (ab32157, 1:1000; Abcam), mouse- $\alpha$-tubulin (T9026, 1:2000; Sigma-Aldrich), rabbit- $\alpha$-eIF2B $\alpha$ (18010-1-AP, 1:1000; Protein Tech), mouse- $\alpha$-Flag (F3165, 1:1000; Sigma-Aldrich), or rabbit- $\alpha$ EGFP (42), and secondary antibodies goat- $\alpha$-mouse-IRDye680 (926-68070, 1:15,000; LI-COR) or goat- $\alpha$-rabbit-IRDye800 (926-32211, 1:15,000; LI-COR).

Flow Cytometry Analysis of eIF2 $\alpha$ Phosphorylation. Cells were released using trypsin and fixed with paraformaldehyde ( $4 \%$ in PBS) for 20 min. Cells were washed with FACS buffer (PBS $+1 \%$ BSA) and subsequently incubated for $10 \mathrm{~min}$ in ice-cold methanol. Cells were washed once with FACS buffer and incubated with primary antibodies rabbit- $\alpha$-p-eIF2 $\alpha$ (ab32157, 1:100; Abcam) and mouse- $\alpha$-puromycin (MABE343, 1:100; Merck Millipore) for 45 min. After 3 wash steps in FACS buffer, cells were incubated in secondary antibodies donkey$\alpha$-mouse-cy5 (715-175-150, 1:200; Jackson ImmunoResearch) and goat- $\alpha$-rabbit-Alexa594 (A11012, 1:200; Invitrogen) for $45 \mathrm{~min}$ at room temperature in FACS buffer. Cells were washed 
twice, resuspended in PBS $+1 \%$ paraformaldehyde and analyzed on the FACSCanto II (BD Biosciences).

AP-MS. Protein samples were separated by SDS-PAGE, and stained with Coomassie colloidal blue (Bio-Rad). After a short run, the entire sample between running fronts was sliced out and reduced, alkylated, and digested as previously described (43). Digests were subjected to nLC-MS/MS analysis using an Agilent 1290 Infinity UHPLC system (Agilent) coupled to an Orbitrap Q Exactive Plus mass spectrometer (Thermo Scientific). Dried peptides were reconstituted in 10\% formic acid (FA) and delivered to a trap column (Dr Maisch Reprosil $\mathrm{C} 18,3 \mu \mathrm{m}, 2 \mathrm{~cm} \times 100 \mu \mathrm{m})$ at $5 \mathrm{\mu l} / \mathrm{min}$ with solvent $\mathrm{A}(0.1 \% \mathrm{FA}$ in water). Next, peptides were chromatographically separated onto an analytical column (Agilent Poroshell EC-C18, $2.7 \mu \mathrm{m}$, $50 \mathrm{~cm} \times 75 \mu \mathrm{m})$ at $300 \mathrm{~nL} / \mathrm{min}$, as previously described (44). The gradient was as follows: $13-$ $40 \%$ solvent B (0.1\% FA in $80 \%$ Acetonitrile) in $65 \mathrm{~min}$, to $100 \%$ solvent B in $3 \mathrm{~min}, 1 \mathrm{~min}$ of $100 \%$ solvent $B$, and finally equilibration of the chromatographic columns with $100 \%$ solvent $A$ for the following $10 \mathrm{~min}$ before injection of the next sample. Total analysis time was $90 \mathrm{~min}$. The eluent was sprayed via distal coated emitter tips butt-connected to the analytical column. The mass spectrometer was operated in data-dependent mode, automatically switching between MS and MS/MS. Full-scan MS spectra (from m/z 375 to 1600) were acquired in the Orbitrap with a resolution of 35,000 at $\mathrm{m} / \mathrm{z} 400$. The 10 most intense ions within the survey scan were selected for HCD fragmentation with normalized collision energy set to $25 \%$. The MS/MS AGC target value was set to $5 e 4$ with a maximum ion injection time of $120 \mathrm{~ms}$. Dynamic exclusion was set to $12 \mathrm{~s}$. Each raw data file recorded by the mass spectrometer was processed and quantified with Proteome Discoverer (version 2.1, Thermo Scientific), and searched against a Swissprot human database (version 2016 03, 20,199 sequences) supplemented with the Aichivirus polyprotein sequence (Uniprot id AoA2S1JL36) using Mascot software (version 2.5.1, Matrix Science). Trypsin/ $\mathrm{P}$ was chosen as the protease, cysteine carbamidomethylation was selected as fixed modification, and oxidation of methionine as variable modifications. Precursor and fragments mass tolerance were set to $20 \mathrm{ppm}$ and $0.05 \mathrm{Da}$, respectively. Proteins were further filtered for contaminants and highly abundant proteins (such as keratins, tubulins, and ribosomal proteins), number of unique peptides $(>0)$, and number of peptides (>1). All peptide-spectrum matches (PSM) were validated with $5 \%$ false discovery rate using Percolator (45). Only PSMs with a minimum length of 6 amino acids were kept. To discriminate bona fide protein interactors of AiVL from the background, we further analyzed the hits by SAINT scoring (46).

Immunoprecipitation of flag-tagged eIF2B $\beta$. HeLa-R19-eIF2B $\beta$-3Xflag were transfected with plasmids encoding the indicated EGFP-tagged proteins using Lipofectamine 2000 transfection reagent according to the manufacturer's protocol. Transfection medium was replaced by fresh medium $24 \mathrm{hrs}$ post transfection, and cells were allowed to express the protein of interest for a total of 48 hours. For each sample, $3.0^{*} 10^{7}$ cells were lysed in $500 \mathrm{ul}$ lysis buffer (50 mM Tris- $\mathrm{HCl} \mathrm{pH7.4,} 150 \mathrm{mM} \mathrm{NaCl}$, I mM EDTA, I mM DTT, 10\% glycerol, $1 \%$ Triton X100, protease inhibitors [Roche], phosphatase inhibitors [Sigma-Aldrich]) for $15 \mathrm{~min}$ on ice. After spinning down to remove cell debris, $10 \mu \mathrm{l}$ magnetic anti-Flag Beads (SigmaAldrich) was added to the supernatants and incubated rotating for 2 hours at $4^{\circ} \mathrm{C}$. Beads were then washed four times in wash buffer (50 mM Tris-HCl pH7.4, $150 \mathrm{mM} \mathrm{NaCl}$, I mM EDTA, $1 \mathrm{mM}$ DTT, 10\% glycerol, $1 \%$ Triton X100), and resuspended in 1X LSB. If indicated (See Fig. 5), the third wash step was done for 15 min in standard wash buffer, or high salt wash buffer (50 mM Tris- $\mathrm{HCl}$ pH7.4, $150 \mathrm{mM} \mathrm{NaCl}, 500 \mathrm{mM} \mathrm{KCl}, 1 \mathrm{mM} \mathrm{EDTA}, 1 \mathrm{mM}$ DTT, 10\% glycerol, $1 \%$ Triton X100) to dissociate eIF2 from eIF2B. Samples were then separated by SDS-PAGE and analyzed by Western blotting using the indicated antibodies.

Immunoprecipitation of TAP-tagged AiVL. $1.5^{*} 10^{7} \mathrm{HEK}$ cells were transfected with plasmid encoding the indicated TAP-tagged protein using PEI transfection reagents as described previously (47). $24 \mathrm{hrs}$ post transfection, cells were harvested and lysed in $500 \mu \mathrm{l}$ lysis buffer (50 mM Tris-HCl pH7.4, 150 mM NaCl, 1 mM EDTA, I mM DTT, 10\% glycerol, 1\% Triton X100, protease inhibitors [Roche]). $20 \mathrm{ul} \mathrm{S}$-beads was added to each sample, and incubated rotating for 2 hours at $4^{\circ} \mathrm{C}$. Beads were washed twice in wash buffer $(50 \mathrm{mM}$ Tris- $\mathrm{HCl}$ pH7.4, $150 \mathrm{mM}$ $\mathrm{NaCl}, 1 \mathrm{mM}$ EDTA, $1 \mathrm{mM}$ DTT, $10 \%$ glycerol, $1 \%$ Triton X100), once in $3 \mathrm{C}^{\text {pro }}$ cleavage buffer (HRV $3 \mathrm{C}$ Protease Solution Kit; Thermo Scientific), and resuspended in $250 \mu \mathrm{l} 3 \mathrm{C}^{\text {pro }}$ cleavage buffer. $3 \mathrm{C}^{\text {pro }}$ was then added to proteolytically release proteins from the $\mathrm{S}$-beads rotating overnight at $4^{\circ} \mathrm{C}$. Supernatants were collected and transferred to new tubes. $10 \mu \mathrm{l}$ Strep-Tactin beads (IBA) was added to each sample and incubated rotating for 1 hour at $4^{\circ} \mathrm{C}$. Beads were washed three times in wash buffer and subsequently resuspended in IX LSB. Samples were then separated by SDS-PAGE and analyzed by Western blotting using the indicated antibodies.

Single-step growth curve. Vero cells were infected with AiV at an MOI of 10. After $1 \mathrm{hr}$, the cells were washed three times in PBS to remove excess virus. At $0,2,4,6,8$, 10, or $16 \mathrm{hrs}$ pi., samples were freeze-thawed three times to release intracellular virus. The total amount of infectious virus in each sample was quantified by end-point dilution assays.

Phylogenetic analysis of KoVL proteins. KoVL protein sequences (AiVL, NP_047200.1; M-KoVL, YP_004782207.1; C-KoVL, AHK60486.1; R-KoVL, AIK67137.1; P-KoVL, YP_002473934.1; F-KoVL, AGU62946.1; B-KoVL, NP_859018.1) were aligned in MEGAX software (48) using the MUltiple Sequence Comparison by Log-Expectation (MUSCLE) method. A phylogenetic tree was created by the Maximum Likelyhood Method, using a JTT model (49), and a site coverage cut-off of $99 \%$. The final dataset included 155 positions. 


\section{References}

1. Pakos-Zebrucka K, Koryga I, Mnich K, Ljujic M, Samali A, Gorman AM. 2016. The integrated stress response. EMBO Rep 17:1374-1395.

2. Levin D, London IM. 1978. Regulation of protein synthesis: activation by double-stranded RNA of a protein kinase that phosphorylates eukaryotic initiation factor 2. Proc Natl Acad Sci U S A 75:1121-1125.

3. Harding HP, Zhang Y, Ron D. 1999. Protein translation and folding are coupled by an endoplasmic- reticulumresident kinase. Nature 397:271-274.

4. Harding HP, Zhang Y, Bertolotti A, Zeng H, Ron D. 2000. Perk is essential for translational regulation and cell survival during the unfolded protein response. Mol Cell 5:897-904.

5. Dong J, Qiu H, Garcia-Barrio M, Anderson J, Hinnebusch AG. 2000. Uncharged tRNA activates GCN2 by displacing the protein kinase moiety from a bipartite tRNA-binding domain. Mol Cell 6:269-79.

6. Chen JJ, London IM. 1995. Regulation of protein synthesis by heme-regulated eIF- $2 \alpha$ kinase. Trends Biochem Sci 20:105-8.

7. Matts RL, Schatz JR, Hurst R, Kagen R. 1991. Toxic heavy metal ions activate the heme-regulated eukaryotic initiation factor-2 $\alpha$ kinase by inhibiting the capacity of hemin-supplemented reticulocyte lysates to reduce disulfide bonds. J Biol Chem 266:12695-702.

8. Rabouw HH, Visser LJ, Passchier TC, Langereis MA, Liu F, Giansant P, van Vliet AL, Dekker JG, van der Grein SG, Saucedo JG, Anand AA, Trellet ME, Bonvin AMJJ, Walter P, Heck AJR, Groot RJ de, van Kuppeveld FJM. 2020. Inhibition of the integrated-stress-response by viral proteins that block p-eIF2•IF2B association. Nat Microbiol Online ahead of print.

9. Agol VI, Gmyl AP. 2010. Viral security proteins: counteracting host defences. Nat Rev Microbiol 8:867-78.

10. Devaney MA, Vakharia VN, Lloyd RE, Ehrenfeld E, Grubman MJ. 1988. Leader protein of foot-and-mouth disease virus is required for cleavage of the p220 component of the cap-binding protein complex. IVirol 62:4407-9.
.

Hato S V., Sorgeloos F, Ricour C, Zoll J, Melchers WJG, Michiels T, van Kuppeveld FJM. 2010. Differential IFN- $\alpha / \beta$ production suppressing capacities of the leader proteins of mengovirus and foot-and-mouth disease virus. Cell Microbiol 12:310-317

12. Borghese F, Michiels T. 2011. The Leader Protein of Cardioviruses Inhibits Stress Granule Assembly. J Virol 85:9614-9622.

13. Yamashita T, Kobayashi S, Sakae K, Nakata S, Chiba S, Ishihara Y, Isomura S. 1991. Isolation of cytopathic small round viruses with BS-C-1 cells from patients with gastroenteritis. J Infect Dis 164:954-7.

14. Visser LJ, Medina GN, Rabouw HH, de Groot RJ, Langereis MA, de Los Santos T, van Kuppeveld FJM. 2019. Foot-and-Mouth Disease Virus Leader Protease Cleaves G3BP1 and G3BP2 and Inhibits Stress Granule Formation. J Virol 93:e00922-18.

15. van Pesch V, van Eyll O, Michiels T, Lipton HL, Rundell MK. 2001. The leader protein of Theiler's virus inhibits immediate-early alpha/beta interferon production. J Virol 75:7811-7.

16. Nejepinska J, Malik R, Moravec M, Svoboda P. 2012. Deep Sequencing Reveals Complex Spurious Transcription from Transiently Transfected Plasmids. PLoS One 7:e43283.

17. Rabouw HH, Langereis MA, Knaap RCM, Dalebout TJ, Canton J, Sola I, Enjuanes L, Bredenbeek PJ, Kikkert M, de Groot RJ, van Kuppeveld FJM. 2016. Middle East Respiratory Coronavirus Accessory Protein 4a Inhibits PKR-Mediated Antiviral Stress Responses. PLoS Pathog 12:e1005982.

18. Mohammad-Qureshi SS, Haddad R, Palmer KS, Richardson JP, Gomez E, Pavitt GD. 2007. Purification of FLAG-Tagged Eukaryotic Initiation Factor 2B Complexes, Subcomplexes, and Fragments from Saccharomyces cerevisiae. Methods Enzymol 431:1-13.

19. Sekine Y, Zyryanova A, Crespillo-Casado A, Fischer PM, Harding HP, Ron D. 2015. Mutations in a translation initiation factor identify the target of a memory-enhancing compound. Science (80-) 348:1027-30.
20. Cruz JLG, Sola I, Becares M, Alberca B, Plana J, Enjuanes L, Zuñiga S. 2011. Coronavirus Gene 7 Counteracts Host Defenses and Modulates Virus Virulence. PLoS Pathog 7:e1002090.

21. Kazemi S, Yoshimura A, Su Q, Dever TE, Koromilas AE, Papadopoulou S, Li S, Matlashewski G, Wang S. 2004. Control of Subunit of Eukaryotic Translation Initiation Factor 2 (eIF2) Phosphorylation by the Human Papillomavirus Type 18 E6 Oncoprotein: Implications for eIF2 -Dependent Gene Expression and Cell Death. Mol Cell Biol 24:3415-29.

22. Bergmann M, Garcia-Sastre A, Carnero E, Pehamberger H, Wolff K, Palese P, Muster T. 2000. Influenza Virus NS1 Protein Counteracts PKR-Mediated Inhibition of Replication. J Virol 74:6203-6206.

23. Feng Z, Cerveny M, Yan Z, He B. 2007. The VP 35 protein of Ebola virus inhibits the antiviral effect mediated by double-stranded RNA-dependent protein kinase PKR. J Virol 81:182-92.

24. Chang HW, Watson JC, Jacobs BL. 1992. The E3L gene of vaccinia virus encodes an inhibitor of the interferoninduced, double-stranded RNA-dependent protein kinase. Proc Natl Acad Sci 89:4825-4829.

25. Lu Y, Wambach M, Katze MG, Krug RM. 1995. Binding of the Influenza Virus NSi Protein to Double-Stranded RNA Inhibits the Activation of the Protein Kinase That Phosphorylates the eIF-2 Translation Initiation Factor. Virology 214:222-228.

26. Kim Y, Lee JH, Park JE, Cho J, Yi H, Kim VN. 2014. PKR is activated by cellular dsRNAs during mitosis and acts as a mitotic regulator. Genes Dev 28:1310-1322.

27. Zismanov V, Chichkov V, Colangelo V, Jamet S, Wang S, Syme A, Koromilas AE, Crist C. 2016. Phosphorylatio of eIF2 $\alpha$ is a Translational Control Mechanism Regulating Muscle Stem Cell Quiescence and Self-Renewal. Cell Stem Cell 18:79-90.

28. Wang C, Tan Z, Niu B, Tsang KY, Tai A, Chan WCW, Lo RLK, Leung KKH, Dung NWF, Itoh N, Zhang MQ Chan D, Song K, Cheah E. 2018. Inhibiting the integrated stress response pathway prevents aberrant chondrocyte differentiation thereby alleviating chondrodysplasia. Elife 7:1-35.

29. Cao SS, Wang M, Harrington JC, Chuang BM, Eckmann L, Kaufman RJ. 2014. Phosphorylation of eif20 is dispensable for differentiation but required at a posttranscriptional level for paneth cell function and intestinal homeostasis in mice. Inflamm Bowel Dis 20:712-722.

30. Chen H, Song R, Wang G, Ding Z, Yang C, Zhang J, Zeng Z, Rubio V, Wang L, Zu N, Weiskoff AM, Minze LJ, Jeyabal PVS, Mansour OC, Bai L, Merrick WC, Zheng S, Shi ZZ. 2015. OLA1 regulates protein synthesis and integrated stress response by inhibiting eIF2 ternary complex formation. Sci Rep 5:13241.

31. Lin W, Bailey SL, Ho H, Harding HP, Ron D, Miller SD, Popko B. 2007. The integrated stress response prevents demyelination by protecting oligodendrocytes against immune-mediated damage. J Clin Invest 117:448-56.

32. Wang L, Popko B, Roos RP. 2014. An enhanced integrated stress response ameliorates mutant SOD1-induced ALS. Hum Mol Genet 23:2629-38.

33. Halliday M, Radford H, Sekine Y, Moreno J, Verity N, le Quesne J, Ortori CA, Barrett DA, Fromont C, Fische PM, Harding HP, Ron D, Mallucci GR. 2015. Partial restoration of protein synthesis rates by the sma molecule ISRIB prevents neurodegeneration without pancreatic toxicity. Cell Death Dis 6:e1672.

34. Moreno JA, Radford H, Peretti D, Steinert JR, Verity N, Martin MG, Halliday M, Morgan J, Dinsdale D, Ortor CA, Barrett DA, Tsaytler P, Bertolotti A, Willis AE, Bushell M, Mallucci GR. 2012. Sustained translational repression by eIF2 $\alpha-P$ mediates prion neurodegeneration. Nature 485:507-511.

35. Lourenco M V., Ferreira ST, De Felice FG. 2015. Neuronal stress signaling and eIF2 $\alpha$ phosphorylation as molecular links between Alzheimer's disease and diabetes. Prog Neurobiol 129:37-57.

36. Wong YL, Lebon L, Edalji R, Lim H Ben, Sun C, Sidrauski C. 2018. The small molecule ISRIB rescues the stability and activity of vanishing white matter disease eIF2B mutant complexes. Elife 7:e32733.

37. Hoozemans JJM, van Haastert ES, Eikelenboom P, de Vos RAI, Rozemuller JM, Scheper W. 2007. Activation of the unfolded protein response in Parkinson's disease. Biochem Biophys Res Commun 354:707-711.

38. Chou A, Krukowski K, Jopson T, Zhu PJ, Costa-Mattioli M, Walter P, Rosi S. 2017. Inhibition of the integrated stress response reverses cognitive deficits after traumatic brain injury. Proc Natl Acad Sci 114:E6420-6.

39. Nguyen HG, Conn CS, Kye Y, Xue L, Forester CM, Cowan JE, Hsieh AC, Cunningham JT, Truillet C, Tameire F, Evans MJ, Evans CP, Yang JC, Hann B, Koumenis C, Walter P, Carroll PR, Ruggero D. 2018. Development of a stress response therapy targeting aggressive prostate cancer. Sci Transl Med 10:eaar2036. 
Chapter 6 - AiVL inhibits the integrated stress response by preventing p-eIF2-mediated inhibition of eIF2B function

40. Sidrauski C, Acosta-Alvear D, Khoutorsky A, Vedantham P, Hearn BR, Li H, Gamache K, Gallagher CM, Ang KKH, Wilson C, Okreglak V, Ashkenazi A, Hann B, Nader K, Arkin MR, Renslo AR, Sonenberg N, Walter P. 2013. Pharmacological brake-release of mRNA translation enhances cognitive memory. Elife 2:e00498.

41. Takeda N, Kusuhara Y, Kobayashi N, Sasaki J, Maeno Y, Taniguchi K, Sakae K, Yamashita T. 2001. Construction of an Infectious cDNA Clone of Aichi Virus (a New Member of the Family Picornaviridae) and Mutational Analysis of a Stem-Loop Structure at the 5' End of the Genome. J Virol 75:8021-30.

42. De Jong AS, Wessels E, Dijkman HBPM, Galama JMD, Melchers WJG, Willems PHGM, Van Kuppeveld FJM. 2003. Determinants for membrane association and permeabilization of the coxsackievirus $2 \mathrm{~B}$ protein and the identification of the Golgi complex as the target organelle. J Biol Chem 278:1012-21.

43. Shevchenko A, Wilm M, Vorm O, Mann M. 1996. Mass spectrometric sequencing of proteins from silverstained polyacrylamide gels. Anal Chem 68:850-8.

44. Cristobal A, Hennrich ML, Giansanti P, Goerdayal SS, Heck AJR, Mohammed S. 2012. In-house construction of a UHPLC system enabling the identification of over 4000 protein groups in a single analysis. Analyst 137:3541-8.

45. Käll L, Canterbury JD, Weston J, Noble WS, MacCoss MJ. 2007. Semi-supervised learning for peptide identification from shotgun proteomics datasets. Nat Methods 4:923-5.

46. Choi H, Liu G, Mellacheruvu D, Tyers M, Gingras AC, Nesvizhskii AI. 2012. Analyzing protein-protein interactions from affinity purification-mass spectrometry data with SAINT, p. chapter 8, unit 15. In Current Protocols in Bioinformatics.

47. de Vries RP, de Vries E, Bosch BJ, de Groot RJ, Rottier PJM, de Haan CAM. 2010. The influenza A virus hemagglutinin glycosylation state affects receptor-binding specificity. Virology 403:17-25.

48. Kumar S, Stecher G, Li M, Knyaz C, Tamura K. 2018. MEGA X: Molecular evolutionary genetics analysis across computing platforms. Mol Biol Evol 35:1547-9.

49. Jones DT, Taylor WR, Thornton JM. 1992. The rapid generation of mutation data matrices from protein sequences. Bioinformatics 8:275-82. 
Supplementary Figures

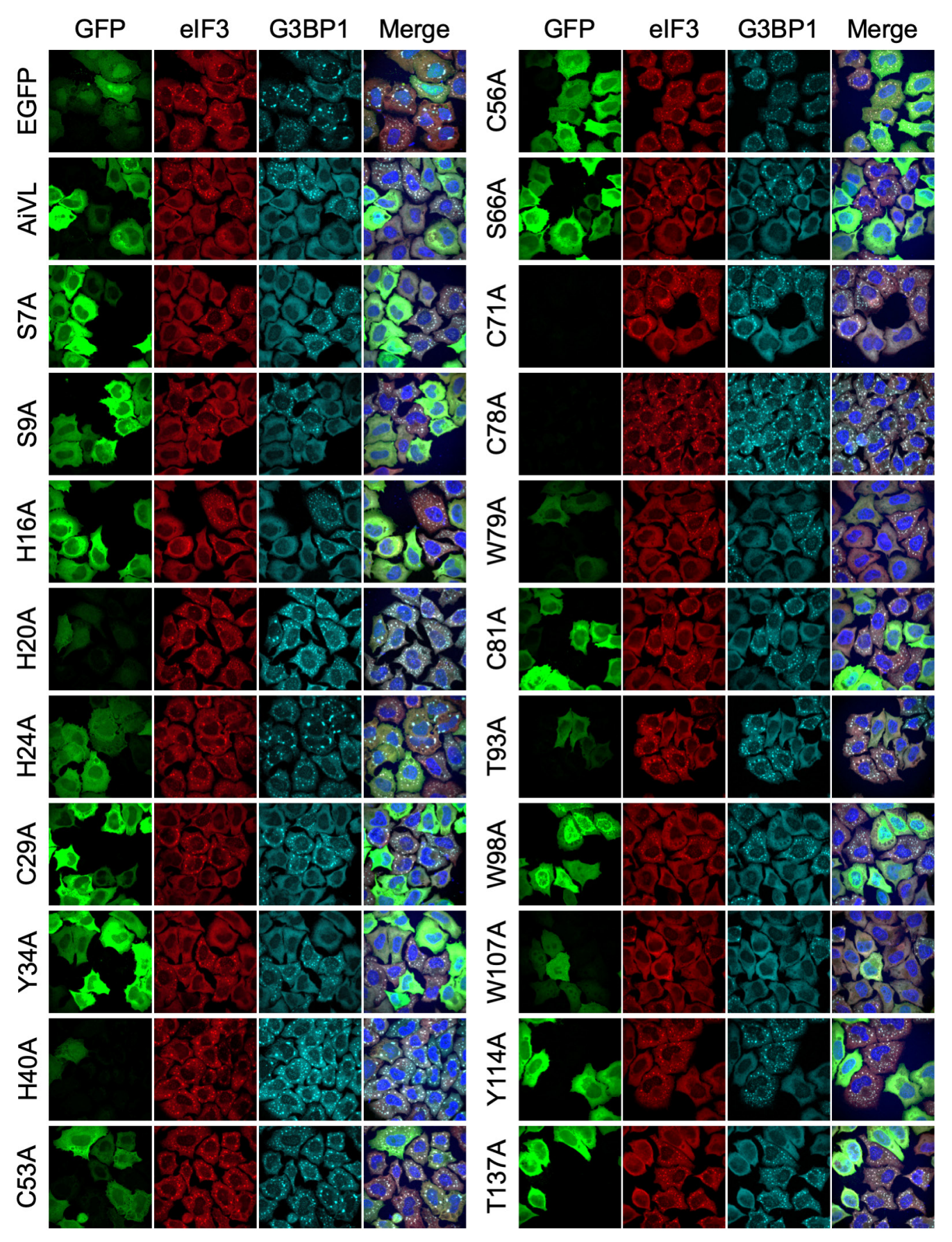

< Figure S1: IFA analysis of SG inhibition by the full panel of AiVL point mutants. The indicated ECFP-tagged AiVL mutant proteins were transiently overexpressed in Hela cells. Next day cells were treated with $250 \mathrm{uM}$ arsenite for $1 \mathrm{hr}$ and SC formation was analyzed by IFA using antibodies directed against SC markers $\mathrm{C}_{3} \mathrm{BP}_{1}$ and elF3.

A

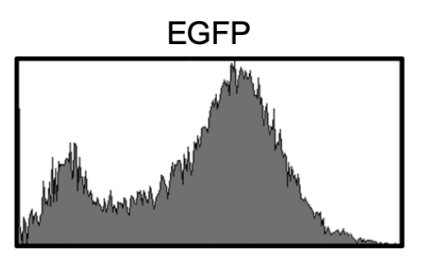

AcP10-EGFP

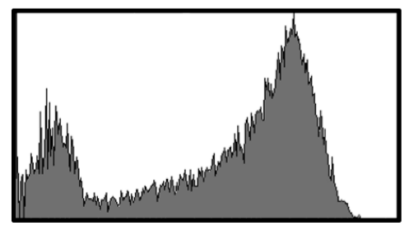

AiVL-EGFP

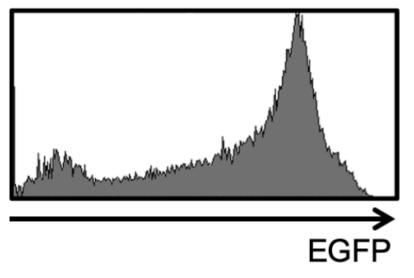

B

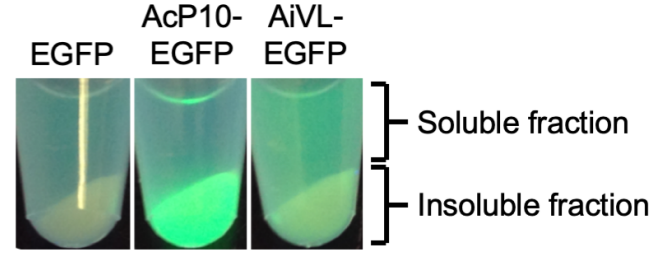

Figure S2: Transient AiVL overexpression yields high concentrations of soluble protein. (A) Flowcytometry analysis of HeLa cells transiently overexpressing the indicated proteins. (B) In parallel, cells were lyzed in lysis buffer, and cell debris was spun down. In the resulting samples, containing an insoluble pellet and a soluble supernatant fraction, ECFP content was visualized under UV-light. 


\section{Chapter 7}

General Discussion

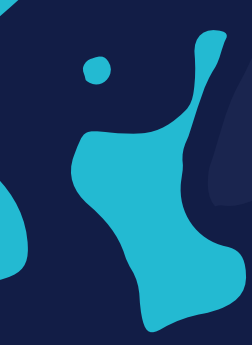

○ 
The Picornaviridae are a large virus family consisting of 47 genera and 110 species and amongst these viruses are several human and veterinary pathogens. Viral infection is recognized by the host and triggers an antiviral response, which is centered around the production of type I interferons and the antiviral effects of interferon stimulated genes (ISGs). Meanwhile, viruses try to evade the antiviral effects of this response. Picornaviruses are known to predominantly depend on their security proteins ( $2 \mathrm{~A}$ and L) to evade antiviral responses, although many of the underlying molecular mechanisms remain to be elucidated. In this thesis, we studied how several picornaviruses evade the induction of type I interferons and the antiviral activities of the ISG PKR. PKR acts as a dsRNA sensor and activator of the cellular stress response. In chapter 2-4 we studied the mechanisms employed by foot-and-mouth disease virus to evade the induction of type I interferons and the cellular stress response. In chapter 5 we investigated how enteroviruses suppress both antiviral pathways and in chapter 6 we explored how kobuviruses evade the cellular stress response. Here, the main findings are summarized and common themes in the modes of action of the different picornavirus security proteins are discussed.

\section{Summary}

In chapter 2, we investigated whether and how members of the Aphthovirus genus suppress the cellular stress response. We found that FMDV efficiently suppresses stress granule (SG) formation, which we found to be dependent on the catalytic activity of the Leader protease $\left(\mathrm{L}^{\text {pro }}\right)$. We generated chimeric EMCVs that encoded either FMDV L pro or ERAV L pro $^{\text {pro }}$ (FDV's closest relative), to demonstrate that $\mathrm{L}^{\text {pro }}$ does not suppress PKR signaling but affects SG formation. We also demonstrated that $\mathrm{L}^{\text {pro }}$ cleaves the SG scaffolding protein $\mathrm{G}_{3} \mathrm{BP}_{1}$ and $\mathrm{G} 3 \mathrm{BP} 2$, and we showed that G3BP1 cleavage occurs upon FMDV infection.

In chapter 3, we identified FMDV $L^{\text {pro }}$ as a deISGylase and showed that $L^{\text {pro }}$ has very little deubiquitinase activity. We demonstrate this preference of $\mathrm{L}^{\text {pro }}$ for ISG15 in vitro, but also in infected cells. Furthermore, we demonstrate that $\mathrm{L}^{\mathrm{pro}}$ is a unique deISGylase, leaving a GlyGly modification on the lysine of target proteins, whereas canonical deISGylases hydrolyze the isopeptide linkage between ISG15 and the lysine of the target protein. The presence of uniquely modified lysines in proteins from FMDV-infected cells can potentially be used to develop new FMDV detection strategies, which can efficiently distinguish between infected and vaccinated animals.

The findings in chapter 3 also initiated the research described in chapter 4. FMDV suppresses the production of type I interferons by suppressing RLR signaling but also by directly affecting translation of IFN- $\alpha / \beta$. FMDV L $L^{\text {pro }}$ inhibits cap-dependent translation by cleaving eIF4G and this likely affects IFN- $\alpha / \beta$ translation. FMDV also requires $L^{\text {pro }}$ to suppress RLR signaling but the underlying mechanism remains to be fully elucidated as several mechanisms have been proposed over the years. $\mathrm{L}^{\text {pro }}$ has been suggested to degrade the transcription factors IRF3, IRF7 and $\operatorname{NFKB}(1,2)$. Additionally, $\mathrm{L}^{\text {pro's }}$ DUB activity has been suggested to interfere with RLR signaling (3). While $\mathrm{L}^{\text {pro }}$ does have DUB activity upon overexpression, we found no evidence of significant DUB activity during viral infection (chapter 3 ). In chapter 4 , we investigated whether the deISGylase activity of $L^{\text {pro }}$ contributes to the suppression of RLR signaling or whether alternative mechanisms contribute to this ability of $L^{\text {pro }}$. First, we demonstrated that $L^{\text {pro }}$ cleaves the RLR signaling proteins TBK 1 and MAVS. Introduction of specific mutations in $\mathrm{L}^{\text {pro }}$ subsequently allowed us to identify amino acids that are essential for either L $^{\text {pros }}$ deISGylase/DUB activity or its proteolytic activity towards RLR signaling proteins. We demonstrated that a $L^{\text {pro }}$ mutant that is severely impaired in its deISGylase activity can still suppress IFN- $\beta$ gene transcription, while a $\mathrm{L}^{\text {pro }}$ mutant that is severely impaired in the cleavage and degradation of RLR signaling proteins cannot. Our combined observations suggest that $\mathrm{L}^{\text {pro }}$ suppresses RLR signaling independently from its deISGylase activity and point to a role for the cleavage of RLR signaling proteins.

In chapter 5, we focused on members of the Enterovirus genus and investigated their ability to evade the induction of IFN- $\alpha / \beta$ gene expression and the cellular stress response. The two enterovirus proteases (i.e. $2 \mathrm{~A}^{\text {pro }}$ and $3 \mathrm{C}^{\text {pro }}$ ) have been suggested to help the viruses evade antiviral responses. We compared the ability of individual enterovirus proteases of representative members of the enterovirus species EV-A to EV-D to interfere with the induction of IFN- $\alpha / \beta$ gene expression as well as cellular stress response activation. This comprehensive analysis revealed that enterovirus $2 \mathrm{~A}^{\text {pro }}$ of multiple enteroviruses is able to suppress IFN- $\alpha / \beta$ gene expression and inhibit SG formation. $2 \mathrm{~A}^{\text {pro }}$ inhibits $S G$ formation but does not affect cellular stress response signaling (e.g. counteract the phosphorylation of PKR or eIF2 $\alpha$ ), suggesting that $2 \mathrm{~A}^{\text {pro }}$ targets SG assembly. SGs themselves have been suggested to act as a signaling platform that is critical for the induction of IFN- $\alpha / \beta$ expression $(4,5)$. Therefore, we investigated whether the effects of $2 \mathrm{~A}^{\text {pro }}$ on RLR signaling depend on $2 \mathrm{~A}^{\text {pro's }}$ ability to counteract SG assembly. Using cells that are deficient in SG formation (i.e. PKR k.o. MEFs and $\mathrm{G}_{3} \mathrm{BP}_{1} / \mathrm{G}_{3} \mathrm{BP}_{2}$ double k.o. HeLa cells), we demonstrated that the inability of cells to form SGs reduces IFN $-\alpha / \beta$ gene expression $\sim 10$-fold. Thus, we provide evidence that SG formation contributes to, but is not critical for, RLR signaling. In addition, we demonstrated that $2 \mathrm{~A}^{\text {pro }}$ suppresses IFN- $\alpha / \beta$ gene expression $\sim 500$-fold, suggesting that $2 \mathrm{~A}^{\text {pro }}$ has additional mechanisms to directly target RLR signaling.

In chapter 6 we investigated how members of the Kobuvirus genus evade the cellular stress response. Thus far, viruses counteract the stress response by directly antagonizing SG 


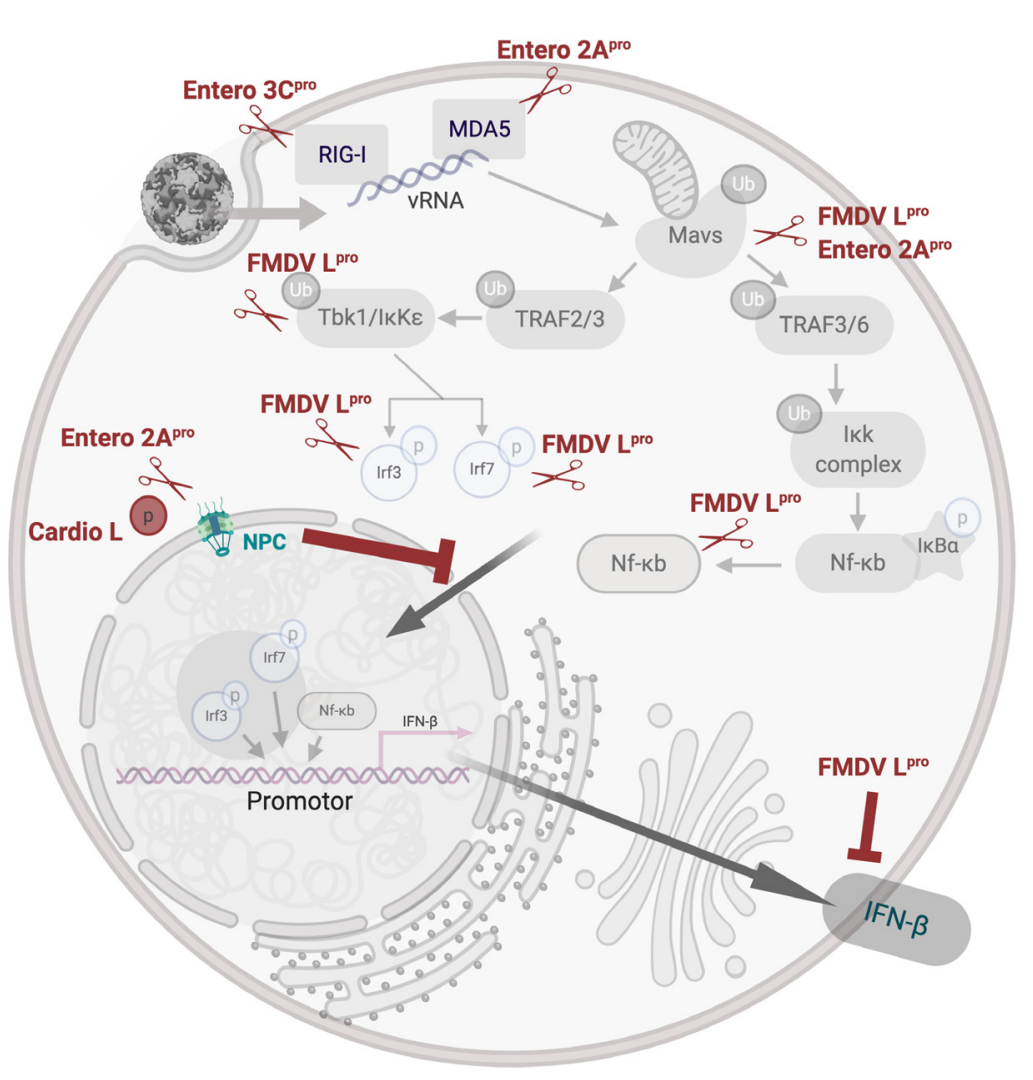

Figure 1: The RLR signaling pathway is targeted by multiple picornavirus proteins. This schematic representation of the RLR signaling pathway has been introduced in chapter 1 . This version indicates which signaling components are targeted by enterovirus $2 A^{\text {pro }}$, enterovirus $3 C^{p r o}, F M D V L^{\text {pro }}$ and the cardiovirus $L$ protein. This figure was made with BioRender.

formation, or by preventing and/or reversing eIF2 phosphorylation. Aichivirus, through the activity of L, allows ongoing translation in the presence of high levels of p-eIF2. Aichivirus $\mathrm{L}$ binds eIF2 $\mathrm{B}$ and this prevents association of p-eIF2 to eIF2B. Hereby, p-eIF2 no longer interferes with the interaction between non-phosporylated eIF2 and eIF2B, and translation can continue. Mouse kobuvirus L, which is the closest relative of aichivirus L, was the only other kobuvirus $\mathrm{L}$ that rescued translation upon high levels of p-eIF2. The inability of other kobuviruses leaders to interfere with the cellular stress response suggests that this ability was a recent gain-of-function event and that kobuvirus leader likely has another primary function.

Figures 1 and 2 provide a schematic representation of the RLR signaling cascade (fig. I) and the cellular stress response (fig. 2), and indicate how the security proteins studied in this thesis counteract these antiviral responses. In the experiments described in this thesis, we frequently

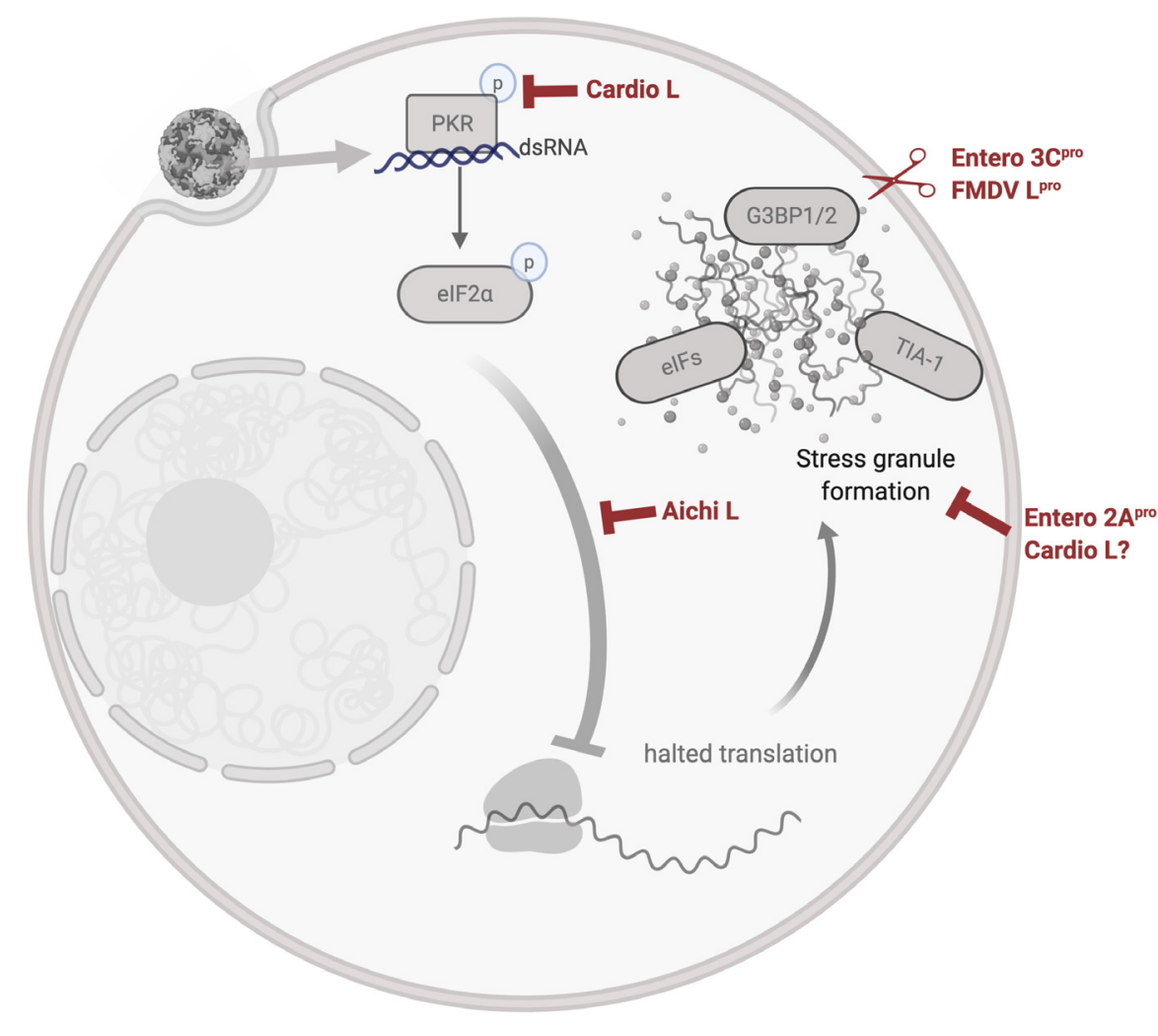

7

Figure 2: The cellular stress response is targeted by multiple picornavirus proteins. This schematic representation of the cellular stress response has been introduced in chapter 1 . This version indicates where in the pathway enterovirus $2 A^{\text {pro }}$ enterovirus $3 C^{\text {pro }}$ FMDV L pro cardiovirus $L$ and Aichivirus $L$ counteract the response. This figure was made with BioRender.

made use of the cardiovirus EMCV and an EMCV mutant virus (EMCV-L $\mathrm{L}^{\mathrm{Zn}}$ ) that carries inactivating mutations in its antagonist of the innate antiviral responses $\left(\mathrm{EMCV}-\mathrm{L}^{\mathrm{Zn}}\right)$ as positive and negative controls. Therefore, the mechanisms of cardiovirus $L$ have been included in figures 1 and 2. Cardiovirus $L$ is a well described antagonist of RLR signaling as well as the cellular stress response. Cardiovirus L efficiently suppresses RLR signaling by preventing or counteracting IRF3 phosphorylation, dimerization and translocation to the nucleus (collectively referred to as IRF3 activation) (6-8). In addition, cardiovirus L inhibits the cellular stress response at, at least, two stages. Cardiovirus L inhibits the phosphorylation of PKR $(9,10)$ and thereby prevents the downstream phosphorylation of eIF2 and the formation of SGs. L also prevents the formation of SGs upon addition of sodium arsenite (10, 11), although under these conditions L fails to inhibit eIF2 phosphorylation (10). Thus, L inhibits PKR phosphorylation and L counteracts the stress response at a step between eIF2 phosphorylation and SG formation. 


\section{Common themes in the mechanisms used by picornavirus security proteins}

The picornavirus security proteins are the least conserved amongst the picornavirus proteins and the mechanisms they employ are incredibly diverse. We identified several intriguing parallels in the mechanisms used by the different security proteins, which could indicate specific vulnerable sites in the antiviral responses that can be exploited by viruses. In this section these common themes and their implications are discussed.

\section{Counteracting SG assembly; a matter of targeting $\mathrm{G}_{3} \mathrm{BP}_{1}$ and eIF $4 \mathrm{G}$ ?}

In chapter 2 , we describe that FMDV suppresses $S G$ formation via its leader protease ( $L^{\text {pro }}$ ), and show that suppression of SG formation depends on $\mathrm{L}^{\text {pro's }} \mathrm{s}$ catalytic activity. We also show that $\mathrm{L}^{\text {pro }}$ cleaves the SG scaffolding proteins $\mathrm{G}_{3} \mathrm{BP} 1$ and $\mathrm{G}_{3} \mathrm{BP} 2$. We do not demonstrate a causal relationship between $S G$ suppression and $\mathrm{G}_{3} \mathrm{BP}_{1}$ and $\mathrm{G}_{3} \mathrm{BP} 2$ cleavage, but a causal relationship between $\mathrm{G}_{3} \mathrm{BP}_{1}$ cleavage and $\mathrm{SG}$ suppression has been suggested for a number of enteroviruses (12-14). However, in chapter 5 we showed that the cleavage of G3BP1 by enterovirus $3 \mathrm{C}^{\text {pro }}$ only modestly reduced SG formation. Instead, we and others (chapter 5 and (15)) demonstrated that enterovirus $2 \mathrm{~A}^{\text {pro }}$ very efficiently suppressed SG formation. Thus far, enterovirus $2 \mathrm{~A}^{\text {pro }}$ has not been demonstrated to cleave any known SG scaffolding proteins and thus it is debatable whether there is a causal relationship between G3BPI cleavage and inhibition of SG formation.

Importantly, in chapter 5 we also showed that both G3BP1 and G3BP2 need to be depleted to abolish SG formation. In contrast to enterovirus $3 \mathrm{C}^{\text {pro }}$, FMDV $\mathrm{L}^{\text {pro }}$ cleaves both $\mathrm{G} 3 \mathrm{BPI}$

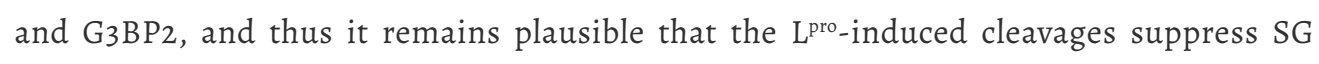
formation. Enterovirus $3 \mathrm{C}^{\text {pro }}$ only targets $\mathrm{G}_{3} \mathrm{BP}$ and while this does reduce the number of SGs, it is insufficient to completely prevent SG formation. Without evidence of a causal

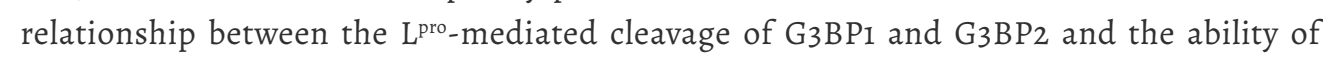
$L^{\text {pro }}$ to suppress SG formation - for example by determining whether $L^{\text {pro }}$ suppresses $S G$ formation in cells expressing cleavage resistant $\mathrm{G}_{3} \mathrm{BP} 1$ and $\mathrm{G}_{3} \mathrm{BP} 2$ - we should also consider alternative mechanisms via which $L^{\text {pro }}$ could suppress SG formation.

It was recently suggested that enterovirus $2 \mathrm{~A}^{\text {pro }}$ breaks a newly identified interaction between G3BP1 and eIF4G (16). Breaking of this interaction, which was shown to depend on $2 \mathrm{~A}^{\text {pro's }}$ catalytic activity, was suggested to be the mechanism via which $2 \mathrm{~A}^{\text {pro }}$ inhibits SG formation. One would expect the cleavage of eIF4G by $2 \mathrm{~A}^{\text {pro }}$ to cause the loss of the eIF4G-G3BPi interaction. Counterintuitively, Yang et al. showed that $2 \mathrm{~A}^{\text {pro }}$ also disrupts the interaction between $\mathrm{G}_{3} \mathrm{BP}_{1}$ and a 'cleavage resistant' eIF4G (eIF4G G689E) and $2 \mathrm{~A}^{\text {pro }}$ continued to suppress SG formation under these conditions (16). EMCV L, which lacks catalytic activity, was also shown to disrupt the eIF4G-G3BPI interaction (16). This could explain the observation that cardiovirus L can counteract sodium arsenite-induced SG formation, but not sodium arsenite-induced eIF2 phosphorylation (10, 11). Collectively, these observations support the idea that both $2 \mathrm{~A}^{\text {pro }}$ and cardio L target $\mathrm{SG}$ assembly. This hypothesis may be investigated further with the use of pateamine $\mathrm{A}$, an inhibitor of eIF4A and thus a molecule that blocks translation initiation independently of p-eIF2 (17, 18). At the present time, the molecular mechanism(s) underlying the suppression of SG formation by enterovirus $2 \mathrm{~A}^{\text {pro }}$, cardiovirus $\mathrm{L}$ and FMDV $\mathrm{L}^{\text {pro }}$ remain only partially understood. The available data hint to a possible role for targeting the interaction between eIF4G and G3BPI by multiple picornavirus security proteins, but this requires further investigation.

To fully comprehend the strategies employed by viruses to counteract SG formation, one needs to understand the driving forces of SG formation. In recent years, several unbiased large-scale analyses have been performed to investigate the protein and RNA composition of "core" SGs $(19,20)$. In these studies, G3BP1 was precipitated and the precipitated complexes were subsequently analyzed using mass spectrometry or RNA-seq. A recent study combined data of a genome-wide RNAi screen with a comprehensive bioinformatics analysis of the previous SG datasets to generate a "core SG network" consisting of 36 protein (21). This network includes most proteins previously claimed to be essential for SG formation (e.g. TIA- 1 and G3BPs), but notably lacks eIF4G. Out of the 36 proteins within the core SG network, only the double knock-out of G3BP1 and G3BP2 resulted in a cell line completely devoid of SG formation upon treatment with sodium arsenite, which is consistent with the results described in chapter 5 of this thesis. The high "betweenness centrality" of the G3BPs in the core SG network suggested that they are an essential driving force for the formation of SGs, at least during sodium arsenite-induced cellular stress. Subsequent analysis showed that G3BP1 and G3BP2 can undergo RNA-dependent phase separation. The ability of G3BP1 and $\mathrm{G} 3 \mathrm{BP} 2$ to undergo phase separation was dependent on their RNA binding domain (RBD), which itself consists of an RNA recognition motif (RRM) and the intrinsically disordered region 3 (IDR3). Simultaneously, their NTF2-like domain was found to be important for their ability to dimerize. The ability of G3BPs to dimerize as well as their ability to undergo phase separation were identified as essential drivers underlying SG formation. Meanwhile, the additional proteins of the core SG network were found to lower the thresholds of G3BP phase separation and thereby positively influence SG formation. These new insights into the biophysical processes underlying SG formation highlight the central roles of G3BP1 and $\mathrm{G}_{3} \mathrm{BP} 2$ and support the previously discussed mechanisms via which enterovirus $2 \mathrm{~A}^{\mathrm{pro}}$, cardiovirus L and FMDV L ${ }^{\text {pro }}$ could counteract SG formation. 
Similarly, one must understand the "requirements" for an RNA molecule to be incorporated into SGs. RNAseq analysis of the SG "core" revealed that SGs primarily consist of mRNA; $78 \%$ of the RNA from U-2 OS cell derived SGs is mRNA, this further increases to $95 \%$ when yeast derived SGs are analyzed (20). Meanwhile, a U-2 OS cell transcriptome consists of only $17 \%$ mRNA, while the other $83 \%$ is made up of various ncRNA molecules. Furthermore, the mRNAs that are enriched in the SG core are not the overly abundant mRNAs (e.g. actin), have less ribosome density and are rather long (average length is $7.1 \mathrm{~kb}$ ) (20). One should also investigate a possible role for eIFs bound to mRNA in the recruitment of mRNAs to SGs. Several eIFs have been found to localize to SGs. The eIFs found in SGs are reminiscent of the $48 \mathrm{~S}$ pre-initiation complexes, with the notable exception of eIF2. These eIFs are suggested to be coordinately recruited to SGs, as they remain bound to mRNAs that are recruited to $S G s$ (22). Intriguingly, eIF4E carrying an inactivating mutation in its eIF4G/ eIF4E-BP interaction domain failed to localize to SGs, while wt eIF4E does localize to SGs $(23,24)$. mRNAs bound by the mutant eIF4E lack all other eIFs and fail to recruit the preinitiation complex. Failure of these eIF4E-mRNA complexes to localize to SGs could suggest that mRNAs lacking certain eIFs are not incorporated in SGs.

Cleavage of eIF4G by $2 \mathrm{~A}^{\text {pro }}$ separates the eIF4E-interaction domain (i.e. eIF4Gs N-terminus) from the domain that interacts with further eIFs and thus facilitates pre-initiation complex recruitment (eIF4Gs C-terminus). An mRNP carrying just eIF4E and the eIF4G N-terminal cleavage fragment lacks most eIFs and is nearly identical to a mRNP containing the eIF4E interaction domain mutant discussed above. Which eIFs need to be bound to a mRNA for said mRNAs to be recruited to SGs unfortunately remains incompletely understood. Therefore, it remains unclear whether mRNPs that contain the eIF4G N-terminal cleavage product rather than full length eIF4G, and thus lack other eIFs such as eIF3 and eIF4A, are recruited to SGs. Importantly, enterovirus $2 \mathrm{~A}^{\text {pro }}$ and FMDV L $L^{\text {pro }}$ cleave eIF4GI and eIF4GII at nearly the same place, the respective cleavage sites are located just a few residues apart (25). Thus, one would suggest that eIF4G cleavage by either protease has a similar effect on SG formation. It is worthwhile to investigate whether FMDV L pro's ability to cleave eIF4G contributes to its ability to suppress SG formation. This may ultimately shed light on the possible causality between eIF4G cleavage and counteracting SG assembly.

Of note, we and others observed that expression of enterovirus $2 \mathrm{~A}^{\text {pro }}$ can induce formation of 'atypical' SGs that contain some, but not all, bona fide SG marker proteins (chapter 5 and $(15,26))$. The atypical SGs were suggested to be induced by the sudden surplus of nontranslating mRNPs, as a result of eIF4G cleavage (26). As infection continues, the atypical SGs dissolve and the cells subsequently fail to form SGs. Expression of FMDV or ERAV L pro resulted in the accumulation of similar small SGs that subsequently disappeared (chapter 2). A scenario where cleavage of eIF4G first induces formation of atypical SGs, and where
eIF4G subsequently plays a role in inhibiting the formation of bona fide SGs, illustrates the complexity of virus-host interactions. A recent $2 \mathrm{~A}^{\text {pro }}$ interactome analysis identified the eIF3 complex as an important interaction partner of $2 \mathrm{~A}^{\text {pro }}(27)$. Whether and how the interaction between $2 \mathrm{~A}^{\text {pro }}$ and eIF3 contributes to the ability of $2 \mathrm{~A}^{\text {pro }}$ to evade $\mathrm{SG}$ formation, or promotes the formation of atypical SGs, remains to be determined. The interaction between $2 \mathrm{~A}^{\text {pro }}$ and eIF3 should be considered when investigating how $2 \mathrm{~A}^{\text {pro }}$ suppresses $\mathrm{SG}$ formation.

\section{Promoting viral translation in the presence of phosphorylated eIF2}

Canonical translation is inhibited by the phosphorylation of eIF2. This could also affect viral translation and thus phosphorylation of eIF2 is potentially antiviral. To successfully replicate, viruses overcome the negative effects of phosphorylation of eIF2. Many viruses (i.a. herpes simplex virus, respiratory syncytial virus, human immunodeficiency virus and transmissible gastroenteritis virus) rescue viral translation by preventing or reversing the phosphorylation of eIF2 (28-31). Another strategy is to ensure that viral translation is resistant to the inhibitory effects of p-eIF2.

Several viruses have evolved noncanonical translation mechanisms that are independent of (most of) the eukaryotic translation initiation factors, which ensures viral translation to continue despite the phosphorylation of eIF2. One example is the translation of the subgenomic RNA (sgRNA) molecule of alphaviruses that is generated during genome replication, which occurs independent of translation initiation factors eIF2 and eIF4A (32-35) during the later stages of infection, when eIF2 is phosphorylated. Translation of sgRNA in the presence of p-eIF2 was found to depend on a hairpin structure in the coding region, known as the DLP (35). The DLP is located downstream of the initiator AUG and was suggested to stall a scanning ribosome exactly at this codon, thereby facilitating translation initiation independently of eIF2(35). Another example is the Cricket paralysis virus intergenic region IRES (CrPV IRES), which facilitates translation independently of all translation initiation factors. The $40 \mathrm{~S}$ ribosomal subunit can directly interact with the CrPV IRES, and this signals for recruitment of the 60 S subunit to facilitate 80 S formation $(36,37)$. The generated $80 \mathrm{~S}$ ribosome directly facilitates translation 'initiation' from a nonAUG codon via translation elongation factors $1 \mathrm{~A}$ and 2 (38). Translation from the CrPV IRES completely bypasses the intricate process of eukaryotic translation initiation and is thereby resistant to inhibition by p-eIF2.

Picornavirus translation is also directed from an IRES, in fact the poliovirus and EMCV IRESes were amongst the first discovered IRES structures. Translation initiation from the picornavirus IRESs is slightly more complex than CrPV IRES-driven translation. Picornavirus translation starts from a downstream AUG instead of directly at the IRES, 
this requires the ribosome to assemble at the IRES structure and subsequently scan for the start codon. Thus far, all identified picornavirus IRESes need most translation initiation factors, including eIF2, to direct translation in vitro (39-42). Despite the need for eIF2 in in vitro IRES-mediated translation, picornaviruses efficiently translate and replicate in vivo and in cellulo under conditions of cellular stress. This suggests that they have evolved mechanisms either to prevent the phosphorylation of eIF2 or that viral translation is resistant to the inhibitory effects of p-eIF2.

During enterovirus infection PKR and eIF2 are phosphorylated (13, 15, 43, 44), suggesting that enteroviruses facilitate viral translation despite high levels of p-eIF2. Indeed, enterovirus translation was reported to be resistant to the inhibiting effects of p-eIF2 $(43,45)$. Treatment of cells with sodium arsenite, applying heat shock, or addition of an eIF2 $\alpha$ phosphatase-inhibitor increased the level of p-eIF2 without significantly affecting poliovirus translation at the mid-to-late stages of infection. Translation of CVB3 was found to be similarly resistant to p-eIF2, demonstrating that this ability is likely to be conserved amongst enteroviruses (45). Interestingly, the earliest viral translation (at 2 hours post infection) was affected by the artificial induction of eIF2 phosphorylation, suggesting that enteroviruses switch from canonical translation to a p-eIF2 resistant alternative mode of translation. In contrast to viral translation, expression of a IRES-containing luciferase reporter construct is inhibited by p-eIF2 $(43,45)$. This observation illustrates that translation from a type II IRES is only resistant to p-eIF2 in the context of viral infection, which suggests that a viral product mediates the switch from canonical to p-eIF2 resistant translation. Because the early viral translation is affected by p-eIF2, it is estimated that this switch occurs around 2 hours post infection, which coincides with the accumulation of the viral proteins in the cell (45). Enteroviral proteases $2 \mathrm{~A}^{\text {pro }}$ and $3 \mathrm{C}^{\text {pro }}$ have both been implicated to mediate the switch from p-eIF2 sensitive to p-eIF2 resistant viral translation.

Enterovirus $3 C^{\text {pro }}$ targets eIF5B (46) and this has been suggested to facilitate the switch to p-eIF2 resistant translation (45). eIF5B is a homologue of prokaryotic IF2 that facilitates $80 \mathrm{~S}$ assembly and stabilizes the initiator tRNA ${ }^{\text {Met }}$ when eIF2 has been released. Ectopic overexpression of wt eIF5 $B$ stimulated poliovirus translation in the presence of sodium arsenite. Interestingly, ectopic expression of cleavage resistant eIF5B (eIF5B Q478E) impaired viral translation at 6 hours post infection, which suggests that efficient viral translation requires the cleavage of eIF5B (45). In agreement with this observation, the eIF5B C-terminal cleavage fragment was shown to positively influence IRES translation $(\sim 2$-fold increase) in the presence of sodium arsenite (45). Eukaryotic eIF5B contains a large $\mathrm{N}$-terminal extension that is absent in its prokaryotic (IF2) and archaeal (aIF5B) homologues, and it has been shown that full length as well as $\mathrm{N}$-terminally truncated yeast eIF5B can interact with initiator tRNA ${ }^{\text {Met }}$, albeit less efficiently than eIF2 (47). White et.al. suggested that the C-terminal cleavage product of eIF5B behaves more like prokaryotic IF2 and their data clearly demonstrate that cleavage of eIF5B by $3 \mathrm{C}^{\text {pro }}$ is needed to facilitate p-eIF2 resistant translation (45).

Hepatitis C virus (HCV) and classical swine fever virus (CSFV) were reported to exploit full length eIF5B to overcome the negative effects of p-eIF2 on viral translation $(48,49)$. Translation initiation from both the HCV and CSFV IRES is rather straightforward; the $40 S$ subunit directly interacts with the IRES, in a mechanism similar to the CrPV IRES $(50,51)$. In the case of the HCV and CSFV IRES, translation initiation does depend on the ternary complex (eIF2-GTP-tRNA ${ }_{i}^{\text {Met }}$ ) and eIF3 and thus viral translation, theoretically, is sensitive to the phosphorylation of eIF2 (51-54). However, for both IRESes it has been demonstrated that they allow functional replacement of eIF2 by eIF 5 B, in particular under conditions where eIF2 is phosphorylated $(48,49)$. The possibility to hijack eIF5B to ensure viral translation despite the phosphorylation of eIF2 may illustrate a vulnerable site in the hosts antiviral defenses that many viruses, including enteroviruses, take advantage off.

Enterovirus $2 \mathrm{~A}^{\text {pro }}$ was also suggested to facilitate the switch to a p-eIF2 resistant mode of viral translation (43). In sodium-arsenite treated cells, ectopic overexpression of $2 \mathrm{~A}^{\text {pro }}$ rescued IRES-mediated translation, while ectopic overexpression of the other viral proteins, including $3 \mathrm{C}^{\text {pro }}$, did not. The ability of $2 \mathrm{~A}^{\text {pro }}$ to rescue translation upon high levels of p-eIF2 correlates with the catalytic activity of $2 \mathrm{~A}^{\text {pro }}$, as determined by the efficiency of eIF $4 \mathrm{G}$ cleavage. Expression of the eIF4G N- and C-terminal cleavage products was not sufficient to rescue viral translation, suggesting that $2 \mathrm{~A}^{\text {pro }}$ cleaves another unidentified host protein to switch from canonical translation to a p-eIF2 resistant mode of translation. Whether enterovirus $2 \mathrm{~A}^{\text {pro }}$ and $3 \mathrm{C}^{\text {pro }}$ act in a synchronized manner to facilitate viral translation when eIF2 is phosphorylated has thus far not been explored.

FMDV $L^{\text {pro }}$ was also reported to facilitate $\mathrm{p}$-eIF2 resistant IRES-driven translation. $\mathrm{L}^{\text {pro }}$ supports translation in the presence of sodium arsenite from both type I and type II IRES structures, and this ability coincides with cleavage of eIF4G by $\mathrm{L}^{\text {pro }}(55) . \mathrm{FMDV} \mathrm{L}^{\text {pro }}$ and enterovirus $2 \mathrm{~A}^{\text {pro }}$ are structurally different enzymes but share many functions; both cleave translation initiation factor eIF4G (56-58), both reduce type I interferon gene expression (chapters 4,5 and $(6,59,60)$ ), and both have been implicated in the suppression of SG formation (chapters 2, 5 and (15)). Thus far there is evidence that the catalytic activity of both viral proteases facilitates the switch in translation mechanism, but the exact proteolytic target remains to be elucidated. Furthermore, there is evidence that cardiovirus translation continues despite high levels of p-eIF2, although this has not been linked to a specific viral protein (61). 
Meanwhile, we uncovered that Aichi L can counteract the cellular stress response at its core. Aichi L interferes with the p-eIF2 • eIF2B interaction, thereby allowing the continued formation of new ternary complex despite the presence of high levels of p-eIF2. Viral antagonists of the cellular stress response identified thus far either prevented or reversed the phosphorylation of eIF2 (28-31). Aichi L can antagonize the cellular stress response and rescue translation, irrespective of the nature of the stressor and stress severity. Therefore, this is arguably one of the most efficient ways to block the cellular stress response described to date. This novel mechanism of cellular stress response antagonism has been simultaneously identified for another viral protein, the accessory protein 10 (AcP10) of beluga whale coronavirus (BW-CoV) (62). Both viral proteins appear to have a different primary function and acquired stress response antagonism via recent gain-offunction mutations. The acquisition of a novel stress response antagonism strategy by a picornavirus and coronavirus is an interesting example of convergent evolution.

These collective observations suggest that rescuing viral translation from the inhibitory effects of phosphorylated eIF2 may be a strategy commonly employed by many picornaviruses, albeit via various underlying molecular mechanisms. The mechanisms underlying enterovirus $2 \mathrm{~A}^{\text {pro }} / 3 \mathrm{C}^{\text {pro }}$ and FMDV L pro-mediated p-eIF2-resistant IRES-driven translation remain to be elucidated, but clearly differ from the mechanism used by aichivirus L. $2 \mathrm{~A}^{\text {pro }}, 3 \mathrm{C}^{\text {pro }}$, and $\mathrm{L}^{\text {pro }}$ have been suggested to rely on their catalytic activity to restore IRES translation. Aichivirus $L$ lacks catalytic activity and instead relies on a protein-protein interaction between itself and eIF2B to circumvent the inhibitory effects of the cellular stress response. Meanwhile, the viral protein involved in facilitating non-canonical translation in cardiovirus infection remains to be identified. As such, it is currently unknown whether continued translation in cardiovirusinfected cells depends on the catalytic activity of $3 \mathrm{C}^{\text {pro }}$, or whether cardioviruses employ a strategy that is perhaps more similar to that of aichivirus and mouse kobuvirus.

\section{Connection between nucleocytoplasmic trafficking disorder and impaired RLR signaling?}

In chapter 5, we studied whether and how enterovirus proteases affect RLR signaling. We showed that enterovirus $2 \mathrm{~A}^{\text {pro }}$, via its catalytic activity, suppresses RLR signaling. $2 \mathrm{~A}^{\text {pro }}$ is known to cleave RLR signaling proteins $(6,60)$, but a causal relationship between the cleavage of RLR signaling proteins and the suppressed induction of IFN- $\beta$ mRNA remains to be demonstrated. We showed that $2 \mathrm{~A}^{\text {pro's }}$ catalytic activity is needed to suppress IFN- $\beta$ mRNA, but we did not specify whether $2 \mathrm{~A}^{\text {pro's }}$ catalytic activity towards RLR signaling proteins is a contributing factor (chapter 5). While future research will undoubtedly aim to demonstrate a causal relationship between cleavage of RLR signaling proteins and suppression of IFN- $\alpha / \beta$ mRNA induction, we cannot exclude the contribution of alternative mechanisms at this point.
One alternative mechanism involves the ability of $2 \mathrm{~A}^{\text {pro }}$ to induce a so-called nucleocytoplasmic trafficking disorder (NCTD). $2 \mathrm{~A}^{\text {pro }}$ of several different enteroviruses cleaves nucleoporins, which results in the collapse of nuclear pores (63-68). As a result, active nucleocytoplasmic transport is disturbed and proteins can migrate in and out the nucleus via passive diffusion. Cardiovirus $L$ also suppresses the induction of IFN- $\alpha / \beta$ mRNA and it also induces NCTD, albeit via a different underlying molecular mechanism. L interacts with RAN-GTPase (69) to localize close to the nuclear envelope, where it facilitates the phosphorylation of nucleoporins (NUPs) $(70,71)$. The resulting negative charge on the NUPs destabilizes the nuclear pore complex and results in impaired functioning of the nuclear pore. It was previously demonstrated that MAPK kinase inhibitors inhibit NUP hyperphosphorylation, implicating cellular MAPK kinases in this phenomenon (72). L was recently reported to interact with, and prevent the inactivation of the cellular p9oribosomal S6 kinase (RSK) (73). RSK belongs to the MAPK family of kinases and thus is likely the kinase that phosphorylates the nucleoporins.

Collapse of the nuclear pore and the subsequent inhibition of active transport in and out of the nucleus can affect the regulated translocation of IRF3, IRF7 and NFKB and can thereby impair the transcription of IFN- $\alpha / \beta$ mRNA. Strikingly, the NCTD that is triggered by cardiovirus L did not prevent IRF3 from localizing to the nucleus (74). However, IRF3 lacking its nuclear translocation signal (NLS) also localized to the nucleus of cardiovirus-infected cells, while this IRF3- $\triangle$ NLS did not localize to the nucleus upon poly(I:C) stimulation (74), suggesting that nuclear localization of IRF3 observed during infection is due to the NCTD rather than its activation. Similarly, enterovirus $2 \mathrm{~A}^{\text {pro }}$ induced the relocalization of GFPNLS as well as well-known nuclear proteins (i.e. Sam68, nucleolin and La autoantigen) to the cytosol $(68,75)$. Enterovirus $2 \mathrm{~A}^{\text {pro }}$ and cardiovirus $\mathrm{L}$ were also shown to have a general effect on regulated nuclear transport; expression of either security protein interfered with overall nuclear import (by affecting the transportin 1 , transportin 3 and importin- $\alpha / \beta$ pathways) as well as nuclear export $(76,77)$. It is worth emphasizing that the translocation of IRF3, NFKB and STAT1 to the nucleus depends on the importin- $\alpha / \beta$ transporters (7880). Other viruses (i.e. hepatitis $C$ virus and Ebola) are known to counteract IFN- $\alpha / \beta$ and ISG expression by inhibiting the importin- $\alpha / \beta$-dependent nuclear import pathways ( 78 , 81). These collective observations strongly suggest that NCTD induction by cardiovirus $L$ and enterovirus $2 \mathrm{~A}^{\text {pro }}$ could affect the nuclear import of IRF3, IRF7 and NFKB and thus contribute to the suppression of IFN- $\alpha / \beta$ expression.

The effect of an impairment in nucleocytoplasmic transport on innate antiviral signaling has also been studied outside of the context of viral infection. It was recently demonstrated that NUP93 deficient cells have reduced expression of IFN- $\beta$ and the pro-inflammatory cytokine CXCL10 (82). NUP93 is essential for the formation of the nuclear pore complex. NUP93s 
C-terminal part stabilizes the initial NUP53-NUP155 interaction and thereby contributes to the structural formation of a pore in the nuclear membrane. Meanwhile, its N-terminal domain recruits NUP62 and thereby transforms the structural pore into a functional NPC that facilitates nuclear import and export (83). In NUP93 deficient cells, IRF3 failed to translocate to the nucleus and activation of both IRF3 and TBK1 was impaired (82), suggesting that nuclear pore integrity can influence the RLR signaling cascade upstream of IRF3 translocation (i.e. TBK1 and IRF3 phosphorylation). Cardiovirus $L$ as well as enterovirus $2 \mathrm{~A}^{\text {pro }}$ target NUP62, the NUP that interacts with NUP93 to facilitate transport through the NPC. Both enterovirus $2 \mathrm{~A}^{\text {pro }}$ and cardiovirus L were shown to reduce IRF3 phosphorylation and dimerization $(6-8,60,84$, 85). The observation that NUP93 depletion and the resulting disruption of the nuclear pore has an effect on the activation of both IRF3 and TBK1 could further support a role of NCTD in the suppression of IFN- $\alpha / \beta$ mRNA expression by cardiovirus $L$ and enterovirus $2 \mathrm{~A}^{\text {pro }}$.

To confirm a causal relationship between NCTD and suppression of IFN- $\alpha / \beta$ mRNA expression, one would need to use CRISPR-cas9 technology to generate NCTD-resistant cells, i.e. cells where all $2 \mathrm{~A}^{\text {pro }}$ cleavage sites in NUPs are mutated or cells where cardiovirus $\mathrm{L}$ can no longer induce hyperphosphorylation of NUPs. Unfortunately, the generation of such cell lines remains impossible. $2 \mathrm{~A}^{\text {pro }}$ cleaves multiple NUPs and a complete understanding of which NUPs are cleaved at which site is currently lacking. The NUP cleavage sites are best described for HRV2 2A $A^{\text {pro, }}$, which cleaves NUP62 at six different positions (A103, G177, G201, G218, G247, A298) and NUP98 at two different positions (G374 and G552) $(64,66,86)$, but the cleavage site(s) in NUP153 $(66,76)$ remain to be elucidated. Moreover, $2 \mathrm{~A}^{\text {pro }}$ may cleave other NUPs as well, thereby hindering the generation of cells that are completely resistant to NCTD induction. The generation of cells resistant against cardio L-induced NCTD would be equally challenging. The hyperphosphorylation of NUPs that occurs during cardiovirus infection, which depends on L, was suggested to be analogous to NUP phosphorylation during prophase, the first stage of mitosis (70). Mutation of phosphorylation sites that possibly overlap with those normally used during cell division will most likely not result in a viable cell line. In conclusion, although the CRISPR-cas9 technology provides an exciting new horizon where it is possible to introduce mutations in endogenous genes, the generation of NCTDresistant cell lines remains impossible for a variety of reasons.

Taken together, it remains to be elucidated exactly how enterovirus $2 \mathrm{~A}^{\text {pro }}$ and cardiovirus $\mathrm{L}$ suppress RLR signaling. As discussed above there are strong indications that both security proteins rely on NCTD to inhibit the expression of IFN- $\alpha / \beta$, but unfortunately it remains difficult to design the experiments needed to study this hypothesis. $2 \mathrm{~A}^{\text {pro }}$ is known to cleave the RLR signaling proteins MDA5 and MAVS, while RLR signaling proteins remain intact

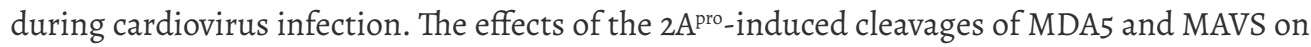
IFN- $\alpha / \beta$ mRNA induction remain to be demonstrated, but cleavage of RLR signaling proteins is commonly considered a strategy for viruses to counteract the expression of IFN- $\alpha / \beta$. The timing of $2 \mathrm{~A}^{\text {pro }}$ induced NCTD and $2 \mathrm{~A}^{\text {pro }}$ induced cleavage of RLR signaling proteins varies. NCTD is observed as early as 2 hours post infection $(63,67)$, while MDA5 and MAVS cleavage by enterovirus $2 \mathrm{~A}^{\text {pro }}$ is first detectable at $6-8$ hours post infection $(6,60)$. The fast induction of NCTD could be considered as an additional argument that NCTD may be the main contributor to the early suppression of IFN- $\alpha / \beta$ expression. The cleavage of RLR proteins by enterovirus $2 \mathrm{~A}^{\text {pro }}$ may well be a contributing factor to the continued suppression of IFN- $\alpha / \beta$ expression during the mid-to-late phase of enterovirus infection. Of note, cardiovirus infection does not result in cleavage of RLR proteins. Whether cardiovirus L utilizes additional mechanisms to suppress IFN- $\alpha / \beta$ expression during the mid-to-late phase of infection, remains to be established.

\section{What is the role of the deISGylase activity of FMDV $\mathrm{L}^{\text {pro? }}$}

FMDV L pro does not induce NCTD, yet it suppresses the induction of IFN- $\alpha / \beta$ (59). In chapter 4 , we studied how FMDV L $^{\text {pro }}$ suppresses RLR signaling and identified a correlation between the ability of $L^{\text {pro }}$ to cleave RLR signaling proteins and $L^{\text {pro's }}$ ability to suppress IFN- $\beta$ mRNA induction. $\mathrm{L}^{\text {pro }}$ also possesses deISGylase and, to a lesser extent, deubiquitinase (DUB) activity (chapter 3 and (3)) and these enzymatic activities are used by other viruses to suppress RLR signaling. In particular, the nidoviruses are known to suppress IFN- $\alpha / \beta$ transcription via the DUB/deISGylase activity of their papain-like protease $(87,88)$. It was suggested that $\mathrm{L}^{\text {pro's }}$ DUB activity similarly contributes to suppression of RLR signaling (3). However, in chapter 3 we found no evidence of significant DUB activity of $L^{\text {pro }}$ in infected cells, and in chapter 4 we suggest that FMDV $L^{\text {pro }}$ suppresses IFN- $\alpha / \beta$ transcription independent from its deISGylase activity.

Our data demonstrate that $\mathrm{L}^{\text {pro }}$ can suppress IFN- $\beta$ transcription and suggest that this ability relies on $\mathrm{L}^{\text {pro's }}$ capacity to cleave RLR signaling proteins rather than its deISGylase activity, although a true causal relationship remains to be demonstrated. A recent report demonstrates that the deISGylase activity of $L^{\text {pro }}$ contributes to viral spread in vivo (89). In agreement with our findings, impaired deISGylase activity of $\mathrm{L}^{\text {pro }}$ did not result in an increase in IFN- $\alpha / \beta$ or ISG mRNA in this study. How $L^{\text {pro' }}$ deISGylase activity benefits FMDV infection, both in vivo and in cellulo, is currently unknown. Our work has elucidated residues in $\mathrm{L}^{\text {pro }}$ that are essential for either its ability to cleave RLR signaling proteins or its deISGylase activity. Introduction of mutations in $L^{\text {pro }}$ in the FMDV genome is feasible provided that $\mathrm{L}^{\mathrm{pro}}$ can release itself from the polyprotein. This is illustrated by the previous successful generation of an FMDV strain carrying mutations in the SAP domain and an FMDV strain carrying a mutation affecting $\mathrm{L}^{\text {pro's }}$ deISGylase activity $(89,90)$. This provides an opportunity to introduce the mutations we identified into the FMDV genome to study how $\mathrm{L}^{\text {pro's }}$ deISGylase activity contributes to viral spread, possibly in an in vivo setting in livestock animals. Thus far, all data suggest that $\mathrm{L}^{\text {pro's }}$ deISGylase activity may be needed 
to combat one or several of the many other antiviral functions of ISG15 (reviewed in (91)), rather than contributing to the suppression of RLR signaling and thus the suppression of IFN- $\alpha / \beta$ expression.

In chapter 3, we also demonstrated that $\mathrm{L}^{\text {pro }}$ is a unique deISGylase, leaving a diglycylatedlysine on its substrates. The presence of these diglycylated lysines provides an interesting opportunity to investigate whether and how ISG15 regulates antiviral signaling. A specific monoclonal antibody suitable to enrich diglycylated lysine-containing peptides and proteins is available $(92,93)$. Previous applications of this antibody have been in the enrichment of diglycyl-lysine containing peptides in mass spectrometry analysis of ubiquitinated proteins, as tryptic digestion of ubiquitinated and ISGylated proteins results in the same diglycylated-lysine residues. A major drawback of these procedures has been the relatively late enrichment of modified peptides. There is the risk of missing modified proteins when the ubiquitin or ISG15 is lost during sample preparation. Additionally, all prior enrichment steps precipitate the total pool of a substrate protein, while only the small percentage of ubiquitin or ISG15-modified protein is of interest for the MS analysis. Expressing $L^{\text {pro }}$ in

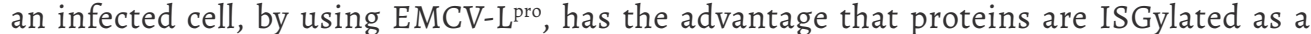
result of infection and the fragile ubiquitin- or ISG-modification is transformed into a stable diglycylated-lysine residue before cell lysis. Furthermore, we can directly enrich for those peptides carrying diglycylated-lysines, which will greatly improve the detection of modified proteins. This systematic approach will inform us of the (signaling) proteins that are modified with ISG15 upon viral infection, which has the potential to greatly increase our understanding of the function of protein ISGylation during infection.

\section{Concluding remarks}

In this thesis we studied how different picornavirus security proteins suppress or circumvent the activation of antiviral host responses. In the experimental chapters we provided new insight into the mechanisms used by FMDV, human enteroviruses and aichivirus to evade the induction of type I interferon or the antiviral properties of the cellular stress response. Here, we summarized our main findings and discussed commonly found tactics employed by different picornaviruses and their different security proteins. The analysis of the different mechanisms used to evade the antiviral responses revealed the possibility that multiple effects could be linked to a single action of a security protein. For example, the cleavage of eIF4G is known to ensure shut-off of cap-dependent (host) translation. Additionally, evidence is emerging that targeting of eIF4G could play a central role in the mechanisms used by security proteins to counteract SG formation. Similarly, the induction of NCTD by security proteins could have a direct effect on the RLR signaling pathway and thus on the expression of type I interferon and additional pro-inflammatory cytokines. The "network" of the different effector functions of a security protein, and whether and how these effects can be linked or traced back to a single activity (e.g. eIF4G cleavage or inducing NCTD), is largely unknown. Further research into these networks may allow us to fully understand how (picorna)viruses evade antiviral host responses, something that is of vital importance for in vivo infection and thus may provide new insights that can be used in vaccine development. 


\section{References}

1. de Los Santos T, Diaz-San Segundo F, Grubman MJ. 2007. Degradation of nuclear factor kappa B during footand-mouth disease virus infection. J Virol 81:12803-12815.

2. Wang D, Fang L, Luo R, Ye R, Fang Y, Xie L, Chen H, Xiao S. 2010. Foot-and-mouth disease virus leader proteinase inhibits dsRNA-induced type I interferon transcription by decreasing interferon regulatory factor 3/7 in protein levels. Biochem Biophys Res Commun 399:72-78.

3. Wang D, Fang L, Li P, Sun L, Fan J, Zhang Q, Luo R, Liu X, Li K, Chen H, Chen Z, Xiao S. 2011. The Leader Proteinase of Foot-and-Mouth Disease Virus Negatively Regulates the Type I Interferon Pathway by Acting as a Viral Deubiquitinase. J Virol 85:3758-3766.

4. Onomoto K, Jogi M, Yoo JS, Narita R, Morimoto S, Takemura A, Sambhara S, Kawaguchi A, Osari S, Nagata K, Matsumiya T, Namiki H, Yoneyama M, Fujita T. 2012. Critical role of an antiviral stress granule containing RIG-I and PKR in viral detection and innate immunity. PLoS One 7:e43031.

5. NgCS,Jogi M, YooJ-S, OnomotoK, Koike S, Iwasaki T, Yoneyama M, Kato H, Fujita T. 2013. Encephalomyocarditis virus disrupts stress granules, the critical platform for triggering antiviral innate immune responses. J Virol 87:9511-22.

6. Feng Q, Langereis MA, Lork M, Nguyen M, Hato S V, Lanke K, Emdad L, Bhoopathi P, Fisher PB, Lloyd RE, van Kuppeveld FJM. 2014. Enterovirus 2Apro targets MDA5 and MAVS in infected cells. J Virol 88:3369-78.

7. Hato S V, Ricour C, Schulte BM, Lanke KHW, de Bruijn M, Zoll J, Melchers WJG, Michiels T, van Kuppeveld FJM. 2007. The mengovirus leader protein blocks interferon-alpha/beta gene transcription and inhibits activation of interferon regulatory factor 3. Cell Microbiol 9:2921-2930.

8. Stavrou S, Feng Z, Lemon SM, Roos RP. 2010. Different Strains of Theiler's Murine Encephalomyelitis Virus Antagonize Different Sites in the Type I Interferon Pathway. J Virol 75:7811-7817.

9. Rabouw HH, Langereis MA, Knaap RCM, Dalebout TJ, Canton J, Sola I, Enjuanes L, Bredenbeek PJ, Kikkert M, de Groot RJ, van Kuppeveld FJM. 2016. Middle East Respiratory Coronavirus Accessory Protein 4a Inhibits PKR-Mediated Antiviral Stress Responses. PLoS Pathog 12:e1005982.

10. Borghese F, Sorgeloos F, Cesaro T, Michiels T. 2019. The Leader Protein of Theiler's Virus Prevents the Activation of PKR. J Virol 93.

11. Borghese F, Michiels T. 2011. The Leader Protein of Cardioviruses Inhibits Stress Granule Assembly. J Virol 85:9614-9622.

12. White JP, Cardenas AM, Marissen WE, Lloyd RE. 2007. Inhibition of Cytoplasmic mRNA Stress Granule Formation by a Viral Proteinase. Cell Host Microbe 2:295-305.

13. Fung G, Ng CS, Zhang J, Shi J, Wong J, Piesik P, Han L, Chu F, Jagdeo J, Jan E, Fujita T, Luo H. 2013. Production of a dominant-negative fragment due to $\mathrm{G}_{3} \mathrm{BP} 1$ cleavage contributes to the disruption of mitochondriaassociated protective stress granules during CVB3 infection. PLoS One 8:e79546.

14. Zhang Y, Yao L, Xu X, Han H, Li P, Zou D, Li X, Zheng L, Cheng L, Shen Y, Wang X, Wu X, Xu J, Song B, Xu S, Zhang H, Cao H. 2018. Enterovirus 71 inhibits cytoplasmic stress granule formation during the late stage of infection. Virus Res 255:55-67.

15. Yang $X, H u$, Fan $S, Z$ Zhang $Q$, Zhong $Y$, Guo D, Qin Y, Chen M. 2018. Picornavirus $2 A$ protease regulates stress granule formation to facilitate viral translation. PLOS Pathog 14:e1006901.

16. Yang X, Hu Z, Zhang Q, Fan S, Zhong Y, Guo D, Qin Y, Chen M. 2019. SG formation relies on eIF4GI-G3BP interaction which is targeted by picornavirus stress antagonists. Cell Discov 5:1.

17. Low WK, Dang Y, Schneider-Poetsch T, Shi Z, Choi NS, Merrick WC, Romo D, Liu JO. 2005. Inhibition of eukaryotic translation initiation by the marine natural product pateamine A. Mol Cell.

18. Mazroui R, Sukarieh R, Bordeleau ME, Kaufman RJ, Northcote P, Tanaka J, Gallouzi I, Pelletier J. 2006. Inhibition of ribosome recruitment induces stress granule formation independently of eukaryotic initiation factor $2 \alpha$ phosphorylation. Mol Biol Cell.
19. Jain S, Wheeler JR, Walters RW, Agrawal A, Barsic A, Parker R. 2016. ATPase-Modulated Stress Granules Contain a Diverse Proteome and Substructure. Cell 164:487-98.

20. Khong A, Matheny T, Jain S, Mitchell SF, Wheeler JR, Parker R. 2017. The Stress Granule Transcriptome Reveals Principles of mRNA Accumulation in Stress Granules. Mol Cell 68:808-820.e5.

21. Yang P, Mathieu C, Kolaitis RM, Zhang P, Messing J, Yurtsever U, Yang Z, Wu J, Li Y, Pan Q, Yu J, Martin EW, Mittag T, Kim HJ, Taylor JP. 2020. G3BP1 Is a Tunable Switch that Triggers Phase Separation to Assemble Stress Granules. Cell 181:325-345.e28.

22. Kedersha N, Chen S, Gilks N, Li W, Miller IJ, Stahl J, Anderson P. 2002. Evidence that ternary complex (eIF2GTP-tRNAiMet)-Deficient preinitiation complexes are core constituents of mammalian stress granules. Mol Biol Cell 13:195-210.

23. Ferrero P V., Layana C, Paulucci E, Gutiérrez P, Hernández G, Rivera-Pomar R V. 2012. Cap bindingindependent recruitment of eIF4E to cytoplasmic foci. Biochim Biophys Acta - Mol Cell Res 1823:1217-1224.

24. Frydryskova K, Masek T, Borcin K, Mrvova S, Venturi V, Pospisek M. 2016. Distinct recruitment of human eIF4E isoforms to processing bodies and stress granules. BMC Mol Biol 17.

25. Gradi A, Foeger N, Strong R, Svitkin Y V., Sonenberg N, Skern T, Belsham GJ. 2004. Cleavage of Eukaryotic Translation Initiation Factor 4GII within Foot-and-Mouth Disease Virus-Infected Cells: Identification of the L-Protease Cleavage Site In Vitro. J Virol.

26. Dougherty JD, Tsai WC, Lloyd RE. 2015. Multiple poliovirus proteins repress cytoplasmic RNA granules. Viruses 7:6127-6140.

27. Diep J, Ooi YS, Wilkinson AW, Peters CE, Foy E, Johnson JR, Zengel J, Ding S, Weng KF, Laufman O, Jang G, Xu J, Young T, Verschueren E, Kobluk KJ, Elias JE, Sarnow P, Greenberg HB, Hüttenhain R, Nagamine CM, Andino R, Krogan NJ, Gozani O, Carette JE. 2019. Enterovirus pathogenesis requires the host methyltransferase SETD3. Nat Microbiol.

28. Groskreutz DJ, Babor EC, Monick MM, Varga SM, Hunninghake GW. 2010. Respiratory syncytial virus limits $\alpha$ subunit of eukaryotic translation initiation factor 2 (eIF2 $\alpha$ ) phosphorylation to maintain translation and viral replication. J Biol Chem.

29. Li Y, Zhang C, Chen X, Yu J, Wang Y, Yang Y, Du M, Jin H, Ma Y, He B, Cao Y. 2011. ICP34.5 protein of herpe simplex virus facilitates the initiation of protein translation by bridging eukaryotic initiation factor zalph (eIF2alpha) and protein phosphatase 1. J Biol Chem 286:24785-92.

30. Cruz JLG, Sola I, Becares M, Alberca B, Plana J, Enjuanes L, Zuñiga S. 2011. Coronavirus Gene 7 Counteracts Host Defenses and Modulates Virus Virulence. PLoS Pathog 7:101002090.

31. Brand SR, Kobayashi R, Mathews MB. 1997. The Tat protein of human immunodeficiency virus type 1 is a substrate and inhibitor of the interferon-induced, virally activated protein kinase, PKR. J Biol Chem.

32. Garcia-Moreno M, Sanz MA, Carrasco L. 2015. Initiation codon selection is accomplished by a scanning mechanism without crucial initiation factors in sindbis virus subgenomic mRNA. RNA.

33. Sanz MÁ, Castelló A, Ventoso I, Berlanga IJ, Carrasco L. 2009. Dual mechanism for the translation of subgenomic mRNA from Sindbis virus in infected and uninfected cells. PLoS One.

34. Gorchakov R, Frolova E, Williams BRG, Rice CM, Frolov I. 2004. PKR-Dependent and -Independent Mechanisms Are Involved in Translational Shutoff during Sindbis Virus Infection. J Virol.

35. Ventoso I, Sanz MA, Molina S, Berlanga JJ, Carrasco L, Esteban M. 2006. Translational resistance of late alphavirus mRNA to eIF2 $\alpha$ phosphorylation: A strategy to overcome the antiviral effect of protein kinase PKR. Genes Dev.

36. Jan E, Sarnow P. 2002. Factorless ribosome assembly on the internal ribosome entry site of cricket paralysis virus. J Mol Biol.

37. Wilson JE, Pestova T V., Hellen CUT, Sarnow P. 2000. Initiation of protein synthesis from the A site of the ribosome. Cell.

38. Pestova T V., Hellen CUT. 2003. Translation elongation after assembly of ribosomes on the Cricket paralysis virus internal ribosomal entry site without initiation factors or initiator tRNA. Genes Dev. 
39. Sweeney TR, Abaeva IS, Pestova T V., Hellen CUT. 2014. The mechanism of translation initiation on type 1 picornavirus IRESs. EMBO J.

40. Lozano G, Martínez-Salas E. 2015. Structural insights into viral IRES-dependent translation mechanisms. Curr Opin Virol 12:113-120.

41. Yu Y, Sweeney TR, Kafasla P, Jackson RJ, Pestova T V., Hellen CUT. 2011. The mechanism of translation initiation on Aichivirus RNA mediated by a novel type of picornavirus IRES. EMBO J.

42. Pestova T V, Hellen CU, Shatsky IN. 1996. Canonical eukaryotic initiation factors determine initiation of translation by internal ribosomal entry. Mol Cell Biol.

43. Redondo N, Sanz MA, Welnowska E, Carrasco L. 2011. Translation without eIF2 Promoted by Poliovirus 2A Protease. PLoS One 6:e25699.

44. Black TL, Safer B, Hovanessian A, Katze MG. 1989. The cellular 68,000-Mr protein kinase is highly autophosphorylated and activated yet significantly degraded during poliovirus infection: implications for translational regulation. J Virol 63:2244-51.

45. White JP, Reineke LC, Lloyd RE. 2011. Poliovirus Switches to an eIF2-Independent Mode of Translation during Infection. J Virol 85:8884-8893.

46. de Breyne S, Bonderoff JM, Chumakov KM, Lloyd RE, Hellen CUT. 2008. Cleavage of eukaryotic initiation factor eIF5 $B$ by enterovirus $3 \mathrm{C}$ proteases. Virology.

47. Guillon L, Schmitt E, Blanquet S, Mechulam Y. 2005. Initiator tRNA binding by e/aIF5B, the eukaryotic/ archaeal homologue of bacterial initiation factor IF2. Biochemistry.

48. Terenin IM, Dmitriev SE, Andreev DE, Shatsky IN. 2008. Eukaryotic translation initiation machinery can operate in a bacterial-like mode without elF2. Nat Struct Mol Biol.

49. Pestova T V., De Breyne S, Pisarev A V., Abaeva IS, Hellen CUT. 2008. eIF2-dependent and eIF2-independent modes of initiation on the CSFV IRES: A common role of domain II. EMBO J.

50. Kieft JS, Zhou K, Jubin R, Doudna JA. 2001. Mechanism of ribosome recruitment by hepatitis C IRES RNA. RNA.

51. Pestova T V., Shatsky IN, Fletcher SP, Jackson RJ, Hellen CUT. 1998. A prokaryotic-like mode of cytoplasmic eukaryotic ribosome binding to the initiation codon during internal translation initation of hepatitis $\mathrm{C}$ and classical swine fever virus RNAs. Genes Dev.

52. Sizova D V., Kolupaeva VG, Pestova T V., Shatsky IN, Hellen CUT. 1998. Specific Interaction of Eukaryotic Translation Initiation Factor 3 with the 5' Nontranslated Regions of Hepatitis C Virus and Classical Swine Fever Virus RNAs. J Virol.

53. Otto GA, Puglisi JD. 2004. The pathway of HCV IRES-mediated translation initiation. Cell.

54. Ji H, Fraser CS, Yu Y, Leary J, Doudna JA. 2004. Coordinated assembly of human translation initiation complexes by the hepatitis C virus internal ribosome entry site RNA. Proc Natl Acad Sci U S A.

55. Moral-López P, Alvarez E, Redondo N, Skern T, Carrasco L. 2014. L protease from foot and mouth disease virus confers eIF2-independent translation for mRNAs bearing picornavirus IRES. FEBS Lett 588:4053-4059.

56. Haghighat A, Svitkin Y, Novoa I, Kuechler E, Skern T, Sonenberg N. 1996. The eIF4G-eIF4E complex is the target for direct cleavage by the rhinovirus $2 \mathrm{~A}$ proteinase. J Virol.

57. Devaney MA, Vakharia VN, Lloyd RE, Ehrenfeld E, Grubman MJ. 1988. Leader protein of foot-and-mouth disease virus is required for cleavage of the p220 component of the cap-binding protein complex. J Virol 62:4407-9.

58. Ventoso I, MacMillan SE, Hershey JW b., Carrasco L. 1998. Poliovirus 2A proteinase cleaves directly the eIF4G subunit of eIF-4F complex. FEBS Lett 435:79-83.

59. de Los Santos T, de Avila Botton S, Weiblen R, Grubman MJ. 2006. The leader proteinase of foot-and-mouth disease virus inhibits the induction of beta interferon $\mathrm{mRNA}$ and blocks the host innate immune response. J Virol 80:1906-14.

60. Wang B, Xi X, Lei X, Zhang X, Cui S, Wang J, Jin Q, Zhao Z. 2013. Enterovirus 71 Protease 2Apro Targets MAVS to Inhibit Anti-Viral Type I Interferon Responses. PLoS Pathog 9:e1003231.
61. Welnowska E, Sanz MA, Redondo N, Carrasco L. 2011. Translation of viral mRNA without active eIF2: The case of picornaviruses. PLoS One.

62. Rabouw HH, Visser LJ, Passchier TC, Langereis MA, Liu F, Giansant P, van Vliet AL, Dekker JG, van der Grein SG, Saucedo JG, Anand AA, Trellet ME, Bonvin AMJJ, Walter P, Heck AJR, Groot RJ de, van Kuppeveld FJM. 2020. Inhibition of the integrated-stress-response by viral proteins that block p-eIF2•eIF2B association. Nat Microbiol (accepted for publication).

63. Belov GA, Lidsky P V, Mikitas O V, Egger D, Lukyanov KA, Bienz K, Agol VI. 2004. Bidirectional increase in permeability of nuclear envelope upon poliovirus infection and accompanying alterations of nuclear pores. I Virol 78:10166-77.

64. Park N, Skern T, Gustin KE. 2010. Specific cleavage of the nuclear pore complex protein Nup62 by a viral protease. J Biol Chem 285:28796-805.

65. Park N, Katikaneni P, Skern T, Gustin KE. 2008. Differential targeting of nuclear pore complex proteins in poliovirus-infected cells. J Virol 82:1647-55

66. Watters K, Palmenberg AC. 2011. Differential processing of nuclear pore complex proteins by rhinovirus $2 \mathrm{~A}$ proteases from different species and serotypes. J Virol 85:10874-83.

67. Gustin KE, Sarnow P. 2001. Effects of poliovirus infection on nucleo-cytoplasmic trafficking and nuclear pore complex composition. EMBO J 20:240-9.

68. Gustin KE, Sarnow P. 2002. Inhibition of Nuclear Import and Alteration of Nuclear Pore Complex Composition by Rhinovirus. J Virol.

69. Porter FW, Bochkov YA, Albee AJ, Wiese C, Palmenberg AC. 2006. A picornavirus protein interacts with RanGTPase and disrupts nucleocytoplasmic transport. Proc Natl Acad Sci U S A 103:12417-22.

70. Bardina M V, Lidsky P V, Sheval E V, Fominykh K V, van Kuppeveld FJM, Polyakov VY, Agol VI. 2009. Mengovirus-induced rearrangement of the nuclear pore complex: hijacking cellular phosphorylation machinery. J Virol 83:3150-61.

71. Porter FW, Palmenberg AC. 2009. Leader-induced phosphorylation of nucleoporins correlates with nuclear trafficking inhibition by cardioviruses. J Virol 83:1941-51.

72. Porter FW, Brown B, Palmenberg AC. 2010. Nucleoporin Phosphorylation Triggered by the Encephalomyocarditis Virus Leader Protein Is Mediated by Mitogen-Activated Protein Kinases. J Virol.

73. Sorgeloos F, Peeters M, Michiels T. 2018. Convergent evolution of viruses and bacteria to hijack host cell kinases, p. 55. In Europic conference 2018 Abstract O49.

74. Delhaye S, van Pesch V, Michiels T. 2004. The Leader Protein of Theiler's Virus Interferes with Nucleocytoplasmic Trafficking of Cellular Proteins. J Virol 78:4357-62.

75. Gustin KE, Sarnow P. 2001. Effects of poliovirus infection on nucleo-cytoplasmic trafficking and nuclear pore complex composition. EMBO J.

76. Watters K, Inankur B, Gardiner JC, Warrick J, Sherer NM, Yin J, Palmenberg AC. 2017. Differential Disruption of Nucleocytoplasmic Trafficking Pathways by Rhinovirus 2A Proteases. J Virol 91:e02472-16.

77. Ciomperlik JJ, Basta HA, Palmenberg AC. 2015. Three cardiovirus Leader proteins equivalently inhibit four different nucleocytoplasmic trafficking pathways. Virology.

78. Gagné B, Tremblay N, Park AY, Baril M, Lamarre D. 2017. Importin $\beta 1$ targeting by hepatitis C virus NS3/4A protein restricts IRF 3 and NF- $x$ B signaling of IFNBi antiviral response. Traffic 18:362-377.

79. Stelma T, Leaner VD. 2017. KPNB1-mediated nuclear import is required for motility and inflammatory transcription factor activity in cervical cancer cells. Oncotarget.

80. Fagerlund R, Mélen K, Kinnunen L, Julkunen I. 2002. Arginine/lysine-rich nuclear localization signals mediate interactions between dimeric STATs and importin alpha 5. J Biol Chem.

81. Reid SP, Leung LW, Hartman AL, Martinez O, Shaw ML, Carbonnelle C, Volchkov VE, Nichol ST, Basler CF. 2006. Ebola Virus VP24 Binds Karyopherin 1 and Blocks STAT1 Nuclear Accumulation. J Virol 80:5156-5167.

82. Monwan W, Kawasaki T, Hasan MZ, Ori D, Kawai T. 2019. Identification of nucleoporin 93 (Nup93) that mediates antiviral innate immune responses. Biochem Biophys Res Commun. 
83. Sachdev R, Sieverding C, Flötenmeyer M, Antonin W. 2012. The C-terminal domain of Nup93 is essential for assembly of the structural backbone of nuclear pore complexes. Mol Biol Cell.

84. Drahos J, Racaniello VR. 2009. Cleavage of IPS-1 in cells infected with human rhinovirus. J Virol 83:11581-7.

85. Mukherjee A, Morosky SA, Delorme-Axford E, Dybdahl-Sissoko N, Oberste MS, Wang T, Coyne CB. 2011. The coxsackievirus B 3Cpro protease cleaves MAVS and TRIF to attenuate host type I interferon and apoptotic signaling. PLoS Pathog 7:e1001311.

86. Park N, Schweers NJ, Gustin KE. 2015. Selective Removal of FG Repeat Domains from the Nuclear Pore Complex by Enterovirus 2A pro .J Virol 89:11069-11079.

87. Bailey-Elkin BA, Knaap RCM, Johnson GG, Dalebout TJ, Ninaber DK, van Kasteren PB, Bredenbeek PJ, Snijder EJ, Kikkert M, Mark BL. 2014. Crystal structure of the Middle East respiratory syndrome coronavirus (MERS-CoV) papain-like protease bound to ubiquitin facilitates targeted disruption of deubiquitinating activity to demonstrate its role in innate immune suppression. J Biol Chem 289:34667-82.

88. van Kasteren PB, Knaap RCM, van den Elzen P, Snijder EJ, Balasuriya UBR, van den Born E, Kikkert M. 2015. In vivo assessment of equine arteritis virus vaccine improvement by disabling the deubiquitinase activity of papain-like protease 2. Vet Microbiol 178:132-7.

89. Medina GN, Azzinaro P, Ramirez-Medina E, Gutkoska J, Fang Y, Diaz-San Segundo F, de los Santos T. 2020. Impairment of the deISGylation activity of FMDV Lpro causes attenuation in vitro and in vivo. J Virol ePub ahead of print.

90. de los Santos T, Diaz-San Segundo F, Zhu J, Koster M, Dias CCA, Grubman MJ. 2008. A Conserved Domain in the Leader Proteinase of Foot-and-Mouth Disease Virus Is Required for Proper Subcellular Localization and Function. J Virol 83:1800-10.

91. Dzimianski J V., Scholte FEM, Bergeron E, Pegan SD. 2019. ISG15: it's Complicated. J Mol Biol Sooz22836:30136-6.

92. Kim W, Bennett EJ, Huttlin EL, Guo A, Li J, Possemato A, Sowa ME, Rad R, Rush J, Comb MJ, Harper JW, Gygi SP. 2011. Systematic and quantitative assessment of the ubiquitin-modified proteome. Mol Cell.

93. Wagner SA, Beli P, Weinert BT, Nielsen ML, Cox J, Mann M, Choudhary C. 2011. A Proteome-wide, Quantitative Survey of In Vivo Ubiquitylation Sites Reveals Widespread Regulatory Roles. Mol Cell Proteomics. 


\title{
Chapter 8
}

\author{
Addendum
}

8 


\section{Nederlandse samenvatting}

De picornavirussen vormen een grote virusfamilie waartoe enkele bekende humane en veterinaire virussen behoren. Bekende picornavirussen zijn onder andere poliovirus, hepatitis A virus en het mond-en-klauwzeervirus. Het mond-en-klauwzeervirus was zelfs het eerste dierlijke virus - een infectieus agens dat kleiner is dan een bacterie maar wel ziekte kan overdragen - dat werd geïdentificeerd. Kort na de ontdekking van het monden-klauwzeervirus in 1898, werd in 1908 het poliovirus, een picornavirus dat mensen kan infecteren, geïdentificeerd. De term "virus", afgeleid van het Latijnse woord voor vergif, werd geïntroduceerd om naar deze ziekteverwekkers te verwijzen. Het was destijds echter volstrekt onbekend wat deze ziekteverwekkers waren of hoe ze ziekte kunnen veroorzaken. Sinds hun ontdekking zijn deze twee virussen intensief bestudeerd. Onder andere dit onderzoek was een van drijfveren achter de ontwikkeling van de moleculaire virologie als zelfstandig vakgebied. Daarnaast heeft het onderzoek naar de vermeerdering van deze virussen, welke ten koste gaat van hun gastheer, bijgedragen aan onze kennis van de moleculaire processen in de cel.

Virussen zijn acellulaire parasieten die voor hun vermeerdering afhankelijk zijn van het metabolisme van hun gastheer. De vermeerdering van alle virussen, en dus ook van picornavirussen, kan worden herkend door de gastheer. De gastheer kan vervolgens enkele antivirale responsen inschakelen om de vermenigvuldiging en verspreiding van het virus tegen te gaan. Voor een succesvolle infectie is het virus genoodzaakt om deze antivirale responsen te onderdrukken of te ontduiken. Het onderzoek beschreven in dit proefschrift richt zich op de manieren waarop enkele picornavirussen de verschillende antivirale responsen onderdrukken of ontduiken. Hierbij wordt specifiek gekeken naar twee vroege antivirale reacties van een geïnfecteerde cel; de expressie van type I-interferon en het inschakelen van de cellulaire stressrespons.

De type I-interferon eiwitten (IFN- $\alpha / \beta$ ) zijn centrale spelers in het aangeboren immuunsysteem. Deze pro-inflammatoire cytokinen vormen de eerste alarmsignalen en staan aan de basis van de verdere immuunrespons. Virusreplicatie kan worden gedetecteerd door de familie van RIG-I-like receptoren (RLRs). Dit leidt tot het inschakelen van de zogenaamde RLR-signaaltransductieroute, wat resulteert in de expressie van IFN- $\alpha / \beta$. Het geproduceerde IFN- $\alpha / \beta$ kan vervolgens aan de interferonreceptor binden en zorgt voor de expressie van honderden interferon-geïnduceerde genen, waardoor deze cellen in een verdedigingsmodus, de antivirale staat, komen. Dit gebeurt op zowel de geïnfecteerde cel als op niet-geïnfecteerde omliggende cellen waardoor de ongeïnfecteerde cellen beschermd zijn. Daardoor heeft expressie van IFN- $\alpha / \beta$ een groot inperkend effect op de verspreiding van het virus. Logischerwijs hebben virussen effectieve methoden ontwikkeld om de nadelige gevolgen van IFN- $\alpha / \beta$ expressie te voorkomen. Naast het voorkomen van IFN- $\alpha / \beta$ expressie, kunnen virussen ook voorkomen dat interferon-geïnduceerde genen tot expressie komen. Deze inteferon-geïnduceerde genen hebben veelal een antivirale werking en hun expressie wordt geïnduceerd door IFN- $\alpha / \beta$.

Detectie van virusreplicatie kan ook resulteren in het inschakelen van de cellulaire stressrespons. Dit heeft tot gevolg dat eiwitproductie (de translatie) in de cel wordt onderdrukt dankzij de fosforylering van translatieinitiatiefactor 2 (eIF2), met mogelijke negatieve effecten voor de productie van nieuwe virussen. De onderdrukte translatie heeft daarnaast tot gevolg dat de overgebleven mRNA moleculen door de cel verzameld worden in zogenaamde 'stress granules', aggregaten van mRNA en translatie factoren. Vanuit de stress granules kan de translatie eventueel weer opgestart worden, nadat de cel de stresssituatie heeft opgelost. Van deze stress granules wordt gezegd dat ze een antivirale werking hebben, bijvoorbeeld doordat ze zouden functioneren als signaleringsplatform voor antivirale signaaltransductieroutes. Virussen hebben verschillende mechanismen ontwikkeld om de cellulaire stressrespons te onderdrukken of te ontwijken. Hierbij kan grofweg een onderscheid worden gemaakt tussen het voorkomen of ongedaan maken van de fosforylering van eIF2 om de virale translatie te bevorderen, en het tegengaan van de vorming van stress granules.

Van verschillende picornavirussen is bekend dat ze voor het onderdrukken of ontwijken van deze twee antivirale responsen gebruik maken van hun "security proteins", virale eiwitten die niet strikt noodzakelijk zijn voor de vermenigvuldiging van het virus, maar wel een positief effect hebben op de verspreiding van het virus. In dit proefschrift beschrijven we onderzoek naar de exacte mechanismen waarmee enkele picornavirus security proteins de genoemde antivirale responsen onderdrukken of ontwijken.

In hoofdstuk 2-4, onderzochten we het mond-en-klauwzeervirus. Voor dit virus was nog weinig bekend omtrent het onderdrukken van de cellulaire stressrespons. In hoofdstuk 2 beschrijven we dat dit virus de vorming van stress granules onderdrukt via het leader eiwit. We laten zien dat een mond-en-klauwzeervirus zonder dit leader eiwit de stress granule-vorming niet langer kan onderdrukken. Het mond-en-klauwzeer leader eiwit is een protease, daarom hebben we onderzocht of leader specifieke stress granule eiwitten kan knippen. Dit bleek het geval, leader knipt de eiwitten G3BP1 en G3BP2.

Het leader protease van mond-en-klauwzeervirus is ook bekend vanwege zijn rol in de onderdrukking van type I-interferon, al is het onderliggende mechanisme niet volledig opgehelderd. De RLR-signaaltransductieroute wordt gereguleerd via de post-translationele modificaties fosforylering en ubiquitinering. Het is bekend dat verschillende virale 
proteases door middel van deubiquitinase activiteit een negatief effect hebben op de RLRsignaaltransductie route. Dit mechanisme is ook voorgesteld voor het leader protease van mond-en-klauwzeervirus, al is dit voornamelijk gebaseerd op bevindingen in cellen waarin ubiquitine en leader tot expressie zijn gebracht, en dus niet op data uit geïnfecteerde cellen. In hoofdstuk 3 beschrijven wij dat leader voornamelijk deISGylase activiteit bezit en slechts heel lichte deubiquitinase activiteit heeft. ISG15 is een van de interferongeïnduceerde genen en lijkt qua structuur erg op een di-ubiquitine keten. ISG15 kan, net als ubiquitine, covalent gebonden worden aan eiwitten, al is de rol hiervan nog onduidelijk. We verklaren de extreme specificiteit van leader voor ISG15 doormiddel van het ophelderen van de kristalstructuur van leader in complex met ISG15. Uit deze kristalstructuur blijkt dat ubiquitine een voor de interactie met leader belangrijke tryptofaan mist, welke wel aanwezig is in ISG15.

In hoofdstuk 4 onderzochten we vervolgens de rol van leader's deISGylase activiteit in het onderdrukken van type I-interferon. Het knippen van RLR-signaleringseiwitten door leader is ook gesuggereerd om bij te dragen aan het onderdrukken van de type I-interferon productie. In dit hoofdstuk hebben we eerst systematisch onderzocht welke eiwitten in de RLR-signaaltransductieroute geknipt worden door leader. Met een set van mutanten hebben we onderzocht welke proteolytische activiteit (het knippen van RLR-signaleringseiwitten of de deISGylase activiteit) bijdraagt aan het onderdrukken van de expressie van IFN- $\alpha / \beta$. De deISGylase activiteit van leader bleek niet belangrijk te zijn, terwijl onderdrukking van type I-interferon expressie wel correleert met het knippen van RLR-signaleringseiwitten door leader.

In hoofdstuk 5 onderzochten we de humane enterovirussen. Enterovirussen hebben twee proteasen, $2 \mathrm{~A}^{\text {pro }}$ en $3 \mathrm{C}^{\text {pro }}$. Voor beide proteasen is gesuggereerd dat ze belangrijk zijn in het onderdrukken van antivirale responsen. Hierdoor bleef het exacte mechanisme waarmee enterovirussen deze antivirale responsen onderdrukken, en het verantwoordelijke virale eiwit, onduidelijk. In dit hoofdstuk hebben we de $2 \mathrm{~A}^{\text {pro }}$ en $3 \mathrm{C}^{\text {pro }}$ van representatieve enterovirussen van de verschillende humane enterovirus genera (EV-A t/m EV-D) vergeleken. Voor elk van deze proteasen hebben we onderzocht of deze de expressie van type I-interferon en de vorming van stress granules kan onderdrukken. Uit deze omvangrijke vergelijking blijkt dat voor alle geteste enterovirussen het $2 \mathrm{~A}^{\text {pro }}$ de belangrijkste antagonist is van beide antivirale responsen. Van de stress granules is gesuggereerd dat deze een rol spelen als signaleringsplatform voor verdere antivirale responsen, bijvoorbeeld voor de inductie van type I-interferon expressie. Omdat $2 \mathrm{~A}^{\text {pro }}$ een negatief effect heeft op zowel de expressie van IFN- $\alpha / \beta$ als op de vorming van stress granules, hebben we het gesuggereerde verband tussen deze twee antivirale responsen in meer detail onderzocht. Onze data suggereren dat stress granules een rol spelen in de RLR-signaaltransductieroute, maar dat ze niet essentieel zijn voor IFN- $\alpha / \beta$ expressie. Bovendien laten wij zien dat de onderdrukking van type I-interferon expressie door enterovirus $2 \mathrm{~A}^{\text {pro }}$ een veelvoud is van de relatief kleine bijdrage van SGs aan deze signaleringsroute, wat suggereert dat $2 \mathrm{~A}^{\text {pro }}$ een additioneel mechanisme heeft om de RLR-signaaltransductieroute direct te kunnen onderdrukken.

In hoofdstuk 6 onderzochten we de kobuvirussen. Het humane kobuvirus, aichivirus, werd in de jaren ' 90 in Japan ontdekt, en er is nog weinig bekend over het onderdrukken van antivirale responsen door dit virus. Wij ontdekten dat de leader van dit virus, ditmaal geen protease, een uniek mechanisme heeft om de cellulaire stressrespons te onderdrukken. Zoals eerder beschreven kunnen virussen de cellulaire stressrespons onderdrukken op grofweg twee manieren; het tegengaan of omzeilen van de signaaltransductie route waardoor er geen sprake is van p-eIF2, of het tegengaan van de vorming van stress granules. In dit hoofdstuk beschrijven we dat het leader eiwit van aichivirus een directe interactie aangaat met het eIF2B complex, waardoor translatie kan plaatsvinden in de aanwezigheid van gefosforyleerd eIF2. In een niet-geïnfecteerde cel, gaat eIF2 een interactie aan met het eIF2B complex om geladen te worden met een nieuw GTP en zo gebruikt te kunnen worden in een volgende ronde van translatie. Ten tijde van stress kan de cel eIF2 fosforyleren. Het gevormde p-eIF2 heeft een inhiberend effect op het eIF2B complex, waardoor het niet-gefosforyleerde eIF2 niet langer wordt gerecycled en de translatie tot stilstand komt. In aanwezigheid van aichivirus leader zien wij dat translatie ongehinderd is, ondanks de aanwezigheid van p-eIF2. Eenzelfde mechanisme is slechts een keer eerder geïdentificeerd, het accessory protein 10 van beluga whale coronavirus bindt ook aan eIF2B om translatie in aanwezigheid van p-eIF2 te faciliteren. Vervolgens hebben we onderzocht of dit mechanisme geconserveerd is in de andere kobuvirussen. Dit bleek enkel het geval voor het leader eiwit van het nauwverwante muizenkobuvirus, wat suggereert dat de onderdrukking van de cellulaire stressrespons door leader een recente evolutionaire ontwikkeling is, en leader hoogstwaarschijnlijk een andere primaire functie heeft. 


\section{Dankwoord}

Welcome dear readers, you have made it to the most sought-after section of any thesis, the acknowledgments. In your hands is the result of 5 years of hard work. I want to invite you to also have a quick look at the other chapters of this thesis before putting it on your bookshelf and returning to the lab. I would like to thank everyone who contributed to my scientific development, in any way, shape or form. Completing a PhD project is a truly lifechanging event.

Als eerste wil ik mijn promotor bedanken. Frank, ik ben onder de indruk dat je mijn chaotische gedachtengang kon volgen en mij vervolgens hebt geleerd om mijn plannen en ideeën te stroomlijnen. Ik denk dat we beiden tevreden zijn met het proefschrift, maar het heeft wat bloed, zweet en tranen gekost. Ik heb jouw eindeloze support en feedback erg gewaardeerd. In jouw overvolle agenda was altijd wel een gaatje te vinden voor overleg, al was dat soms erg vroeg in de ochtend! Bedankt voor jouw begeleiding in mijn wetenschappelijke ontwikkeling.

Ik was in de luxepositie dat ik kon rekenen op de steun van twee copromotoren. Martijn, bedankt voor al jouw hulp tijdens mijn promotietraject. In de beginfase heb ik ontzettend veel gehad aan jouw hulp en supervisie, zowel bij het plannen als het uitvoeren van experimenten. Ook nadat je de groep van Frank had verlaten was jij altijd beschikbaar voor vragen, super bedankt voor jouw voortdurende support. Jouw vertrek betekende voor mij een periode van persoonlijke groei. Soms moet je in het diepe worden gegooid! In deze periode kon ik gelukkig vertrouwen op de mening van mijn tweede copromotor. Raoul, jouw eindeloze stroom van kritische vragen en opmerkingen was soms wat frustrerend maar heeft wel mateloos bijgedragen aan mijn wetenschappelijke ontwikkeling. Daarnaast wil ik je ook bedanken voor de fascinerende koffiekamergesprekken. Ik heb me uitstekend vermaakt tijdens de spontane discussies over talloze onderwerpen, variërend van politiek tot de nationale obsessie met het weer

Dan kom ik uit bij mijn twee paranimfen, Huib en Judith. Bedankt dat jullie mij op deze bijzondere dag willen bijstaan, zoals jullie dat de afgelopen jaren al hebben gedaan. Huib, het vertrek van Martijn betekende dat wij intensiever gingen samenwerken. We hadden talloze spontane brainstormsessies in labs, op de gang, of bij de koffieautomaat, die hebben bijgedragen aan de succesvolle afronding van mijn projecten. Ook bedankt voor het delen van cellen, FACS - of microscopietijd, en gelletjes. Ik moet je natuurlijk ook bedanken voor het belangrijkste advies ooit: "houd je nooit aan een protocol". Judith, je liep stage bij viro toen ik begon aan mijn PhD en ondertussen ben je alweer terug voor een post-doc! Thnx voor jouw vriendschap al deze jaren (en in de toekomst) en voor Gerrit ;).
A special thank you goes to all my collaborators: Gisselle Medina, Teresa de los Santos, Kirby Swatek, David Komander, Karin Olek, Tim Skern, Piero Giansanti and Albert Heck. Thank you for your valuable input on my projects both through experiments and inspiring discussions.

Lisa, FOCUS! And if everything else fails, add some unicorn sparkle. Thank you for the countless coffee breaks, oxygen breaks, 4-degrees room breaks and of course the occasional after-work beer. Amazing that we actually got work done! Maryam, ik kan me jouw eerste dag in het lab nog steeds herinneren... Bedankt voor al je inzet voor de innate immunity groep en bedankt voor de dagelijkse dosis zonneschijn die jij met je meebrengt. Heyrhyoung, I'll promise to never say 'I'm drinking babies' in Korean. Yifei, I'm going to keep you to your promise to show me China. Chiara, it was a pleasure to hand over the ongoing innate immunity research to you. I'm sure you will make a success of your PhD project!

During the years, I had many roomies. Wentao and Meiling, thank you for your warm welcome into the office and for always helping out, answering questions and for making me try the interesting Chinese food items. Floor, het was supergezellig om samen onze eerste dag bij viro te hebben! Daarnaast wil ik je bedanken voor de Hek293T's, de midipreps, maar vooral voor jouw steun en vriendschap. Cristina, Lucian and Jeroen, thank you for welcoming me into the crazy world of office 504. A daily dose of minions, Romanian swearing and regular fruit breaks is all you need. Jeroen, bedankt voor jouw advies als een van mijn experimenten in de soep liep, het zijn van een luisterend oor, mij voorzien van tenminste een woordgrap per dag, en jouw bijdrage aan de succesvolle afronding van mijn proefschrift. Fiona, you replaced Cristina but you did so much more than replace someone. Thank you for sharing your Mass Spec knowledge, but more importantly thank you for your friendship. Dan, you were the first of the new generation of 504-ers and I'm sure you will take good care of the office. It was fun to introduce you and the cat calendar to the lab, and I'm convinced that you'll be successful in your $2 \mathrm{C}$ project. Mirthe and Irina, I'm sure you will have a great time in the lab. Please take care of the Yucca?

Nancy en Arno, jullie zijn de stevige fundering van de virologieafdeling. Bedankt voor jullie harde werk, het is niet onopgemerkt gebleven. Natuurlijk ook bedankt voor de lol tijdens woordgraptogram-maandag.

I was fortunate to supervise several students: Walae, Jesus and José thank you for your hard work. Tim, je hebt een geweldige MSc scriptie geschreven waarin ik meermaals iets heb opgezocht tijdens het schrijven van dit proefschrift. 
Thanks to all my amazing colleagues over the years: Ivy, Jim, Ieva, Hilde, Rachel, Jojanneke, Hendrik, Ruben, Mark, Erik, Herman, Peter, Clasien, Anja, Berend-Jan, Xander, Huihui, Hongbo, Wenjuan, Shan, Joline, Vera, Brenda, Esther, Malte, Erhard, Susanne, Kyra and Sandra.

Last but not least, wil ik mijn vrienden en familie bedanken voor hun steun. Papa, vanaf nu zal ik weer wat meer tijd hebben en vaker langskomen! Gerrit, sorry dat ik een grumpy old lady was tijdens het schrijven van mijn proefschrift. Ik beloof je dat ik nu weer 'normaal' word.

\section{About the author}

Linda Jessica Visser was born on May $26^{\text {th }} 1991$ in Apeldoorn, the Netherlands. In 2009 she graduated from bilingual pre-university education (T-VWO) at the Koninklijke Scholengemeenschap in Apeldoorn. That same year, she started a bachelor degree in Biomedical Sciences at Utrecht University, obtaining her degree in 2012. Linda continued her education via the master program Infection \& Immunity at Utrecht University. As a part of this program she conducted an internship at the UMC Utrecht medical center under the supervision of dr. M.L. van de Weijer, dr. R.J. Lebbink and prof. E.J.H.J. Wiertz. Here, she studied how the CMV US2 protein interferes with antigen presentation. A second internship under supervision of prof. G. van den Bogaart was conducted at the Radboud Institute for Molecular Life Sciences. Here, Linda investigated the molecular details of the immunological synapse in dendritic cells. During this master program, Linda got the opportunity to apply for $\mathrm{PhD}$ project funding via the NWO graduate program. The research proposal written by her, dr. M.A. Langereis and prof. F.J.M. van Kuppeveld acquired funding, and in 2014 Linda started as a PhD candidate at the Faculty of Veterinary Medicine at Utrecht University. She studied the strategies employed by picornavirus security proteins to evade or counteract innate antiviral responses. The results on this research are described in this thesis. 


\section{List of publications}

\section{This thesis}

Visser LJ, Aloise C, Swatek KN, Medina GN, Olek KM, Rabouw HH, de Groot RJ, Langereis MA, de Los Santos T, Komander D, Skern T, van Kuppeveld FJM. 2020. Dissecting distinct proteolytic activities of FMDV Lpro implicates cleavage and degradation of RLR signaling proteins, not its deISGylase/DUB activity, in type I interferon suppression. PLoS Pathog.

Rabouw HH, Visser LJ, Passchier TC, Langereis MA, Liu F, Giansanti P, van Vliet AL, Dekker JG, van der Grein SG, Saucedo JG, Anand AA, Trellet ME, Bonvin AMJJ, Walter P, Heck AJR, de Groot RJ, van Kuppeveld FJM. 2020. Inhibition of the integrated-stress-response by viral proteins that block p-eIF2•IF2B association. Nat Microbiol.

Visser LJ, Langereis MA, Rabouw HH, Wahedi M, Muntjewerff EM, de Groot RJ, van Kuppeveld FJM. 2019. Essential Role of Enterovirus 2A Protease in Counteracting Stress Granule Formation and the Induction of Type I Interferon. J Virol.

Visser LJ, Medina GN, Rabouw HH, de Groot RJ, Langereis MA, de Los Santos T, van Kuppeveld FJM. 2019. Foot-and-Mouth Disease Virus Leader Protease Cleaves G3BP1 and G3BP2 and Inhibits Stress Granule Formation. J Virol.

Swatek KN, Aumayr M*, Pruneda JN*, Visser LJ*, Berryman S, Kueck AF, Geurink PP, Ovaa H, van Kuppeveld FJM, Tuthill TJ, Skern T, Komander D. 2018. Irreversible inactivation of ISG15 by a viral leader protease enables alternative infection detection strategies. Proc Natl Acad Sci U S A. "These authors contributed equally.

\section{Additional publications}

Rabouw HH, Langereis MA, Anand AA, Visser LJ, De Groot RJ, Walter P, Van Kuppeveld FJM. 2019. Small molecule ISRIB suppresses the integrated stress response within a defined window of activation. Proc Natl Acad Sci U S A.

Van De Weijer ML, Van Muijlwijk GH, Visser LJ, Costa AI, Wiertz EJHJ, Lebbink RJ. 2016. The E3 ubiquitin ligase TMEM129 is a tri-spanning transmembrane protein. Viruses.

Verboogen DRJ, Dingjan I, Revelo NH, Visser LJ, Ter Beest M, Van Den Bogaart G. 2016. The dendritic cell side of the immunological synapse. Biomol Concepts.

Dingjan I, Verboogen DRJ, Paardekooper LM, Revelo NH, Sittig SP, Visser LJ, Von Mollard GF, Henriet SSV, Figdor CG, Ter Beest M, Van Den Bogaart G. 2016. Lipid peroxidation causes endosomal antigen release for cross-presentation. Sci Rep.

Langereis MA, Rabouw HH, Holwerda M, Visser LJ, van Kuppeveld FJM. 2015. Knockout of cGAS and STING Rescues Virus Infection of Plasmid DNA-Transfected Cells. J Virol. 
Chapter 8 - Addendum 